\title{
Ulrich Siegrist
}

\section{Die Rolle des Felt Sense im Coaching}

Eine explorative Studie 
Ulrich Siegrist

\section{Die Rolle des Felt Sense im Coaching}

Eine explorative Studie 
Die vorliegende Arbeit wurde vom Fachbereich Humanwissenschaften der Universität Kassel als Dissertation zur Erlangung des akademischen Grades eines Doktors der Philosophie (Dr. phil.) angenommen.

\author{
Gutachter: Prof. Dr. Heidi Möller \\ Prof. Dr. Silja Kotte
}

Tag der mündlichen Prüfung: 28. Oktober 2020

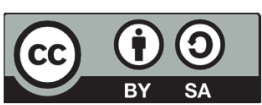

Diese Veröffentlichung - ausgenommen Zitate und anderweitig gekennzeichnete Teile - ist unter der Creative-Commons-Lizenz Namensnennung - Weitergabe unter gleichen Bedingungen International (CC BY-SA 4.0: https://creativecommons.org/licenses/by-sa/4.0/deed.de) lizenziert.

Bibliografische Information der Deutschen Nationalbibliothek

Die Deutsche Nationalbibliothek verzeichnet diese Publikation in der Deutschen Nationalbibliografie; detaillierte bibliografische Daten sind im Internet über http://dnb.dnb.de abrufbar.

Zugl.: Kassel, Univ., Diss. 2020

ISBN 978-3-7376-0918-0

DOI: https://doi.org/doi:10.17170/kobra-202011182205

(C) 2020, kassel university press, Kassel

https://kup.uni-kassel.de

Printed in Germany 


\section{Dank}

Auch wenn ich diese Dissertation eigenständig verfasst habe, ist sie doch ein Werk, das ich alleine nicht hätte bewältigen können. Bei aller Anstrengung, die damit verbunden war, empfinde ich es am Ende als Privileg, dass ich diese Arbeit anfertigen konnte.

Mein erster Dank geht an meine Betreuerin Prof. Dr. Heidi Möller, die nicht nur bereit, sondern auch interessiert war, mich als externen Doktoranden zu betreuen. Sehr inspirierend waren für mich zweimal jährlich die von Prof. Dr. Möller geleiteten Kolloquien, die mich eingebunden haben in die Forschungscommunity am Institut für Psychologie der Universität Kassel. Die vielfältigen Diskussionen dort haben mich akademisch weit über mein eigenes Forschungsthema hinaus geprägt. Ganz herzlichen Dank auch den Teilnehmenden, die mich immer wieder mit ihrem konstruktiven und kollegialen Feedback unterstützt haben. Dass ich als externer Doktorand an der Kasseler Coachingstudie teilhaben konnte, dort an Tagungen teilnehmen und die Daten der Studie für mein Forschungsprojekt nutzen konnte, war für mich eine wichtige Ressource, und ich danke Prof. Dr. Silja Kotte für das zusätzliche Engagement, das sie in diesem Zusammenhang für mich investierte.

Eine kritische Phase ergab sich für mich, als sich herausstellte, dass ich trotz aller Bemühungen für mein ursprünglich geplantes Forschungsvorhaben keine adäquate Datenbasis erhalten würde, so dass ich mein Forschungsprojekt neu konzipieren musste. Hier erlebte ich Prof. Dr. Möller besonders unterstützend und lösungsorientiert, und ich bin froh um die Wende, die mein Projekt dadurch nahm.

Zahlreiche Kolleginnen und Kollegen haben mich während meines Forschungsprozesses immer wieder interessiert begleitet. Stellvertretend möchte ich Prof. Dr. Renate Schwarz und Clemens Janosch nennen. Danke, dass ich mit euch auch inhaltlich im Austausch über meine Arbeit sein konnte.

Ein herzlicher Dank geht an die Coachs und Coachees, die letzten Endes Audioaufzeichnungen für die Studie zur Verfügung gestellt haben. Ich weiß diese Offenheit zu schätzen - ohne sie wäre die vorliegende Arbeit nicht möglich gewesen.

Ganz besonders erwähnen möchte ich meinen Lieblingsmenschen Simone Siegrist. Nicht nur, dass du mir in der zurückliegenden Zeit den Rücken freigehalten hast, sondern mehr noch, dass du in die oft langen Stunden des Forschens und Schreibens Farbe und Abwechslung gebracht und es mir leicht gemacht hast, die Balance im Leben zu halten. Du hast in allen Phasen an mich geglaubt, das hat gut getan.

Immer wieder habe ich in der Zeit des Schreibens auch meine Eltern Käthe und Werner Siegrist vor Augen gehabt, besonders meinen Vater, der mir ein Vorbild geworden ist, im Leben nicht stehen zu bleiben, immer wieder neue Herausforderungen zu suchen und sich 
dabei Zufriedenheit und ein tiefes Vertrauen in sein gottgeschenktes Leben zu bewahren. Ohne diese Grundlage hätte ich diese Arbeit vermutlich nicht umsetzen können. Danke! 


\section{Abstract}

In Focusing and the experiential concept of change, the construct of Felt Sense plays a specific role. This seems to be relevant for coaching, considering neuroscientific findings and the demand to include affective or emotional and body-related aspects in coaching.

The present study investigates intrapersonal moments of change in the context of coaching and connects them with experiential concepts. It uses the method of qualitative task analysis, in which a theoretical model of change is compared with empirical findings in an abductive procedure using three ideal-typical and three contrasting coaching episodes.

An empirical-theoretical model will be developed that maps the intra-individual process of change and allows conclusions to be drawn about experiential processes in coaching. Implications for the promotion of intrapersonal change processes will be derived from this model. Trilemma-situations in the sense of an incongruence or tension between situational reference and contradictory parts of affective-cognitive perception respectively experiencing are worked out as a central starting point for moments of change. 


\section{Zusammenfassung}

Im experienziellen Veränderungskonzept des Focusing spielt das Konstrukt des Felt Sense eine besondere Rolle. Für das Coaching scheint das insofern relevant, als dass sich dort vor dem Hintergrund neurowissenschaftlicher Erkenntnisse zunehmend die Frage nach dem Einbezug affektiver bzw. emotionsbezogener und körperbezogener Aspekte stellt.

Die vorliegende Studie untersucht intrapersonale Veränderungsmomente im Rahmen von Coaching und bringt sie in Verbindung mit experienziellen Konzepten. Sie bedient sich der Methode der qualitativen Task Analyse, bei der in einem abduktiven Verfahren anhand von drei idealtypischen und drei kontrastierenden Coachingepisoden ein theoretisches Veränderungsmodell mit empirischen Befunden abgeglichen wird.

Es wird ein empirisch-theoretisches Modell entwickelt, das den intraindividuellen Veränderungsprozess abbildet und Rückschlüsse auf experienzielle Prozesse im Coaching zulässt. Daraus werden Implikationen zur Förderung intrapersonaler Veränderungsprozesse abgeleitet. Als zentraler Ausgangspunkt für Veränderungsmomente werden Trilemma-Situationen im Sinn einer Inkongruenz oder Spannung zwischen Situationsbezug und kontradiktorischen Anteilen der affektiv-kognitiven Wahrnehmung bzw. des Selbsterlebens herausgearbeitet. 


\section{Inhalt}

$1 \quad$ Einführung ..........................................................................................12

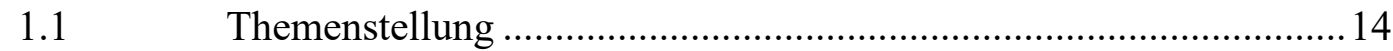

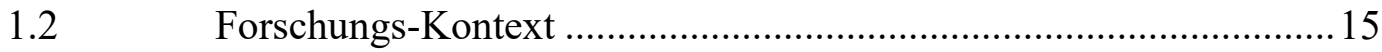

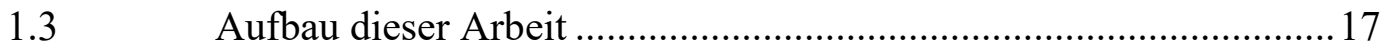

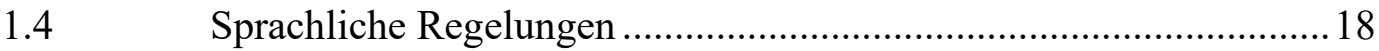

$1.5 \quad$ Umgang mit Anlagen .................................................................. 18

$2 \quad$ Coaching................................................................................................. 19

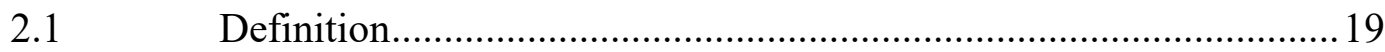

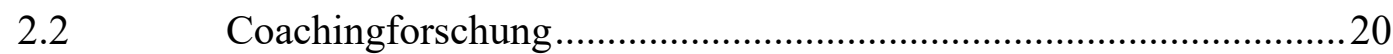

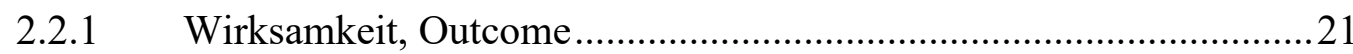

2.2.2 Wirkfaktoren, Prozess ....................................................................22

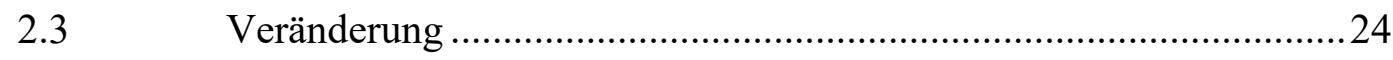

$3 \quad$ Experiencing ………………..................................................................29

3.1 Psychologische und philosophische Grundlagen ……………….......30

3.2 Gendlins Theorie des Experiencing................................................... 34

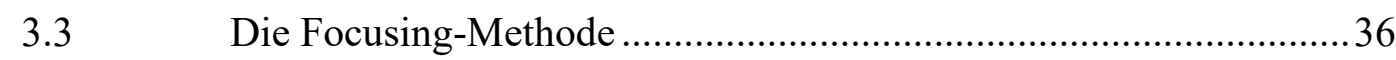

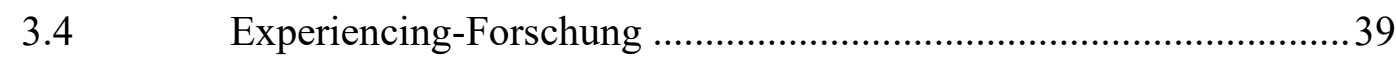

3.5 Kritische Würdigung des Experiencing-Konzepts ...............................4

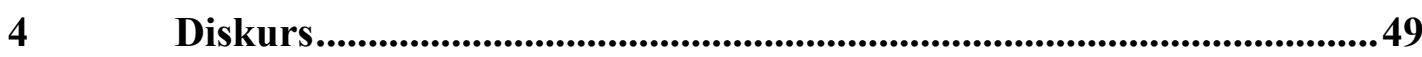

$4.1 \quad$ Personzentrierung und Coaching ...................................................... 49

4.2 Neurowissenschaften und Körperzentrierung ..................................52

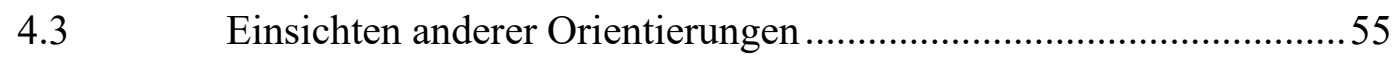

4.3.1 Körperorientierte Verfahren ............................................................ 55

4.3.2 Achtsamkeitsbasierte Verfahren.......................................................57

4.3.3 Psychoanalytische Konzepte …………………….........................59

4.3.4 Hypnosystemische Beratung ……………………………………...... 60

4.3.5 Prozess-Experienzieller Emotionsfokussierter Ansatz..........................62

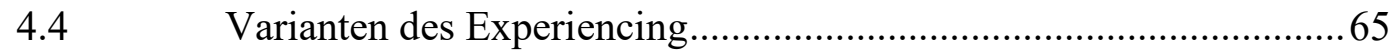

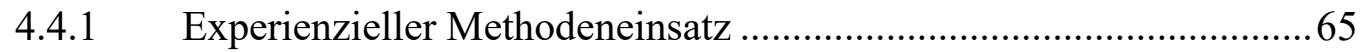

4.4.2 Thinking at the Edge und erlebensorientiertes Concept Coaching ....67

$4.5 \quad$ Zwischenfazit ............................................................................ 70 


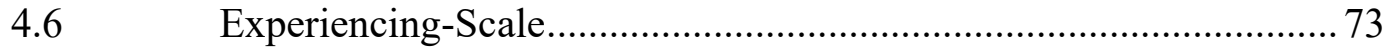

5 Forschungsprojekt: Methodische Grundlagen und Ziele..................... 79

5.1 Einordnung und Ziele der Studie ............................................... 79

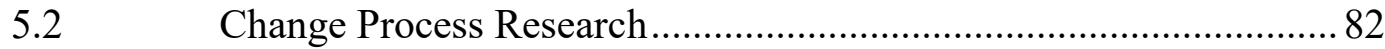

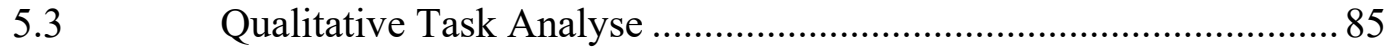

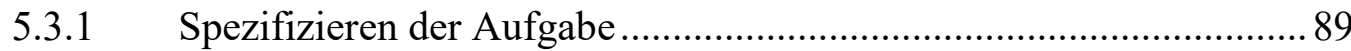

5.3.2 Erklären der kognitiven Karte ...................................................... 90

5.3.3 Spezifizieren der Rahmenbedingungen........................................... 91

5.3.4 Bilden eines theoretischen Aufgabenmodells ................................. 92

5.3.5 Durchführen der empirischen Analyse............................................. 92

5.3.6 Erstellen eines theoretisch-empirischen Modells ............................. 95

5.3.7 Theoriebasiertes Erklären des modizifierten Modells........................ 95

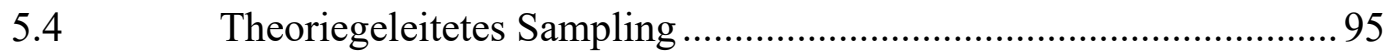

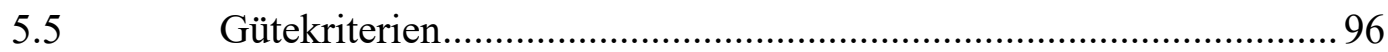

6

Durchführung der Studie ................................................................99

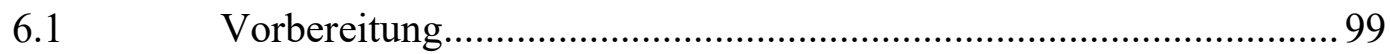

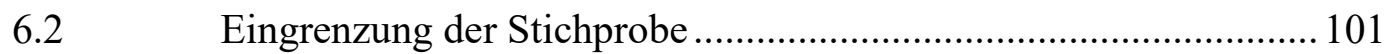

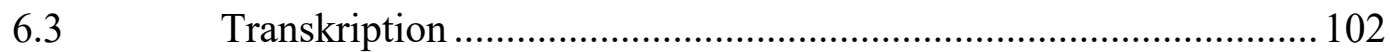

6.4 Aufgabe: Umgehen mit dem Unklaren ........................................ 103

Kognitive Karte: bottom up........................................................ 109

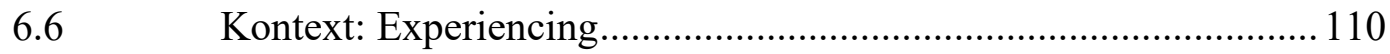

6.7 Theoretisches Modell: vom Unklaren zum Felt Shift .................... 112

6.8 Empirische Task Analyse ........................................................ 113

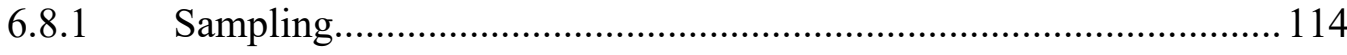

6.8.2 Codierung der Episoden ............................................................... 117

6.8.3 Empirische Modellbildung .......................................................... 120

6.8.4 Rekonstruktion des empirisch-theoretischen Modells .................... 135

6.8.5 Theoretische Rückbindung: Der Felt Sense im Coaching ............... 156

$7 \quad$ Implikationen für das Coaching ........................................................... 161

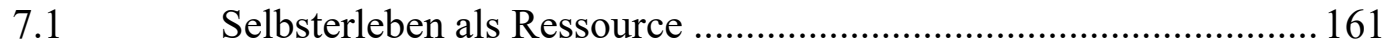

7.2 Der Felt Sense als gefühlte Bedeutung ......................................... 164

7.3 Vom Felt Sense zum Felt Shift ................................................. 165

7.4 Trilemma-Situation als Ausgangspunkt ....................................... 168

7.5 Indikationen und Kontraindikationen........................................... 170

7.6 Vom Trilemma zur Dialektik innerer Erlebensanteile .................... 172

7.7 Ausstiegsmomente aus dem Experiencing ................................... 173 
7.8 Coachverhalten und Methodengebrauch .................................... 174

7.8.1 Personzentrierte Beziehungsvariablen........................................... 175

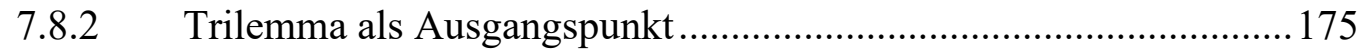

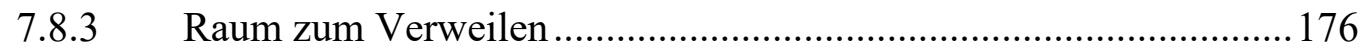

7.8.4 Prozessorientierung und Zielorientierung ...................................... 178

7.8.5 Umgang mit Zweifeln und Unsicherheit ...................................... 178

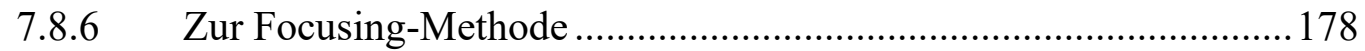

7.8.7 Methodischer Impuls: Trilemma-Übung...................................... 179

$8 \quad$ Methodische Reflexion ..................................................................183

Indikationen zur Methodenwahl ................................................... 183

8.2 Theorie- und Praxisorientierung in der Task Analyse..................... 185

8.3 Übertragbarkeit der Studie ......................................................... 186

8.4 Vertrauenswürdigkeit und Zuverlässigkeit der Studie .................... 187

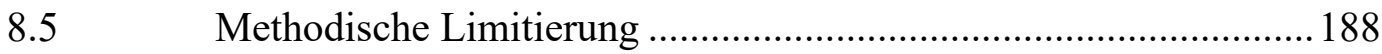

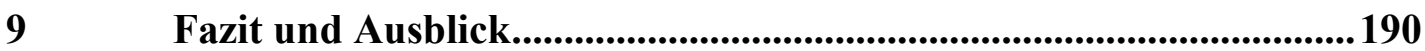

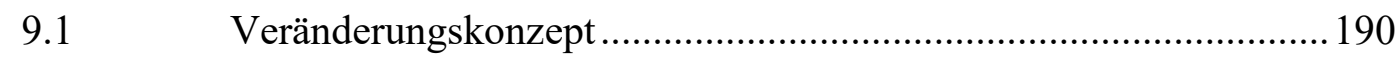

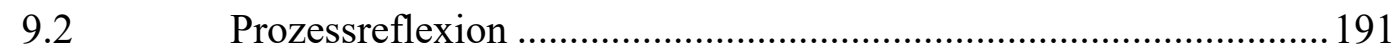

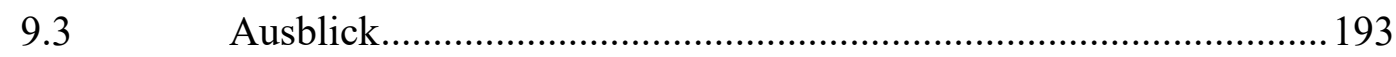

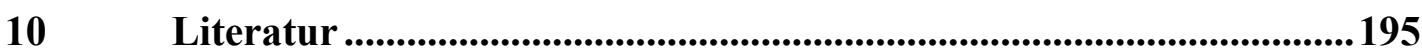

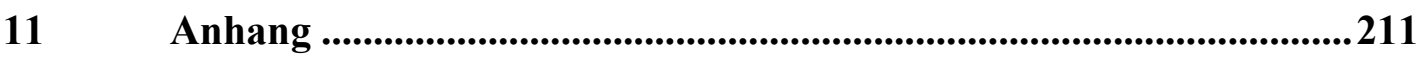

11.1 Verzeichnis der digitalen Anlagen ............................................211

11.1.1 Anlagen zur Task-Analyse ........................................................211

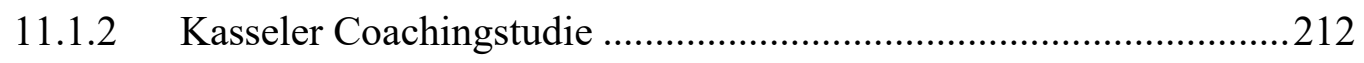

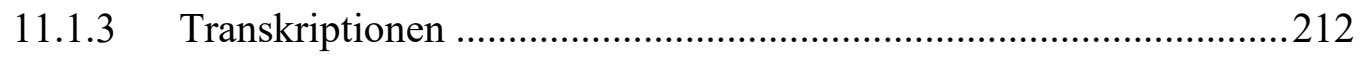




\section{Abbildungen}

Abbildung 1: Theoretisches Aufgabenmodell im Veränderungsprozess. 113

Abbildung 2: Empirisches Modell zur ersten idealtypischen Episode. 122

Abbildung 3: Empirisches Modell zur zweiten idealtypischen Episode 125

Abbildung 4: Empirisches Modell zur dritten idealtypischen Episode 128

Abbildung 5: Empirisches Modell zur ersten kontrastierenden Episode 131

Abbildung 6: Empirisches Modell zur zweiten kontrastierenden Episode 133

Abbildung 7: Empirisches Modell zur dritten kontrastierenden Episode 135

Abbildung 8: Gemeinsames empirisches Modell 144

Abbildung 9: Experiencing-Stufen der untersuchten Episoden im Vergleich 148

Abbildung 10: Synthetisiertes theoretisch-empirisches Modell 155

Abbildung 11: Trilemma 168

Abbildung 12: Trilemma-Beispiel aus Episode 2017_1805_01\#1 169

Abbildung 13: Trilemma-Beispiel aus Episode 2016_1119a_01\#8 169

Abbildung 14: Trilemma-Beispiel aus Episode 2017_2119_03\#3 169 


\section{Tabellen}

Tabelle 1: Gegenüberstellung: Übersetzungsprozess affektiver Schemata und Vorgehen im Focusing

Tabelle 2: Kommunikation: In-Beziehung-Sein versus In-Funktion sein (Hofmann, 2016) 52

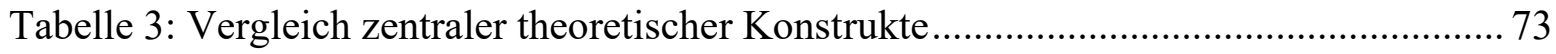

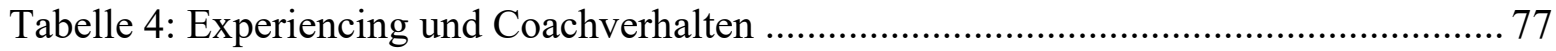

Tabelle 5: Codes auf Grundlage des theoretischen Modells ............................................. 118

Tabelle 6: Übereinstimmende Higher-Level-Kategorien der Makrokategorie Selbsterleben in

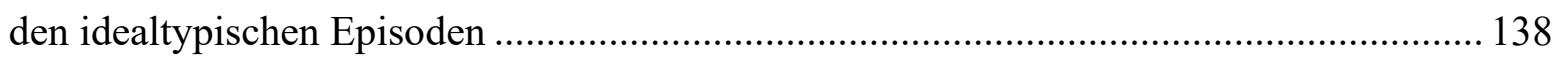

Tabelle 7: Übereinstimmende Higher-Level-Kategorien der Makrokategorie Kontextbezug in den idealtypischen Episoden

Tabelle 8: Übereinstimmende Higher-Level-Kategorien der Makrokategorie Selbsterleben in den kontrastierenden Episoden. 140

Tabelle 9: Übereinstimmende Higher-Level-Kategorien der Makrokategorie Kontextbezug in den kontrastierenden Episoden. 141

Tabelle 10: Übersicht der Kernmerkmale der idealtypischen Episoden 142

Tabelle 11: Übersicht der übereinstimmenden Merkmale der kontrastierenden Episoden. 142 Tabelle 12: Kernmerkmale idealtypischer Prozesse und Codierungen der kontrastierenden Episoden im Vergleich 146

Tabelle 13: Kategorien des theoretischen Modells und ihre Vorkommen in den idealtypischen Episoden 151

Tabelle 14: Dialektik innerer Erlebensanteile (Beispiele) 173 


\section{Einführung}

„Persönliche Probleme und Schwierigkeiten im Leben sind niemals nur kognitiver Natur, niemals nur eine Frage von Interpretation und Verstehen. Es gibt immer eine affektive, emotionale, gefühlte, konkrete, erlebte Schwierigkeit. Die Gedanken und Interpretationen einer Person entstehen aus und sind beeinflusst von ihrer gefühlsgeprägten Art, in der jeweiligen Situation zu leben. (...) Was wir fühlen, ist kein inneres Objekt (ein ,affektiver“ Zustand, der nur in uns ist), sondern der Felt Sense zu einer umfassenden Situation - wie wir in dieser Situation sind, was wir hervorbringen, was wir wahrnehmen und womit wir uns konfrontiert fühlen. (...) Dieser Felt Sense bezieht sich auch darauf, wie wir die Situation interpretiert und aufgefasst haben. Deshalb ist ein solcher Felt Sense nicht nur etwas Gefühltes, sondern auch etwas Kognitives. Vielleicht verwirrt uns, was er mit sich bringt, aber immer sind Aspekte der Interpretation, d.h. Denken, Lernen, Wahrnehmen und Verstehen in ihm impliziert.“(Gendlin, 2002, S. 1)

Dass persönliches Problemerleben und persönliche Lösungs- und Veränderungsprozesse nicht nur kognitiver Natur sind, sondern auch eine emotionale oder affektive, oft körperlich spürbare Komponente beinhalten, ist weder in der Philosophie noch in der Psychologie eine neue Erkenntnis. So wies schon der altjüdische Philosoph David (ca. 1000 v. Chr.) auf einen Zusammenhang zwischen körperlichem und affektivem Erleben hin und regte in einer schwierigen Situation an zu einem inneren Dialog zwischen kognitiven und körperlichen bzw. affektiven Erlebensinhalten (Die Bibel, 1999, Psalm 32 \& 42). Auch Aristoteles (384 - 322 v. Chr.) beschäftigte sich mit den Zusammenhängen zwischen seelischen und körperlichen Zuständen und kam zu der Erkenntnis, dass die Seele mit dem Körper verbunden sei (Perler, 1996, S. 346).

Das neuzeitliche Menschenbild war lange Zeit wesentlich geprägt von René Descartes (15961650), der als Begründer des modernen frühneuzeitlichen Rationalismus die Auffassung von Geist und Materie als voneinander unabhängiger Substanzen vertrat (vgl. Damasio, 1994). Mit dem Aufkommen des Humanismus und des Existenzialismus und der darauf aufbauenden Humanistischen Psychologie im 20. Jahrhundert wurde das descartessche Verständnis von Leib und Seele als getrennte Entitäten allerdings zunehmend abgelöst durch eine den GesamtOrganismus des Menschen als Einheit betrachtende Sichtweise (Ryba, 2019, S. 314). Hervorzuheben ist in diesem Zusammenhang Carl Rogers (1902-1987), der in seiner klientenzentrierten Theorie dem ,inneren Erleben“ des gesamten Organismus eine besondere Bedeutung gab und dieses ins Verhältnis zum äußeren Erleben und Verhalten des Menschen setzte (Rogers, 2000, S. 138). 
Einen weiteren Zugang zu den Zusammenhängen zwischen Körper und Psyche bietet das von Petzold (2006) postulierte Konzept der Leiblichkeit, das nicht nur in der Leib- und Bewegungstherapie Niederschlag findet, sondern insbesondere auch in den Formaten Supervision und Coaching, beispielsweise in Form des „,in-den-Körper-Spürens“ (Schwarz, 2017). Der Mensch wird dabei betrachtet als ein materiell-transmaterielles Leib-Subjekt, das sich als Körper-Seele-Geist-Einheit entwickelt und sich hin zu einer schöpferischen und souveränen Persönlichkeit zu entfalten sucht (ebd., S. 69).

In jüngerer Zeit erhält die Wechselwirkung zwischen Körper und Psyche neue Aufmerksamkeit vor dem Hintergrund der Hirnforschung und daraus resultierender neurowissenschaftlicher Erkenntnisse (Storch, Cantieni, Hüther \& Tschacher, 2006; Koch, 2011; Gugutzer, 2012; Roth \& Ryba, 2016). Hier bestätigt sich mit dem Entdecken der limbischen Ebenen in Unterscheidung zu einer kognitiv-sprachlichen Ebene die Erfahrung, dass die kognitiv-sprachliche Ebene zwar stark von den limbischen Ebenen, in denen auch emotionale Prozesse angesiedelt sind, beeinflusst werden kann, dass es umgekehrt aber schwierig bis unmöglich ist, starke emotionale Zustände rein gedanklich-rational zu kontrollieren (Roth \& Ryba, 2017).

Als Schüler und Kollege von Carl Rogers hat Eugene Gendlin (1926-2017) mit der Philosophy of the Implicit und dem Konzept des Experiencing die Bedeutung von körperlichen Empfindungen bei innerpsychischen Prozessen und bei der Lösung von Problemen spezifiziert und die klientenzentrierte Psychotherapie durch die Methode des Focusing zur experienziellen Psychotherapie weiterentwickelt (Gendlin, 1996), die durch das Einbeziehen des sogenannten Felt Sense auch für andere Therapieformen einen zusätzlichen Nutzen generieren kann (Sachse, Atrops, Wilke \& Maus, 1992, Vorwort; Gendlin, 1996, S. 276). Als Problemlöse-Methode, die Denken und Fühlen systematisch in Beziehung bringt und das Unklare einer Situation körperlich erlebbar werden lässt, hat Focusing inzwischen in verschiedenen Lebensbereichen Einzug gehalten (Gendlin, 1998, S. 135ff; Gendlin \& Wiltschko, 2016; Hofmann, 2016, S. 401ff).

War Focusing anfänglich noch als eine Art Pre-Therapy-Training gedacht, um Klienten zu ermöglichen, sich konstruktiv in den Therapieprozess einzubringen, wurde es für Gendlin bald ein Bedürfnis, dieses Wissen über seelische Weiterentwicklung nicht nur einem Fachpublikum, sondern allen Interessierten zur Verfügung zu stellen. Dies wurde für ihn auch ein politisches Anliegen. So wird heute ein Großteil seiner Studien, Artikel, Erfahrungen, wie auch die vieler seiner Weggefährten, auf der Internetseite des Focusing Institute New York (www.focusing.org) kostenlos zur Verfügung gestellt.

Im Blick auf Coaching und Supervision weisen einzelne Autoren ebenfalls auf den Nutzen von Focusing bzw. von experienzieller Kommunikation hin (Feuerstein \& Müller, 1998; Deloch \& Feuerstein, 2013; Wahl, 2018), allerdings ist das Konzept im Coaching noch wenig verbreitet. Dies könnte auch darauf zurückzuführen sein, dass Focusing als Methode eher die Anmutung eines therapeutischen oder selbsterfahrungsorientierten Settings hat, während Coachs sich im Kontext arbeitsweltlicher Beratung eher von Therapie und Selbsterfahrung 
abgrenzen.

Auch aus psychologischer und beratungswissenschaftlicher Sicht ist zu hinterfragen, welche Rolle Experiencing und der Felt Sense im Coaching tatsächlich einnehmen können oder sollen, und inwieweit die im Rahmen der Psychotherapieforschung gewonnenen Erkenntnisse zum Focusing und Experiencing auch für die Beratungsdienstleistung Coaching gelten. Wenn Experiencing dort tatsächlich auch einen Beitrag zur Zielerreichung zu leisten vermag, könnten experienzielle Kommunikation und Focusing sich künftig zu einem wichtigen und das bisherige Portfolio ergänzenden Bestandteil des Coaching entwickeln.

\section{$1.1 \quad$ Themenstellung}

Die internationale Focusing-Practitioner-Community beschäftigte sich im Jahr 2019 unter anderem mit folgenden Fragen:

- „What does Focusing add to Coaching? And vice versa?

- What happens when we "cross" Focusing with Coaching?

- How do we bring Focusing into our coaching or coaching-like work?

- What challenges have we faced when using Focusing with our coaching or coaching-like clients?” (The International Focusing Institute, 2019)

Während in der Focusing-Community ein aktiver Einsatz focusingorientierter Methoden im Coaching zugrunde gelegt wird, stellt sich aus einer forschenden Perspektive heraus zunächst einmal die Frage, welche Rolle der Felt Sense bzw. das Experiencing generell im Coaching spielen oder spielen können und wie genau die entsprechenden Prozesse im Coaching sich darstellen. Ein besonderes Augenmerk gilt dabei intrapersonalen Klärungs- oder Veränderungsprozessen. Zum einen sind diese Prozesse immer wieder zentraler Bestandteil von Coaching (vgl. Greif, Möller \& Scholl, 2018; Hauke, 2011; West-Leuer, 2015), und zum anderen bezieht sich das Konzept des Felt Sense bzw. Experiencing auf genau dieses intrapersonale Veränderungsgeschehen (vgl. Sachse et al, 1992, Vorwort; Gendlin, 1996, S. 39).

Vor diesem Hintergrund soll in dieser Arbeit nicht primär die Methode des Focusing im Vordergrund stehen, sondern der intrapersonale Veränderungsprozess im Zusammenhang mit dem Felt Sense, soweit er sich aus einer beobachtenden Perspektive und unabhängig von der jeweiligen theoretischen Orientierung des Coachs beschreiben lässt. In einem seiner Grundlagenwerke, „Focusing-orientierte Psychotherapie“, führt Gendlin (1996, S. 271ff) aus, dass zum einen neben dem Focusing weitere Möglichkeiten existieren, um experienzielle Prozesse und eine Referenz auf den Felt Sense zu initiieren und zu begleiten, und dass zum anderen erlebensbezogenes Vorgehen auch schulenunabhängig bzw. schulenübergreifend stattfinden kann. So wäre es eine wenig hilfreiche Engführung, diese Studie nur auf Focusing oder auf ein bestimmtes schulenbezogenes Vorgehen zu begrenzen, vielmehr soll es schulenübergreifend um Felt-Sense-Phänomene und Facetten experienzieller Prozesse im Coaching gehen. Dabei gilt es, die entsprechenden Phänomene des Felt Sense und des Experiencing im Kontext 
von Veränderung zu erfassen, zu beschreiben und gegebenenfalls zu systematisieren, um daraus Aussagen über ihre Rolle im Veränderungsgeschehen ableiten zu können.

So sollen in dieser Studie Coachingprozesse im Blick auf Veränderung und Experiencing exploriert werden. Konkret soll dabei folgenden Fragen nachgegangen werden:

- Welche Rolle spielt der Felt Sense im Coaching?

- Was berichten Coaching-Klienten über ihre inneren Prozesse im Coaching?

- Welche für das Coaching spezifischen Besonderheiten einer auf Experiencing basierenden intrapersonalen Veränderung lassen sich beschreiben?

- Welche Erkenntnisse über das Veränderungsgeschehen lassen sich daraus ableiten? Lassen sich bestimmte Veränderungsmuster beschreiben?

Auf der Grundlage dieser Exploration sollen nach Möglichkeit Schlüsse zum experienziellen Vorgehen im Coaching gezogen werden. Ein besonderes Interesse gilt dabei der Frage nach Hinweisreizen oder Indikationen, wann experienzielles Vorgehen besonders zu empfehlen ist, und auch der Frage, wie die konkrete Umsetzung im Rahmen eines Coachingprozesses aussehen kann.

Um die Coachingprozesse angemessen explorieren zu können, werden zunächst eine Reihe von konzeptuellen und begrifflichen Klärungen notwendig sein, insbesondere eine Präzisierung des hier zugrundeliegenden Coachingverständnisses und des Konzepts des Experiencing und Felt Sense. Dazu wird auch gehören, den aktuellen Forschungsstand zu Veränderungsprozessen im Coaching und zum Experiencing und Felt Sense darzustellen.

Die Exploration der Coachingprozesse wird sich an den Empfehlungen zur Change Process Research (Elliot, 2010) und zur Task Analyse (Greenberg, 1984a; 1984b, 2007) orientieren. Dabei soll der Schwerpunkt weniger auf der allgemeinen Analyse des Therapie- bzw. Coachingprozesses liegen, sondern darauf, veränderungsrelevante Elemente zu identifizieren, sie zu beschreiben und zu erklären (vgl. Greenberg, 1986).

Gendlin (1986) forderte von der Forschung therapeutischen Geschehens, vor allem Subprozesse in den Blick zu nehmen, diese zu beschreiben und zu analysieren:

- Was in diesem Subprozess wäre auch für andere Prozesse wünschenswert?

- Was kann nur durch Experiencing und nicht durch einen anderen Prozess geleistet werden?

- Wie könnte ein universell einsetzbares und erstrebenswertes Vorgehen aussehen?

Diesem Desiderat soll hier Rechnung getragen werden.

\subsection{Forschungs-Kontext}

„Coaching wird in Organisationen zunehmend nachgefragt und eingesetzt. (...) Die bisherigen wissenschaftlichen Ergebnisse konnten insgesamt ein positives Fazit im Hinblick auf die Wirksamkeit von Coaching ziehen. Die Frage, wie und wodurch 
Coaching wirkt, ist hingegen noch wenig untersucht. Als Forschungsgruppe der Universität Kassel führen wir eine umfassend angelegte Prozess-Ergebnis-Studie durch, d.h. wir untersuchen Einflussfaktoren auf die Wirksamkeit von Coaching. Wir möchten zeigen, was im Coaching tatsächlich passiert: Dazu gehen wir über die Einschätzungen von Coach und Coachee hinaus und analysieren Audioaufzeichnungen von Coachingprozessen.“ (Kotte, Müller \& Hennemann, 2019)

Die hier vorgestellte Arbeit ist eingebettet in die Kasseler Coachingstudie (KCS), bei der an der Universität Kassel unter der Leitung von Prof. Dr. Heidi Möller, unterstützt vom Deutschen Bundesverband Coaching e.V. (DBVC), der Deutschen Gesellschaft für Supervision und Coaching e.V. (DGSv) und der Österreichischen Vereinigung für Supervision und Coaching (ÖVS) in den Jahren 2017 bis 2020 Coachingprozesse sowohl im Blick auf die Ergebnisse als auch im Blick auf das Geschehen im Coachingprozess selbst erhoben wurden. Ziel der Kasseler Coachingstudie ist, im Rahmen der Prozess-Outcome-Forschung weitere Erkenntnisse über Wirkfaktoren und Wirkmechanismen im Coaching zu erhalten und damit einen Beitrag zur wissenschaftlichen Fundierung und zur Weiterentwicklung des Beratungsformats Coaching zu leisten.

Um Veränderungen im Lauf der Coachingprozesse möglichst realitätsnah zu erfassen, wurde die Kasseler Coachingstudie als naturalistisches, prospektiv angelegtes Forschungsprojekt mit drei Erhebungswellen konzipiert. Das Design folgt damit dem in der Psychotherapieforschung gängigen Vorgehen zur Untersuchung der Beziehung zwischen Variablen einer Population unter Bedingungen der therapeutischen Praxis. Im Gegensatz zu experimentellen Designs kann in diesem Fall auf eine Kontrollgruppe verzichtet werden (Dempster, 2017, S. 31), was die Datenerhebung wesentlich erleichtert. Der Vorteil der naturalistischen Survey-Studie besteht in der praxisnahen Erforschung der realen Ablaufbedingungen bzw. der direkt im Prozess beobachtbaren Wirkungen von Psychotherapie bzw. Coaching (Fäh \& Fischer, 1998, S. 45), und je nach Bedarf können Zusammenhänge von Wirkungsweisen beispielsweise durch den Vergleich zwischen erfolgreich und weniger erfolgreich verlaufenden Prozessen überprüft werden.

Die Erhebung der Daten für die Kasseler Coachingstudie erfolgte im Rahmen einer fragebogenbasierten Prä-, Post- und Katamnese-Befragung von Coachs und Coachees, bei der unter anderem Skalen für die Maße Zieldefinition und Zielerreichung, Nutzen des Coachings, Selbstreflexion \& Selbsterkenntnis und Arbeitsbündnis zum Einsatz kamen. Ergänzt wurde der Fragebogen um offene Fragen zu Zielen, erreichten Ergebnissen und „kritischen“ Momenten (positiv wie negativ) im Prozess. Parallel, und für die Themenstellung und Herangehensweise dieser Arbeit von zentraler Bedeutung, wurde ein Teil der zu beforschenden Coachingprozesse vollständig audiographiert, so dass die Gesprächsverläufe detailliert ausgewertet werden können. 


\subsection{Aufbau dieser Arbeit}

Der Aufbau dieser Arbeit orientiert sich im Wesentlichen an dem von Mayring (2001) vorgestellten gemeinsamen Ablaufmodell für qualitative und quantitative Forschung. Es besteht aus insgesamt sechs Schritten:

1. Explikation und Spezifizierung der Fragestellung: Relevanz, Problembezug der Fragestellung, Hypothesen oder offene Fragestellung

2. Explikation des Theoriehintergrunds: Stand der Forschung, Theorieansatz, Vorverständnis

Diese ersten beiden Schritte können als eng zusammengehörend verstanden werden, so dass im folgenden Kapitel 2, nachdem die Fragestellung oben bereits skizziert wurde, zunächst auf den Erkenntnisstand im Blick auf Coaching und speziell im Blick auf Veränderungsprozesse im Coaching einzugehen sein wird. Dieser eher allgemeine Teil soll den Stand der Coachingforschung in aller Kürze darstellen und verzichtet bewusst auf Details, die für die Fragestellung dieser Arbeit von untergeordneter Bedeutung sind. Ausführlicher sollen dann in Kapitel 3 die theoretischen Grundlagen experienziellen Erlebens und Vorgehens betrachtet werden. Zum besseren Verständnis soll dabei auch die methodische Umsetzung des Focusing beschrieben werden.

Vor dem Hintergrund, dass Gendlin es weitgehend unterlässt, seinen Ansatz mit allgemeinpsychologischen, kognitiven oder Emotionstheorien in Beziehung zu setzen, soll in dieser Arbeit auch ein eigenständiges Kapitel (Kapitel 4) für einen Diskurs verwendet werden, um Bezüge beziehungsweise Abgrenzungen zwischen Gendlins Konzepten und aktuellen körperzentrierten Ansätzen, neurowissenschaftlichen Erkenntnissen und ausgewählten psychologischen Konzepten zur intrapersonalen Veränderung aufzuzeigen und damit auch einer kritischen Auseinandersetzung mit dem experienziellen Konzept Raum zu geben.

Die weiteren Schritte nach Mayring (ebd.) sind:

3. Empirische Basis: Beschreibung der Stichprobe, Beschreibung der Materialauswahl bzw. des Materials

4. Methodischer Ansatz: Erhebungs-, Aufbereitungs-, Auswertungsverfahren, Begründung der Verfahren

5. Ergebnisse: Darstellung, Zusammenfassung, Analyse, Rückbezug auf Hypothesen bzw. Fragestellung

In der Praxis der Forschung bestimmt der methodische Ansatz auch die Auswahl der Datenbasis, so dass Festlegungen zur Materialauswahl in einer gewissen Abhängigkeit zur Auswertungsmethode stehen. So sollen in Kapitel 5 zunächst die methodischen Grundlagen des Forschungsvorhabens beschrieben werden, und hier insbesondere auch die Methodik der zum Einsatz kommenden Task Analyse. Kapitel 6 widmet sich dann der empirischen Basis und direkt darauf aufbauend der konkreten Umsetzung der Task Analyse. Dazu gehört auch, ausgehend vom theoretischen Vorverständnis ein theoretisches Aufgabenmodell abzuleiten, es 
näher zu beschreiben und mit den entsprechenden empirischen Befunden abzugleichen. Dabei wird damit umzugehen sein, dass der Begriff „Felt Sense“ nicht klar definiert und damit nur schwer operationalisierbar bzw. einer empirischen Überprüfung nur schwer zugänglich ist (Sachse et al, 1992, S. 12).

Kapitel 6 endet mit einem theoretisch-empirischen Modell intrapersonaler Veränderung auf Basis der empirischen Daten und einem Rückbezug dieses Modells auf die theoretischen Grundlagen. Im 7. Kapitel schließen sich dann Implikationen für die Praxis an, sie enthalten theoretische und praktische Hinweise zum experiencingorientierten Coaching.

Als abschließende Elemente nennt Mayring (ebd.):

6. Schlussfolgerungen: Gütekriterien, Relevanz der Ergebnisse

Entsprechend wird Kapitel 8 im Rahmen der methodischen Reflexion auf die Gütekriterien und auch auf die Limitierungen dieser Arbeit eingehen, bevor dann in Kapitel 9 ein abschließendes Fazit gezogen wird.

\subsection{Sprachliche Regelungen}

Die in dieser Arbeit gemachten Ausführungen beziehen sich auf Personen unabhängig von ihrem jeweiligen Geschlecht. Geschlechtsspezifische Unterscheidungen sind für die Fragestellung von untergeordneter Bedeutung. Um die Lesbarkeit der Arbeit zu erleichtern, hat der Autor sich entschieden, in einzelnen Kapiteln die männliche Sprachregelung anzuwenden, also von Klienten, Beratern etc. zu sprechen, und in anderen Kapiteln die weibliche Sprachregelung anzuwenden, also von Klientinnen, Beraterinnen etc. zu sprechen. Gemeint sind in diesen Fällen auch Personen des jeweils nicht explizit erwähnten Geschlechts.

In Zitaten wird jeweils die im Original gewählte Sprachregelung wiedergegeben.

\subsection{Umgang mit Anlagen}

Die Durchführung der Studie, insbesondere die Vorbereitung der Task Analyse, erforderte die Erstellung zahlreicher Anlagen. Diese wurden der Arbeit in digitaler Form beigefügt. Eine Übersicht über die Anlagen wurde im Anhang beigefügt. Ebenfalls im gedruckten Anhang befindet sich eine Erklärung des Autors gemäß der Promotionsordnung der Universität Kassel. Aus Gründen des Datenschutzes sind die im Rahmen der Untersuchung erhobenen Original-Daten in Form von Audiographien, die Fragebogendaten und die Legenden zu den anonymisierten Transkripten nicht online verfügbar. 


\section{Coaching}

Ursprünglich im Sport und im Ausbildungssystem amerikanischer Universitäten angesiedelt, ist Coaching seit den 1990er Jahren ins allgemeine Bewusstsein gerückt, insbesondere im Kontext von personenorientierter Managementberatung im Sinn eines diskreten Reflexionsund Entwicklungsraums (Fietze, 2015, S. 3). Heute gilt das Format Coaching im arbeitsweltlichen Kontext als etabliert und ist entsprechend nachgefragt. Kauffeld \& Gessnitzer (2018, S. 15) sehen die Ursache für die zunehmende Verbreitung von Coaching in der sich dynamisch entwickelnden Arbeitswelt und den sich verändernden Arbeitsbedingungen, die steigende Anforderungen an das Individuum im organisationalen Kontext stellen und gleichzeitig eine gewisse Unsicherheit mit sich bringen.

Allerdings bleibt Coaching nach wie vor Gegenstand konzeptioneller Diskurse, die sich auch in unterschiedlichen Definitionen und Einordnungen in die Beratungslandschaft zeigen (Greif et al, 2018, S. 2). Vor diesem Hintergrund soll hier zunächst das dieser Arbeit zugrunde liegende Coachingverständnis aufgezeigt und der Forschungsstand, auf den sich die weiteren Ausführungen beziehen, dargestellt werden.

\subsection{Definition}

„Coaching ist eine intensive und systematische Förderung ergebnisorientierter Problem- und Selbstreflexionen sowie Beratung von Personen oder Gruppen zur Verbesserung der Erreichung selbstkongruenter Ziele oder zur bewussten Selbstveränderung und Selbstentwicklung.“" (Greif, 2008, S. 59)

Aufbauend auf Greifs Definition soll Coaching hier verstanden werden als eine dyadische, partnerschaftliche, arbeitsweltbezogene und prozessorientierte Beratungsdienstleistung, die Lern- und Veränderungsprozesse begleitet (Friesenhahn, 2017, S. 11) und darauf abzielt, die Klarheit, Handlungs- und Bewältigungskompetenz der Klientin zu fördern (Migge, 2007, S. 22; Maurer, 2013, S. 209, Ajdukovic, Cajvert, Judy, Knopf, Kuhn \& Madai, 2014, S. 54). Auch wenn Coaching Aspekte von Bildungs- und Lernarrangements beinhaltet (Lindart, 2016, S. 22), geht es in Abgrenzung zu Trainings- und Edukationsprogrammen im Coaching nicht primär um das Vermitteln von Wissen oder Fertigkeiten, sondern darum, dass die Coach für eine gewisse Zeit die Rolle einer begleitenden Expertin für selbstregulierte Problemlöseprozesse ihrer Klientin einnimmt (Ebner, 2016, S. 85).

Anlässe und Themen für Coaching sind typischerweise Probleme und Fragen der Kommunikation und zwischenmenschlichen Beziehungsgestaltung, hohe Arbeitsbelastung, berufliche oder organisationale Veränderung, strategische Positionierung und Entwicklung und Werte 
und Sinngebung (Loos, 2016, S. 45, Wilmes \& Loebbert, 2013, S. 17). Dabei verfolgen psychologische Coaching-Konzepte das Ziel, die Coachingklientin bei der Verbesserung ihrer Selbststeuerung und der Entwicklung von Handlungsalternativen zu begleiten und sie zu stärken, sich in ihrem Umfeld als souveräne Gestalterin zu bewegen. (Fischer-Epe, 2002, S. 22; Wegener, 2019, S. 15).

Im Coaching

„ereignet sich für den Klienten etwas für ihn Neues: Neue Handlungsmöglichkeiten und ungewohnte Sichtweisen werden eröffnet, die das Handeln des Klienten in seiner wirklichen Welt zum Erfolg führen.“(Wilmes \& Loebbert, 2013, S. 17)

Auch wenn im Coaching zu großen Teilen auf Konzepte zurückgegriffen wird, die ursprünglich im psychotherapeutischen Kontext entwickelt wurden, und wenn hier ebenso wie in der Psychotherapie die Persönlichkeit und die Arten des Fühlens, Denkens und Handelns der Beteiligten thematisiert werden (Roth, 2019, S. 298), richtet sich Coaching doch grundsätzlich an gesunde Personen, die während des gesamten Coachingprozesses eigenständig agieren und in ihrer Selbstverantwortung unterstützt werden. Insofern ist Coaching abzugrenzen von der Psychotherapie oder Beratung psychischer Störungen (Limmer \& Schütz, 2018, S. 486; Greif, 2008, S. 59).

An anderen Stellen ist die Abgrenzung des Formats weniger eindeutig: Fietze (2015, S. 12) weist auf die Abgrenzungsschwierigkeiten gegenüber Beratungsformen wie Supervision, Organisationsentwicklung, Mediation oder Mentoring hin, die sich ebenfalls als reflexionsfördernde und prozessorientierte Beratung definieren lassen. Diese Abgrenzungsfragen werden ausführlich im Zusammenhang der Professionalisierungsbestrebungen von Coaching diskutiert (vgl. Gessnitzer \& Kauffeld, 2018, S. 19; Greif et al, 2018, S. 2) und sollen, da sie für die Fragestellung dieser Arbeit eine untergeordnete Relevanz haben, hier nicht weiter vertieft werden.

Ergänzend zeigen Greif et al (2018, S. 5) den Einfluss unterschiedlicher Coachingkonzepte und unterschiedlicher psychologischer Schulen auf das jeweilige Coachingverständnis auf. Soweit diese Theoriesysteme und Konzepte eine Relevanz für die Themenstellung dieser Arbeit haben, werden sie später noch aufzugreifen sein.

\subsection{Coachingforschung}

Generell kann für die Coachingforschung gesagt werden, dass sie sich hinsichtlich der Verfahren zur Untersuchung von Coachingprozessen in weiten Teilen an den für die Psychotherapieforschung entwickelten Vorgehensweisen orientiert (Roth, 2019, S. 298). So sind für das Coaching Antworten auf die Fragen nach der Wirksamkeit und den Ergebnissen ebenso von Bedeutung wie Erkenntnisse über das Prozessgeschehen, das zu Veränderung oder Entwicklung führt. 
$\mathrm{Zu}$ Beginn der 2010er Jahre wurde die Coachingforschung noch in weiten Teilen als am Anfang stehend beschrieben (Möller \& Kotte, 2011, S. 446). In den letzten Jahren wurden die Forschungsvorhaben nun deutlich intensiviert, so dass heute bereits eine Fülle empirischer Untersuchungen zum Coaching zu verzeichnen ist, die insbesondere Aussagen zur Ergebnisqualität von Coaching ermöglichen (Kotte, Hinn, Oellerich \& Möller, 2018, S. 554). Die Herausforderung für die Coachingforschung besteht allerdings nach wie vor in der Vielfalt der Verwendung des Coachingbegriffs und der Anwendungsfelder von Coaching (vgl. Böning \& Kegel, 2015). De Haan \& Mannhardt (2014) weisen darauf hin, dass die Bewertung von Coaching auch vor dem Hintergrund einer hyperkomplexen Welt als sehr komplex zu betrachten ist und daher auch die Forderung nach qualitativer Coachingforschung Auftrieb erhält, um bisher unbekannte oder verborgene Phänomene sichtbar zu machen und coachingbezogene Theorien und Modelle weiterzuentwickeln.

\subsubsection{Wirksamkeit, Outcome}

"The research clearly shows that coaching has great potential to enhance the performance, productivity and well-being of individuals, organizations and the people that work for them." (Grant, 2014, S. 338)

In ihrer Zusammenfassung der zentralen Ergebnisse der Metaanalysen von De Meuse, Dai \& Lee (2009), Jones, Woods \& Guillaume (2015), Sonesh, Coultas, Lacerenza, Marlow, Benishek \& Salas (2015) und Theeboom, Beersma \& van Vianen (2014) kommen Kotte et al (2018, S. 555) zu dem Ergebnis, dass Coaching insgesamt wirkt, wobei eine starke Bandbreite an Effekten zu verzeichnen ist. Je nach Studie und Kategorie zeigen sich geringe, mittlere oder große Effekte zwischen Hedges' $\mathrm{g}=.10$ und $\mathrm{g}=.74$ (Kotte, Hinn, Oellerich \& Möller, 2016, S. 14). Die Effekte beziehen sich sowohl auf die subjektive Einschätzung der Coachee zum Erfolg (Reaktionsebene) als auch auf die Lernebene, die Verhaltensebene und Ergebnisebene (Kotte et al, 2018, S. 557).

Der allgemeine Nutzen und die positiven Effekte von Coaching lassen sich wie folgt zusammenfassen (Kotte, Oellerich, Schubert \& Möller, 2015, S. 36; Künzli, 2013, S. 370; Theeboom et al, 2014; Ebner, 2016, S. 86, Athanasopoulou \& Dopson, 2018, S. 75):

- Reduzierung negativer Belastungseffekte und Zunahme allgemeinen Wohlbefindens durch Coaching

- Zunahme von Arbeitszufriedenheit und Karrierezufriedenheit

- Effektivere Führung und Zielerreichung

- Veränderungen im Umgang mit Konflikten, Selbstbewusstsein und Selbstakzep$\tan z$

- Entwicklung von Bewältigungsstrategien und Kompetenzen

- Stärkung selbstregulativer Strategien und der Kompetenz- und Kontrollüberzeugung

- Positive Auswirkungen auf die Arbeitsleistung und Produktivität. 
Im Vergleich zum Training wird der Vorteil von Coaching darin gesehen, dass es auf individuelle Bedürfnisse eingeht, selbstgesteuertes Lernen und selbstgesteuerte Entwicklung fördert und unmittelbar im Alltag Umsetzung finden kann (Kotte et al., 2016, S. 14). Allerdings ist die Wirksamkeit von Coaching nicht generell gegeben, sondern ist abhängig von der jeweiligen Coaching-Intervention bzw. vom Vorhandensein der jeweils relevanten Wirkfaktoren (Kotte et al, 2018, S. 558).

\subsubsection{Wirkfaktoren, Prozess}

„Es sollte klar sein, wodurch Coaching wirkt, beziehungsweise welche Faktoren zur Wirksamkeit von Coaching beitragen, damit diese gezielt bedient werden können und eine Beratungsintervention den (...) Nutzen bestmöglich entfalten kann.“ (Lindart, 2016, S. 19)

Ein Pionierwerk der Auseinandersetzung mit Wirkfaktoren im Coaching ist das „Model of Coaching Effectiveness" von Kilburg (2001). Es basiert im Wesentlichen auf Erkenntnissen der Gesundheits- und Psychotherapieforschung, die auf das Coaching übertragen wurden. Kilburg beschreibt sieben Faktoren, die sich teilweise auf die Coach, teilweise auf die Coachee oder auf die Rahmenbedingungen des Coaching beziehen und effektives Coaching kennzeichnen (vgl. Lindart, 2016, S. 41):

1. Commitment von Coach und Klientin: Die Bereitschaft, Hingabe oder Verpflichtung dem Entwicklungs- bzw. Coachingprozess gegenüber

2. Charakteristika des Problems: Gemeint ist damit z.B. dessen Komplexität, Intensität, Dauer oder Emotionalität

3. Strukturelle Rahmenbedingungen wie die Klarheit und Verbindlichkeit der Absprachen zur Zusammenarbeit und den zu erreichenden Zielen

4. Arbeitsbeziehung: hierunter zählen auch die coachseitigen Eigenschaften bzw. Fähigkeiten zur Gestaltung des Arbeitsbündnisses wie Empathie, Authentizität, Toleranz, Wertschätzung

5. Qualität der Interventionen: Auswahl und Angemessenheit der Interventionen

6. Adhärenz: Einhaltung bzw. Umsetzung der im Coaching vereinbarten Maßnahmen

7. Organisationaler Kontext wie finanzielle Ressourcen oder die Unterstützung durch Vorgesetzte.

Greif (2008) entwickelte ein Strukturmodell der Wirkungen beim ergebnisorientierten Einzelcoaching, das teilweise auf Erkenntnissen der Psychotherapieforschung basiert, jedoch auch weitere, im Rahmen der Coachingforschung gewonnene Erkenntnisse beinhaltet. Er versteht sein Modell als „eine theorieorientierte Zusammenfassung zum Stand der Forschung und praktischer Beobachtungen“" (ebd., S. 275) und beschreibt folgende Wirkfaktoren:

- Wertschätzung und Unterstützung: verbale und nonverbale Verhaltensweisen, mit 
denen Empathie, Wertschätzung und emotionale Unterstützung ausgedrückt werden

- Affektreflexion und -kalibrierung: Reflexion und Regulierung von starken Gefühlsregungen

- Ergebnisorientierte Problemreflexion

- Ergebnisorientierte Selbstreflexion als Kernmerkmal von Coaching

- Zielklärung

- Ressourcenaktualisierung: interne Ressourcen umfassen hilfreiche Persönlichkeitsmerkmale der Klientin, externe Ressourcen liegen außerhalb der Klientin bzw. in ihrem Umfeld

- Umsetzungsunterstützung: Unterstützung beim Alltagstransfer der Klientin

- Evaluation im Verlauf durch Feedbackprozesse zwischen Coach und Klientin

- Individuelle Analyse und Anpassung entsprechend der jeweiligen Persönlichkeit der Klientin und ihres Kontexts.

Folgt man den Erkenntnissen der Psychotherapieforschung, muss davon ausgegangen werden, dass auch im Coaching die relevanten Wirkfaktoren eher methodenunspezifisch sind, und dass allgemeine Aspekte wie Beziehung, Motivation, Involvierung und Erwartung in ihrer Bedeutsamkeit und Wirksamkeit eher unterschätzt werden (Czogalik \& Enke, 1997, S. 511). Bezogen auf die Therapie stehen Interventionen nach Einschätzung von Czogalik \& Enke (ebd.) dann in einer konstruktiven Beziehung zum Prozess, wenn sie in der Lage sind, auf dem Fundament einer tragfähigen Therapeutin-Patientin-Beziehung eine integrierbare Neuerfahrung und Neubewertung bei der Patientin anzustoßen oder zu vertiefen. Bezogen auf Coaching bestätigen verschiedene Studien (vgl. de Haan \& Mannhardt, 2014, Mäthner, Jansen \& Bachmann, 2005; Kotte et al, 2016, Kotte et al, 2018) ebenso die hohe Bedeutung der Wirkfaktoren Beziehung und Arbeitsallianz für ein positives Ergebnis.

Graßmann, Schölmerich \& Schermuly (2019) unterstreichen mit ihrer Metaanalyse über 27 Stichproben und $\mathrm{N}=3563$ Coachingprozesse die hohe Bedeutung der Beziehungsqualität im Coaching: Die metaanalytischen Ergebnisse deuten auf einen moderaten und konsistenten Zusammenhang zwischen einer qualitativ hochwertigen Arbeitsbeziehung und CoachingWirkungen hin $(r=.41, p<.001)$. Die Beziehungsqualität ist positiv mit allen wünschenswerten Coaching-Wirkungen verbunden $(\mathrm{r}=.32$ bis .64), mit dem stärksten Zusammenhang zu affektiven und kognitiven Coaching-Wirkungen wie z.B. Zufriedenheit mit dem Coaching oder Wissenserwerb. Darüber hinaus ist die Beziehungsqualität negativ mit unbeabsichtigten negativen Auswirkungen des Coaching verbunden ( $\mathrm{r}=$-.29). Der Zusammenhang zwischen Beziehungsqualität und Coaching-Wirkungen wird nicht beeinflusst von der Anzahl der Coaching-Sitzungen oder der Perspektive derjenigen, die Beziehungsqualität und Wirkungen einschätzen, also ob die Beurteilung aus Sicht der Klientin oder der Coach erfolgt.

Als weiterer zentraler Aspekt der Wirksamkeit tritt die Zielklärung hervor: eine elaborierte Zielformulierung und Kontrolle der Zielannäherung wirkt sich erkennbar positiv auf das 
Coachingergebnis aus (vgl. Mäthner et al, 2005; Möller \& Kotte, 2011).

In ihrer Metaanalysen-Übersicht zum Coaching merken Kotte et al (2016) an, dass der Coachingerfolg bei der von vielen Studien präferierten Selbsteinschätzung signifikant höher liegt als bei einer Fremdeinschätzung, so dass hier noch Klärungsbedarf besteht. Auch Deplazes, Schwyter \& Möller (2016) weisen darauf hin, dass die Datenlage der Coachingforschung insgesamt noch eher dünn und unsystematisch ist, so dass detaillierte Aussagen zum Coachinggeschehen nur mit Einschränkungen getroffen werden können. Dies betrifft insbesondere das konkrete Handeln und Erleben im Coachingprozess, da die Forschung bisher auf die Frage fokussiert war, ob Coaching wirkt, und weniger auf die Frage, wie Coaching wirkt (ebd). Andere Autorinnen betonen ebenfalls die Notwendigkeit, Coachingprozesse auch inhaltlich nachzuvollziehen:

„Um den Einfluss und den Beitrag von Executive Coaching und anderer organisationeller Beratungsinterventionen zu verstehen, ist ein Wissen über ihre generelle Wirksamkeit und ihre Ergebnisse nicht ausreichend. Man muss auch die zugrundeliegenden Coaching-Prozesse selbst - sowohl aus Sicht der Klient/innen als auch der Coachs - erforschen und sie verstehen lernen.“ (Graf, 2015, zitiert und übersetzt nach De Haan et al, 2010)

In Analogie zur Psychotherapieforschung (Antal 2012, S. 27) ist hierfür eine differenzierte Einzelfallforschung notwendig, die die Interaktion zwischen Coach und Klientin, aber auch die jeweils individuellen Verhaltensweisen von Coach und Klientin und sogenannte Sub-Outcomes, also Wirkungen innerhalb einzelner Episoden oder Coaching-Sitzungen, untersucht (De Haan \& Nieß, 2012).

\subsection{Veränderung}

In Anlehnung an Kirkpatrick (1967) beschreiben Kotte et al (2018, S. 555) vier Ebenen der durch Coaching intendierten Veränderung:

„1. Reaktionsebene: subjektive Einschätzungen des Coachee zu Erfolg und Zufriedenheit,

2. Lernebene: Umfang, in dem angestrebtes kognitives Wissen und Strategien erworben sowie affektive Veränderungen (Einstellungen, Motivation) erzielt werden,

3. Verhaltensebene: Ausmaß, in dem das im Coaching Gelernte in den Alltag transferiert wird,

4. Ergebnisebene: Einfluss des Coachings auf organisationale Ziele, z.B. Arbeitsleitung oder Mitarbeiterbindung.“

Wenn sich im Coaching, wie in der Definition beschrieben, „Neues“ ereignen soll, das bisher nicht wahrgenommene Handlungsmöglichkeiten oder Sichtweisen eröffnet, lässt sich dieses Neue allen vier dieser Ebenen zuordnen, wobei ein Schwerpunkt der in einer jeweiligen Coachingsitzung oder Coachingsequenz stattfindenden Veränderung im Wesentlichen auf 
den Aspekten der intrapersonalen Veränderung liegt, also auf der Veränderung auf der Lernebene hinsichtlich der Einsichten und Einstellungen, des Wissens oder der Motivation. Diese Veränderung kann dann im Alltag auch zu einer Veränderung des Verhaltens und des organisationsbezogenen Ergebnisses führen.

Theorien zu intrapersonalen Veränderungsprozessen sind zunächst aus der klinischen Psychologie bzw. Psychotherapieforschung bekannt. Dort beziehen sie sich meist auf die Linderung oder Heilung psychischer Störungen, so dass Veränderungskonzepte und Störungskonzepte eng miteinander verwoben sind (Benecke, 2014, S. 32). Je nach Therapieschule unterscheiden sich die Veränderungskonzepte, indem sie beispielsweise abheben auf das „Ersetzen“ dysfunktionaler Kognitionen, die Entwicklung von Selbsttransparenz und Selbstkongruenz, die Affektkontrolle, die Entwicklung von Bindungsfähigkeit, die Entwicklung der Mentalisierungsfähigkeit oder Bewusstheit über innere Konflikte (ebd., S. 33). Als Bestandteile von Veränderungskonzepten benennt Benecke (ebd.):

- Annahmen über die Wirkprozesse, aufbauend auf den jeweiligen psychologischen Grundkonzepten

- Veränderungsstrategien: dazu gehören die inneren und äußeren Bedingungen der Veränderung, eine Theorie der therapeutischen Beziehung einschließlich der Bedeutung der Therapeutinnenpersönlichkeit, Phasen-Konzepte zur Spezifizierung des Veränderungsverlaufs und konkrete Strategien wie z.B. Durcharbeiten unbewusster Konflikte, kognitive Umstrukturierung oder Förderung einer regressiven Übertragungsbeziehung

- Methoden: sie konkretisieren die Strategien und werden durch konkrete Interventionen / Techniken umgesetzt

- Wirkungen im Sinne eines Entwicklungsprozesses in Richtung der Veränderungsziele.

Neben der klinischen Psychologie beschäftigt sich auch die Entwicklungspsychologie mit Veränderung. Dort wird der Veränderungsbegriff eher im Sinn von Entwicklung benutzt (vgl. Faltermaier, Mayring, Saup \& Strehmel, 2002, S. 24). Die Veränderungsprozesse können sich sowohl auf körperliche als auch auf kognitive, emotionale oder soziale Dimensionen beziehen und finden als interaktives Geschehen zwischen Person und Umwelt statt. (ebd., S. 27)

Kottler (2014, S. 13) fasst die verschiedenen Konzepte der Veränderung in einem Phasenmodell zusammen und beschreibt fünf Aspekte bzw. Sequenzen der Veränderung:

1. Es besteht ein ausgeprägter Wunsch nach Veränderung, der durch eine Krise, ein Trauma oder einen Entwicklungsübergang ausgelöst wird

2. Es wird ein Schmerzniveau und Unbehagen erreicht, das nicht mehr ignoriert oder geleugnet werden kann

3. Ein Bewusstsein oder eine Einsicht entsteht, dass eine Veränderung notwendig wird

4. Es gibt einen schrittweisen Prozess, in dem aus der Einsicht eine konstruktive 
Handlung erwächst

5. Unvermeidliche Rückschläge werden überwunden.

Nach Riva (2015, S. 22) lassen sich die Veränderungskonzepte innerhalb dieses allgemeinen Phasenmodells, abhängig von dem jeweils zugrundeliegenden Theoriemodell, in zwei Gruppen aufteilen: die eine Gruppe beschreibt er als „,bottom-up“-Modelle, die andere Gruppe als „top-down“-Modelle. Im top-down-Modell steht zunächst das bewusste und möglichst rationale Explorieren und Analysieren im Vordergrund, auf dessen Basis Erklärungen und Lösungen für emotionale und verhaltensorientierte Aspekte gefunden werden können. Das bottomup-Modell, dem auch das im nächsten Kapitel vorgestellte Experiencing-Konzept zuzuordnen ist, stellt im ersten Schritt die Auseinandersetzung mit dem emotionalen Erleben in den Vordergrund und führt dann schrittweise zu veränderten Konzepten oder zu verändertem Verhalten. Auch wenn beide Modelle zunächst als sich gegenseitig ausschließend erscheinen, plädiert Riva (ebd.) in Anlehnung an Stanovich \& West (2000) dafür, beide Modelle nicht konkurrierend, sondern sich als gegenseitig ergänzend zu sehen.

Als kritischen Meilenstein der Veränderung betrachtet Riva (2015, S. 21) den Übergang zwischen der zweiten und der dritten Sequenz des Phasenmodells, also die Phase, in der ein Bewusstsein für die anstehende Veränderung erwächst. Er empfiehlt, dieser Phase besondere Aufmerksamkeit zu widmen.

Zusammenfassend können als zentrale Merkmale der Veränderung gelten:

- Veränderung ist gekennzeichnet durch eine Reduzierung der Distanz zwischen Zielen und der Realität.

- Diese Verringerung wird erreicht durch:

- a) eine intensive Auseinandersetzung mit der spezifischen Erfahrung, die den Konflikt erzeugt;

- b) eine Reorganisation dieser Erfahrung.

- Die Auseinandersetzung und die Reorganisation der Erfahrung kann sowohl intuitiv als auch rational erfolgen.

- Diese Art von Distanz-Reduzierung geschieht in einem komplexen, in Phasen verlaufenden Prozess. (Riva, 2015, S. 23)

In der Coaching-Prozessforschung, auf die später noch einzugehen sein wird, wird intraindividuelle Veränderung auch in Verbindung gebracht mit sogenannten Critical Moments (De Haan \& Nieß, 2012, 2018), Bedeutsamen Momenten (Wegener, 2019) oder Aha-Erlebnissen (Greif \& Riemenschneider-Greif, 2018), wie sie auch aus der Psychotherapieforschung bzw. der Innovative-Moments-Forschung (Gonçalves, Matos \& Santos, 2009; Gonçalves, Mendes, Ribeiro, Angus \& Greenberg, 2010; Mendes, Ribeiro, Agnus, Greenberg, Sousa \& Gonçalves, 2011) bekannt sind. Analog zur der aus der psychotherapeutischen Prozessforschung als „Typ-B-Forschung“ bekannten Sichtweise (Bastine, Fiedler \& Kommer, 1989, S. 7; Rice \& Greenberg, 1984, S. 10) wird hier das Geschehen im Coaching als sequentielles Geschehen betrachtet und nicht als in sich einheitlicher oder linearer Prozess. Infolgedessen wird davon 
ausgegangen, dass sich im Prozess besonders signifikante Momente identifizieren lassen, die von Coachs und ihren Klienten als Veränderungsmomente wahrgenommen werden. De Haan \& Nieß (2012, S. 201) umschreiben diese Critical Incidents oder Critical Moments, als „an exciting, tense or significant moment experienced during coaching sessions." Konkret geht es dabei um:

- Momente des Lernens, bei denen eine neue Einsicht entsteht oder neue Verbindungen oder Perspektiven aufgegriffen werden

- Eine positive oder negative Veränderung in Beziehungen

- Eine Handlung seitens der Klientin oder der Coach, die in diesem Moment eine hohe Bedeutung hat

- Eine besonders positive oder besonders negative Emotion auf Seiten der Klientin oder auf Seiten der Coach

- Aufkommende Zweifel, die eine hohe Bedeutung haben. (ebd., S. 202)

In dem von Riva postulierten Phasenmodell können diese signifikanten Momente sowohl den kritischen Übergang von Phase zwei zu Phase drei markieren, also ein entstehendes Bewusstsein für die Notwendigkeit einer Veränderung, als auch den Übergang von Phase drei zu Phase vier, in dem bereits mögliche weitere Schritte erkennbar werden.

Wegener (2019) greift mit seinem Konzept der Bedeutsamen Momente zurück auf das Konzept der Critical Moments und definiert:

„«Bedeutsame Momente» im Coaching sind besonders produktive Episoden eines Coachings, in denen ein Coachee mit Hilfe eines Coachs erfolgreich jene Teilaufgaben bearbeitet, die in einem produktiven Verhältnis zum Coaching-Anliegen und zum damit verbundenen Coaching-Ziel stehen. Entsprechend müssen folgende drei Bedingungen erfüllt sein, um von einem «Bedeutsamen Moment» im Coaching sprechen zu können: Erstens müssen Coachees während dieser Episoden mentale Veränderungsprozesse vollziehen. Zweitens sind diese mentalen Veränderungsprozesse Ausdruck der erfolgreichen Bearbeitung von Teilaufgaben, die in einem produktiven Verhältnis zum Coaching-Anliegen und zum damit verbundenen Coaching-Ziel stehen. Drittens müssen sie als Ko-Konstruktion in der Interaktion von Coach und Coachee entstehen." (ebd., S. 102)

Veränderung im Coaching geschieht nach diesem Verständnis dann, wenn Coachees im Rahmen der Interaktion mit der Coach psychologische Wirkungen erleben, bei denen sich für sie etwas zum Positiven wendet und bei denen sie mentale Veränderungsprozesse im Sinn der Ausbildung funktionaler Deutungs- oder Handlungsmuster durchlaufen (ebd., S. 98). Relevant ist zudem, dass Veränderung sich nach diesem Verständnis ebenfalls als sequentielles Geschehen zeigt, bei dem die Veränderung insgesamt sich aus der erfolgreichen Bewältigung von Teilschritten zusammensetzt.

Greif \& Riemenschneider-Greif (2018, S. 113) messen dem Entstehen neuer Einsichten im 
Coaching eine große Bedeutung bei. Das Entstehen neuer Einsichten bezeichnen sie in Anlehnung an Beeman \& Kounios (2015) auch als Aha-Erlebnisse und meinen damit

,,innerpsychische Vorgänge, die sich in verbalen Äußerungen im Coaching-Prozess ausdrücken und in Handlungen der Klienten/Klientinnen im Coaching-Gespräch oder danach als Ergebnisse des Coachings niederschlagen können.“ (ebd., S. 114)

Damit also eine veränderte Handlung oder eine Verhaltensänderung entstehen kann, ist demnach zunächst eine veränderte Einsicht erforderlich, die die Betrachtungsweise oder Wahrnehmung einer Situation verändert. Die Autoren greifen in ihrem Konzept auf gestalttheoretische Überlegungen zurück, wonach es beim Aha-Erlebnis darum geht, eine bisherige sogenannte Fixierung zu lösen und von einer Betrachtungsweise oder Perspektive in eine andere $\mathrm{zu}$ wechseln und so neue Ressourcen oder bereits vorhandenes alternatives Wissen zu aktivieren. Auf diese Weise kann ein neues mentales Modell entstehen, das dann handlungswirksam werden kann, selbst wenn die Handlungspläne zu diesem Zeitpunkt noch nicht in jeder Einzelheit ausgearbeitet sind (ebd., S. 124).

Ryba (2019, S. 314) weist in diesem Zusammenhang darauf hin, dass aus neurowissenschaftlicher Sicht unbewusste und vorbewusst-intuitive Prozesse maßgeblich für das menschliche Erleben und Verhalten sind und dass hierbei somatisch-vegetative Prozesse eine wesentliche Rolle spielen. So fordert sie, dem körperlichen Erleben im Coaching mehr Aufmerksamkeit zu widmen:

„Für tiefgreifende Veränderungen bedarf es einer Berücksichtigung des Unbewussten. Dieses drückt sich weniger durch den Inhalt des Gesagten, sondern vielmehr durch die damit verbundenen vegetativ-somatischen Prozesse aus. Die Berücksichtigung des Körpers im Coaching ist aus diesem Grund ganz entscheidend.“ (ebd., S. 313) 


\section{Experiencing}

Auch wenn zum Focusing und seiner Umsetzung schon eine Reihe von Veröffentlichungen erschienen sind (Wiltschko, 2011, S. 95), scheinen die damit verbundenen Konzepte des Felt Sense bzw. des Experiencing noch wenig verbreitet. Zwar sind die Begriffe Focusing und Felt Sense mittlerweile auch im deutschen Sprachgebrauch als Fachbegriffe eingeführt (vgl. Sachse et al, 1992; Feuerstein \& Müller, 1998; Wiltschko, 2011), allerdings sind sie nach wie vor erklärungsbedürftig. Vor allem aber gilt diese Erklärungsbedürftigkeit für den Begriff des Experiencing. So spricht Hofmann (2016) von experienzieller Kommunikation, und Waldrich \& Schley (2019) sprechen von experienzieller Psychotherapie, während Feuerstein \& Müller (1998) Focusing zunächst als experienzielle Methode bezeichnen und später dann den Begriff erlebensbezogene Methode verwenden (Feuerstein \& Müller, 2000). Wiltschko (2011) spricht von der Theorie des Erlebens, und auch die deutsche Übersetzung von Gendlins (1996) Standardwerk „Focusing-orientierte Psychotherapie“ verwendet den Begriff der erlebensbezogenen Methode. Der Therapieansatz im engeren Sinn wird auch im deutschsprachigen Raum unter dem Terminus Focusing-Therapie weiterentwickelt (Wiltschko, 2011, S. 95). Die Uneinheitlichkeit der Begriffe mag bereits einen ersten Hinweis geben auf Schwierigkeiten, die mit der Darstellung des Konzepts einhergehen.

In dieser Arbeit werden die Begriffe erlebensbezogenes Konzept und experienzielles Konzept synonym verwendet, während der Begriff Focusing sich auf eine spezifische methodische Anwendung des experienziellen Konzepts bezieht. Zwar wird in der Literatur und auch von Gendlin als Begründer des Focusing diese Differenzierung zwischen dem theoretischen Konzept und der methodischen Anwendung nicht stringent vorgenommen, im Interesse der begrifflichen Klarheit scheint dem Autor eine solche Unterscheidung aber geboten.

Als Oberbegriff, der sowohl das Konzept als auch das durch Focusing intendierte intrapersonale Prozessgeschehen einschließt, benutzt diese Arbeit den Begriff Experiencing. Im englischen Original steht er sowohl für das Konzept an sich als auch für einen zentralen Aspekt des Konzepts, der den inneren Prozess beschreibt.

Ihre wesentliche Prägung erhielten Experiencing und Focusing durch Eugene Gendlin, den Schüler und späteren Nachfolger Carl Rogers an der Universität von Chicago, der im Rahmen seiner Psychotherapieforschungen vor allem der Frage nachging, wie Therapie zu langanhaltenden Veränderungen im Leben von Menschen beitragen kann. Seine zentrale Beobachtung war dabei die, dass für die Therapie relevante Veränderungsschritte hervorgerufen wurden durch etwas, was die Klienten innerlich, häufig intuitiv und unbewusst, taten. Dieses „Etwas“, das von außen zu beobachten war, bezog sich nicht nur auf das, was der Klient sagte, sondern auch darauf, wie er es sagte: 
„Die beste Prognose erhielt nicht der glatt und rasch sprechende Klient, sondern derjenige, der häufig schweigt, nach Worten sucht, um ein Gefühl zu beschreiben, der um Metaphern ringt, um sein Erleben zu schildern, dessen Körper ab und zu stärkere oder schwächere Veränderungen zeigt, wie z.B. auffallendes Ausatmen oder plötzliches Zusammensacken.“ (Wild-Missong, 1996, S. 7)

Gendlin machte sich im Folgenden zur Aufgabe, zu erforschen, was diese ,erfolgreichen Klienten“" anders machten als die „nicht erfolgreichen Klienten“. Sein Ziel war, diesen inneren Akt lehr- und lernbar zu machen. Hieraus entstand Focusing. Maßgeblichen Einfluss hatte dabei neben dem engen Bezug zu Carl Rogers und dessen Personzentrierter Theorie auch sein Studium der Philosophie und seine Beschäftigung mit der Strömung der Phänomenologie (Sachse et al, 1992, S. 6):

„Früh haben sich also in Gendlins Person und Leben philosophisches Denken und therapeutische Praxis gegenseitig gekreuzt und durchdrungen. Um diesen Prozess des Kreuzens von Denk- und Sprachformen mit der unmittelbaren Praxis geht es im Focusing (...) Welcher Art ist die Wechselwirkung zwischen dem körperlichen Erleben der immer beziehungshaften Situation und den Formen des Denkens, Sprechens und Handelns? Oder, populär ausgedrückt: Wie vollzieht sich die Verbindung zwischen „Kopf“ und „Bauch“ (oder „Herz“)? An dieser Schnittstelle, an der sich Denken und Fühlen kreuzen, ist Focusing zu Hause. (...) Gendlins Philosophie beansprucht nichts weniger, als den postmodernen Relativismus zu überwinden und die Phänomenologie neu zu begründen.“ (Wiltschko im Vorwort zu Gendlin \& Wiltschko, 1999, S. 7-8)

\subsection{Psychologische und philosophische Grundlagen}

Grundlage des Focusing ist die von Gendlin entwickelte Theorie des Experiencing, die aus psychologischer Sicht auf zentrale Grundannahmen des Personzentrierten Ansatzes zurückgreift, und aus philosophischer Sicht auf Annahmen der Existenzphilosophie und der Phänomenologie (Sachse et al., 1992, S. 7).

Die Humanistische Psychologie und der Personzentrierte Ansatz sind geprägt von einem tiefen Vertrauen in die Entwicklungsmöglichkeiten des Menschen und des Lebens und der Haltung. Bei Beratung und Therapie geht es nicht darum,

„ein einzelnes Problem zu lösen, sondern dem Individuum zu helfen so zu wachsen, dass es mit gegenwärtigen und zukünftigen Problemen in irgendeiner Weise besser umgehen kann.“ (Hinz \& Behr, 2002, S. 203)

Analog zum Menschenbild der Humanistischen Psychologie und aufbauend auf gestalttheoretische Überlegungen beschreibt Rogers (1972; 1991b; 2000; 2008) ein Persönlichkeitskonzept, dessen wesentliches Element die Aktualisierungstendenz ist: die von Natur aus dem Menschen innewohnende Neigung, sich konstruktiv in Richtung Selbstverwirklichung und 
Unabhängigkeit zu entwickeln (Weinberger, 1994, S. 97; Biermann-Ratjen \& Eckert, 2017, S. 36). Impulse zur Entwicklung müssen also nicht primär von außen gesetzt werden, sondern entwachsen der Aktualisierungstendenz des Menschen.

Rogers (2000, S. 42) unterscheidet zwischen dem Erleben - hierfür verwendet er den Terminus Experiencing -, welches durch ein biologisches, organismisches Wertsystem bestimmt wird und dem Konstrukt des Selbstkonzepts, das durch die Erfahrungen, die eine Person mit sich und den Prägungen der Umwelt macht, entwickelt wird. Solange das Selbstkonzept im Einklang mit dem organismischen Erleben steht, ist der Mensch ohne inneren Konflikt, er ist kongruent (Weinberger, 1994, S. 98) und kann sich weiter in Richtung Selbstverwirklichung und Unabhängigkeit entwickeln. Tritt jedoch eine Diskrepanz zwischen dem Erleben des Organismus und dem bewussten Selbstkonzept auf, kommt es zur Inkongruenz, zum zunächst oft nicht bewussten inneren Konflikt. Dieser Konflikt beeinflusst die Wahrnehmung, behindert die Entfaltung des Organismus und führt zu psychischen und physischen Spannungen.

Im beraterischen oder therapeutischen Prozess gilt für den Klienten deswegen, sich im Rahmen der Selbstexploration des organismischen Erlebens und dessen kognitiven Bedeutungsinhalts gewahr zu werden, um eine Verbindung mit dem Selbstkonzept herzustellen und so zu einem neuen Bewusstsein über die eigene Person und zu einem veränderten Selbstkonzept zu gelangen. Idealziel dieses Prozesses ist die „fully-functioning-person“, quasi eine „optimal entwickelte, hypothetische Persönlichkeit“ (Benecke, 2014, S. 188), die sich ihrer eigenen organismischen Erfahrungen gewahr ist und diese umfänglich zur eigenen Weiterentwicklung nutzen kann, indem sie ihr Selbstkonzept in Übereinstimmung mit diesen Erfahrungen bringt (Rogers, 1991b, S. 59).

Neben der Grundvoraussetzung eines psychologischen Kontakts zwischen Klient und Therapeut oder Berater, der beiden Seiten ermöglicht, sich gegenseitig wahrzunehmen und auch kognitiv und emotional zu interagieren, identifiziert Rogers drei Bedingungen als ausschlaggebend für den Erfolg einer Therapie oder Beratung (Rogers, 2000, S. 23ff):

1. Echtheit des Beraters (Kongruenz): Echtheit erfordert seitens des Beraters ein Offensein für das eigene Erleben im Kontakt mit dem Klienten und die Fähigkeit, dieses eigene Erleben auch in der Beratung zu nutzen.

2. Nicht an Bedingungen gebundene Akzeptanz (Wertschätzung): Die Zuwendung des Beraters soll gleichzeitig frei sein von Beurteilungen und Bewertungen der Gedanken, Gefühle und Verhaltensweisen des Klienten. Die Wertschätzung des Gegenübers ist nicht an Bedingungen geknüpft.

3. Präzise einfühlendes Verstehen (Empathie): Das innere Erleben des Klienten und die dabei latent oder deutlich vorhandenen Gefühle spielen in der Beratung eine wesentliche Rolle. Aufgabe des Beraters ist es, sich in den inneren Bezugsrahmen des anderen einzufühlen, quasi in der Innenwelt des Klienten zu Hause zu sein, ohne jedoch das Bewusstsein dafür zu verlieren, dass dies lediglich einer ,als ob“Position entspricht und nicht zum Zustand der Identifikation führen darf. (Rogers, 
1991b, S. 37)

Das Vorhandensein dieser drei Voraussetzungen schafft nach Rogers einen Beziehungsrahmen, der Selbstexploration ermöglicht und in dem die Aktualisierungstendenz zu Entwicklung, Wachstum und Reife führen kann. Personzentrierte Beratung versteht sich dem entsprechend als nicht pathologisierend, Probleme werden nicht als Defizite beschrieben, der Blick auf die Ressourcen und das Wachstum bleibt erhalten (Höher \& Steenbuck, 2006, S. 96).

Selbstexploration im personzentrierten Sinn meint die aktive und klärende Auseinandersetzung mit dem inneren Erleben und dessen Bedeutung, um so indirekte, bisher nicht wahrgenommene Lösungskompetenzen zu aktualisieren. (Sander \& Ziebertz, 2010, S. 86):

„Unter Selbstexploration soll verstanden werden, dass der Klient über sich selbst, über seine spezifischen persönlichen inneren Erlebnisse spricht, sich über sie klarer wird oder sich wenigstens deutlich um Klärung bemüht.“ (Tausch \& Tausch, 1973, S. 243)

Nach dem personzentrierten Konzept geht die Selbstexploration auch einher mit einem $\mathrm{Zu}-$ gewinn an emotionalen Selbstregulationsfähigkeiten und damit einer Zunahme an emotionaler Kompetenz (Maurer, 2006, S. 146).

Die in der Emotionspsychologie häufig anzutreffende Differenzierung zwischen Emotion als teilweise bewusste und teilweise unbewusste Reaktion des gesamten Organismus, die physiologische Erregung, Ausdrucksverhalten und Erfahrung in Form von Gedanken und Gefühlen beinhaltet (Ryba, 2018, S. 128), und Gefühl als bewusst erlebter Emotion (Benecke, 2014, S. 41) findet sich im personzentrierten Konzept insofern, als dass Fühlen verstanden wird als eine emotional gefärbte Erfahrung einschließlich ihres kognitiven Inhalts und ihrer persönlichen Bedeutung (Biermann-Ratjen \& Eckert, 2017, S. 27). Beide Begriffe sind dem Bereich des inneren bzw. organismischen Erlebens (Experiencing) zugeordnet, das es im Verlauf des Prozesses zu erkunden gilt (Rogers, 2000, S. 25). So werden Gefühle

,als sinnvolle und nützliche Information für den Organismus begriffen. Sie helfen uns dabei, herauszufinden, was für uns persönlich bedeutsam, gut oder schlecht ist. Sich ihrer bewusst zu sein, sie erleben und ausdrücken zu können, ist ein wichtiger Aspekt von Kongruenz und damit auch von psychischer Gesundheit.“ (Auckenthaler \& Helle, 2002, S. 150).

Sachse \& Langens (2014, S. 34) bringen den Begriff der Gefühle in Verbindung mit dem Begriff der Affekte, indem sie Gefühle als eine ,hochinferente, aber implizite (d.h. nicht vollständig explizierbare) Wissensform“ beschreiben, die komplexe Erlebens- und Wissensbestände verarbeitet. Das Ergebnis dieser Verarbeitung kann ein Affekt sein, also

„ein Zustand, in dem die Person eine (mehr oder weniger) diffuse körperliche Reaktion spürt, von der sie weiß, dass diese etwas (Wesentliches) bedeutet, diese Bedeutung jedoch nicht leicht entschlüsseln kann.“ (ebd.)

Die Verwendung des Emotionsbegriffs und des Affektbegriffs folgt in der Literatur teilweise uneinheitlichen Regeln (Benecke, 2014, S. 42), so dass hier zum besseren Verständnis noch 
eine begriffliche Einordnung vorgenommen werden soll: Nach Benecke (ebd.) sind Emotionen Prozesse, an denen verschiedene Subsysteme wie beispielsweise Bewertungsprozesse, motivationale Prozesse und Handlungsbereitschaften und auch neuronale und physiologische Prozesse beteiligt sind. Sie werden erlebt als mentale Zustände, die manchmal von körperlichen Veränderungen, von Ausdruckserscheinungen, oder auch von Handlungen oder Handlungsimpulsen begleitet werden.

Der Begriff Affekt wird in der Emotionspsychologie mit zwei unterschiedlichen Bedeutungen verwendet: zum einen wird er häufig als Synonym für Emotion gebraucht, vor allem dann, wenn es um die Betonung unbewusster Aspekte der Emotionen geht. Zum anderen wird er häufig ähnlich verwendet wie das Konzept „Stimmung“, also um einen Zustand von längerer Dauer, niedrigerer Intensität und geringerer Objektbezogenheit zu beschreiben (Sachse \& Langens, 2014, S. 16; Benecke, 2014, S. 41). Stimmungen werden meist als angenehm oder unangenehm (positiv oder negativ) erlebt, sind aber für die Person nicht mit starker physiologischer Aktivierung verbunden. Nach letzterem Konzept entstehen Affekte primär als Ergebnis präkognitiver Appraisalprozesse, während Emotionen eher als sekundäre Repräsentation der eigenen Innenwelt entwickelt werden und im Gegensatz zu den Affekten auch leichter benennbar, verstehbar und kontrollierbar sind (Sachse \& Langens, 2014, S. 30). Diesem Konzept folgend, ist auch eine Differenzierung zwischen affektiver und emotionaler Verarbeitung vorzunehmen. In beiden Verarbeitungsprozessen geht es zentral darum, die persönliche Relevanz eines Ereignisses oder eines Zustands einzuschätzen. Emotionale Verarbeitung zielt dabei auf die Feststellung von Qualitäten wie Bedrohung oder Verlust, damit möglichst schnell adäquat gehandelt werden kann. Die affektive Verarbeitung dagegen dient mehr der gründlichen, die indirekte Bedeutung und Komplexität berücksichtigenden Bewertung. Emotionale Verarbeitung ist nach diesem Konzept hoch explizit und kognitiv orientiert, während affektive Verarbeitung als eher implizit und perzeptuell-sensumotorisch orientiert betrachtet wird (ebd., S. 45).

Gendlin selbst verwendet mit dem Begriff des Felt Sense eine eigene Terminologie und grenzt diese gegenüber dem Emotions-Begriff ab (Gendlin, 1974, S. 228). Als Unterscheidungsmerkmal zwischen Emotion und Felt Sense benennt Gendlin (1996, S. 95), dass ein Felt Sense im Gegensatz zur Emotion weniger eindeutig erkennbar und schwieriger zu beschreiben ist, in der Regel aber mehr Bedeutungsinhalt hat als eine Emotion. Der Felt Sense

„,ist eine einzigartige Empfindung einer gesamten Subtilität und fällt somit nicht in eine uns vertraute Kategorie. Ein weiterer Unterschied zwischen einer Emotion und einem Felt Sense ist, dass eine Emotion weniger verlässlich ist als der Verstand, während ein Felt Sense verlässlicher ist als der Verstand. Wenn wir in Wut handeln, tut es uns später oft leid, weil wir nur auf einen Teil der Situation reagiert haben. Wenn wir ruhiger sind, erinnern wir uns der gesamten Situation. Das ist der Grund weshalb eine Emotion gewöhnlich weniger verlässlich ist als der Verstand. Im Ge- 
gensatz dazu ist ein Felt Sense verlässlicher als die Vernunft, weil darin mehr Faktoren gespürt werden können, als der Verstand verarbeiten kann. Das bedeutet nicht, dass wir im Hinblick auf den Felt Sense die Vernunft und eine verantwortungsvolle Auswahl aufgeben können. Im Gegenteil, wir brauchen immer sowohl den Felt Sense und die Rationalität.“ (ebd., S. 97)

Nach dieser Sicht bildet der Felt Sense quasi eine eigene Kategorie, die sowohl Emotionen beinhaltet als auch Affekte, aber auch kognitive Facetten wie Gedanken, Beobachtungen, Erinnerungen, Wünsche etc. Durch die Auseinandersetzung mit dem Felt Sense, durch das Fühlen, Symbolisieren und Explizieren, sollen dann die unterschiedlichen Aspekte, die der jeweilige Felt Sense beinhaltet, differenziert und erkennbar werden. So ist im Experiencing-Konzept der Begriff des Fühlens eng verbunden mit dem Begriff des Felt Sense und bezieht sich primär auf die Symbolisierung erlebter Bedeutung am Rand des Gewahrwerdens. Der Felt Sense ist damit auch weiter gefasst zu verstehen als der von Rogers geprägte Begriff des organismischen Erlebens, der in Gesprächspsychotherapie eine zentrale Rolle spielt.

\subsection{Gendlins Theorie des Experiencing}

Gendlin greift bei der Erarbeitung des Experiencing-Konzepts nicht primär auf psychologische Konstrukte zurück, sondern verknüpft seine Beobachtungen mit philosophischen Ansätzen zur Symbolisierung von Erleben und zum Zusammenhang von Erleben und Sprache. Wesentlicher Bezugspunkt sind hier die existenzphilosophischen Überlegungen von Heidegger, Jaspers, Sartre und Merleau-Ponty (Sachse et al, 1992, S. 7). Ein Grundgedanke dieser Überlegungen ist, dass die menschliche Existenz neben den individuellen Gedanken, Gefühlen und Repräsentationen der Umwelt noch weitere Aspekte beinhaltet, die als eine, ,inhaltlich nicht mehr fassbare Eigentlichkeit“ (ebd.) beschrieben werden. In Gendlins philosophischem Konzept sind diese Facetten der Existenz gleichbedeutend mit dem Selbsterleben des Organismus in seiner Interaktion mit der Umwelt. Sie enthalten eine Fülle präkonzeptueller, impliziter Aspekte, die im Focusing-Prozess durch den Felt Sense und die Symbolisierung erlebbar gemacht werden. Die Symbolisierung bzw. ihre gefühlte Bedeutung und die Suche nach Worten hierfür wird damit zu einem wesentlichen Element im vorwärtstragenden Prozess persönlicher Veränderung und Entwicklung (Feuerstein \& Müller, 1998, S. 42).

Nach Gendlins Konzept findet im Individuum ein kontinuierlich ablaufender Prozess der körperlich spürbaren Bezugnahme auf äußere Ereignisse statt. Dieser Prozess, den er als Experiencing bezeichnet, ist für ihn grundlegend für alle psychischen Ereignisse der Persönlichkeit (Sachse et al. 1992, S. 9). Dem entsprechend definieren Dahlhoff \& Bommert (1978, S. 65) Experiencing als

„das konkrete, im Augenblick vor sich gehende Erleben eines Individuums (...), bei dem die Aufmerksamkeit auf einen zugrunde liegenden gefühlten Erlebnisgegen- 
stand gerichtet ist. Dieser Gegenstand muss nicht unbedingt vollständig verbal fassbar sein, es ist für das Individuum mehr eine körperlich fühlbare Beziehung: eine gefühlte, unmittelbar gegenwärtige, persönliche Bedeutung von Dingen und Erlebnissen."

Focusing als Methode soll das Individuum dabei unterstützen, dieses gefühlsmäßige, oft als vorbegrifflich bezeichnete Erleben (ebd.) ins Zentrum der Aufmerksamkeit rücken zu lassen, so dass ein bewusster Prozess der Bezugnahme auf das innere Erleben stattfindet. Während des Fokussierens auf das innere Erleben im Sinn eines direkten Bezugsobjekts, entsteht demnach eine Art innerlich gefühlter Bedeutung, die Gendlin zunächst als Felt Meaning und später als Felt Sense bezeichnete (Sachse et al, 1992, S. 9; Dahlhoff \& Bommert, 1978, S. 67). Der Felt Sense wird damit als Bedeutungsträger konzeptualisiert, dessen implizite Bedeutung im Rahmen eines Focusing-Prozesses erfahrbar wird. In dieser Konzeption beinhaltet der Felt Sense zwar kognitive und emotionale Anteile, ist aber weder der Kategorie Denken noch der Kategorie Fühlen zuzuordnen, sondern wird als eigenständige, in gewisser Weise übergeordnete Kategorie, als „Erlebniskategorie, aus der sich Denken und Fühlen erst abspalten“, betrachtet (Sachse et al, 1992, S. 9, zitiert nach Wiltschko, 1979).

Gendlin (1978, S. 13) geht davon aus, dass der größte Teil des menschlichen Lebens innerhalb impliziter Bedeutungen verläuft, beispielsweise wenn gegenwärtige Reaktionen und Verhaltensweisen von zurückliegenden Erlebnissen geprägt sind, diese aber in unserer gegenwärtigen Reaktion nicht explizit präsent sind. Im Focusingprozess findet eine Interaktion mit dem Impliziten statt, bei der das innere Bezugsobjekt zum Bedeutungsträger wird. Wenn es dann gelingt, die gefühlte Bedeutung mit Wortsymbolen zu versehen, wird sie explizit. Ohne Symbolisierung bleibt die gefühlte Bedeutung unvollständig.

Im Konzept des Experiencing wird der Felt Sense nicht als statisch verstanden, sondern als Teil eines Prozesses, bei dem sich durch die bisher impliziten, nun aber explizierten Bedeutungsinhalte auch das Bezugsobjekt selbst verändern kann, so dass wiederum neue implizite Aspekte auftauchen. Veränderung findet demnach in einem permanenten und freien Interaktionsprozess zwischen implizit gefühlten Bedeutungen und äußeren Symbolen statt. Aus philosophischer Sicht formuliert Gendlin dazu:

„Jede gegenwärtige Erfahrung beinhaltet in der Tat vergangene, doch ist die gegenwärtige nicht einfach eine Neuanordnung vergangener Erfahrungen. Die Gegenwart ist ein neues Ganzes, ein neues Ereignis. Sie gibt der Vergangenheit eine neue Funktion, eine neue Rolle zu spielen. (...) Die Vergangenheit ändert sich in der neuen Gegenwart.“(Gendlin, 1996, S. 31)

Als zentrale Rahmenbedingung für einen solchen Prozess beschreibt Gendlin die soziale Gemeinschaft: Er geht davon aus, dass der Mensch nur in einer sozialen Interaktion kurzzeitig seine Existenz erleben kann (ebd., S. 8). Damit begründet er aus philosophischer Sicht die personzentrierten Parameter der Therapeut-Klient-Beziehung als Voraussetzung für gelin- 
gende Experiencing-Prozesse. Hier kann Implizites expliziert werden, hier findet tieferes Verstehen statt, das einen vorwärts tragenden Prozess ermöglicht.

„Wenn du verstanden hast, was ich sage, dann wirst du mehr verstanden haben als ich selbst. Denn jedes wirkliche Verstehen ist keine Kopie, sondern ein Weiterleben; es geht durch dich durch, durch deinen Körper, und wird von all dem, wer, was und wie du bist, fortgesetzt.“ (Wiltschko, 2017, S. 138, zitiert nach Gendlin, 1975)

Nach Gendlins Prozessverständnis hat eine solche Veränderung eine Richtung, die durch die Aktualisierungstendenz, also die dem Organismus innewohnende Tendenz zur Entwicklung seiner Möglichkeiten, vorgegeben wird. Hier ist zu unterscheiden zwischen der Richtung des Prozesses und einem definierten Ziel des Prozesses. Letzteres kann nicht vorab festgelegt, sondern nur Schritt für Schritt angepasst werden (Wiltschko, 2017, S. 141). Persönliche Entwicklung und Veränderung wird damit verstanden als eine ständige Abfolge von Interaktionsschritten zwischen geformten, bewussten Konzepten und dem unmittelbaren am Felt Sense orientierten Erleben. Dabei kommt dem Körper eine besondere Bedeutung zu, die sich nicht mit den gängigen Vorstellungen des Körpers als „physiologische Maschine“ (Gendlin, 1996, S. 12) beschreiben lässt, sondern bei der es darum geht, körperliches Erleben „,von innen“ (ebd.) wahrzunehmen und als implizite Quelle für einen Klärungsprozess zu nutzen.

\subsection{Die Focusing-Methode}

„Focusing ist eine Form nach innen gerichteter körperlicher Aufmerksamkeit (...) Es unterscheidet sich von der Aufmerksamkeit, die wir unseren Gefühlen normalerweise schenken, weil es - beginnend mit körperlichen Erfahrungen - in der Zone zwischen dem Bewussten und dem Unbewussten geschieht.“ (Gendlin, 1996, S. 11) In der beraterischen oder therapeutischen Praxis wird Focusing dann als sinnvolles Angebot erachtet, wenn bei der Bearbeitung eines Themas der Eindruck entsteht, dass da noch mehr sei, als im Augenblick erfasst werden kann, oder dass es um Aspekte geht, die momentan sprachlich nicht gefasst werden können (Sachse et al, 1992, S. 4). Focusing wird dabei nicht als eine eigenständige Therapierichtung oder Beratungstechnik verstanden, sondern als eine Methode, die beispielsweise innerhalb eines gesprächstherapeutischen Rahmens (Sachse et al, 1992, S. 5), eines verhaltenstherapeutischen Rahmens (Waldrich \& Schley, 2019) oder im Rahmen der kognitiven Therapie (Gendlin, 1996, S. 373) zum Einsatz kommen kann.

Gendlin (1996, S. 26) geht davon aus, dass Veränderungsschritte in einer Interaktion zwischen äußerem und innerem Erlebensgebiet bzw. in einer Grenzzone zwischen dem Bewussten und dem Unbewussten entstehen und mit Gedanken, Wahrnehmungen und einem direkt gespürten Körpererleben einhergehen. Wiltschko \& Renn (im Vorwort zu Gendlin, 1996, S. 7) bezeichnen diese Grenzzone als „Rand des Erlebens“ und als „Ort, an dem Neues auftaucht, Veränderung geschieht.“ Zentral ist dabei, die körperliche Wirkung des Themas zu erkennen und zunächst bei ihr zu verweilen. Gendlin (1996., S. 36) nennt es das „direkte Spüren in der 
Grenzzone“, eine physische, somatische Empfindung, die sich auf die Schicht des Unbewussten bezieht, die wahrscheinlich als nächstes an die Oberfläche kommt. Dabei entsteht ein körperlicher Bezugspunkt, den Gendlin „direct referent“ nennt bzw. „Felt Sense“ (ebd., S. 37) und der gespürt, aber in diesem Moment noch nicht näher spezifiziert oder interpretiert werden kann. Focusing besteht demnach zunächst darin,

„Zeit mit etwas körperlich Empfundenem, aber Unklarem zu verbringen (bis es ,in den Fokus“" kommt).“ (Gendlin, 1996, S. 59)

Im Rahmen dieses Verweilens bei dem körperlichen Empfinden sucht der Klient nach einem Symbol, das die gefühlte Bedeutung des körperlich Empfundenen möglichst adäquat abbildet. Wenn die aus dem Felt Sense heraus entstehende Symbolisierung gelingt, werden dem Klienten bisher unklare Aspekte eines Themas oder eines Problems verfügbar, die er in sein bewusstes Erleben integrieren kann (Sachse et al, 1992, S. 2). In der Regel empfindet der Klient dann eine unmittelbare Erleichterung, die Gendlin als Felt Shift bezeichnet. Ziel des Focusing ist damit die „Symbolisierung der gefühlten Bedeutung“( (ebd.), denn die gefühlte Bedeutung enthält nach diesem Verständnis auch Hinweise auf den Kern oder die Besonderheit des Problems bzw. auf ein Bedürfnis, das mit diesem Problem verbunden und noch nicht erfüllt ist, so dass sich aus dem Felt Sense sowohl die subjektive Bedeutung einer Problematik als auch deren Lösungsrichtung ableiten lassen (Feuerstein \& Müller, 1998, S. 42).

Um zum Felt Sense und Felt Shift zu gelangen, hat Gendlin eine konkrete Prozessanleitung ausgearbeitet, die sowohl das methodische Vorgehen des Focusing als auch den in der betreffenden Person vorgehenden Prozess beschreibt. Auch wenn Gendlin (1996, S. 271) betont, dass Experiencing bzw. der experienzielle Gebrauch von Methoden sich nicht auf Focusing begrenzt, ist doch das Verständnis des Focusing-Prozesses und der Focusing-Methode zunächst grundlegend. Das Vorgehen lässt sich anhand von sechs Schritten beschreiben, die in der Praxis fließend ineinandergreifen können (Gendlin, 1996, S. 114; Sachse et al, 1992, S. 3; Feuerstein \& Müller, 2000, S. 98):

1. Raum schaffen:

Ein Focusingprozess beginnt in der Regel mit einer Entspannungsübung, die dazu verhilft, innezuhalten und die Aufmerksamkeit auf den Körper und insbesondere auf die Körpermitte, den Brust- und Bauchraum zu richten und damit innerlich einen Freiraum zu schaffen. Dabei geht es um eine freundliche Zuwendung zu allen Empfindungen - sowohl den angenehmen als auch den unangenehmen. Gleichzeitig dient dieser Schritt dazu, die aktuellen Probleme zu sortieren, einen guten Abstand herzustellen zu bedrängenden Themen, Gedanken und Gefühlen und anschließend ein Thema, eine Fragestellung oder ein Problem herauszugreifen, das weiter bearbeitet werden soll.

2. Einen Felt Sense entstehen lassen:

Wenn die Aufmerksamkeit auf das ausgewählte Thema gerichtet ist, geht es darum, den „ungelösten Rand“ des Themas, also die impliziten Aspekte am Rand 
der Gewahrwerdung, zu adressieren. Ziel ist, das Wesentliche des Themas oder das Besondere am Problem innerlich zu symbolisieren. Dazu wandert die Aufmerksamkeit auf den Körper bzw. in den Körper, verbunden mit der Fragestellung, wo im Brust- und Bauchraum etwas zu diesem Thema reagiert. Dann gilt es, bei dieser reagierenden Körperstelle zu verweilen, quasi der vagen körperlichen Empfindung Gesellschaft zu leisten: der Felt Sense entwickelt sich, zunächst schlicht als körperliches Erleben von etwas, was noch nicht in Worte zu fassen ist.

3. Einen Griff finden:

Gemeint ist damit die Symbolisierung des Felt Sense, also ein Wort, eine Redewendung oder ein Bild, das die Qualität des Felt Sense möglichst genau wiedergibt. Durch diesen „Griff“ kann dem, was bisher noch nicht in Worte gefasst werden konnte, Ausdruck verliehen werden.

4. Die Stimmigkeit zwischen dem Griff und dem Felt Sense überprüfen:

Wenn die Symbolisierung den Felt Sense stimmig wiedergibt, verändert sich in der Regel auch das Körperempfinden, indem eine gewisse Erleichterung zu spüren ist. Möglicherweise bedarf es einer Nachjustierung des Griffs, also eines weiteren Abgleichs zwischen Symbolisierung und Felt Sense, um ein noch besser passendes Wort oder Bild zu finden, um den Felt Sense auch wirklich treffend in Sprache zu fassen. Der Symbolisierungsprozess dauert so lange, bis ein Felt Shift, eine körperlich fühlbare Empfindungsänderung, eintritt.

5. Fragen stellen:

Um den Felt Sense bzw. die Symbolisierung auf das Thema anzuwenden, werden nun Fragen gestellt, zunächst direkt an den Felt Sense, wie z.B.: Was bräuchte diese Stelle, um sich wohl(er) fühlen zu können? Welche Qualität fehlt dieser Stelle? Was ist das Schlimmste / Beste an dieser ganzen Sache? Dann können weitere Fragen folgen: z.B.: Macht es einen Sinn, das Wort oder das Symbol mit dem Thema in Verbindung zu bringen? Wird etwas klarer, wenn ich das Thema so betrachte? Zeigt sich eine Wirkung im Felt Sense, wenn er so befragt wird? Dabei ist auf ein enges Verweilen bei der Empfindung zu achten, theoretische und intellektualisierende Erklärungen sollen vermieden werden.

6. Empfangen und schützen:

Abschließend geht es darum, die Schritte des Focusing-Prozesses zu würdigen und das, was in der Beschäftigung mit dem Felt Sense entstanden ist, zu schützen, auch wenn das Ergebnis möglicherweise klein erscheint. Eigene innere Entwertungen des Prozesses sollen erkannt und Innere-Kritiker-Stimmen sollen gestoppt werden. Eine häufige Reaktion im Anschluss an einen Focusing-Prozess ist die, dass Klienten ihr eigenes inneres Erleben im Nachhinein kritisch reflektieren und als unangemessen, irrational, „kindisch“ einschätzen. Aufgabe des Therapeuten oder Beraters ist deswegen, nicht bei dieser „sekundären Reaktion“" zu verweilen, sondern dem Klienten zu helfen, auf die primäre Reaktion zu fokussieren, also das 
innere Erleben, das bis dahin nicht zugänglich für eine wertschätzend-freundliche Exploration war. Vor diesem Hintergrund ist dieser abschließende Schritt des Schützens wichtig, um das Ergebnis auch für weitere Schritte nutzen zu können (Rice \& Saperia, 1984, S. 40).

Focusing kann zwar auch von einem Individuum alleine durchgeführt werden, Gendlin (ebd., S. 136) empfiehlt aber, Focusing entweder dyadisch oder unter Anleitung durchzuführen, da so der Prozess besser unterstützt werden kann. Als Grundsätze für die therapeutische oder beraterische Unterstützung gelten (Gendlin, 1998, S. 140):

- Aktives Zuhören beim Focusing: gemeint ist damit das intensive und konzentrierte Zuhören, das Paraphrasieren und Verbalisieren der Klientenaussagen, ohne eine eigene Meinung einzubringen.

- Den Klienten einladen, zur Ruhe zu kommen, still zu werden, der eigenen Aussage im Körper nachzuspüren. Dazu können auch Fragen helfen, die einen Bezug zum körperlichen Erleben herstellen.

- Eigene Gefühle und Reaktionen, die im Blick auf den Klienten entstehen, diesem wertschätzend und akzeptierend zur Verfügung stellen.

\subsection{Experiencing-Forschung}

In der psychotherapeutischen Forschung werden Focusing und Experiencing den prozess- und erfahrungsorientierten Ansätzen (PE, ,process-experiential“) zugeordnet, unter deren Dach die humanistisch ausgerichteten Therapien der personzentrierten, gestaltorientierten oder existenziellen Psychotherapieschulen ebenso subsummiert werden wie weitere körperorientierte oder emotionsfokussierte Ansätze (Elliot, Greenberg \& Lietaer, 2013; S. 791). Gemeinsame theoretische Grundannahme dieser Ansätze ist das Verständnis des Menschen als freies Individuum, das sich nach Wachstum und Potenzialentwicklung ausrichtet. In der therapeutischen Praxis dieser Ansätze spielt das aktuelle Erleben, je nach Konzept mit einem eher emotionsorientierten oder phänomenologisch-symbolisierenden Fokus, eine wichtige Rolle, um einen Zugang zu mehr oder weniger bewussten organismischen Funktionen zu erhalten.

Einen metaanalytischen Überblick über 112 verschiedene Studien zu erfahrungsorientierten Therapien, supportiven Therapien, prozess-erfahrungsorientierten Vorgehensweisen, Gestalttherapie und anderen erfahrungsorientierten Therapieformen wie Focusing geben Elliot et al (ebd., S. 794). Im Prä-Post-Design über alle Studien hinweg berechnen sie eine Effektstärke (ES) von.99. Werden nur die Studien mit Kontrollgruppendesign $(\mathrm{N}=42)$ berücksichtigt, ergibt sich ein Prä-Post-Effekt von .89, so dass die Autoren zu dem Schluss kommen, dass die Wirksamkeit erfahrungsorientierter Therapien empirisch belegt ist (ebd., S. 849). Sie bestätigen damit ein Review von Cooper, Watson \& Hölldampf (2010) zur personzentrierten und experienziellen Therapie, das zu vergleichbaren Ergebnissen kam. Die Ergebnisse ihres Reviews über sechs Metaanalysen aus den Jahren 1980 bis 2008 fassen Elliot \& Freire (2010, 
S. 12) wie folgt zusammen:

„The overall findings, which have become increasingly clear with each successively larger data set, are uniformly good news for person-centred / experiential practitioners: Clients use our therapies to make larger changes in themselves; these changes are maintained over time and are much larger than our clients would have experienced without therapy. Furthermore, our clients show as much change as clients seen in other therapies."

Allerdings muss darauf hingewiesen werden, dass ein Teil der in den Analysen untersuchten Studien sich auf eine primär personzentrierte oder gestalttherapeutische Vorgehensweise bezog und die Autoren darüber hinaus betonen, dass noch ein großer Bedarf an Forschung besteht, um die Zusammenhänge von Denkprozessen, Verhalten und Erleben besser zu verstehen (Elliot, Greenberg \& Lietaer, 2019). Vor allem sollte die weitere Forschung spezifischen Klienten- oder Diagnosegruppen gelten, Standards für die jeweilige personzentrierte Intervention definieren und auch nach dem Standard randomisierter kontrollierter Studien (RCT) durchgeführt werden (Cooper et al, 2010, S. 243).

Einschränkend ist zu sagen, dass keine der Analysen eine Aussage zu Coachingprozessen oder arbeitsweltbezogener Beratung enthält, und dass Focusing in den Metaanalysen nur eine Facette unter vielen ist. Im Blick auf Experiencing und Coaching steht die Forschung weiter in den Anfängen, und letztlich kann derzeit lediglich auf Forschungserkenntnisse, die nicht explizit im Kontext von Coaching entstanden sind, zurückgegriffen werden.

Eine Übereinstimmung mit der allgemeinen Coachingforschung (sh. Kapitel 2.2.2) und der therapeutischen Forschung (Benecke, 2014, S. 634) ergibt sich hinsichtlich der Beziehungsqualität zwischen Therapeut bzw. Coach und Klient. Hier bestätigt sich die sowohl von Rogers als auch von Gendlin betonte Notwendigkeit einer positiven Beziehungsgestaltung und einer kongruenten, wertschätzenden und empathischen Haltung seitens des Therapeuten / Coachs als zentrale Rahmenbedingung für gelingende Therapie- oder Coachingprozesse. Eine Studie von Iberg (1996) weist ergänzend darauf hin, dass nicht nur eine personzentrierte Haltung, sondern auch eine experiencingorientierte Gesprächsführung wie z.B. das Einbringen von focusing-typischen Fragen einen positiven Beitrag zum Therapieerfolg leistet.

Als weiterer Aspekt kann zudem festgehalten werden, dass mehrere Studien darauf hindeuten, dass die spezifische Fokussierung auf Emotionen der Klienten mit positiven Behandlungsergebnissen korreliert (Benecke, 2014, S. 640). So kommt eine schulenunabhängig durchgeführte Psychotherapie-Metaanalyse von Diener, Hilsenroth \& Weinberger (2007) zu folgendem Ergebnis:

„Therapist facilitation of patient affective experience / expression was positively associated with treatment improvements. And this relationship most likely exists independently of the influence of other factors. The results suggest $30 \%$ difference in success rate between patients who receive affective therapeutic focus and those who 
do not (...) Results (...) indicate that therapist facilitation of patient affective experience / expression increased patient success from 35\% to 65\%." (Benecke, 2014, S. 641, zitiert nach Diener et al, 2007, S. 938)

Im Detail zeigt sich, dass insbesondere Interventionen, denen eine affektive Reaktion folgt, mit einem guten Therapieergebnis und einer dauerhaften Veränderung im emotionalen Bereich assoziiert sind. Der Veränderungsprozess sollte dabei allerdings nicht als linear verlaufend erwartet werden, sondern spielt sich vielmehr auf der Mikroebene in einer Art „2-stepsforward, 1-step-back fashion“ ab (Benecke, 2014, S. 643). Der Veränderungsprozess kann dabei sowohl im Rahmen der Aktivierung spezifischer relevanter Emotionen in den Sitzungen einschließlich der moderaten Aktivierung negativer, schmerzlicher Emotionen im (mittleren) Behandlungsprozess stattfinden als auch durch die kognitive Erkundung und Bearbeitung der Bedeutung dieser Emotionen.

„Günstig für den späteren Behandlungserfolg scheint insgesamt ein „moderate amount of expressed emotional arousal“ zu sein, während „too much or too little emotion was found not to be helpful.“ (...) Hohe Levels des Emotional Experiencing (...) zeigen einen robusten Zusammenhang mit dem Therapieerfolg (...) Im Verlauf von erfolgreichen Behandlungen steigt das Level des Emotional Experiencing bzw. des Emotional Processing deutlich an.“ (ebd.)

Um seine theoretischen Annahmen auch empirisch zu belegen, entwickelte Gendlin eine spezielle siebenstufige Experiencing-Skala (Klein, Mathieu, Gendlin \& Kiesler, 1969), die die Nähe des Klienten zu seinem inneren Erleben bzw. die Art, wie er sich diesem Erleben zuwendet, erfassen soll. In Stufe 1 der Skala schildert der Klient Ereignisse in allgemeiner, unbeteiligter Weise, in Stufe 3 bezieht er sich nur knapp und wie von einem äußeren Bezugspunkt betrachtet auf sein Fühlen und Erleben, in Stufe 4 bezieht er sich auf seinen inneren Bezugsrahmen, und in Stufe 7 kann der Klient klar und offen über sein unmittelbares inneres Erleben sprechen und die Bedeutung des Erlebens verstehen. Die Skala gilt als konsistent und reliabel (Dahlhoff \& Bommert, 1978, S. 79) und wird später noch im Detail vorzustellen sein (sh. Kapitel 4.6). Untersuchungen mit der amerikanischen Experiencing-Skala zeigen, dass ein hohes Experiencing-Niveau regelmäßig einhergeht mit einem signifikant positiven Therapie-Outcome, während Klienten in weniger erfolgreichen Therapieverläufen lediglich über ein niedriges Experiencing-Niveau verfügen (ebd.; Watson, Greenberg \& Lietaer, 2010, S. 139). Watson et al (ebd.) weisen darauf hin, dass dabei vor allem das Experiencing-Niveau bei der Bearbeitung problematischer Kernpunkte von Interesse ist, und dass sich eine $\mathrm{Zu}-$ nahme des Experiencing-Niveaus bei dieser Bearbeitung als starker Prädiktor für den Therapieerfolg herauskristallisierte, während Messungen zu Beginn, am Ende und zu einem $\mathrm{Zu}-$ fallszeitpunkt im Verlauf der Therapie keine Aussage über den Therapieerfolg zuließen. Nach Watson et al (2010, S. 150) sind Focusing-Prozesse auf den Stufen 4 bis 7 der ExperiencingSkala anzusiedeln. 
Kritisch ist anzumerken, dass neben der Experiencing-Skala kein valides Instrument zum Erfassen experienzieller Prozesse etabliert ist. Dies ist sicher auch darauf zurückzuführen, dass Gendlins originäre Konzeption zwar philosophisch fundiert, psychologisch aber nicht klar definiert ist, so dass weitere Aspekte des Experiencing wie beispielsweise die Symbolisierung oder der Felt Shift nur schwer operationalisierbar sind.

Speziell auf Focusing bezogene Übersichtsstudien wurden unter anderem von Jaison \& Lawlor (1996), Hendricks (2002), Purton (2004) und Elliot et al (2013) durchgeführt. Watson et al (2010, S. 150) fassen die Erkenntnisse dieser Studien wie folgt zusammen:

- Eine Reihe von Studien beschreiben bei erfolgreichen Therapien mehr FocusingElemente als bei nicht-erfolgreichen Therapie-Verläufen.

- In einem Großteil der positiv verlaufenden Therapiesitzungen ist ein intensives Experiencing der Klienten zu beobachten, bei dem sie Aspekte ihres Themas oder Problems erkennen, die zuvor noch nicht im Bewusstsein waren.

- Bei diesen Prozessen kommen die Klienten in Kontakt mit ihrem körperlich spürbaren Erleben, ohne allerdings von diesem überwältigt zu werden.

- Focusing ist nicht für alle Klienten indiziert, es gibt auch andere zielführende Psychotherapieprozesse.

Entgegen der anfänglich von Gendlin formulierten Annahme, dass Focusing ein für alle Klienten gleichermaßen geeignetes Verfahren darstelle, zeigen weitere Untersuchungen, dass bestimmte Klientenmerkmale Einfluss auf das Gelingen eines Focusing haben (Sachse et al., 1992, S. 22): Die Explizierung eines Felt Sense scheint eher den Klienten zu gelingen, die über ein durchschnittliches oder hohes Ausmaß an Selbstexplorationsfähigkeit im Sinn der Fähigkeit zur Auseinandersetzung mit sich selbst und dem eigenen inneren Erleben verfügen. Umgekehrt können Abwehr, Widerstand oder Angst gegenüber der Beschäftigung mit bestimmten Inhalten zu aversiven Gefühlszuständen führen, die einen erfolgreichen Focusingprozess verhindern oder beeinträchtigen. Ähnliches gilt für Menschen mit psychosomatischen Beschwerden: auch hier zeigt sich ein im Vergleich zu einer Normalpopulation weniger erfolgreicher Verlauf des Focusingprozesses.

Timulak \& Creaner (2010) fassen als Ergebnis ihrer qualitativen Metaanalyse die Wirkungen personzentrierter und experienzieller Psychotherapien in drei Hauptkategorien zusammen:

- Klienten machen neue Erfahrungen über sich selbst und ihre Emotionen und schätzen diese Erfahrungen. Dazu gehören Aspekte wie Selbstakzeptanz, Mitgefühl mit sich selbst, Resilienz, Empowerment etc.

- Klienten schätzen die Erfahrungen, die sie in der Beziehung mit anderen Menschen machen, sie erleben sich unterstützt.

- Klienten ändern ihre Sicht auf sich selbst und andere (ebd., S. 75).

Die Studie berichtet auch über negative Effekte wie fehlende Lösungsorientierung, überwältigendes Problemerleben oder ein Erschrecken angesichts emotional belastender Themen in 
den Sitzungen. Die Autoren (ebd., S. 83) leiten daraus die Notwendigkeit ab, zwischen produktiven und unproduktiven emotionalen Erfahrungen zu unterscheiden und auf einen vorwärtstragenden emotionalen Prozess zu achten.

\subsection{Kritische Würdigung des Experiencing-Konzepts}

Eine grundlegende Problematik bei der Würdigung von Gendlins Konzept ergibt sich daraus, dass er die psychologisch-therapeutische Relevanz des Konzepts hervorhebt und gleichzeitig darauf verzichtet, eine psychologisch stringente Theorie zu entwickeln. Insbesondere das von ihm postulierte Experiencing-Verständnis steht im Widerspruch zu wesentlichen Erkenntnissen der Kognitionspsychologie und der Entwicklungspsychologie (Sachse et al., 1992, S. 15), so dass eine theoretische Klärung dringend geboten scheint.

Im Sinn einer Würdigung des gendlinschen Ansatzes sei zunächst darauf hingewiesen, dass zum Verständnis des Experiencing-Konzepts auch dessen philosophische Wurzeln herangezogen werden müssen. Hier ist insbesondere das Prozessmodell von Veränderung und Entwicklung von Bedeutung, das Gendlin (2015) auf der der Grundlage existenzphilosophischer und phänomenologischer Überlegungen entwickelte.

Ausgangspunkt des Prozessmodells ist zunächst die Annahme, dass Körper und Umwelt ein prozesshaft vor sich gehendes Ganzes sind. Das Individuum nimmt demnach die Umwelt nicht in erster Linie als etwas Statisches und Beobachtbares wahr, sondern als ein sich dynamisch fortsetzendes Geschehen, bei dem sich Körper und Umwelt gegenseitig implizieren.

„Ein Beispiel: Im Prozess des Gehens sind der Widerstand des Bodens und der Druck des Fußes Aspekte des selben sich dynamisch fortsetzenden Geschehens. (...) Umwelt ist im Prozessdenken zugleich Körper und umgekehrt ist Körper zugleich Umwelt. Dies wird deutlich, wenn man sich vergegenwärtigt, dass im Prozess des Gehens der Widerstand des Bodens bis in den Bau der Knochen im Fuß und bis in die Gelenke und Unterschenkelknochen und in den gesamten Körperbau hineinreicht.“ (Hofmann, 2016, S. 218)

Der Begriff Prozess soll in diesem Modell also den dynamischen und veränderbaren Charakter des Seins einschließlich der darin enthaltenen Interaktionen und Wechselwirkungen beschreiben. In der Kommunikation zeigt sich dieser Prozess dann in Form des „Implizierensins-Geschehen“ und des „Geschehens-ins-Implizieren-hinein“ (Gendlin, 2015, S. 57). Gemeint ist damit, dass jede Bedeutung, die in einem Geschehen impliziert wird, wiederum eine eigene Fortsetzung, ein eigenes Geschehen mit sich bringt. Dieses Geschehen ist allerdings nicht kausal zu verstehen, sondern als ein Forttragen des Prozesses, denn indem etwas geschieht, ist das, was von dort an impliziert wird, etwas anderes. So ist das Implizieren nicht etwas Alleinstehendes, sondern kann nur in Kombination mit aktuellem Geschehen verstanden werden. Zum vorantragenden Prozess wird dieses dann durch Explikation:

„Das erste Gesetz der Explikation besagt, dass eine Explikation mehr und anderes 
umfasst als das Geschehen, in dem das jetzt Explizierte noch implizit war." (ebd., S. 297).

Andererseits besteht auch die Möglichkeit, dass etwas vom Prozess Impliziertes nicht geschieht, Geschehen also gestoppt wird. Dies ist dem Modell zufolge dann der Fall, wenn ein Mangel auftritt, indem der Organismus etwas (ein „Objekt“) vermisst (ebd., S. 67). Dieses Objekt ist nicht ein von Beginn an abgrenzbares oder gegebenes Etwas, sondern es wird dadurch zum Objekt, dass es im Rahmen des Prozessgeschehens als etwas Fehlendes impliziert wird.

Damit der so angehaltene Prozess fortgeführt werden kann, muss das Objekt (wieder) auftauchen, entweder indem der Prozess etwas Neues impliziert, seinen Charakter verändert, oder indem etwas bisher nicht Impliziertes in den Prozess hinein geschieht. So entsteht eine Bewegung, die Neues, Schöpferisches hervorbringt, was aus dem bisherigen Prozess heraus noch nicht prognostizierbar gewesen ist.

Ein weiteres Element des Prozessmodells ist die Vorstellung von drei Räumen, die sich aus gestoppten Prozessen heraus ausbilden können:

1. Der Verhaltensraum, der organismisch ausgebildet wird, um gestoppte Prozesse intuitiv durch Verhalten fortzusetzen.

2. Der symbolische Raum, der sich ergibt, indem Sprache und Symbolik neue Möglichkeiten eröffnen, bisher im Verhaltensraum wahrgenommene Grenzen zu überwinden. Eine Symbolik kann ganz konkret sein, z.B. das Heben der Hand als eine Handlung, um ein Taxi zu rufen. Symbolik kann aber auch durch Repräsentation entstehen, indem eine Symbolisierung für ein Objekt gefunden wird und dann eine Auseinandersetzung mit der Symbolisierung stattfindet.

3. Ein großer, offener Raum: Wenn im symbolischen Raum Stopps in Sinne von angehaltenem Geschehen auftreten, wird der dritte Raum bedeutsam. Hier kommt das zum Tragen, was im Focusing Felt Sense genannt wird: die Symbolisierung verbindet sich mit einem Gespür, mit einem Fühlen. Und während dieses Fühlen sich bildet, „versteht es sich sozusagen selber. Es bringt sein eigenes ,ja, ja, genau..." mit sich“ (Gendlin, 2015, S. 399). Somit entsteht ein Raum, der über das Symbolische hinausträgt bzw. dem Symbolischen neue Möglichkeiten eröffnet und damit voranträgt und neue Handlungen ermöglicht.

Zusammenfassend ist ein Prozess nach diesem Verständnis also gekennzeichnet durch ein Sich-Ereignen in das Implizite hinein, das dieses weiterträgt und ein neues Ereignis ermöglicht. In Gendlins philosophischer Sicht spielen dabei der Körper und die Zeit insofern eine bedeutende Rolle, als dass in seinem Prozessmodell die übliche cartesianische Raum-ZeitSichtweise, die den Raum als dreidimensionales Koordinatensystem und die Zeit als vierte, linear ablaufende Dimension betrachtet, erweitert. Aus dem Prozessdenken heraus ist der Körper nicht statische Materie, sondern er ist lebendig, er ist selbst Prozess. Als solcher trägt 
er seine eigene Vergangenheit in sich, die aber gleichzeitig als solche wirkt und bereits verändert ist durch das, worin sie in der Gegenwart fungiert (ebd., S. 103). Vergangenheit ist demnach also das aktuelle Implizieren der Körperprozesse, die in der Interaktion mit dem gegenwärtigen Geschehen wirksam werden. Als Konsequenz dieser Sichtweise kann der Organismus im Gegensatz zu statischen Objekten nicht stillstehen, sondern wird sich beständig weiterentwickeln, sich regenerieren, sich verändern.

Allerdings findet dieses im Prozessmodell enthaltene In-sich-Tragen der Vergangenheit und ihre Interaktion mit dem Gegenwärtigen in Gendlins psychologischen Ausführungen zum Experiencing nur partiell Berücksichtigung. Es bleibt unklar, welche Rolle vergangene Erfahrungen für das gegenwärtige Erleben spielen. Einerseits betont Gendlin, dass jede gegenwärtige Erfahrung auch vergangene Erfahrungen beinhaltet, so dass Vergangenheit und Gegenwart sich zu etwas Neuem verändern (sh. Kapitel 3.2). Andererseits beschreibt er Veränderung als einen freien, nicht strukturgebundenen Interaktionsprozess. Er geht dabei von einem Selbsterleben aus, bei dem Verarbeitungs- und Deutungsstrukturen, die von früheren Erfahrungen und Erlebnissen geprägt sind, keine Rolle spielen (vgl. Sachse et al, 1992, S. 11). Eine Störung tritt demnach dann auf, wenn das Erleben und das Selbsterleben strukturgebunden stattfindet, also entlang alter, verfestigter Verarbeitungsstrukturen.

Dem entgegen argumentiert Kriz (2010, S. 109) im Entwurf einer personzentrierten Systemtheorie in Anlehnung an Piagets kognitionspsychologische Konzepte (Piaget, 1976) und an die Bindungstheorie (Bowlby, 1988), dass grundlegende intrapersonale Verarbeitungsstrukturen, beispielsweise in Form innerer Arbeitsmodelle oder in Form von Schemata, grundlegend für die menschliche Entwicklung sind. Ohne das Vorhandensein kognitiver Strukturen ist eine angemessene Person-Umwelt-Interaktion nicht möglich (Sachse et al. 1992, S. 15).

Ein weiterer problematischer Aspekt bei Gendlins Konzeption ergibt sich aus der Beschreibung der Interaktion zwischen Felt Sense und Symbolisierung. Wenn er davon spricht, mit dem Impliziten zu denken (Gendlin, 2015, S. 398), oder wenn im Focusingprozess ein „Griff“ für den Felt Sense gefunden werden soll, kann das als Metapher verstanden werden. Wenn die Symbolisierung aber Teil des theoretischen Konzepts sein soll, bedarf sie einer konzeptuellen Verortung in der zugrundeliegenden psychologischen Theorie und muss sich auch an anerkannten Konzepten der Emotionspsychologie und der Kognitionspsychologie messen.

Eine entsprechende theoretische Einordnung nehmen Sachse et al (1992, S. 29ff) vor, indem sie den Prozess, der zu einem Felt Sense führt, als Prozess der motivational-affektiven Informationsverarbeitung einordnen und damit auch von der kognitiven Verarbeitung abgrenzen. Sie beziehen sich dabei auf Zajonc (1980), der sich grundlegend mit affektiven und kognitiven Faktoren der Informationsverarbeitung auseinandersetzt und zu dem Ergebnis kommt:

"Affective factors play an important role in the development and maintenance of preferences. The representation of affect can take a variety of forms, including motor responses and somatic reactions. This explains why cognitive methods of preference 
change that are directed at only one form of representation have seldom been effective." (Zajonc \& Hazel, 1982, S. 123)

Als zentrale Grundannahme ihrer Theorie gehen Sachse et al (1992, S. 33) von einer ,teilweisen Unabhängigkeit“ kognitiver und affektiver Verarbeitung aus. Gemeint ist damit, dass affektive Verarbeitung zwar nicht generell unabhängig von der kognitiven Verarbeitung stattfinden muss, dass sie aber durchaus unabhängig verlaufen kann. Die Autoren kommen zu dem Ergebnis, dass die unabhängige Verarbeitung affektiver und kognitiver Elemente insbesondere bei persönlichen Problemen häufig stattfindet und erklären so, dass Klienten affektive Reaktionen haben können, die sich dem rationalen Verstehen entziehen. Für das affektive Verarbeitungssystem wiederum wird - analog zum kognitiven Verarbeitungssystem - angenommen, dass es einer bestimmten Verarbeitungsdisposition im Sinn affektiver Schemata folgt. Die Verarbeitung durch solche Schemata kann automatisch und unbewusst funktionieren, wobei die Person die Effekte der Verarbeitung spürt, z.B. in Form von Unbehagen, Anspannung oder inneren Impulsen:

„Die affektiven Verarbeitungen (...) führen zu Affekten: Diese sind noch keine Emotionen im engeren Sinne, gehen aber als affektive Bewertungen in die Emotionsgenese ein. (...) Affekte sind sehr unterschiedlich und variabel: Es kann sich dabei um körperlich gut spürbare Reaktionen handeln wie Verspannung im Nacken, Druck im Bauch, Druck auf der Brust, aber auch um „Stimmungen“, wie dem „Gefühl des diffusen Unbehagens“, dem Gefühl, ,irgendetwas stimmt nicht“ etc. (...) Diese Affekte sind nicht einfach körperliche Reaktionen, sondern sie sind hoch informative Signale: Sie signalisieren das Ergebnis affektiver Verarbeitungsprozesse und enthalten damit affektive Bedeutungen. (...) Gendlin nennt diese Affekte „felt senses“ (,gefühlte Sinne“ oder „gefühlte Bedeutungen“).“ (Sachse \& Fasbender, 2011, S. 132)

Inwieweit ein Stimulus (eine Situation, ein Objekt) affektiv verarbeitet wird oder nicht, unterliegt der individuellen Qualität, die das Individuum dem Stimulus zuschreibt. Es ist nicht der Stimulus oder das Objekt, das eine emotionsauslösende Eigenschaft hat, sondern es ist die Wahrnehmung der Person, die dem Objekt diese Eigenschaft verleiht. Offen bleibt dabei allerdings, inwieweit diese „Eigenschaftsverleihung“ aufgrund eines kognitiven Bewertungsprozesses stattfindet, wie ihn Lazarus \& Launier (1981) in ihrem transaktionalen Stressmodell beschreiben, oder inwieweit sie im Rahmen eines affektiven Schemas vorgenommen wird. Aufgabe des motivational-affektiven Verarbeitungsmodus ist, sicherzustellen, „dass das Wohlergehen der Person gewahrt bleibt, er ist somit nicht für die Interaktion mit der Realität, sondern für die „Motivkompatibilität“ von Handlungsergebnissen zuständig.“ (Sachse \& Fasbender, 2011, S. 134)

Die Autoren (ebd.) gehen davon aus, dass affektive Verarbeitungsprozesse bereits früh in der Biographie beginnen und die entsprechenden Erfahrungen und Interpretationen in Form af- 
fektiver Schemata gespeichert werden. Später können sie durch Situationskomponenten wieder aktiviert werden, die den Situationen bei ihrer Entstehung ähnlich sind. Je nachdem, ob die Erfahrungen positiv oder negativ waren, führen die Schemata dann in der aktuellen Situation zu funktionalen oder dysfunktionalen Affekten.

Eine weitere Rolle spielen nach dieser Theorie die in die Zukunft gerichteten Handlungsimpulse und Wunschvorstellungen einer Person, die wiederum auf ihre persönlichen Ziele und Motive zurückgehen. Diese Ziele und Motive fungieren quasi als persönlich-idiosynkratische Wertungsdisposition und bestimmen so mit, welche Ereignisse für die Person positiv oder aversiv sind.

Während kognitive Schemata auch kognitiv repräsentiert sind und damit über Sprache expliziert und bearbeitet werden können, liegen affektive Schemata zunächst nicht oder nur teilweise in einer kognitiven Repräsentation vor. Ziel eines therapeutischen Prozesses ist daher, affektive Inhalte sprachlich auszudrücken, um sie zu verstehen und über sie kommunizieren bzw. sie bearbeiten zu können. Sachse \& Fasbender (ebd., S. 135) beschreiben dazu einen „Übersetzungsprozess“, um zu einer kognitiven Abbildung affektiver Schemata zu gelangen. Um einen Bezug zu den jeweiligen Focusing-Elementen herzustellen, soll der Prozess hier tabellarisch dargestellt und kommentiert werden:

\begin{tabular}{|l|l|}
\hline Übersetzungsprozess affektiver Schemata & Vorgehen im Focusing \\
\hline $\begin{array}{l}\text { Das affektive Schema aktivieren und die } \\
\text { ganze Zeit über aktiviert halten, damit af- } \\
\text { fektive Bedeutungen spürbar sind. }\end{array}$ & $\begin{array}{l}\text { Innerlich Raum schaffen, die Aufmerksamkeit } \\
\text { auf das aktuelle Erleben und die vage körperli- } \\
\text { che Empfindung richten, bei ihr verweilen. }\end{array}$ \\
\hline $\begin{array}{l}\text { Die affektiven Bedeutungen im Fokus der } \\
\text { Aufmerksamkeit halten und sie als Ver- } \\
\text { gleichsmaßstab für gefundene kognitive }\end{array}$ & $\begin{array}{l}\text { Den Felt Sense entstehen lassen als etwas, das } \\
\text { noch nicht in Worte gefasst werden kann, aber } \\
\text { offensichtlich eine Bedeutung hat. }\end{array}$ \\
\begin{tabular}{l} 
Bedeutungen nutzen. \\
\hline $\begin{array}{l}\text { Eine intuitiv-holistische Suche nach pas- } \\
\text { senden kognitiven Elementen starten, die } \\
\text { ähnliche Bedeutungen aufweisen wie die } \\
\text { gespürten affektiven Bedeutungen. }\end{array}$
\end{tabular} & $\begin{array}{l}\text { Einen ,Griff“ finden, eine Symbolisierung, die } \\
\text { dem Ausdruck verleiht, was schwer auszudrü- } \\
\text { cken ist. }\end{array}$ \\
\hline $\begin{array}{l}\text { Gefundene kognitive Elemente mit den } \\
\text { gespürten affektiven Bedeutungen ver- } \\
\text { gleichen und auf ihre Passung hin untersu- } \\
\text { chen. Gegebenenfalls weitere kognitive }\end{array}$ & $\begin{array}{l}\text { Die Stimmigkeit zwischen dem Griff und dem } \\
\text { Elemente suchen, so dass die affektive Be- } \\
\text { noch besser passendes Wort oder Bild finden. }\end{array}$ \\
$\begin{array}{l}\text { deutung möglichst gut kognitiv abgebildet } \\
\text { werden kann. }\end{array}$ & \\
\hline \begin{tabular}{l} 
Tabelle 1: Gegenüberstellung: Übersetzungsprozess affektiver Schemata und Vorgehen im Focusing \\
\hline
\end{tabular}
\end{tabular}


Ein Ergebnis einer derart gestalteten kognitiven Repräsentation des affektiven Schemas ist in der Regel das unmittelbare Verstehen des Schemas. Diesen Moment bezeichnet Gendlin als Felt Shift. Ein weiterer Effekt ist, dass durch die Repräsentation affektiver Schemata auch eine Aktivierung der Affekte eintritt und dadurch in aller Regel auch (positive oder negative) Emotionen ausgelöst werden, die dann weiter bearbeitet werden können. 


\section{Diskurs}

Wie bereits deutlich wurde, ist das Experiencing-Konzept mit einer theoretischen Herausforderung verbunden, die im Wesentlichen darauf zurückzuführen ist, dass Gendlin den Begriff des Felt Sense zwar philosophisch erklärt, psychologisch aber nicht klar definiert und daher eine Einordnung und empirische Überprüfung des Modells erschwert.

Eine Möglichkeit zum Umgang mit dieser Gegebenheit besteht im Diskurs, um den Experienziellen Ansatz in Bezug zu anderen Konzepten und Forschungserkenntnissen zu setzen bzw. von diesen abzugrenzen. Das entspricht auch dem, was Gendlin als „kreuzen“ bezeichnet, als Dialog zwischen dem bisher Verstandenen und anderen Einsichten, der Neues hervorbringen kann:

„Wenn die Bedeutung (...) einmal artikuliert und genügend differenziert worden ist, dann geschieht etwas, was wir „kreuzen“ (crossing) nennen. Einsichten anderer Leute reichern unsere an, indem sie in unseren eigenen Begriffen implizit werden.“ (Gendlin, 2004; Übersetzung H. Mühletaler)

In Rahmen einer kritischen Würdigung des Experiencing-Konzepts wurde bereits der von Sachse et al (1992) gewählte Weg dargestellt, in enger Anlehnung an Gendlin eine psychologische Theorie der affektiven Informationsverarbeitung zu entwerfen, die den Prozess, der zu einem Felt Sense führt, analog zum Prozess der kognitiven Informationsverarbeitung erklärt. Einen anderen Weg, der noch darzustellen sein wird, wählte Greenberg (2006), der auf der Basis des personzentrierten und focusingorientierten Konzepts einen eigenen Ansatz der Prozess-Experienziellen Emotions-Fokussierten Therapie entwickelte, in dem er sich von Gendlins Konzeptionierung des Experiencing als grundlegenden Prozess löst und stattdessen Emotionen als das Grundlegendere betrachtet. Daneben sollen in diesem Kapitel auch Einsichten anderer Orientierungen und weitere coachingspezifische Aspekte des Personzentrierten und des Experienziellen Ansatzes in den Diskurs einbezogen werden.

\subsection{Personzentrierung und Coaching}

Nach De Haan \& Mannhardt (2014) ist eine grundlegende Annahme des Coaching die, dass Menschen als Expertinnen ihrer selbst agieren und auch so verstanden werden wollen. Diese Sichtweise deckt sich mit den Grundüberzeugungen der humanistischen Psychologie und dem Verständnis des Personzentrierten Ansatzes, nach dem die Klientin stets als Expertin ihrer selbst betrachtet wird (Eberwein, 2009, S. 69).

So ist die Haltung, mit der die Coach der Coachee begegnet, als zentraler Aspekt der personzentrierten Beratung zu sehen. Merkmale dieser Haltung sind nach Steinhage (2005, S. 175): 
- Interesse an der Person und deren Anliegen

- Ernstnehmen und verstehen wollen der Coachee und ihres Anliegens

- Exploration des Unklaren und noch nicht Verstandenen

- Unterstützung bei der Bewusstwerdung und Aktivierung der Ressourcen der Coachee.

Durch ihre Haltung stößt die Coach einen Prozess an, der das Vermögen zur Selbsterkenntnis bzw. das „latent Gewusste“ fördert (Seewald, 2006, S. 69).

Die im Personzentrierten Ansatz postulierte besondere Bedeutung der Beziehung zwischen Therapeutin und Patientin bzw. Coach und Klientin geht einher mit den bereits dargestellten für das Coaching relevanten Wirkfaktoren Wertschätzung und Unterstützung. Gessnitzer \& Kauffeld (2018, S. 50) zeigen in ihrer Übersicht zur theoretischen Fundierung von Coaching, dass die von Rogers postulierten Basisbedingungen zur Personzentrierten Beratung (vgl. Kapitel 3.1) im Kontext von Coaching häufig Erwähnung finden, dort jedoch oft mit anderen Begriffen verbunden werden. So werde Wertschätzung als Vertrauen oder als Akzeptanz ohne Vorbedingungen bezeichnet. Auch Empathie werde als Faktor zum funktionierenden und vertrauensvollen Beziehungsaufbau häufig als essenziell dargestellt, insbesondere im Zusammenhang mit der Akzeptanz der Wirklichkeit und dem Eingehen auf die Gefühle und Emotionen der Coachee. Kongruenz werde häufig verstanden als die Wahrung der eigenen Autonomie, die die Coach befähigt, authentisch zu sein und nur diejenigen Aspekte in den Prozess einzubringen, die auch der eigenen Wahrnehmung und Haltung entsprechen.

Eine Untersuchung von Kunze (2016, S. 21) weist darauf hin, dass die allgemein wahr-genommene gegenseitige Wertschätzung zwischen Coach und Coachee zunächst keinen Effekt auf den Coachingprozess hat. Erst die für die Coachee erlebbar gemachte und sich von persönlicher Zuneigung unterscheidende bedingungsfreie positive Beachtung, verbunden mit einem präzisen Einfühlen und Verstehen, bringt dann eine konstruktive Unterstützung der Veränderungsprozesse. Will, Gessnitzer \& Kauffeld (2016) betonen dazu, dass Coachs sich selbst tendenziell empathischer wahrnehmen, als sie von ihren Klientinnen wahrgenommen werden. So führe allein das Verbalisieren emotionaler Erlebensinhalte bei der Coachee noch nicht zu einer Wahrnehmung des Verstandenwerdens, erst das Paraphrasieren der Coachee-Aussagen bewirke diesen Effekt.

Sander \& Ziebertz (2010, S. 69) sprechen in diesem Zusammenhang von einem personzentrierten Beziehungskonzept. Es geht dabei um ein Beziehungsangebot, das der Klientin eine schrittweise Selbstöffnung ermöglicht und in deren Folge auch eine Selbstexploration im Sinn einer aktiven und klärenden Auseinandersetzung mit der Bedeutung ihres inneren Erlebens (Sander \& Ziebertz, 2010, S. 86). Ein weiteres Resultat der im Coaching stattfindenden Selbstexploration und Selbstaktualisierung ist die Aktivierung eigener Ressourcen im Sinn eines selbstständigen Entwickelns von Handlungs- und Lösungsstrategien:

„Wirksame Beratung besteht aus einer eindeutig strukturierten, gewährenden Bezie- 
hung, die dem Klienten ermöglicht, zu einem Verständnis seiner selbst in einem Ausmaß zu gelangen, das ihn befähigt, aufgrund dieser neuen Orientierung positive Schritte zu unternehmen.“ (Rogers, 1972, S. 28)

Aus personzentrierter und experienzieller Sicht differenziert zu betrachten ist der in Kapitel 2.2.1 beschriebene Wirksamkeitsfaktor Zielklärung bzw. Zielformulierung, verbunden mit einer wirksamen Arbeitsallianz, die zunächst scheinbar der im Personzentrierten Ansatz oft geforderten Non-Direktivität und Prozessorientierung der Beraterin (vgl. Rogers, 1972; Wiltschko, 2017) entgegensteht. Mit Blick auf die Aktualisierungstendenz und die von Gendlin postulierte vorwärtstragende Gerichtetheit des Prozesses sind Zielorientierung und Prozessorientierung allerdings nicht als sich gegenseitig ausschließend zu betrachten: jeder in der Person stattfindende Prozess hat eine Richtung und strebt auch nach einer Lösung, die der Erhaltung oder Förderung des Organismus dient (Wiltschko, 2017, S. 141). Die einzelnen Prozessschritte sind dabei von außen eher nicht antizipierbar und nehmen oft einen überraschenden Weg. Vor diesem Hintergrund sind die Ziele und Schritte zum Ziel von der Coachee selbst zu entwickeln und im Prozess anzupassen. Anschaulich verdeutlicht diese differenzierte Betrachtung der Zielklärung eine Studie von Gessnitzer \& Kauffeld (2015) über die Beziehung zwischen Coach und Coachee:

„Interessanterweise machte es jedoch einen großen Unterschied, ob die Ziele und Lösungen vom Coach oder vom Klienten / von der Klientin benannt wurden: Nur wenn der Klient / die Klientin die Ziele und Lösungen formulierte und der Coach zustimmte, wirkte sich dies positiv auf die Zielerreichung im Coaching aus. Waren die Rollen vertauscht, wenn also der Coach die Formulierung übernahm und der Klient daraufhin zustimmte, zeigte sich sogar ein negativer Effekt auf die Zielerreichung.“ (Gessnitzer, Schulte \& Kauffeld, 2018, S. 40)

Folgt man den Analysen von Hofmann (2016, S. 19ff) zur Postmoderne, erfordert die zu beobachtende Komplexität und Radikalität vieler Themen eine Art der Kommunikation, die verbunden ist mit Autonomie und dem Vertrauen in die Entwicklungskräfte des Organismus, und die auch eine bestimmte - personzentrierte - Art der Beziehungsgestaltung beinhaltet. In einer Gegenüberstellung grenzt er (Hofmann, 2016, S. 392) diese Art der Kommunikation ab von einem funktionalen Interagieren und Kommunizieren:

\begin{tabular}{|l|l|}
\hline In-Beziehung-Sein & In-Funktion-Sein \\
\hline $\begin{array}{l}\text { Kontakt; erspüren, was sich von Mo- } \\
\text { ment zu Moment stimmig anfühlt }\end{array}$ & $\begin{array}{l}\text { Abstrakte Regeln, die vorgeben, was } \\
\text { getan werden soll / darf, und was } \\
\text { nicht }\end{array}$ \\
\hline $\begin{array}{l}\text { Vertrauen in die Fortsetzungsordnung } \\
\text { gibt Sicherheit }\end{array}$ & $\begin{array}{l}\text { Sicherheit durch vorab festgelegte } \\
\text { Rechte und Pflichten }\end{array}$ \\
\hline Es sind immer nur die jeweils nächsten & Es kann ein langfristiger (Ablauf-) \\
\hline
\end{tabular}




\begin{tabular}{|l|l|}
\hline $\begin{array}{l}\text { kleinen Schritte sichtbar (implizite } \\
\text { Ziele) }\end{array}$ & $\begin{array}{l}\text { Plan gemacht werden (explizit formu- } \\
\text { lierte Ziele) }\end{array}$ \\
\hline Hohe Verletzbarkeit & Niedrige(re) Verletzbarkeit \\
\hline $\begin{array}{l}\text { Viel Gespür für die eigene Befindlich- } \\
\text { keit notwendig (,sich tagtäglich immer } \\
\text { wieder selbst erforschen“) }\end{array}$ & $\begin{array}{l}\text { Wenig(er) Gespür für die eigene Be- } \\
\text { findlichkeit notwendig (,Regelkennt- } \\
\text { nis“ ist wichtiger als Improvisation } \\
\text { und Intuition) }\end{array}$ \\
\hline $\begin{array}{l}\text { Viel Gespür für das Gegenüber not- } \\
\text { wendig (Empathie, Zuhören) }\end{array}$ & $\begin{array}{l}\text { Wenig(er) Gespür für das Gegenüber } \\
\text { notwendig }\end{array}$ \\
\hline Schlicht, unmittelbar & Ausgearbeitet, mittelbar \\
\hline
\end{tabular}

Tabelle 2: Kommunikation: In-Beziehung-Sein versus In-Funktion sein (Hofmann, 2016)

\subsection{Neurowissenschaften und Körperzentrierung}

Dass die Betonung des Körpers im Coaching neuerdings eine besondere Aufmerksamkeit erhält, wird nicht zuletzt mit aktuellen neurowissenschaftlichen Erkenntnissen in Verbindung gebracht, nach denen menschliches Erleben und Verhalten überwiegend durch unbewusste, vegetativ-somatische Prozesse beeinflusst wird. Dadurch wird die lange Zeit auch im Coaching dominierende descartessche Vorstellung des bewussten Ich als oberste Kontrollinstanz von Denken, Planen und Handeln einmal mehr in Frage gestellt (Ryba, 2019, S. 314). Besondere Beachtung findet dabei das von Roth \& Ryba (2016) entwickelte Vier-EbenenModell der Persönlichkeit und Psyche, das unterschiedliche Gehirnebenen beschreibt, die für vier unterschiedliche Funktionen stehen:

1. Untere limbische Ebene: hier sind Mechanismen angesiedelt, die der Lebenserhaltung und der Erfüllung primärer körperlicher Bedürfnisse dienen, und ebenso genetisch oder vorgeburtlich geprägte Persönlichkeitsmerkmale des Temperaments. Die auf der unteren limbischen Ebene stattfindenden Prozesse sind in der Regel unbewusst und von außen quasi nicht veränderbar

2. Mittlere limbische Ebene: hier geht es um Prägungen und Erfahrungen der ersten drei Lebensjahre, meist im Zusammenhang der Interaktion mit der primären Bezugsperson. Diese Erfahrungen prägen sich tief ein und bestimmen die primäre Sozialisation, sind aber in der Regel später nicht erinnerbar, so dass sie ebenfalls zum Bereich des Unbewussten zählen

3. Obere limbische Ebene: die hier vollzogenen Abläufe haben zum Ziel, die primäre Persönlichkeit mit den Erfordernissen des sozialen Zusammenlebens in Einklang zu bringen. Diese sekundäre Sozialisation umfasst Aspekte wie Kooperativität, Rücksichtnahme, Empathie, Kompromissfähigkeit, Zielstrebigkeit, Selbstwirksamkeit, Durchsetzung. Die Prozesse finden bewusst und vorbewusst statt, was 
bedeutet, dass die Inhalte vom Arbeitsgedächtnis in das Langzeitgedächtnis absinken, so dass sie nicht mehr automatisch erinnert werden. Sie werden im Modell als das ,tiefe Vorbewusste“ bezeichnet

4. Kognitiv-sprachliche Ebene: Hier finden Erfahrungs- und Wissenserwerb statt, und ebenso Kommunikation, sachliches Denken, Handlungsplanung. Emotionen sind dieser Ebene nicht zugeordnet, sie werden von den Instanzen der oberen limbischen Ebene hinzugefügt. So kann die kognitiv-sprachliche Ebene von den limbischen Ebenen beeinflusst werden, umgekehrt hat die kognitiv-sprachliche Ebene aber kaum Einfluss auf die limbischen Ebenen und die damit verbundenen emotionalen Zustände (Roth, 2019, S. 300).

Für die drei limbischen Ebenen beschreiben Roth \& Ryba (2016, S. 135) sechs durch Hormone und andere Botenstoffe gesteuerte Grundsysteme, die die persönliche Entwicklung bestimmen:

1. Das Stressverarbeitungssystem, das festlegt, in welchem Maß eine Person mit Problemen und Belastungen umgehen kann

2. Das Selbstberuhigungssystem, das auch die Fähigkeit einschließt, Gefühle bei sich selbst und anderen zu erkennen

3. Das Bindungssystem, das emotionale Gebundenheit und Empathie beinhaltet

4. Das Impulshemmungssystem, in dem die Fähigkeit zur Kontrolle des eigenen Verhaltens, verbunden mit Zielstrebigkeit, Belohnungsaufschub und Kooperativität entwickelt wird

5. Das Motivationssystem, das die unbewussten und bewussten Motive und Ziele ausbildet

6. Das Realitäts- und Risikowahrnehmungssystem zur Wahrnehmung der tatsächlichen Konsequenzen des Verhaltens und einer darauf aufbauenden Handlungsplanung.

Aus diesen Modellvorstellungen lassen sich unterschiedliche Implikationen für das Coaching ableiten (Roth, 2019, S. 304): die untere und die mittlere limbische Ebene bezeichnet Roth als vegetativ-affektiven Sockel, der den unbewussten Rahmen für die weitere Entwicklung der Persönlichkeit bildet. Als eigentliche Einsatzebene des Coaching bezeichnet er die obere limbische Ebene, während die kognitiv-sprachliche Ebene zwar als Vehikel der Interaktion von Bedeutung ist, für sich allein genommen aber im Coaching keine Wirkung erzeugt:

„-- es müssen immer die limbischen Ebenen und damit die Emotionen, die verhaltenswirksamen und die körperbezogenen Anteile angesprochen werden.“(ebd.)

Konkret fordert Roth (ebd., S. 308), Coaching-Interventionen auf drei Ebenen der Persönlichkeit abzustimmen:

1. auf die Ebene der belastenden Empfindungen, Erinnerungen und Vorstellungen

2. auf die Ebene der unangepassten Verhaltensweisen

3. auf die Ebene des Körpers. 
Hinsichtlich der Bearbeitung belastender Empfindungen, Erinnerungen und Vorstellungen sieht er das Risiko einer Verfestigung, wenn diese nicht durch alternative positive Inhalte abgeschwächt werden. Er empfiehlt daher, diese Ebene in einem mental-emotional entspannten Zustand zu bearbeiten, z.B. nach einer vorhergehenden Entspannungsübung, um so eine größere Offenheit für neue Einflüsse entstehen zu lassen. Ein willentliches „Ersetzen“ negativer Vorstellungen durch positive ist dabei wenig zielführend, vielmehr empfiehlt Roth Verfahren, die im Rahmen entspannter Aufmerksamkeit zu affektiv-emotionalen und körperlichen Erlebniszuständen führen, die eine Überlagerung der negativen Erinnerungen durch positive Erinnerungen und Imaginationen ermöglichen.

Bei der Ebene der unangepassten Verhaltensweisen geht es hauptsächlich um dysfunktionale Verhaltensmuster, die im Coaching durchaus verbal expliziert werden können, dadurch aber noch nicht automatisch hinreichend bearbeitet oder beeinflusst sind. Vielmehr sollte auch hier eine prozedural-implizite nichtsprachliche Ebene berücksichtigt werden, um auf unbewusste oder vorbewusste Antriebe, Motive und Strukturen des Denkens, Fühlens und Handelns eingehen zu können. Diese Sichtweise erinnert an Gendlins Störungskonzeption (sh. Kapitel 3.3), in der er von einem strukturgebundenen Selbsterleben spricht, das einen konstruktiven Veränderungsprozess behindern kann.

Die somatische Ebene, bei der es um körperliche Befindlichkeiten und Reaktionen geht, bezieht sich in dieser Konzeption auf die untere limbische Ebene, die die vegetativen Reaktionen des Körpers beeinflusst. Sie wird vor allem bei Somatisierungen von Belastungen relevant.

Nach Roths (2019, S. 311) Überzeugung gibt der Einblick in die neuropsychologischen Abläufe hilfreiche Hinweise zum Einsatz von Coachingmethoden und Coachinginterventionen. Wenn es um persönliche Veränderung oder Entwicklung geht, ist demnach eine rein kognitivsprachliche Bearbeitung quasi wirkungslos, solange sie nicht auch die auf den limbischen Ebenen stattfindenden Prozesse berücksichtigt.

Vor diesem Hintergrund sollte die in Greifs Coaching-Konzept beschriebene zentrale Rolle der Selbstreflexion (sh. Kapitel 2.1) relativiert werden, denn aus neurowissenschaftlicher Sicht nimmt kognitive Selbstreflexion nur sehr begrenzt Einfluss auf die Veränderung von Wahrnehmung, Erleben und Verhalten, solange sie nicht eine Verbindung zu somatisch-vegetativen Prozessen herstellt (Ryba, 2019, S. 314), wie dies beispielsweise im Rahmen der Selbstexploration im Personzentrierten Konzept oder im Rahmen des Experiencing im Experienziellen Konzept vorgesehen ist.

Insgesamt ist eine weitgehende Parallele zum Personzentrierten Ansatz zu erkennen, dessen Konstrukte des organismischen Erlebens und des Selbstkonzepts sowohl unterschiedliche Ebenen der Wahrnehmung und Verarbeitung beschreiben als auch zwischen unbewussten Prägungen und bewusstem gegenwärtigen Erleben unterscheiden. Eine weitere Gemeinsamkeit zwischen Rogers bzw. Gendlins Ansätzen und den Erkenntnissen von Roth und Ryba 
besteht darin, dass Veränderung in beiden Ansätzen so konzipiert ist, dass die „unten liegenden“ (tiefer liegenden, verdeckten, impliziten) Ebenen der Persönlichkeit die weiter „oben liegenden“ (bewussteren) Ebenen beeinflussen und somit nachhaltige Veränderung „von unten nach oben" stattfindet. So schlagen sowohl experienziell ausgerichtete Ansätze als auch die Neurowissenschaften Methoden und Interventionen vor, die körperliches Erleben und Emotionen berücksichtigen und den Nutzen eines Zustands körperlich-emotional entspannter Aufmerksamkeit beim Erarbeiten von Neuem betonen.

Allerdings sollte auch klar sein, dass die epistemologische Grundlage neurobiologischer Forschung eine andere ist als die der humanistischen Psychologie und deswegen die jeweiligen Erkenntnisse nur bedingt zu vergleichen sind. Als zentrales Unterscheidungsmerkmal sei hier die Aktualisierungstendenz als wesentliches Axiom des Personzentrierten Ansatzes genannt. Sie erklärt den Wunsch zur positiven Entwicklung bzw. im Focusing das Vertrauen auf einen vorwärtstragenden Prozess. Die Neurowissenschaft dagegen beschreibt eine solche Prozessrichtung bisher nicht, sondern leistet ihren besonderen Beitrag, indem sie psychologische Prozesse und Zustände biologisch-neurologisch hinterfragt und erklärt.

\subsection{Einsichten anderer Orientierungen}

„Für die Praxis der Psychotherapie gibt es heute viele Orientierungen (...) Jede Orientierung ist mit einem besonderen Geist, einer bestimmten Kultur verbunden (...) Fruchtbare Diskussionen werden zwischen den Praktikern jeder dieser Orientierungen geführt, die nicht stattfinden würden, wenn sie / wir vereinzelte Individuen wären. Daher brauchen wir es, Orientierungen anzugehören und gleichermaßen in der Lage zu sein, unsere Unterschiede und Gemeinsamkeiten wertzuschätzen." (Gendlin, 1996, S. 273)

Was Gendlin für die Psychotherapie formuliert, soll hier zumindest ansatzweise auf das Coaching angewendet werden: Ansätze und Orientierungen, die aktuell eine gewisse Aufmerksamkeit im Coaching erhalten, sollen in Beziehung zu Focusing und Experiencing gesetzt werden. Dabei geht es nicht um eine vollumfängliche Darstellung der jeweiligen Ansätze, sondern um ausgewählte Aspekte, die die Theorie und die Praxis des Experiencing befruchten könnten.

\subsubsection{Körperorientierte Verfahren}

Ryba (2019, S. 319) ordnet das Focusing ein in die wahrnehmungsorientierten körperzentrierten Verfahren, die über Wahrnehmung und innere Achtsamkeit einen Zugang zum Unbewussten bzw. Vorbewussten schaffen, innere affektiv-körperliche Reaktionen im Hier und Jetzt erkunden und daraus Implikationen für weitere Schritte ableiten. Diese Einordnung beruht auf einer Systematik Geuters (2015, S. 65; vgl. Ryba, 2019, S. 320), der vier Strömungen der Körperpsychotherapie unterscheidet:

1. Affektorientierte Therapie, deren Grundstein der Freud-Schüler Wilhelm Reich 
(1897-1957) mit der Entwicklung der Vegetotherapie legte. Daraus entstanden später der bioenergetische Ansatz (Lowen, 2008), der Körperlesen, bioenergetische Übungen und Charakteranalyse miteinander kombiniert und die Technik der biodynamischen Entspannung (Boyesen, 1987), bei der durch Entspannung und Massage unterschwellig unterdrückte emotionale Muster beeinflusst werden sollen.

2. Wahrnehmungsorientierte Therapie, bei der die erfahrungsorientierte Selbsterforschung im Mittelpunkt steht. Hierzu zählen neben dem Focusing unter anderem die Konzentrative Bewegungstherapie (Stolze \& Badura-MacLean, 1984) und die Funktionelle Entspannung (Fuchs, 1974) als leibpädagogische Ansätze des Spürens sensorischer und motorischer Vorgänge und des Wahrnehmens der damit einhergehenden Gefühle, Bilder und Gedanken. Die Hakomi-Methode (Kurtz, 1985, Weiss, Johanson \& Monda, 2019) widmet sich ebenfalls der Wahrnehmung der aktuellen körperlichen Erfahrung. Sie greift dazu auf buddhistische Konzepte zurück, indem sie eine innere Beobachterin einführt, die der Klientin ermöglicht, sich von ihrem aktuellen Erleben innerlich zu distanzieren und es doch gleichzeitig durchzuarbeiten. Beim Gewinnen neuer Einsichten wird Wert darauf gelegt, dass diese „vom ganzen Leib gespürt und als wahr erkannt“" werden können (Weiss, 2013, S. 272).

3. Beziehungsorientierte Therapie: $\mathrm{Zu}$ erwähnen ist hier in erster Linie die Analytische Körperpsychotherapie, bei der der Körper als Medium angesehen wird, um Zugang zu verinnerlichten Beziehungserfahrungen zu erhalten (Geuter, 2015, S. 65).

4. Bewegungsorientierte Therapie, die sich an tanztherapeutischen Ansätzen orientiert und deren Ziel es ist, inneres Erleben in Bewegungen und/oder Sprache auszudrücken und so den Kontakt zu sich selbst und anderen zu verbessern (Baer \& Frick-Baer, 2001).

Tschacher (2006, S. 17) führt den in der Wissenschaft zu beobachtenden Body-Turn zurück auf Forschungen im Kontext künstlicher Intelligenz, bei denen der Zusammenhang zwischen Affekt / Emotion und Kognition ebenso markant wurde wie die Erkenntnis, dass menschliche Intelligenz einen Körper benötigt, um sich entfalten zu können, wobei sicherlich insgesamt die Erkenntnisse der neurowissenschaftlichen Forschung und gesellschaftliche und kulturelle Entwicklungen ebenso bedeutsam sind für die neuerliche Körperorientierung (Geuter, 2015, S. 8). Die Besonderheit der körperorientieren Verfahren liegt darin, dass sie sich nicht nur praktisch, sondern auch theoretisch auf den Körper bzw. das körperliche Erleben des Menschen beziehen. Das Körpererleben wird als grundlegende Quelle des Selbsterlebens genutzt, weil der Mensch in Sinn eines holistischen Menschenbilds als eine Körper-Seele-Geist-Einheit verstanden wird, so dass er sich in seinem Körper und über seinen Körper erlebt (Geuter, 2015, S. 2). 
Nach Damasio (1994) werden sowohl kognitive Entscheidungsprozesse als auch emotionale Wahrnehmungen von Körperempfindungen beeinflusst. Seiner Theorie zufolge werden Handlungsentscheidungen erst dann getroffen, wenn sie in einer als-ob-Schleife kognitiv durchgearbeitet und anhand einer somatischen Rückmeldung als stimmig bewertet werden. Interozeptivität als Sensibilität bzw. Fähigkeit, implizite körperliche Reaktionen wahrzunehmen, ist demnach auch eine relevante Voraussetzung, um Entscheidungs- und Handlungsfähigkeit herzustellen (Geuter, 2015, S. 139).

Allerdings ist auch hier zu konstatieren, dass die Körperpsychotherapie zwar einerseits als eine der großen Grundorientierungen der Psychotherapie bezeichnet wird (Geuter, 2015, S. 2), dass andererseits aber bisher noch

„keine umfassende psychologische Theorie formuliert ist, die den Menschen als sich seelisch-geistig und körperlich selbst erlebendes Wesen zum Gegenstand hat.“ (ebd., S. 3)

Ähnlich wie Gendlin greifen die Autoren der körperorientierten Ansätze nicht nur auf psychologische Erkenntnisse zurück, sondern auch auf philosophische Sichtweisen der Phänomenologie (vgl. Schwarz, 2015, S. 55). Dies zeigt sich auch im Konzept der Leiblichkeit: Der Mensch wird dort als Leib in der Lebenswelt (Petzold, 2006, S. 103) gesehen bzw. als der von innen wahrgenommene subjektiv empfundene Körper, während der Körperbegriff sich auf das von außen Wahrgenommene, Objektivierbare bezieht (Geuter, 2015, S. 21). Dieser Terminologie folgend, würde aus heutiger Sicht auch der Gendlinsche Körperbegriff eher dem des Leibs entsprechen (vgl. Gendlin, 1996, S. 290). Der Begriff des Felt Sense könnte als ein Aspekt des Embodiment im Sinn von Leibphänomenen verstanden werden, bei denen das kognitive System in Bezug zum gesamten Körper steht (Schwarz, 2015, S. 57). Gleichzeitig stellt sich der Felt Sense, wie Gendlin ihn konzipiert, im Vergleich zu einer reinen Körpererfahrung bzw. Leiberfahrung aufgrund des impliziten Informationsgehalts sehr viel komplexer dar, so dass es zu kurz greifen würde, den Felt Sense als reines Leibphänomen zu begreifen.

\subsubsection{Achtsamkeitsbasierte Verfahren}

Im Rahmen der sogenannten 3. Welle der Verhaltenstherapie rückte dort die bis dahin dominierende Orientierung an Konditionierungen und an Kognitionen etwas in den Hintergrund, um emotionsbezogenen Aspekten und achtsamkeitsbasierten Verfahren mehr Raum zu geben (Heidenreich \& Michalak, 2013, S. 15). Das Achtsamkeits-Konzept bezieht sich zunächst allerdings nicht auf eine spezielle psychologische Schule. Es geht zurück auf buddhistischmeditative Übungswege und beschreibt allgemein einen Zustand besonderer Geistesgegenwart. Achtsamkeit wird verstanden als

„Aufmerksamkeitslenkung auf den gegenwärtigen Moment (,,present moment“) und (so gut es geht) ohne eine Bewertung der Erfahrungen (,non-judgemental“). (...) Die 
mit Achtsamkeit einhergehende Geisteshaltung ist durch eine klare und disidentifizierte Wahrnehmung des Stroms von Gedanken, Gefühlen und Handlungsimpulsen gekennzeichnet.“ (Heidenreich, Mundle \& Michalak, 2013, S. 150)

Bekannt wurde das Achtsamkeits-Konzept zunächst durch das Mindfulness-Based Stress Reduction Programm MBSR (Kabat-Zinn, 1990), bei dem es vor allem um das Einüben der drei Kernaspekte der Achtsamkeit geht:

„1. Absichtsvolle, bewusste Wahrnehmung (...) 2. der gegenwärtigen Erfahrung (Gewahrsein) (...) 3. mit einer Haltung der freundlichen Akzeptanz.“ (Meibert, Michalak \& Heidenreich, 2013, S. 167)

Mit Übungen der Achtsamkeit wird das Ziel verfolgt, sich den aktuellen Gefühlen, Gedanken und Körperempfindungen zuzuwenden, und gleichzeitig eine angemessene innere Distanz zu ihnen einzuhalten, so dass eine beobachtende Haltung gegenüber dem eigenen inneren Erleben möglich wird.

In der bei depressiven Störungen oder zur Rückfallprävention im Suchtbereich angewandten Mindfulness-Based Cognitive Therapy MBCT geht es beispielsweise darum, durch kognitivbehaviorale Interventionen negative sprachliche Repräsentationen des gegenwärtigen $\mathrm{Zu}-$ stands zu erkennen und durch Disidentifikation mit den negativen Kognitionen in einen emotional ausgeglichenen Zustand zu gelangen (Michalak \& Heidenreich, 2013, S. 121). Zentrale Wirkungen der MBCT sind die Erhöhung des Mitgefühls mit sich selbst und die Verbesserung der Fähigkeit, Gedanken aus einer metakognitiven Perspektive zu betrachten (Barnhofer \& Williams, 2013, S. 142).

Meibert (2016, S. 18) stellt hinsichtlich des Therapeutinnenverhaltens eine Parallele zwischen dem Achtsamkeitskonzept und dem Personzentrierten Ansatz fest und bezeichnet die Rogers'schen Basisvariablen Echtheit, Wertschätzung und Empathie als ,achtsamkeitsnah“. Zwar ergeben sich daraus keine weiteren theoretischen Implikationen, trotzdem werden Aspekte erkennbar, die ein In-Beziehung-Setzen zwischen Experiencing und Achtsamkeit sinnvoll erscheinen lassen:

- Auch ohne explizite Bezugnahme auf körperliches Erleben zeigt die Achtsamkeitsforschung, dass die bewusste und freundliche Wahrnehmung der gegenwärtigen Erfahrung hilfreich ist, um die Selbstwahrnehmung bei einer gleichzeitigen angemessenen Distanz zu dem aktuellen inneren Erleben zu fördern

- Achtsamkeitsübungen haben eine gewisse Ähnlichkeit mit dem ersten FocusingSchritt des Raum-Schaffens und können auch zum Einstieg in einen FocusingProzess genutzt werden

- Achtsamkeitsprogramme können experiencingorientiert umgesetzt werden, indem die gegenwärtige Erfahrung bewusst genutzt wird, um einen Felt Sense entstehen zu lassen. 


\subsubsection{Psychoanalytische Konzepte}

Auch wenn in den frühen psychoanalytischen Konzepten Freuds noch Bezüge zu körperorientierten Aspekten enthalten sind, werden körperliche Signale in der psychoanalytischen Tradition eher selten für die therapeutische Arbeit genutzt (Geuter 2015, S. 31). Gleichzeitig ist die Landschaft psychoanalytischer Konzepte inzwischen breit aufgefächert, so dass hier auf einige ausgewählte Aspekte eingegangen werden soll.

Neben der Trieblehre (Libido, Destrudo) und dem Strukturmodell (Es, Ich, Überich) ist die Theorie des Unbewussten (Topisches Modell) ein wesentlicher Teil des von Freud beschriebenen und der Psychoanalyse zugrunde liegenden allgemeinen Menschenmodells (Benecke, 2014, S. 110). Dabei werden drei Bereiche unterschieden: das Unbewusste, das Vorbewusste und das Bewusstsein, wobei Bewusstseinsinhalte entscheidend durch unbewusste Prozesse mitbestimmt und gesteuert werden (Ryba, 2018, S. 106). Das Vorhandensein dieser Bereiche wird auch von den Erkenntnissen der Neurobiologie (sh. Kapitel 4.2) gestützt, und die einzelnen Bereiche lassen sich den von Roth \& Ryba (2016) beschriebenen vier Ebenen der Persönlichkeit und Psyche zuordnen. Das Bewusstsein bezieht sich auf die kognitiv-sprachliche Ebene und einen Teil der oberen limbischen Ebene. Das Vorbewusste und Unbewusste ist den drei limbischen Ebenen zuzuordnen.

Übereinstimmend kann für die psychoanalytische und die experienzielle Sichtweise festgestellt werden, dass beide dem Geschehen im Grenzbereich zwischen dem Bewussten und dem Unbewussten einen großen Einfluss zuschreiben. Unterschiede zeichnen sich allerdings dort ab, wo die psychoanalytische Sichtweise das Unbewusste zum Sitz meist sexueller oder aggressiver Triebe, die auf Befriedigung drängen, erklärt, oder die Auffassung vertritt, das Unbewusste sei der Ort des Verdrängten bzw. des dynamisch Unbewussten, das bei einer Aktivierung regelmäßig Abwehr auslöst. Zwar wird die Annahme, dass im therapeutischen psychoanalytischen Prozess Unbewusstes in das Bewusstsein geholt werden kann (Roth \& Ryba, 2016, S. 264), so im Personzentrierten und Experiencing-Ansatz nicht geteilt, wohl aber die Sichtweise, dass es latente Elemente gibt, die nahe daran sind, ins Bewusstsein zu steigen. Vor diesem Hintergrund weist Gendlin (1968, S. 208) auf die Ähnlichkeit seines Konzepts des Impliziten mit den psychoanalytischen Begriffen „,verdrängt“ oder ,unbewusst“" hin. Auch hinsichtlich der Wirkung von psychoanalytischen Deutungen sieht Gendlin Parallelen zur Symbolisierung und dem Felt Shift:

„An experiential effect is also the aim of good psychoanalytic interpretations. (...) Thus the client-centered reflection and the psychoanalytic interpretation are quite similar, when done effectively.“ (ebd., S. 211)

Im psychoanalytischen Strukturmodell steht das Überich oft für eine kritische, innere Stimme, beispielsweise in Form unbewusster Schuldgefühle (Benecke, 2014, S. 119). Den Umgang mit den negativen, Entwicklung hemmenden Facetten des Überichs bezeichnet Gendlin als eine der „Hauptsorgen kognitiver und interpretativer Therapeuten“ (Gendlin, 1996, S. 387). Im Personzentrierten Ansatz wird dieser Aspekt oft mit „,innere Kritikerin“ bezeichnet, die 
dem Individuum wie eine Autoritätsperson mit nicht sachgerechter und destruktiver Einstellung begegnet. Es ist Aufgabe der Therapeutin oder der Coach, dem Überich gegenüber wachsam zu sein und der Klientin die Unterscheidung zwischen dem Felt Sense und der inneren Kritikerin zu ermöglichen. Dies kann geschehen, indem der Prozess immer wieder dorthin zurückgeführt wird, wo die Klientin sich vor dem Auftauchen der inneren Kritikerin befand. Auch ein humorvoller, nicht respektierender Umgang mit der Stimme des Überichs, oder ein bewusstes Nachspüren der Gefühle, die die innere Kritikerin kennzeichnen, kann dazu führen, sie zu erkennen und ihr damit die Macht zu nehmen (ebd., S. 398).

Im Kontext der psychoanalytischen Orientierung ebenfalls zu erwähnen ist das von Fonagy, Gergely, Jurist \& Target (2002) auf Basis der Theory-of-Mind-Forschung entwickelte Mentalisierungskonzept, das in jüngerer Zeit auf auch arbeitsweltliche Felder übertragen wurde (Taubner \& Kotte, 2018, S. 353). Unter Mentalisierung wird die Fähigkeit verstanden, sich mentale Zustände wie Gefühle, Wünsche und deren Zusammenhang mit Verhalten im eigenen Selbst und in anderen Menschen vorstellen zu können (Benecke, 2014, S. 141). Die Mentalisierungstheorie versteht sich als ein Brückenkonzept, das auch die Facetten Achtsamkeit und Empathie einschließt und die Aufmerksamkeit nach innen auf das eigene Erleben einschließlich körperlicher Reaktionen richtet (Taubner \& Kotte, 2018, S. 355). Operationalisiert wird die Mentalisierungsfähigkeit über die Reflective Function, unter anderem mit den Kriterien spezielle Erwähnung mentalen Befindens, Einfühlungsvermögen in die Charakteristika mentalen Befindens und Einfühlungsvermögen in die Komplexität, Unterschiedlichkeit und Vielfalt mentalen Befindens (Benecke, 2014, S. 142), so dass das Konstrukt einige Parallelen zur Experiencing-Skala, auf die später noch eingegangen wird (sh. Kapitel 4.6), aufweist. Als besonders hilfreich für das Coaching werden die im Mentalisierungskonzept beschriebenen unterschiedlichen Modi der Wahrnehmungs- und Affektverarbeitung erachtet, die dem erfolgreichen Mentalisieren entgegenstehen können: teleologischer Modus, Modus der psychischen Äquivalenz und Als-ob-Modus. Im teleologischen Modus steht ein unmittelbarer Handlungs- und Lösungsdruck im Vordergrund, im Modus der psychischen Äquivalenz wird die eigene Befindlichkeit auf die anderen verallgemeinert, und im Als-Ob-Modus werden mentale Befindlichkeiten von der Realität entkoppelt und verlieren damit ihre Bedeutsamkeit (Taubner \& Kotte, 2018, S. 356). Diese Modi werden als entwicklungspsychologische unreif oder auch als regressiv eingeordnet, und die Aufgabe im Coaching ist dann, zu einem reifen Verarbeitungsmodus im Sinn des Mentalisierens zu gelangen. Neben anderen Effekten erleichtert dieser reife Verarbeitungsmodus die für das Experiencing wichtige Unterscheidung zwischen inneren Repräsentanzen und äußerer Realität bei gleichzeitigem Herstellen wesentlicher Bezüge zwischen innerer und äußerer Welt (Weiss, 2011, S. 50).

\subsubsection{Hypnosystemische Beratung}

Der hypnosystemische Ansatz (Schmidt, 2011, 2014) kombiniert systemische Konzepte und die Erickson'sche Hypnotherapie unter Berücksichtigung ausgewählter Aspekte weiterer Ansätze in einem integrierten Modell. 
Das hypnotherapeutische Konzept differenziert zwischen einer bewussten, willkürlichen Erlebnisinstanz und einer Ebene der unwillkürlichen, körperlichen und seelischen Prozesse (Schmidt, 2014, S. 11). Die unwillkürlichen Prozesse werden meist stärker erlebt als die willkürlichen, und bei einer Abweichung der beiden Prozesse entstehen Inkongruenzen, die als Störung empfunden werden. Ziel der Hypnotherapie ist, unbewusste und unwillkürliche Prozesse zu beeinflussen. Der Ansatz wurde von Erickson phänomenologisch anhand zahlreicher Fallvignetten entwickelt, einer umfassenden Theorieentwicklung stand er allerdings skeptisch gegenüber. Er sah darin eher ein Hindernis für die therapeutische Praxis und hatte Sorge, dass die Theorie die unvoreingenommene Wahrnehmung und Nutzbarmachung individueller Verschiedenheiten einschränken könnte (Ryba, 2018, S. 233).

Für den hypnosystemischen Ansatz betont Schmidt (2014, S. 20), dass die traditionell mit Hypnotherapie verbundene Tranceinduktion oft nicht indiziert ist, sondern dass es vielmehr darum geht, im Rahmen einer Konversation einen Prozess der Aufmerksamkeitsfokussierung einzuleiten, in dessen Rahmen sich die gewünschten Prozesse des unwillkürlichen Erlebens einstellen.

„Unwillkürliche Prozesse wirken grundsätzlich immer schneller, effektiver und auch energetisch ökonomischer. (...) Gerade das qualitative Erlebniskriterium „unwillkürlich“ und seine optimale Koordination mit willkürlichen „Ich“-Prozessen (ichsyntones Erleben) erweist sich als von besonderem Interesse für eine erfolgreiche Lebensgestaltung.“ (Schmidt, 2014, S. 23)

Weiter wird im hypnosystemischen Konzept unterschieden zwischen dem expliziten, narrativen Gedächtnis und dem impliziten Gedächtnis (ebd., S. 26). Das explizite Gedächtnis ist eher für kognitive und in Sprache gefasste Interventionen zugänglich. Das implizite Gedächtnis funktioniert zumindest teilweise unbewusst, die dort stattfindenden Prozesse sind meist durch Gefühle und intensive Imaginationsprozesse gekennzeichnet, die sich über unwillkürliche Körpersignale, Empfindungen, Änderung der Atmung, Verspannungen etc. äußern. Da sie neben dem expliziten Gedächtnis ebenso einen wesentlichen Beitrag zur Realitätskonstruktion und damit zur Selbststeuerung des Individuums beitragen, sollten auch sie in der therapeutischen oder beraterischen Arbeit berücksichtigt und nutzbar gemacht werden. Methodisch gelingt das mit Aufmerksamkeitsfokussierung bzw. dem Arbeiten mit Bildern, Metaphern, Imaginationen und rituell sich wiederholenden Bewegungsabläufen, Rhythmik, Tanz (Ryba, 2018, S. 288; Schmidt, 2014, S. 33).

Aus systemtheoretischer Sicht finden sich in Systemen mit den Prinzipien der Auto-poiese und der Selbstreferenz zwei grundlegende Charakteristika, die die Selbstorganisation von Komponenten und von Zuständen eines Systems beschreiben (Hein \& Sewz, 2005, S. 40). Das Phänomen der Autopoiese legt dar, dass die innere Struktur bzw. das innere, Identität schaffende Geschehen eines Systems für dessen Entwicklung von zentraler Bedeutung ist, und ergänzend verdeutlicht das Merkmal der Selbstreferenz, dass Systeme nicht determinis- 
tisch von außen gesteuert werden können, sondern dass externe Einflussnahme nur einen ergebnisoffenen Prozess der Veränderung und Entwicklung anstoßen kann. Diese systemtheoretischen Charakteristika stimmen mit der Aktualisierungstendenz im Personzentrierten Ansatz insofern überein, als dass sie ebenfalls den Optimismus im Blick auf die Selbstentwicklungskräfte und auf die zur Verfügung stehenden Ressourcen des Individuums bzw. des Systems betonen:

„Die synergetische Systemtheorie gestattet, den Menschen aus einer wissenschaftlichen Perspektive so zu sehen, wie Rogers ihn aus seiner menschlichen Perspektive gesehen hat." (Hummitzsch, 2001, S. 4).

Die Störungskonzeption im hypnosystemischen Ansatz geht davon aus, dass die Aufmerksamkeitsfokussierung auf das Implizite bzw. Unwillkürliche nicht nur autopoietische Prozesse hervorbringen kann, sondern dass es ebenso auch eine Art selbsthypnotischer Tranceinduktion geben kann, bei der das Individuum sich als Opfer der unwillkürlichen Prozesse erlebt, ohne dass es ihm gelingen würde, sich in ausreichendem Maß hiervon zu distanzieren. Diese so genannten Muster gilt es dann durch entsprechende Interventionen zu durchbrechen, um innerlich andere Funktionsmuster erlebbar werden zu lassen (ebd., S. 44). Das theoretische Modell geht damit von einem unbewussten Erlebnisrepertoire aus, in dem emotional geladene Erlebnisprozesse jeweils als eigenständige Erlebnisnetzwerke bzw. Episoden gespeichert werden (Schmidt, 2011, S. 20). Sie bleiben zunächst zum größten Teil unbewusst, können aber durch Aufmerksamkeitsfokussierung beschreibbar und damit bewusst erfassbar gemacht werden.

Auch im hypnosystemischen Ansatz spielt das Geschehen zwischen Klientin und Beraterin eine zentrale Rolle, denn die soziale Beziehung wird als essenzielle Rahmenbedingung für Selbstorganisation und Wachstum erachtet (Hüther \& Dohne, 2011, S. 48). Im Unterschied zum personzentrierten und experiencingorientierten Vorgehen übernimmt die Beraterin dabei neben der Steuerung der Aufmerksamkeit die Aufgabe, eigene Ideen und Hypothesen zu entwickeln und aktiv in den Prozess einzubringen (ebd., S. 26). Diese sollen der Klientin ermöglichen, den Horizont der eigenen Wahrnehmung zu erweitern und alternative Muster zu erkennen.

\subsubsection{Prozess-Experienzieller Emotionsfokussierter Ansatz}

Greenberg, Rice \& Elliot (2003, Elliot \& Greenberg, 2007) entwickelten unter dem Namen Process-Experiential / Emotion-Focused Therapy (PE-EFT) einen Therapieansatz und ein Emotionsmodell, das humanistische Konzepte des Personzentrierten und des Experienziellen Ansatzes und der Gestalttherapie verbindet mit Theorien der konstruktiven Informationsverarbeitung. Der Ansatz baut auf auf die Beobachtung, dass sich unter den humanistischen Verfahren gerade diejenigen als besonders effektiv erweisen, die die Klientin über spezifische aktive Interventionen darin fördern, ihr inneres Erleben und insbesondere ihre Emotionen zu 
verbalisieren (Greenberg et al, 2003, S. 24). Das der PE-EFT zugrunde liegende Menschenbild wird von Elliot \& Greenberg (2007, S. 243) als neo-humanistisch bezeichnet und geht davon aus, dass der Mensch selbstbestimmt und weiterentwicklungsorientiert ist, und dass es mehrere innere Teile oder Stimmen gibt, die in einem dialektischen Zusammenspiel mehr ergeben als die Summe ihrer Einzelteile. Affekte und Emotionen spielen dabei eine zentrale Rolle, denn sie sind

„unlöslich mit dem Verhalten, der Bedeutung und der Motivation verknüpft. Affektive Ziele sind so eng mit den Kognitionen verflochten, dass sich die affektiv-motivationalen und die kognitiven Aspekte von Erlebnissen kaum voneinander trennen lassen.“ (Greenberg, 2000, S. 78)

Erleben wird betrachtet als eine sich stetig und dynamisch entwickelnde Synthese emotionaler Schemata, die sich um die jeweiligen Gefühlslagen herum organisieren. Konkret kommen in der Emotionstheorie der PE-EFT zwei Teilkonzepte zum Tragen: emotionale Schemata und unterschiedliche Formen emotionaler Reaktionen.

Als emotionale Schemata werden implizite, idiosynkratische Strukturen des Erlebens bezeichnet (Elliot \& Greenberg, 2007, S. 243). Sie sind dem Bewusstsein nicht unmittelbar zugänglich, sind aber an komplexen selbstorganisierenden Prozessen einschließlich des Bewusstseins, der Handlungen und der Identität, beteiligt.

„Im Unterschied zu kognitiven Schemata bestehen emotionale Schemata zu weiten Teilen aus nonverbalen, affektiven Elementen (Input), wie Körperempfindungen, Handlungsimpulsen, Bildern oder Gerüchen (...) Im Kern sind sie wortlose Geschichten unserer gelebten emotionalen Erfahrung.“ (Auszra, Herrmann \& Greenberg, 2017, S. 27)

Von dem Hintergrund unterschiedlicher emotionaler Erfahrung zeigen Menschen auch unterschiedliche emotionale Reaktionen, und Elliot (2012b, S. 111) beschreibt vier grundlegende Arten dieser Reaktionen:

- primäre adaptive emotionale Prozesse als direkte und nützliche Reaktion auf eine Situation, wie z.B. Ärger als Reaktion auf Verletzung durch andere

- sekundäre reaktive emotionale Prozesse als Reaktion auf eine vorhergehende primäre Emotion. Sie ergänzen die primäre Emotion, wie z.B. Ärger als Reaktion auf primäre Angst oder Traurigkeit

- primäre maladaptive emotionale Prozesse sind ebenfalls als direkte Reaktion zu verstehen, basieren aber auf einem erfahrungsbasierten falschen Verständnis und erscheinen so maladaptiv und dysfunktional

- instrumentelle emotionale Prozesse als nach außen dargestellte emotionale Zustände, um andere Menschen zu beeinflussen. Ein Beispiel dafür sind „Krokodilstränen“ für instrumentelle Traurigkeit.

Ziel therapeutischer Arbeit ist dann, Menschen zu helfen,

„a. ihre (schemagesteuerten) emotionalen Reaktionen bewusst wahrzunehmen, um 
sie flexibel nutzen zu können (Utilisation) und b. Schemata, die nicht länger hilfreich sind, mithilfe von alternativen, adaptiven emotionalen Reaktionen zu verändern (Transformation).“ (Auszra et al., 2017, S. 30)

Dies geschieht durch ein personzentriertes und gleichzeitig den Prozess führendes Therapeutinnenverhalten, von Elliot \& Greenberg (2007, S. 244) als following und guiding bezeichnet. Mit guiding ist gemeint, dass die Therapeutin sich aktiv als Kennerin emotionaler Prozesse einbringt und die Klientin beim Experiencing ,in the manner of Gendlin and others“ (ebd.) anleitet. Neues entsteht dann,

„wenn Klienten lernen, ihr Erleben kontinuierlich aufmerksam zu verfolgen und sich dadurch seiner verschiedenen Aspekte bewusst zu werden, sie zu symbolisieren und sie weiterzuentwickeln.“ (Greenberg et al, 2003, S. 80)

In der Begleitung von Klientinnen kommt damit neben dem Beziehungsaspekt auch dem psychologischen Aufgabenbezug eine wichtige Rolle zu. Elliot \& Greenberg (2007, S. 250) nennen drei wesentliche Aufgaben (Task Principles), die zu berücksichtigen sind:

- Erlebensorientierte Verarbeitung: Ein optimaler, der jeweiligen Situation und dem Erleben der Klientin angepasster Prozess soll gefördert. Dies geschieht, abhängig von den Erfordernissen des jeweiligen Mikro-Prozesses durch aufmerksames Begleiten dessen, was dem Bewusstsein zur Verfügung steht, durch experienzielles Erforschen des inneren Erlebens und die Suche nach Worten für das Unklare, durch Explizieren des inneren Erlebens, durch interpersonellen Kontakt, Selbstreflexion oder Handlungsplanung

- Aufgabenerfüllung/Fokussierung: Die Klientin soll darin unterstützt werden, die zentralen therapeutischen Aufgaben zu lösen. Die Unterstützung besteht darin, der Klientin zu helfen, ihre wichtigsten therapeutischen Aufgaben zu identifizieren und sich darauf zu fokussieren. Benecke (2014, S. 561) weist an dieser Stelle darauf hin, dass die Therapeutin aufgrund ihrer Orientierung am Behandlungsfokus und der Aufgabe des Guiding in der Praxis der PE-EFT teilweise eine stark lenkende Funktion einnimmt, was dem Prinzip der Selbstaktualisierung widerspricht und eher aus psychodynamischen und kognitiven Therapieansätzen bekannt ist

- Selbstentwicklung: Das Wachstum, die Eigenverantwortung und Selbstbestimmung der Klientin soll gefördert werden, indem ihre Handlungsfreiheit (auch innerhalb der Therapie) betont wird und sie durch einfühlsame und motivierende Begleitung ihre persönlichen Wachstumsmöglichkeiten entdeckt.

Im Gegensatz zur Gendlinschen Konzeption wird im Emotionsmodell der PE-EFT das Experiencing nicht als grundlegender Prozess betrachtet. Dies hat zur Folge, dass das Konzept des Felt Sense und der Symbolisierung ebenso in den Hintergrund tritt wie die teilweise schwer zu fassende theoretische Differenzierung zwischen affektiver und emotionaler Verarbeitung bei Sachse et al (1992). Als Verdienst von Greenberg und Kollegen ist anzusehen, dass mit der PE-EFT eine empirisch fundierte Emotionstheorie entwickelt wurde, die inzwischen auch 
von Autoren anderer Verfahren übernommen wurde (Benecke, 2014, S. 193).

Darüber hinaus haben Greenberg und Kollegen mit der Spezifizierung der Task Analyse (Greenberg, 2007, Rice \& Greenberg, 1984, Pascual-Leone, Greenberg \& Pascual-Leone, 2009) einen auch für experiencingorientierte Verfahren interessanten Ansatz der psychologischen Prozessforschung entwickelt, für dessen Verständnis die Kenntnis des PE-EFTEmotionsmodells und der darin enthaltenen Task Princples hilfreich ist und auf den später noch einzugehen sein wird.

\subsection{Varianten des Experiencing}

Neben der Focusing-Methode im engeren Sinn können im Coaching auch Varianten experiencingorientierten Vorgehens von Interesse sein, so dass sie im Rahmen dieses Diskurses ebenfalls berücksichtigt werden sollen.

\subsubsection{Experienzieller Methodeneinsatz}

„Focusing macht (...) alle anderen therapeutischen oder Selbsthilfe-Methoden wirksamer, indem es sie zum körperlichen Felt Sense in Beziehung setzt. (...) Focusing verträgt sich sehr gut mit jeder anderen Methode, die jemandem hilft, und kann diese wirkungsvoll ergänzen. Umgekehrt sind wir jedem dankbar, der uns andere Methoden beibringt. Vielfach herrscht die Meinung, die verschiedenen Methoden würden einander widersprechen, aber im menschlichen Körper kann kein Prozess, der hilft, einem anderen hilfreichen Prozess entgegenstehen."(Gendlin, 1998, S. 179)

Feuerstein \& Müller (1998, S. 43) weisen in ihren Ausführungen zur experienziellen Methode in Supervisionsprozessen darauf hin, dass Focusing-Prozesse als implizite Klärungsprozesse je nach Situation auch ohne explizite schrittweise Focusing-Anleitung stattfinden können. Aufgabe der Prozessbegleitung ist in diesen Fällen, dass die Supervisorin / Coach / Beraterin unabhängig von der jeweils eingesetzten Methode im Gespräch immer wieder zu einer Überprüfung einlädt, ob ein Felt Sense und ein Felt Shift entsteht, konkret: ob ein Gedanke, eine Übung, ein Lösungsansatz mit dem inneren körperlich spürbaren Erleben in Übereinstimmung kommt.

„Mit der Experienziellen Methode soll also nicht inhaltlich festgelegt werden, mit welchen Methoden, Erklärungen etc. wir KlientInnen (...) bei ihrer Veränderung (Lösung, Entwicklung) helfen sollen, sondern wie wir Konzepte (Methoden, Techniken, Wissen...) so gebrauchen in der prozessbegleitenden Beziehung, dass in der Klientenposition der Prozess psychisch-körperlicher Erleichterung als Teil eines persönlichen Entwicklungsprozesses erlebbar wird.“(Feuerstein \& Müller, 1998, S. 44)

Das Interesse beim experienziellen Methodeneinsatz gilt damit primär dem persönlichen Erleben einer Situation oder Fragestellung und weniger der Situation selbst, ihrer Vorgeschichte oder sonstigen Umweltfaktoren. Lösungsansätze entstehen dann nicht extern, sondern durch internal spürbare Prozesse. 
Gendlin (1996, S. 271ff) benennt in seinen Ausführungen zur Integration anderer therapeutischer Methoden zahlreiche Beispiele zum experienziellen Methodeneinsatz, unter anderem:

- Rollenspiel: es gibt unterschiedliche Varianten, um Klientinnen verschiedene Rollen, Positionen, Sichtweisen einnehmen zu lassen, oder auch im Rollenspiel verschiedene Gefühle zu spielen. Der experienzielle Ansatz im Rollenspiel geht dabei weg vom äußeren spontanen Handeln und hin zum inneren, körperlich gespürten Erleben. Wenn es beispielsweise um ein Gefühl geht, steht nicht das äußerliche Ausagieren dieses Gefühls im Vordergrund, sondern das körperlich gespürte Erleben im Zusammenhang mit diesem Gefühl.

- Verhaltenstherapeutische Konzepte: hier empfiehlt Gendlin beispielsweise, neue Handlungen in kleinen Schritten einzuüben, nach Möglichkeit zuerst in sicheren Situationen. Wenn dies gelingt, kann die Person die Wirkung ihrer Handlung auf ihr eigenes Erleben untersuchen. Wenn der Handlungsschritt „stimmig“ im Blick auf das körperliche Erleben war, wird dadurch Energie entstehen, die im Sinn des Prozessmodells eine Weiterentwicklung fördern wird.

- Imaginationen: Imaginationen kommen in verschiedenen Schulen zum Einsatz, entweder in Form einer bildlich-symbolhaften Darstellung einer aktuellen, in der Regel problematischen, Situation, oder in Form einer in die Zukunft gerichteten, in der Regel an einer Lösung oder an einem Wunder orientierten, Projektion. Auch hier geht es im experienziellen Ansatz nicht in erster Linie darum, die Bilder zu betrachten und zu interpretieren, sondern ihre unmittelbare Wirkung auf den Körper einzubeziehen und daraus weitere, am Felt Sense und Felt Shift orientierte Schritte abzuleiten.

Zentral ist dabei die Art des Zuhörens und Begleitens durch die Therapeutin oder die Coach, wie Gendlin (1968) sie in seiner Anleitung zum experienziellen therapeutischen Antworten beschreibt. Hier grenzt er das experienzielle Antwortverhalten auch gegenüber dem rein personzentrierten Antwortverhalten ab: während letzteres primär das erlebte Gefühl im Sinn spezifischer Emotionen wie Liebe, Freude, Ärger etc. reflektiert, bezieht sich die experienzielle Antwort eher auf das komplexere Erleben, auf das Unklare, auf komplexe Person-SituationZusammenhänge und das, was daran im Sinn des Felt Sense körperlich spürbar ist. So wird die Aufmerksamkeit der Klientin auf das Erleben und den Felt Sense gerichtet. Im weiteren Prozess verweilt die Therapeutin / Coach dann bei dem gerade vor sich gehenden Erlebensprozess der Klientin und hilft ihr, diesen zu vertiefen, bis ein Felt Shift, eine Veränderung in der direkten Bezugnahme, meist in Form eines Gefühls der Erleichterung, eintritt.

Experienzielles Antwortverhalten lässt sich wie folgt zusammenfassen:

- „Wir beziehen uns auf den Felt Sense.

- Wir versuchen, den Felt Sense zu explizieren.

- Wir schlagen verschiedene Richtungen vor, um einen erlebensmäßigen Fortschritt zu ermöglichen. 
- Wir folgen der Erlebensspur des Klienten.

- Antworten zielen auf den Felt Sense.

- Wir versuchen, das Erleben fortzusetzen.

- Nur die Person selbst kennt ihre Fährte: Wir lassen uns von ihrer Erlebensspur leiten.

- Nur Veränderung des Bezugspunkts ist Fortschritt.

- Erlebenstiefe geht hinein in das konkret vor sich gehende Erleben." (Gendlin, 1968, S. 219, Übersetzung J. Ossenbach)

Aus den Ausführungen wird bereits deutlich, dass in der therapeutischen Praxis, und analog auch im Coaching, eine Abgrenzung zwischen einem experienziellen Methodeneinsatz nach Gendlin und einem prozess-experienziellen Vorgehen nach Greenberg nur schwer vorzunehmen sein wird. In beiden Richtungen geht es um gespürtes Erleben, und die Differenzierung besteht dann darin, dass im eher focusingorientierten Vorgehen die Aufmerksamkeit bewusst auf eine reagierende Körperstelle gelenkt wird, um dort einen Felt Sense entstehen zu lassen.

\subsubsection{Thinking at the Edge und erlebensorientiertes Concept Coaching}

Betrachtet man Focusing als einen Weg, um dem Impliziten am Rand der Gewahrwerdung Ausdruck zu geben, so kann Thinking at the Edge (TAE) als ein Weg bezeichnet werden, um Neuem, das sich bisher noch schwer in Sprache fassen lässt, Worte zu geben (Gendlin, 2004; Gendlin \& Hendricks, 2004). Feuerstein \& Deloch (2010; Deloch \& Feuerstein, 2013) entwickelten auf Basis von Gendlins TAE-Ansatz das Erlebensbezogene Concept Coaching (ECC), das die Denkschritte des TAE um verschiedene Ebenen der Prozessbegleitung ergänzt. In beiden Ansätzen geht es darum, den Weg zum Neuen über das Unklare und noch nicht Geformte zu finden. Eingesetzt wird es unter anderem zur Entwicklung oder Weiterentwicklung von Konzepten.

TAE beinhaltet 14 systematische Schritte, die dabei unterstützen sollen, das implizite Wissen in einem bestimmten Themengebiet $\mathrm{zu}$ entfalten und daraus stimmige und konsistente Konzeptionen zu entfalten. Die Schritte lassen sich in drei Phasen zusammenfassen (Feuerstein \& Deloch, 2010):

1. Aus dem Erleben der Idee heraus Worte entstehen lassen: Diese Phase leitet dazu an, durch erlebensbezogene Übungen erste stimmige Wörter zu einem Thema zu finden und daraus Sätze zu entwickeln, die das Neue umschreiben und bewahren

2. In beispielhaften Episoden Muster finden: Hier werden Fallbeispiele gesammelt und beschrieben, um einzelne Teile des Konzeptes, die zwar ausgedrückt werden können, aber noch keinen Zusammenhang erkennen lassen, zu konkretisieren. Durch dieses „Kreuzen“ werden wichtige Details und neue Muster herausgearbeitet. „Kreuzen“ ist eine immer wieder zum Einsatz kommende Methode: In unterschiedlichen Episoden wird nach übergreifenden Mustern gesucht, die miteinander verglichen werden mit dem Ziel, daraus weitere, neue Muster zu entdecken 
3. Ein konsistentes Konzept formulieren: Am Ende werden logische Zusammenhänge zwischen den bisher formulierten Teilkonzepten hergestellt, so dass ein kommunizierbares, logisch nachvollziehbares Gesamtkonzept entsteht.

Da der Prozess der Konzeptentwicklung regelmäßig durch blockierende innere Prozesse gekennzeichnet ist, empfehlen Feuerstein \& Deloch (2010), TAE zu ergänzen um Haltungen und Interventionen, die die störenden Prozesse adressieren und damit die Entwicklung von Neuem unterstützen. Gemeint sind damit Aspekte wie:

- Den Prozess schützen vor der inneren Kritikerin. Aufsteigen lassen, was kommt, ohne zu bewerten.

- Auf einen günstigen Abstand zum Thema achten (nicht zu nah, nicht zu fern).

- Umgehen mit wiederkehrenden belastenden Gefühlen.

- Auseinandersetzung mit inneren Reaktionen, die in kritischen Phasen der Konzeptentwicklung vorkommen. Konzepte der Selbstwirksamkeit und Selbststeuerung aufgreifen.

Deloch (2017) hat hierfür einen aus 8 Schritten bestehenden Leitfaden zum Erlebensbezogenen Concept Coaching (ECC) entwickelt, der den Prozess des Erlebens und Entwickelns von neuen Denkspuren veranschaulicht:

1. „Um welches Thema / Anliegen geht es Ihnen?

2. Was daran beschäftigt, interessiert Sie besonders?

3. Was ist das noch schwer sagbare Neue, das Ihnen vorschwebt - welche Veränderungsimpulse, erste, vielleicht noch vage Ideen kommen Ihnen? Etwas, was Sie, ausgehend von Ihrer Erfahrung, irgendwie ahnen oder „wissen“?

4. Mit welcher persönlich von Ihnen gemachten Erfahrung bzw. mit welcher Situation ist dieses Besondere, Neue, um das es Ihnen geht, für Sie verbunden?

5. Wenn Sie über Ihre vielleicht noch diffusen Ideen und Änderungsimpulse sprechen: Wie verändert sich Ihre Stimmung? Wie fühlt sich all das körperlich an? Welches umfassende Körpergefühl können Sie dazu wahrnehmen? Welche „Eigenart" hat dieses Gefühl - suchen Sie ein passendes Eigenschaftswort (...). Vielleicht entsteht vor Ihrem inneren Auge auch ein Bild, das zu diesem umfassenden Körpergefühl passt?

6. Wenn Sie betrachten, wo Sie jetzt stehen: Was von dem schwer sagbaren Neuen ist etwas deutlicher oder klarer geworden? Welche Worte helfen, dies auszudrücken? (...)

7. Spüren Sie einmal nach: Was ist der Kern, das Lebendigste an Ihrer neuen Idee? Drücken Sie dies in einem Satz aus (,Kernsatz“).

8. Lassen Sie auch Ihr Körpergefühl sprechen: Können Sie Ihrem Satz voll und ganz zustimmen? Was daran stimmt, was stimmt noch nicht? Fehlt noch etwas?“ (Deloch, 2017, S. 130) 
Das Augenmerk von TAE und ECC liegt auf dem sprachlichen Explizieren von neuen Sichtweisen und Konzepten und darin, neue Bedeutungen in Worten zu entdecken, die zunächst unlogisch klingen mögen, am Ende aber neue Facetten beinhalten. Die zentrale Aufgabe der Coach besteht im absoluten Zuhören (Listening). So

„sagt der Coach gelegentlich zurück, was er verstanden hat und verwendet dabei auch die Schlüsselworte der Akteurin. Ein Sicherstellen und Wiedergeben dieser Worte ermöglicht es, möglichst nah am noch unklaren Rand des Denkens zu verweilen (...) Der Coach unterstützt diesen Prozess noch weiter, indem er die Überlegungen der Akteurin aufschreibt.“ (Deloch, 2017, S. 124) 


\subsection{Zwischenfazit}

Passmore (2007) beschreibt in seinem integrativen Coaching-Modell drei „Ströme“, denen Coach und Coachee sich in ihrer gemeinsamen Arbeit widmen sollten (vgl. Ryba, 2018, S. $52)$ :

1. Der behaviorale Strom: Im Vordergrund steht das Verhalten der Coachee, Verhaltensänderungen sollen durch Förderung der Problemlösungs- und Planungskompetenzen der Klientin unterstützt werden.

2. Die bewusste Kognition: Hier geht es um kognitive Muster der Klientin, die ihr Handeln bestimmen. Diese gilt es zu erkennen, zu reflektieren und gegebenenfalls durch entsprechende kognitiv-verhaltenstherapeutisch orientierte Techniken zu verändern.

3. Die unbewusste Kognition: Gemeint sind damit tief verwurzelte, nicht bewusste emotionale und motivationale Aspekte, die das Verhalten des Individuums beeinflussen. Auch diesen „Strom“ gilt es im Coaching zu berücksichtigen und durch entsprechende Interventionen und Techniken zu adressieren.

Aus neurowissenschaftlicher Sicht kann der Strom der unbewussten Kognition besonders gut über körperorientiertes Vorgehen bearbeitet werden, so dass Ryba (2018, S. 476) fordert:

„Die Integration des Körpers in das Coaching erscheint zwingend notwendig! Hier braucht es coachingsspezifische Konzepte.“

Focusing bzw. Experiencing scheint, neben anderen Verfahren, die eine Verbindung zwischen Körpererleben oder emotionalem Erleben und kognitiver Verarbeitung herstellen, geeignet, um die von Ryba geforderte Integration des Körpers zu ermöglichen, und zwar auf eine Art und Weise, die Spüren und Denken bzw. Affekt und Kognition zusammenführt, so dass daraus Klarheit oder Neues entstehen kann. Focusing und das Konzept des Felt Sense machen das unmittelbare Problemerleben einer Person direkt fassbar, aus dem sich dann der „,vorwärtstragende Problemlöse-Prozess“ entwickelt (Feuerstein \& Müller, 1998, S. 42).

„Damit wird es möglich, das Programm der Personzentrierung als Zentrierung auf den „Prozess in der Person“ auch methodisch auf den Punkt zu bringen.“(ebd.)

Auch wenn Sachse eine psychologische Theorie zum Focusing beschreibt, bleibt für die Forschung problematisch, dass Gendlins originäres Konzept mehr philosophisch und phänomenologisch als psychologisch begründet ist. Dieses Problem der nur ansatzweise ausgearbeiteten psychologischen Theorie trifft auch für andere hier vorgestellte körperorientierte oder achtsamkeitsbasierte Orientierungen zu, so dass ein Rückgriff auf diese Verfahren für den Theorie-Diskurs nur begrenzt hilfreich ist. Ausgereifte Theoriemodelle finden sich in psychoanalytischen Konzepten, im hypnosystemischen Ansatz und im prozess-experienziellen emotionsfokussierten Ansatz (PE-EFT). Die größte Nähe zu Gendlins experienziellem Ansatz besteht beim PE-EFT-Modell, wobei dort die Spezifika des Felt Sense und des Felt Shift nicht 
als grundlegend zum Tragen kommen. Auch im hypnosystemischen Ansatz wird dem Körpererleben eine Rolle zugeschrieben, die gewisse Ähnlichkeiten mit der Rolle des Körpers im experienziellen Ansatz hat.

So sollen hier nochmals Parallelen und Unterschiede zwischen zentralen für die intrapersonale Veränderung relevanten Annahmen dieser beiden zuletzt genannten Ansätze und den Konzepten von Gendlin und von Sachse gegenübergestellt werden. Der Übersichtlichkeit halber wird auf die Quellenangaben verzichtet, sie finden sich in den jeweiligen Kapiteln dieser Arbeit:

\begin{tabular}{|c|c|c|c|}
\hline $\begin{array}{l}\text { Experienzieller } \\
\text { Ansatz (Gendlin) }\end{array}$ & $\begin{array}{l}\text { Experienzielle Theo- } \\
\text { rie (Sachse) }\end{array}$ & $\begin{array}{l}\text { Prozess-Experien- } \\
\text { zielle Emotionsfo- } \\
\text { kussierte Theorie } \\
\text { (Greenberg) }\end{array}$ & $\begin{array}{l}\text { Hypnosystemische } \\
\text { Theorie (Schmidt) }\end{array}$ \\
\hline \multicolumn{4}{|c|}{ Epistemologische Grundlagen } \\
\hline $\begin{array}{l}\text { Humanistische } \\
\text { Psychologie, Exis- } \\
\text { tenzphilosophie, } \\
\text { Phänomenologie }\end{array}$ & $\begin{array}{l}\text { Humanistische Psy- } \\
\text { chologie, Kognitions- } \\
\text { psychologie }\end{array}$ & $\begin{array}{l}\text { Humanistische Psy- } \\
\text { chologie, mit Ele- } \\
\text { menten der Emoti- } \\
\text { onspsychologie und } \\
\text { des dialektischen } \\
\text { Konstruktivismus }\end{array}$ & $\begin{array}{l}\text { Systemische Theorie } \\
\text { und Therapie, Hyp- } \\
\text { nose }\end{array}$ \\
\hline \multicolumn{4}{|c|}{ Persönlichkeitskonzept } \\
\hline $\begin{array}{l}\text { Aktualisierungs- } \\
\text { tendenz; Selbst- } \\
\text { konzept und orga- } \\
\text { nismisches Wert- } \\
\text { system }\end{array}$ & $\begin{array}{l}\text { Aktualisierungsten- } \\
\text { denz, Selbstkonzept } \\
\text { und organismisches } \\
\text { Wertsystem }\end{array}$ & $\begin{array}{l}\text { Selbstbestimmung } \\
\text { und Orientierung } \\
\text { zur Weiterentwick- } \\
\text { lung als Grundlage. } \\
\text { Dialektisches Zu- } \\
\text { sammenspiel inne- } \\
\text { rer Teile, unter an- } \\
\text { derem affektiv-mo- } \\
\text { tivationaler und } \\
\text { kognitiver Teile }\end{array}$ & $\begin{array}{l}\text { Mensch als sich selbst } \\
\text { organisierendes Sys- } \\
\text { tem. Zentrale Prinzi- } \\
\text { pien: Autopoiese, } \\
\text { Selbstreferenz. } \\
\text { Differenzierung zwi- } \\
\text { schen bewusster Erle- } \\
\text { bensinstanz und un- } \\
\text { willkürlichen körperli- } \\
\text { chen und seelischen } \\
\text { Prozessen }\end{array}$ \\
\hline \multicolumn{4}{|l|}{ Affekte, Emotionen } \\
\hline $\begin{array}{l}\text { Gefühle als kom- } \\
\text { plexe, implizite } \\
\text { Wissensform }\end{array}$ & $\begin{array}{l}\text { Affekte als Ergebnis } \\
\text { präkognitiver, } \\
\text { perzeptuell-sensumo- }\end{array}$ & $\begin{array}{l}\text { Affektiv-motivatio- } \\
\text { nale bzw. emotio- } \\
\text { nale Schemata sind } \\
\text { wesentlich beteiligt }\end{array}$ & $\begin{array}{l}\text { Emotionen und Af- } \\
\text { fekte sind nicht Be- } \\
\text { standteil des Konzepts, }\end{array}$ \\
\hline
\end{tabular}




\begin{tabular}{|c|c|c|c|}
\hline & $\begin{array}{l}\text { torischer Appraisal- } \\
\text { prozesse. Emotionen } \\
\text { als sekundäre Reprä- } \\
\text { sentation von Affek- } \\
\text { ten }\end{array}$ & $\begin{array}{l}\text { an selbstorganisie- } \\
\text { renden Prozessen, } \\
\text { affektiv-motivatio- } \\
\text { nale und kognitive } \\
\text { Aspekte des Erle- } \\
\text { bens lassen sich } \\
\text { kaum voneinander } \\
\text { trennen }\end{array}$ & $\begin{array}{l}\text { wohl aber die Diffe- } \\
\text { renzierung zwischen } \\
\text { explizitem, narrativem } \\
\text { Gedächtnis und impli- } \\
\text { zitem Gedächtnis, des- } \\
\text { sen Prozesse durch } \\
\text { Gefühle und Imagina- } \\
\text { tionsprozesse gekenn- } \\
\text { zeichnet sind }\end{array}$ \\
\hline \multicolumn{4}{|l|}{ Körper } \\
\hline $\begin{array}{l}\text { Körper als Träger } \\
\text { impliziten Wis- } \\
\text { sens. Felt Sense als } \\
\text { eigene Kategorie, } \\
\text { die Affekte, Kör- } \\
\text { perspüren und } \\
\text { Kognitionen ver- } \\
\text { eint }\end{array}$ & $\begin{array}{l}\text { Körperliche Reaktio- } \\
\text { nen als Ergebnis af- } \\
\text { fektiver Verarbei- } \\
\text { tungsprozesse und In- } \\
\text { formationsträger af- } \\
\text { fektiver Bedeutungen } \\
\text { (felt senses) }\end{array}$ & $\begin{array}{l}\text { Emotionale Sche- } \\
\text { mata können auch } \\
\text { körperlich reprä- } \\
\text { sentiert werden. Sie } \\
\text { können aber ebenso } \\
\text { verbal, visuell, } \\
\text { perzeptuell oder } \\
\text { motivational reprä- } \\
\text { sentiert werden }\end{array}$ & $\begin{array}{l}\text { Unwillkürliche Kör- } \\
\text { persignale als Aus- } \\
\text { druck des impliziten } \\
\text { Gedächtnisses tragen } \\
\text { bei zur Realitätskon- } \\
\text { struktion und zur } \\
\text { Selbststeuerung }\end{array}$ \\
\hline \multicolumn{4}{|l|}{ Störungskonzeption } \\
\hline $\begin{array}{l}\text { Die freie Interak- } \\
\text { tion zwischen im- } \\
\text { plizit gefühlter Be- } \\
\text { deutung und äuße- } \\
\text { rer Symbolisierung } \\
\text { ist gestört durch } \\
\text { alte, verfestigte } \\
\text { Strukturen (struk- } \\
\text { turgebundenes } \\
\text { Selbsterleben) }\end{array}$ & $\begin{array}{l}\text { Negative affektive } \\
\text { Erfahrungen bilden } \\
\text { ein negatives bzw. } \\
\text { dysfunktionales af- } \\
\text { fektives Schema, das } \\
\text { zu ungünstigen Ver- } \\
\text { arbeitungsprozessen } \\
\text { führt }\end{array}$ & $\begin{array}{l}\text { Maladaptive emoti- } \\
\text { onale Prozesse ba- } \\
\text { sieren auf einem er- } \\
\text { fahrungsbasierten } \\
\text { falschen Verständ- } \\
\text { nis von Situationen } \\
\text { und wirken so dys- } \\
\text { funktional }\end{array}$ & $\begin{array}{l}\text { Inkongruenzen werden } \\
\text { als Störung erlebt und } \\
\text { entstehen bei einer } \\
\text { Diskrepanz zwischen } \\
\text { bewusstem, willkürli- } \\
\text { chem Erleben und un- } \\
\text { willkürlichem körper- } \\
\text { lichem oder seeli- } \\
\text { schem Erleben }\end{array}$ \\
\hline \multicolumn{4}{|c|}{ Veränderungskonzeption } \\
\hline $\begin{array}{l}\text { Experiencing als } \\
\text { vorwärtstragender } \\
\text { Prozess im Sinn ei- } \\
\text { ner freien, nicht } \\
\text { strukturgebunde- } \\
\text { nen Interaktion } \\
\text { zwischen implizit }\end{array}$ & $\begin{array}{l}\text { Verarbeitung erfolgt } \\
\text { auf der Grundlage } \\
\text { motivational-affekti- } \\
\text { ver und kognitiver } \\
\text { Schemata, die in einer } \\
\text { teilweisen Unabhän- } \\
\text { gigkeit zueinander }\end{array}$ & $\begin{array}{l}\text { Bewusstes Wahr- } \\
\text { nehmen emotiona- } \\
\text { ler Reaktionen. } \\
\text { Verändern von dys- } \\
\text { funktionalen Sche- } \\
\text { mata durch alterna- }\end{array}$ & $\begin{array}{l}\text { Aufmerksamkeitsfo- } \\
\text { kussierung zur Nutz- } \\
\text { barmachung von un- } \\
\text { willkürlichen Prozes- } \\
\text { sen, um eine möglichst } \\
\text { optimale Koordination } \\
\text { mit willkürlichen Ich- }\end{array}$ \\
\hline
\end{tabular}




\begin{tabular}{|c|c|c|c|}
\hline $\begin{array}{l}\text { gefühlten Bedeu- } \\
\text { tungen und äuße- } \\
\text { ren Symbolen. } \\
\text { Neues entsteht } \\
\text { durch Symbolisie- } \\
\text { rung - und damit } \\
\text { dem Explizieren - } \\
\text { von bis dato impli- } \\
\text { ziten Bedeutungs- } \\
\text { inhalten des Felt } \\
\text { Sense, so dass sich } \\
\text { ein Felt Shift ein- } \\
\text { stellt. }\end{array}$ & $\begin{array}{l}\text { stehen. Veränderung } \\
\text { erfolgt durch das Fin- } \\
\text { den von kognitiven, } \\
\text { in Sprache gefassten } \\
\text { Entsprechungen der } \\
\text { affektiven Bedeutun- } \\
\text { gen. Kognitive Re- } \\
\text { präsentation affekti- } \\
\text { ver Schemata führt } \\
\text { zur Aktivierung von } \\
\text { Emotionen, die Per- } \\
\text { son spürt die Bedeu- } \\
\text { tung der kognitiven } \\
\text { Repräsentation. }\end{array}$ & $\begin{array}{l}\text { tive, adaptive emo- } \\
\text { tionale Reaktionen. } \\
\text { Emotions-Wahr- } \\
\text { nehmung geschieht } \\
\text { durch experienziel- } \\
\text { les Erforschen inne- } \\
\text { ren Erlebens. }\end{array}$ & $\begin{array}{l}\text { Prozessen herzustel- } \\
\text { len. }\end{array}$ \\
\hline
\end{tabular}

Tabelle 3: Vergleich zentraler theoretischer Konstrukte

Zusammenfassend kann festgestellt werden, dass Sachse und Greenberg jeweils unterschiedliche Wege in der Weiterentwicklung des experienziellen Ansatzes gehen, beide aber Theorien entwickeln, die als kompatibel mit aktuellen emotionspsychologischen und kognitionspsychologischen Erkenntnissen betrachtet werden können. Der zentrale Unterschied besteht in der Rolle, die dem Körpererleben jeweils zukommt. Die Nähe der Theoriemodelle zeigt sich auch darin, dass sowohl im experiencingorientierten bzw. focusingorientierten Ansatz als auch im emotionsfokussierten Ansatz die Experiencing Skala als Instrument zum Prozessrating empfohlen wird.

Dem hypnosystemischen Ansatz liegen andere erkenntnistheoretische Grundannahmen zugrunde als den experienziellen bzw. emotionsfokussierten Verfahren, auch spielen Affekte bei der Konzeptionierung keine zentrale Rolle. Trotzdem beschreibt er aus systemisch-konstruktivistischer Sicht internale Interaktionsprozesse, die zwischen zwei unterschiedlichen und unterschiedlich bewussten - Verarbeitungs- und Bewertungssystemen stattfinden und die dem körperlichen Erleben eine ähnliche Rolle zuweisen wie das Experiencing-Konzept, ohne dabei aber den Felt Sense oder ein vergleichbares spezifisches Konstrukt zu integrieren.

\subsection{Experiencing-Scale}

Um experienzielle Prozesse beurteilen zu können, wurden verschiedene Messinstrumente entwickelt. Sie unterscheiden sich nicht nur inhaltlich, sondern auch hinsichtlich des methodischen Vorgehens. So ist beispielsweise die von Iberg (1996) entwickelte Skala zur Erfassung von Focusingprozessen als Post-Session Fragebogen für Klientinnen konzipiert. Neben den Fragen zur Operationalisierung besteht die Schwierigkeit hier, wie bei allen Post-Session- 
Fragebögen, darin, dass ein solches Vorgehen bei Coachs und Coachees wenig akzeptiert ist und daher eine Erhebung nur schwer durchführbar ist. Praktikabel hingegen erscheint der Einsatz eines Instruments, das im Rahmen der Auswertung von Audiographien bzw. Transkripten von Coachinggesprächen genutzt werden kann.

Als das bekannteste Instrument zur Erfassung des Experiencing bzw. unmittelbarer emotionsbezogener Prozessvariablen auf der Mikroebene gilt die bereits im Kontext der ExperiencingForschung (Kapitel 3.4) erwähnte Experiencing-Skala EXP (Klein et al, 1969; vgl. Benecke, 2014, S. 631). Sie wird von Leuzinger-Bohleber, Benecke \& Hau (2015, S.195) auch für die Untersuchung von Coachingprozessen bzw. Coachinggesprächen empfohlen. Die Skala wurde von Dahlhoff \& Bommert (1978) ins Deutsche übertragen und beschreibt das

„Ausmaß, in dem der Patient in seinen Äußerungen eine Bezugnahme auf sein aktuelles Erleben zeigt. Eine solche Bezugnahme ist gegeben, wenn der Patient sich auf seine eigenen Gefühle und Reaktionen - so wie er sie im Moment erfährt - bezieht, und / oder wenn er auf der Suche nach der persönlichen Bedeutung ist, die Ereignisse, Situationen, Gefühle und Ideen für ihn haben.“(ebd., S. 73)

Das Experiencing der Klientin kann dabei sowohl als Wirkfaktor als auch als Prädiktor für Behandlungsergebnisse betrachtet werden (Pascual-Leone \& Yeryomenko, 2017). Yeryomenko (2012) kommt in einer Meta-Analyse zur Experiencing-Skala zu dem Ergebnis, dass das mit diesem Instrument erfasste Erleben als kleiner bis mittlerer Prädiktor den Behandlungserfolg von Psychotherapien vorhersagen kann (Korrelationskoeffizient zwischen $r=.11$ und $\mathrm{r}=.36$; ebd., S. 40).

Neben der Experiencing-Skala existieren noch weitere Instrumente zum Erfassen inneren Erlebens: Lex \& Bischkopf (2012, S. 207) erwähnen die von Hohage entwickelte Rating-Skala der emotionalen Einsicht, bei der emotionale Einsicht als eine spezifische Form der Selbstreflexion betrachtet wird, allerdings liegt der Schwerpunkt des Konzepts hier mehr auf der Integration von Ambiguität und nicht so sehr auf der Beschreibung des inneren Erlebens. Auch Greenberg hat mit der Client Expressed Emotional Arousal Scale-III (vgl. Benecke, 2014, S. 633) und mit der Emotion Productivity Scale (vgl. ebd.) Messinstrumente zur Erfassung emotionalen Erlebens entwickelt, die allerdings nicht nur auf Experiencing im engeren Sinn abzielen. Ein in der personzentrierten Beratung und Therapie verbreitetes Instrument ist die von Truax \& Carkhuff (1967) entwickelte Skala der Selbstexploration, anhand derer verschiedene Aspekte der Selbstexploration eingeschätzt werden können. Sachse (1992, S. 135) weist allerdings auf die „Mehrdimensionalität“ des Selbstexplorations-Konzepts hin, so dass die Relevanz der Skala für die Erfassung von Experiencing nur schwer abzuschätzen sei. Auch ist der Begriff der Selbstexploration zu unterscheiden vom Begriff des Selbsterlebens, wie Dahlhoff \& Bommert (1978, S. 65) ihn im Forschungs- und Trainingsmanual zur ExperiencingSkala als Übersetzung für das englische Experiencing verwenden. Selbsterleben ist in diesem Manual definiert als gefühlsmäßiges, vorbegriffliches Erleben, bzw. als das

„konkrete, im Augenblick vor sich gehende Erleben eines Individuums, bei dem die 
Aufmerksamkeit auf einen zugrundeliegenden gefühlten Erlebnisgegenstand gerichtet ist. Dieser Gegenstand muss nicht unbedingt vollständig verbal fassbar sein, es ist für das Individuum mehr eine körperlich fühlbare Beziehung: eine gefühlte, unmittelbar gegenwärtige persönliche Bedeutung von Dingen und Erlebnissen.“(ebd.)

Die Experiencing-Skala besteht aus 7 Stufen, in denen die Abstufungen des ExperiencingProzesses operationalisiert sind, wobei die Stufen 1 bis 3 einen innerlichen Abstand zum Selbsterleben beschreiben und der eigentliche Experiencing-Prozess im Sinn einer Wahrnehmung des unmittelbaren Fühlens und Erlebens von einem innen liegenden Standpunkt heraus auf den Stufen 4 bis 7 dargestellt wird (Lex \& Bischkopf, 2012, S. 208). Für die Beschreibung der einzelnen Stufen werden in erster Linie direkt im Kommunikationsverhalten beobachtbare Variablen verwendet.

Die Dimensionen der Stufen sind:

„Stufe1: Der Klient schildert Ereignisse in allgemeiner, unpersönlicher Weise. Seine Äußerungen sind von unbeteiligter Art, so als ob sie von einem Fremden stammen. Eine persönliche Anteilnahme an seinen Mitteilungen ist nicht ersichtlich.

Stufe 2: Der Klient lässt in seinen Äußerungen eine persönliche Rolle erkennen, jedoch nimmt er auf sein Fühlen nur indirekt Bezug, indem er es zum Beispiel zur näheren Verdeutlichung eines Sachverhalts heranzieht. Gefühle und persönliche Reaktionen werden eher als entfernte, externe Ereignisse behandelt und charakterisiert, und es wird nur indirekt oder abstrakt darauf Bezug genommen. Ein intellektuelles Interesse bzw. ein allgemeines, oberflächliches Beteiligtsein ist vorhanden, jedoch ist die Bezugnahme des Klienten auf sein Fühlen allgemein-abstrakt oder distanziertversachlicht und nicht auf ein tieferes Eindringen in die persönliche Bedeutung ausgerichtet.

Stufe 3: Der Klient bezieht sich zwar auf sein Fühlen und Erleben, jedoch werden diese überwiegend als Verhaltensweisen oder Situationen dargestellt oder als in enger Verknüpfung mit diesen Verhaltensweisen und Situationen stehend geschildert. Die Mitteilungen über das Fühlen und Erleben erfolgen gleichsam von einem äußeren Bezugspunkt des Klienten. Eine Bezugnahme auf das Fühlen und Erleben erfolgt in sehr begrenztem Umfang, wird nur knapp zum Ausdruck gebracht und nicht tiefgehend bearbeitet.

Stufe 4: Der Klient bezieht sich in seinen Äußerungen auf seinen inneren Bezugsrahmen. Er versucht, diesen zur Grundlage seiner Mitteilungen zu machen. Er betrachtet Ereignisse, Situationen und sein Fühlen nicht mehr von außen, sondern er entwickelt eine innerlich gefühlte Bedeutung, auf die er in seinen Mitteilungen Bezug nimmt. Bei der Beschreibung seiner Gefühle der Vergangenheit und Gegenwart schöpft der Klient aus diesem momentanen Erleben und Beteiligtsein. Er bemüht sich, sein Fühlen nicht nur zu schildern, sondern auch zu erarbeiten, was diese Ge- 
fühle für ihn bedeuten. Oft besteht auch der Eindruck, dass mehr im Klienten vorgeht, als er im Moment erfassen kann, es sind noch nicht ausformulierte Erlebnisinhalte vorhanden.

Stufe 5: Der Klient konzentriert sich auf seinen inneren Bezugsrahmen; dabei werden innere Erlebnisse weiterberarbeitet, erforscht und verdeutlicht. Jetzt kann sich der Klient auf bisher vage, nicht ausformulierbare aber bedeutsame Aspekte seines unmittelbaren Erlebens beziehen und kann diese allmählich differenzieren. Er gewinnt einen detaillierten Bezug zu seinen aktuellen Erlebnisvorgängen.

Stufe 6: Der Klient erfährt durch die Arbeit an seinem unmittelbaren Erleben Gefühlsänderungen oder eine Verlagerung gefühlter Bedeutungen. Einzelne aktuelle Erlebnisinhalte ändern sich in ihrer Bedeutung für den Klienten oder werden ihm eigentlich erst klar; so kann er vorher unklare bzw. bruchstückhafte Erlebnisinhalte durch sein unmittelbares Erleben für sich deutlich machen. Der Klient ist in der Lage, diese Bedeutungsänderungen zu überdenken und zu verarbeiten. Er macht sie zu Grundlage für seine erlebnismäßig erarbeiteten Absichten und Vorstellungen.

Stufe 7: Der Klient verfügt über ein klares Bild von seinem unmittelbar ablaufenden Erleben und kann dieses in seinen Äußerungen vermitteln. Er ist in der Lage, sich in seinem wechselnden Erleben zu bewegen, die Bedeutung des Erlebens für ihn selbst $\mathrm{zu}$ verstehen und Bedeutungsänderungen in seinen Bezugsrahmen zu integrieren. Sein unmittelbares aktuelles Erleben, der Wechsel von Bedeutungsschwerpunkten und auftretende Bedeutungsänderungen sind ihm eine vertrauenswürdige Basis für Änderungen in seinem Erleben und Verhalten.“ (Dahlhoff \& Bommert, 1978, S. 73)

Im Manual zur Skala unterscheiden die Autoren zwischen dem Modus und dem Spitzenwert (ebd., S. 78). Während der Modus den allgemeinen, häufigsten Stufenwert der zu beurteilenden Episode kennzeichnet, bezieht sich der Spitzenwert auf die höchste in der zu beurteilenden Einheit vorkommende Stufe. In explorativen Untersuchungen erwies sich der Spitzenwert hinsichtlich der Reliabilität als zuverlässiger im Vergleich zum Modus, so dass die Autoren empfehlen, gegebenenfalls auf den Spitzenwert zurückzugreifen (Laux \& Bischkopf, 2012, S. 208). Für den Spitzenwert geben Dahlhoff \& Bommert (1978, S. 79) eine Beurteilerinnenübereinstimmung von $r=0,83$, und für den Modus eine Beurteilerinnenübereinstimmung von $r=0,72$. Der Re-Rate-Koeffizient liegt bei $r=0,62$ für den Spitzenwert und bei $r=0,55$ für den Modus und damit in dem auch bei anderen Prozessskalen üblichen Rahmen.

Mathieu-Coughlan \& Klein (1984, S. 221) beschreiben zu den einzelnen Stufen des Experiencing auf Seiten der Klientin auch ein paralleles Experiencing der Therapeutin, das folgendermaßen dargestellt werden kann (Übertragung ins Deutsche durch den Autor): 


\begin{tabular}{|c|c|c|}
\hline Stufe & Klientin bezieht sich auf... & Therapeutin (Coach) verhält sich... \\
\hline 1 & $\begin{array}{l}\text { äußere Ereignisse, ohne direkte Beteili- } \\
\text { gung der Klientin }\end{array}$ & unpersönlich, losgelöst von der Person \\
\hline 2 & $\begin{array}{l}\text { äußere Ereignisse, mit Beteiligung der } \\
\text { Klientin, verhaltensmäßige oder intel- } \\
\text { lektuelle Beschreibung der Gedanken o- } \\
\text { der des Verhaltens der Klientin }\end{array}$ & $\begin{array}{l}\text { interessiert, mit gedanklichen Bezügen } \\
\text { zur Person der Klientin }\end{array}$ \\
\hline 3 & $\begin{array}{l}\text { ihre Reaktionen auf äußere Ereignisse. } \\
\text { Begrenzt oder auf einer Verhaltensebene } \\
\text { berichtet sie auch über ihre Gefühle }\end{array}$ & $\begin{array}{l}\text { reaktiv. Die Coach beschreibt die wahr- } \\
\text { genommenen Gefühle bei der Klientin o- } \\
\text { der bezieht sich auf diese }\end{array}$ \\
\hline 4 & $\begin{array}{l}\text { die Beschreibung der eigenen Gefühle } \\
\text { und persönlichen Erfahrungen }\end{array}$ & $\begin{array}{l}\text { empathisch involviert, die Coach elabo- } \\
\text { riert oder intensiviert die Gefühle und / } \\
\text { oder Assoziationen, die in diesem } \mathrm{Zu}- \\
\text { sammenhang auftauchen }\end{array}$ \\
\hline 5 & $\begin{array}{l}\text { ihre Probleme oder ihre Vorstellungen } \\
\text { bezüglich ihrer Gefühle und persönli- } \\
\text { chen Erfahrungen }\end{array}$ & $\begin{array}{l}\text { bezieht sich auf eigene Gefühle, um die } \\
\text { Gefühle der Klientin zu explorieren }\end{array}$ \\
\hline 6 & $\begin{array}{l}\text { ihre aktuell auftauchenden Gefühle und } \\
\text { deren Auswirkungen im Erleben }\end{array}$ & $\begin{array}{l}\text { nutzt ihre eigenen aktuell auftauchenden } \\
\text { Gefühle, um die Gefühle der Klientin zu } \\
\text { bestätigen bzw. noch klarer herauszuar- } \\
\text { beiten }\end{array}$ \\
\hline 7 & $\begin{array}{l}\text { ihren inneren Bezugspunkt: Sie ist in der } \\
\text { Lage, sich authentisch von einem inne- } \\
\text { ren Bezugspunkt zum nächsten zu bewe- } \\
\text { gen. }\end{array}$ & $\begin{array}{l}\text { „expansiv“, also in die Breite gehend. } \\
\text { Sie integriert alle Elemente der Interak- } \\
\text { tion, einschließlich der Gefühle der Kli- } \\
\text { entin, der eigenen Gefühle und deren ex- } \\
\text { plizitem Inhalt. }\end{array}$ \\
\hline
\end{tabular}

Tabelle 4: Experiencing und Coachverhalten

Allerdings betonen Dahlhoff \& Bommert in ihrem Manual zur Experiencing-Skala (1978, S. 90), dass diese sich ausschließlich auf das Klientinnenverhalten bezieht, und dass daher beim Rating auch einzig die Klientinnenäußerungen zu berücksichtigen sind. Insofern kann das parallele Therapeutinnen- oder Coach-Verhalten bei einer späteren Auswertung von Interesse sein, beim Rating des Experiencing selbst sollte es zunächst keine Beachtung finden.

Vor dem Hintergrund, dass Experiencing als Kontinuum zu betrachten ist, sind die Übergänge zwischen den einzelnen Stufen nicht immer trennscharf zu definieren. Mathieu-Coughlan \& Klein (1984, S. 222) weisen auf folgende Kennzeichen für die einzelnen Übergänge hin: 
- Stufe 3 zu Stufe 4: Die Fähigkeit der Klientin, sich auf ihr Selbsterleben zu beziehen

- Stufe 4 zu Stufe 5: Die Fähigkeit, sich ausgehend vom inneren Erleben (,hold on the felt referent") auf die Probleme und Bereiche der Unklarheit, der Anstrengung und Unsicherheit zu fokussieren

- Stufe 5 zu Stufe 6: Die Erfahrung des Felt Shift oder „,carrying forward“, die eintritt, wenn es gelingt, sich eine Zeit lang mit dem inneren Erleben zu beschäftigen

- Stufe 6 zu Stufe 7: Die Erfahrung von neuen, autonomen und positiveren Gefühlen, die eintritt, nachdem der Felt Shift sich eingestellt hat und eine Lösung erkennbar wurde.

Dahlhoff \& Bommert (1978, S. 75) nennen und begründen für die jeweilige Stufe Beispiele, die das Rating erläutern, diese sind in den Anlagen dieser Arbeit (sh. Anlage 1: ExperiencingSkala: Ratinginstruktionen und Stufenbeispiele) gemeinsam mit der Rating-Instruktion aufgenommen. 


\section{Forschungsprojekt: Methodische Grundlagen und Ziele}

Wie bereits in der Überschrift formuliert, ist diese Arbeit als Studie zum Felt Sense im Coaching konzipiert. Allerdings führen die Themenstellung, die konzeptionellen Ausführungen zum Experiencing und der Diskurs zu der Erkenntnis, dass es zu kurz greifen würde, dazu einzig typische Focusingprozesse, sofern sie im Rahmen von Coaching durchgeführt wurden, zu untersuchen. Drei Aspekte sind dabei maßgeblich:

1. Gendlins Konzeption folgend, ist davon auszugehen, dass ein Felt Sense und ein damit verbundener vorwärtstragender Prozess sich auch im allgemeinen Methodengebrauch einstellen kann. Dies ist insbesondere im Blick auf Coachingprozesse von Interesse, da Coaching sich zum einen generell zu einem schulen- und methodenübergreifenden Format entwickelt hat (vgl. Migge, 2007, S. 29; Greif et al, 2018, S. 2), und da zum anderen zu vermuten ist, dass in der Praxis arbeitsweltlicher Beratung der Einsatz einer eher therapeutisch oder selbsterfahrungsorientiert anmutenden Methode wie Focusing nicht immer anschlussfähig ist

2. Das von Sachse et al (1992) entwickelte Theoriemodell des Experiencing stellt den Felt Sense nicht als eigenständige Kategorie dar, sondern konzipiert ihn als ein Element bzw. Ergebnis der Interaktion zwischen affektiver Verarbeitung und kognitiven Prozessen. Auch wenn Experiencing und Felt Sense damit nicht voneinander losgelöst sind, bedeutet das, dass nicht der Felt Sense, sondern das Experiencing als zentraler Prozess der Veränderung zu sehen ist. Insofern ist es folgerichtig, bei der Untersuchung der Coachingprozesse nicht den Felt Sense im engeren Sinn, sondern das Experiencing als dem Felt Sense übergeordneten Prozess zu betrachten.

3. Das Interesse der Studie gilt neben dem Experiencing auch den intrapersonalen Veränderungsprozessen insgesamt, so dass diesem Aspekt auch im der empirischen Untersuchung Rechnung zu tragen ist.

Wie und mit welchem Ziel nun die Experiencingprozesse bzw. die Veränderungen im Coaching exploriert werden sollen, soll diesem Kapitel näher vorgestellt werden.

\subsection{Einordnung und Ziele der Studie}

Für die Psychotherapieforschung beschreibt Antal (2012, S. 27) drei Phasen, in denen diese sich entwickelt hat: Nach der ersten, klassischen Phase, in deren Zentrum Fallvignetten und Einzelfallbeobachtungen standen, fokussierte sich die Forschung auf Outcome-Fragen, um die Wirksamkeit eines therapeutischen Verfahrens nachzuweisen. Diese zweite Phase war geprägt von Korrelationsstudien, mit deren Hilfe der Erfolgsbeitrag einzelner isolierter Verhaltensweisen oder Persönlichkeitsmerkmale untersucht wurde. Es folgte in einer dritten 
Phase die Beschäftigung mit dem therapeutischen Prozess und dem therapeutischen Einzelfall in Form einer aufwändigen Prozess-Ergebnis-Forschung bzw. einer prozessorientierten Einzelfallforschung.

Für die Coachingforschung weisen Gessnitzer et al (2018) auf einen ähnlichen Verlauf hin:

„Während in den 1990er Jahren und zu Beginn der 2000er noch vor allem Fallstudien und erste Untersuchungen ohne Kontrollgruppen durchgeführt wurden (...), entstanden nur wenige Jahre später bereits erste Reviews (...). Ab 2010 belegen größere Stichproben und erste Metaanalysen endgültig den positiven Effekt von Coaching.“ (ebd., S. 37)

In Analogie zur Psychotherapieforschung kann davon ausgegangen werden, dass die vorliegenden auf die Wirksamkeit von Coaching bezogenen Metaanalysen auch einen Übergang kennzeichnen - weg von der reinen Outcome-Forschung hin zur prozessorientierten Forschung, die sowohl nach Ergebnissen des Coaching fragt als auch nach Prozesselementen und Phänomenen, die im Coachingprozess erkennbar werden. Loebbert, Wegener, Fritze \& Hänseler (2018, S. 13) formulieren dazu den Anspruch, „Schlussfolgerungen für eine optimale Gestaltung von praktischen Coaching-Interventionen zu ermöglichen“ und ,in gewissem Unterschied zu früheren schulmäßigen, zumeist aus therapeutischen Ansätzen entwickelten Konzepten einen Beitrag zu einer systematischen Coaching-Wissenschaft" einzubringen.

In dieser Hinsicht will diese Studie einen Beitrag leisten: Unter der Prämisse der allgemeinen Wirksamkeit von Coaching sollen im Coaching stattfindende Veränderungsschritte in den Blick genommen werden. Dabei soll die Frage nach dem Felt Sense dazu dienen, bisher möglicherweise noch nicht spezifisch beschriebene Aspekte des Experiencing bzw. der Verbindung zwischen Denken, Verhalten und innerem Erleben zu entdecken beziehungsweise genauer zu verstehen und daraus Implikationen für die Coachingpraxis abzuleiten. Analog zu Cooper et al (2010, S. 246), die darauf hinweisen, dass Therapieforschung eine wesentliche Rolle spielt, um die therapeutische Praxis zu verbessern, soll diese Arbeit dazu dienen, durch Theorie und Wissenschaft auch die Praxis des Coaching weiterzuentwickeln.

Die Fragestellung dieser Arbeit zielt primär auf experienzielle Phänomene und Veränderungsprozesse im Coaching ab. Es sollen, falls erkennbar, Zusammenhänge und Muster herausgearbeitet werden, und gegebenenfalls auch Indikationen, wann ein experiencing-orientiertes Vorgehen im Coaching besonders hilfreich erscheint.

Bereits im einführenden Kapitel zur Themenstellung dieser Arbeit wurden Leitfragen formuliert, die hier nochmals aufgegriffen und priorisiert werden sollen: Primär geht es um die Frage, welche Rolle der Felt Sense bzw. das Experiencing im Coaching spielt. Dazu soll untersucht werden, was Coaching-Klienten im Rahmen des Coaching über ihre aktuellen inneren Prozesse berichten, um daraus Erkenntnisse über das intrapersonale Veränderungsgeschehen im Kontext von Experiencing abzuleiten.

Diese Fragestellungen sind in gewisser Weise unabhängig von der jeweiligen Methode oder Schule zu sehen, an der der Coach sich bei seiner Arbeit orientiert, und das Interesse gilt dabei 
zunächst weniger den Kontextbedingungen oder dem Coachverhalten als vielmehr den intraindividuellen Prozessen auf Seiten des Klienten. Damit soll eine Forderung von Elliot et al (2019) aufgegriffen werden, die mit Blick auf Experiencing die Notwendigkeit betonen, besonders die „three-way interaction between brain processes, behavior and experience“ zu untersuchen. Auch Gendlin formuliert eine ähnliche Erwartung an die Forschung, indem er dazu auffordert, sich nicht in erster Linie mit Methoden zu beschäftigen, sondern vielmehr Prozesse zu untersuchen, und zwar überall dort, wo es um intraindividuelle Veränderung geht (Gendlin, 1986).

Entsprechend verfolgt diese Studie nicht das Ziel, den Verlauf eines Focusingprozesses im Coaching darzustellen, und es geht auch nicht darum, die spezifische Wirksamkeit von Focusing im Coaching nachzuweisen. Diese Aspekte wären für die weitere Fundierung von Focusing im Coaching sicherlich von Interesse, sie würden aber den gestellten Fragen und dem Anliegen, auch über die spezielle Methode des Focusing hinaus einen Beitrag zur CoachingWissenschaft zu leisten, nicht gerecht werden. Konkret geht es darum, anhand exemplarischer realer Coachingprozesse diejenigen Subprozesse zu explorieren, die Hinweise auf Experiencing und einen damit zusammenhängenden Veränderungsprozess geben. Daraus soll abgeleitet werden, welche Facetten des Experiencing über den untersuchten Subprozess hinaus auch für andere Prozesse wünschenswert wären und welche Themen und Aspekte im Coaching durch einen Experiencing-Prozess aufgegriffen werden können. So sollen Erkenntnisse über ein möglicherweise erstrebenswertes experiencingorientiertes Vorgehen im Coaching gewonnen werden.

Nach Koch (2006, S. 4)

„eignen sich qualitative Untersuchungen aufgrund der offenen, weniger strukturierten Methoden dazu, Neues und Unbekanntes zu erforschen. Dabei handelt es sich häufig auch um das Erkennen von Unbekanntem in scheinbar bekannten Erfahrungswelten.“

Wenn diese Studie sich mit Veränderung im Coaching im Allgemeinen und mit Experiencing im Speziellen beschäftigt, taucht sie ein in eine zunächst bekannt erscheinende Erfahrungswelt. Gleichzeitig stellt sie die Frage, ob nicht auch in dieser Erfahrungswelt neue Aspekte zu entdecken sind, die die Kenntnis über die Veränderungsvorgänge im Coaching ergänzen oder vertiefen können. Der offene und explorierende Ansatz qualitativer Forschung scheint dazu gut geeignet zu sein, denn eine qualitative Herangehensweise ermöglicht ein detailliertes Verstehen eines Problems:

"This qualitative understanding arises out of studying a few individuals and exploring their perspectives in great depth whereas the quantitative understanding arises from examining a large number of people and assessing responses to a few variables." (Creswell \& Plano Clark, 2011, S. 11)

Als zentrales Prinzip qualitativer Forschung gilt die Offenheit im Sinn einer unvoreingenom- 
menen Exploration des Forschungsfelds, bei der im Vorfeld keine Selektion durch methodische Filtersysteme oder vorab formulierte Hypothesen betrieben wird (Lamnek, 2005, S. 20). Diese Offenheit bezieht sich nicht nur auf die Untersuchungsperson, sondern auch auf den Forschungsgegenstand und die Forschungsmethoden. Sie fordert vom Forschenden eine Unvoreingenommenheit gegenüber möglichen Ergebnissen und Erkenntnissen. Dem gegenüber steht, dass jeder Forscher von Erkenntnis leitenden Menschenbildannahmen beeinflusst ist, derer er sich bewusst sein sollte (ebd., S. 32), und dass, abhängig vom jeweiligen Erkenntnisinteresse, gerade auch in interpretativen Forschungsprozessen eine Vorab-Strukturierung anhand vorgegebener Theorien und Kategorien (Deppermann, 2008, S. 18) oder ein iterativer Abgleich zugrundeliegender Theorien mit dem empirischen Material (vgl. Greif \& Riemenschneider-Greif, 2018, S. 129) sinnvoll sein kann, wie das auch bei der hier zum Einsatz kommenden Task Analyse der Fall ist.

\subsection{Change Process Research}

Für das Coaching fordern Künzli \& Stulz (2011, S. 163) mehr individuumsorientierte Forschung, die nicht nur durchschnittliche Veränderungen innerhalb einer Stichprobe reflektiert, sondern im Einzelfall eine differenzierte Betrachtung des individuellen Prozesses vornimmt. Prozess wird in diesem Zusammenhang verstanden als

„eine zeitlich geordnete Sequenz von Ereignissen, wobei jedes Element der Sequenz die nachfolgenden Elemente zum Teil determiniert.“ (Reinecker, 1984, S. 159)

Aus den vorhergehenden Kapiteln wurde schon deutlich, dass die Erforschung solcher Prozesse im Coaching noch nicht auf eine lange Geschichte verweisen kann. Vielmehr greift sie zurück auf die umfangreiche Tradition der Psychotherapie-Prozessforschung, deren Hauptanliegen es ist, Auslöser, Effekte oder Elemente des (therapeutischen) Veränderungsprozesses zu identifizieren, zu beschreiben, zu erklären und somit auch vorhersagen zu können (Graf, 2015, S. 6). Dabei untersucht sie Elemente wie das Verhalten des Coachs, das Verhalten des Klienten oder die zwischen den beiden stattfindende Interaktion während des Coaching.

„Prozessforschung wird definiert als die Analyse der Interaktion zwischen den Systemen Therapeut und Klient. Ihr Ziel ist es, den Veränderungsprozess innerhalb dieser Interaktion der beiden Systeme zu identifizieren. Ein solcher Forschungsansatz umfasst das Verhalten und die Erfahrungen der beiden Systeme, die den Veränderungsprozess betreffen, sowohl innerhalb als auch außerhalb der Therapiesitzung.“ (Schindler, 1991, S. 43, zitiert nach Greenberg \& Pinsof, 1986)

Nach Czogalik (1999, S. 162) kann Prozessforschung sowohl Aspekte der Interaktionsforschung als auch Aspekte der Episodenforschung enthalten. Während die Interaktionsforschung sich mit der Erfassung interaktioneller Verhaltensweisen zwischen Coach und Coachee beschäftigt und damit auch mit der Frage, welche Interaktionen des Coachs zu welchen 
Veränderungen beim Coachee führen, versucht die Episodenforschung, diejenigen Ereignisse einzugrenzen und zu beschreiben, die für den Coachingprozess besonders bedeutsam sind. Beide Aspekte finden sich in der Change Process Research (CPR) wieder (vgl. Elliot, 2012a). Um nicht nur Prozessanalysen ohne Ergebnis-Relevanz hervorzubringen, sind auch in der Change Process Research Zusammenhänge zwischen den Prozessvariablen und einer Veränderung im Sinn des Behandlungs- oder Beratungsergebnisses herauszuarbeiten (Caspar \& Jacobi, 2004, S. 398). Dabei können nach Greenberg (1986, S. 6) drei Ebenen unterschieden werden:

1. Unmittelbare Veränderung: Die Veränderung ist in der jeweiligen Coachingsequenz evident

2. Mittelfristige Veränderung: Die Veränderung ist als Ergebnis von einzelnen Coachingsitzungen messbar

3. Finale Veränderung: Die Veränderung ist im Prä/Post/Katamnese-Vergleich erkennbar.

Abhängig von der jeweiligen Ebene der Veränderung, die in einer Untersuchung fokussiert werden soll, können im Rahmen der Change Process Research unterschiedliche methodische Zugänge genutzt werden. Elliot (2010) unterscheidet vier Zugangswege, die auch sich ergänzend zum Einsatz kommen können:

- Quantitativ orientiertes Prozess-Outcome Design: Prüfen von Hypothesen zu Prozess- und Ergebnis-Zusammenhängen

- Helpful-Factors-Design: Klienten beschreiben die aus ihrer Sicht für die Veränderung hilfreichen und relevanten Aspekte der Therapie

- Sequential Process Design: Mikroanalyse sequentieller Interaktionen zwischen Klient und Therapeut

- Significant Event Approach: Analyse bedeutsamer Momente.

Eine fast identische Systematik entwickelt Wegener (2018, S. 23) in seiner prozessorientierten Coaching-Forschungslandkarte:

- Im Prozess-Outcome-Design werden theoretisch postulierte Zusammenhänge zwischen Prozess- und Ergebnisvariablen statistisch überprüft

- Das Helpful-Factors-Design bedient sich der qualitativen Sozialforschung, um neue Aspekte aus der subjektiven Erlebensperspektive von Coach und / oder Coachee zu generieren

- Im Microanalytic-Sequential-Design geht es darum, die Gültigkeit theoretisch abgeleiteter Wirkzusammenhänge im Rahmen detaillierter Auswertungen einzelner Interaktionssequenzen zu überprüfen

- Das Significant-Events-Design verfolgt das Ziel, Theorien zur Entstehung bedeutsamer Ereignisse im Coaching zu entwickeln und bedient sich dazu neben den Daten zu den vorliegenden Coaching-Verläufen ähnlich wie im Helpful-FactorsDesign der subjektiven Einschätzung der Beteiligten. 
Beide Systematiken beruhen auf der aus der psychotherapeutischen Prozessforschung als „Typ-B-Forschung“ bekannten Perspektive, die das Behandlungs- bzw. Beratungsgeschehen als sequentiell und nicht als in sich einheitlichen oder linearen Behandlungsverlauf versteht (Bastine et al, 1989, S. 7; Rice \& Greenberg, 1984, S. 10).

Die Relevanz einzelner Sequenzen bzw. bedeutsamer Ereignisse im Coaching wird besonders von De Haan \& Nieß $(2012,2018)$ betont. Sie bezeichnen solche signifikanten Ereignisse als critical incidents oder critical moments, die von Coachs und ihren Klienten wahrgenommen werden als

"an exciting, tense or significant moment experienced during coaching sessions." (De Haan \& Nieß, 2012, S. 201)

Konkret geht es dabei um:

- Momente des Lernens, bei denen eine neue Einsicht entsteht oder neue Verbindungen oder Perspektiven aufgegriffen werden

- Eine positive oder negative Veränderung in Beziehungen

- Eine Handlung seitens des Klienten oder des Coachs, die in diesem Moment eine hohe Bedeutung hat

- Eine besonders positive oder besonders negative Emotion auf Seiten des Klienten oder auf Seiten des Coachs

- $\quad$ Aufkommende Zweifel, die eine hohe Bedeutung haben (ebd., S. 202).

Die Forschungsergebnisse zeigen, dass Klienten und Coachs in der retrospektiven Betrachtung signifikant häufig dieselben Episoden als critical incidents bezeichnen (De Haan \& Nieß, 2018, S. 58), und die Autoren fordern, diesen Episoden im Rahmen der Coachingforschung besondere Aufmerksamkeit zu widmen (De Haan \& Nieß, 2012, S. 200).

Die Erkenntnisse von De Haan \& Nieß (ebd.) finden eine Parallele im generischen Modell der Psychotherapie (Orlinski, Ronnestad \& Willutzki, 2013, S. 52ff), das verschiedene Prozessfacetten beschreibt, unter anderem auch einen klinischen Aspekt im Sinn einer In-Session-Veränderung. Gemeint sind damit

„Geschehnisse wie Einsicht, Katharsis, die Lösung intrapersonaler Konflikte, Erfahrungen von Selbstwirksamkeit beim Problemlösen und verstärkte Gefühle von Hoffnung und Entschlossenheit weiterzumachen.“(ebd., S. 522)

In ihren Untersuchungen konnten Orlinski et al (2013, S. 581) eine konsistente Korrelation zwischen solchen In-Session-Outcomes in Form unmittelbarer und mittelbarer Veränderung und positiven Outcomes im Sinn finaler Veränderung aufzeigen.

Zur Erforschung von In-Session-Veränderungen soll hier auf den von Greenberg (Rice \& Greenberg, 1984; Greenberg, 1986) postulierten Change-Events-Approach zurückgegriffen werden, der Aspekte des Sequential-Process-Design bzw. Microanalytic-Sequential-Design mit dem Significant-Events-Approach verbindet. Als Change Events werden dabei diejenigen Abschnitte bzw. Episoden im therapeutischen Prozess bezeichnet, die durch eine unmittelbare 
Veränderungswirkung als Ergebnis intraindividuellen und interaktionellen Geschehens gekennzeichnet sind und drei relevante Komponenten enthalten (Greenberg, 2007, S. 16):

1. eine Problembeschreibung (problem marker) durch den Klienten

2. eine Performanz des Klienten im Sinn des Arbeitens am benannten Thema (the task), meist in Verbindung mit einer (Sprech-) Handlung des Therapeuten

3. eine unmittelbare Wirkung oder Veränderung als Ergebnis der vorhergehenden Schritte (resolution).

Auch Elliot (1984, S. 251) betont den Nutzen einer solchen auf signifikante Change Events ausgerichteten Forschung, weil damit tatsächlich besonders relevante „critical incidents“ oder „turning points“ in Abgrenzung zu einer sonst häufig anzutreffenden an Mittelwerten orientierten Vorgehensweise untersucht werden können. Allerdings weist er darauf hin, dass solche Events zum einen eher selten sind und zum anderen hoch komplex und daher sehr spezifisch und gründlich untersucht werden sollten. Dabei stellt sich auch die Frage, wie besonders relevante Change Events von solchen unterschieden werden können, die weniger bedeutsam für den Gesamtprozess sind. Gendlin (1986) und Benecke (2014, S. 627) weisen darauf hin, dass diese Frage gerade vor dem Hintergrund der angenommenen Nicht-Linearität von Veränderungsprozessen ein grundlegendes Problem der Prozessforschung darstellt, so dass die Selektion nach klaren Kriterien erfolgen muss:

„The subprocess is often defined only by type. One assumes it is always done well. Instead, let us define how it is done when it is effective. Then we can also study how therapists engender or participate in these differences." (Gendlin, 1986)

\subsection{Qualitative Task Analyse}

Die in diesem Vorhaben formulierten Prozessfragen gehen von theoretischem Vorwissen zum Coaching, zu Veränderungsmomenten und zum Experiencing aus, so dass eine rein induktive Analyse des Datenmaterials den Fragen nicht gerecht werden würde. Gleichzeitig aber würde eine Begrenzung auf eine theoriegeleitete Ex-ante-Kategorienbildung und eine rein deduktive Vorgehensweise zu einem geschlossenen Untersuchungssystem führen, das zu wenig offen wäre für das Entdecken neuer Ausprägungen und damit dem Interesse, durch Neuentdeckungen zur Weiterentwicklung von Theorie und Praxis beizutragen, sehr wahrscheinlich nicht gerecht werden würde.

Die von Rice, Greenberg und Pascual-Leone (Greenberg, 2007, Rice \& Greenberg, 1984, Pascual-Leone et al, 2009) entwickelte Methode der Task Analyse schlägt hier einen Weg ein, der geeignet scheint, theoriegeleitet und gleichzeitig offen für Neuentdeckungen vorzugehen. Während diese Forschungsmethode in der Arbeitspsychologie ursprünglich der Analyse von Kompetenzen, die eine bestimmte arbeitsbezogene Aufgabe erforderte, diente, wurde sie später auch eingesetzt, um kognitive Problemlöseprozesse besser zu verstehen und psychotherapeutische Veränderungsmomente zu erforschen (Greenberg, 1984b, S. 129, zitiert 
nach Newell \& Simon, 1972).

„Eine weitere Innovation im Bereich der Prozessforschung, die sich als bemerkenswert fruchtbar erwiesen hat, war die Einführung der ,aufgabenanalytischen“ Methode zur intensiven Analyse von Therapiesitzungen durch Rice und Greenberg (1984), bei der die Indikatoren für bedeutsames therapeutisches Geschehen, sogenannte „Marker“ auf eine Weise definiert werden, die es erlaubt, die Entwicklung und Auswirkung jenes Geschehens nachzuverfolgen und zu bewerten." (Orlinksi et al, 2013, S. 503)

Im Rahmen der Coachingforschung hat sich zuletzt Wegener (2019) mit der Frage nach bedeutsamen Sequenzen des Prozessgeschehens auseinandergesetzt. Sein Konzept der „,bedeutsamen Momente im Coaching“ geht zurück auf die Critical-Moments-Forschung von De Haan \& Nieß (2018) und auf den gesprächsanalytischen Coaching-Forschungsansatz von Graf $(2015,2018)$. Es geht davon aus, dass Veränderung im Coaching dann geschieht, wenn Coachees psychologische Wirkungen erleben, bei denen sich für sie etwas zum Positiven wendet und bei denen sie mentale Veränderungsprozesse im Sinn der Ausbildung funktionaler Deutungs- oder Handlungsmuster durchlaufen (Wegener, 2019, S. 98). Dem entsprechend zeigt sich Veränderung als sequentielles Geschehen, das sich aus der erfolgreichen Bewältigung von Teilschritten, die in einem engen Zusammenhang mit dem übergeordneten Coachinganliegen stehen, zusammensetzt (ebd., S. 185). Die Erwartung allerdings, dass besondere Veränderungsmomente in einem engen Zusammenhang zum übergeordneten Coachinganliegen und dem damit verbundenen Coachingziel stehen sollen, muss vor dem Hintergrund der Critical-Incidents-Forschung und vor dem Hintergrund einer prozessorientierten Sicht insofern relativiert werden, als dass nicht jederzeit ein direkter oder kausaler thematischer Zusammenhang zwischen mittelbarer Veränderung im Subprozess und finaler Veränderung im Gesamtprozess erkennbar sein muss. Vielmehr sind gelungene Subprozesse aus prozessorientierter Sicht als Teil eines insgesamt vorwärtstragenden Prozesses zu sehen, der nicht linear verlaufen muss und dessen Detailziele sich auch im Lauf des Prozesses selbst verändern können.

In der traditionellen Significant-Events-Forschung bzw. Critical-Events-Forschung werden bedeutsame Veränderungsmomente in der Regel durch die nachträgliche Einschätzung der Beteiligten bestimmt (De Haan \& Nieß, 2018, S. 57; Elliot, 1984, S. 252) und unterliegen damit einer unter Umständen retrospektiv und subjektiv eingefärbten Wahrnehmung, die nicht zwangsläufig identisch sein muss mit dem tatsächlich beobachtbaren Kommunikationsgeschehen im Coaching. Die Task Analyse schlägt hier einen anderen Weg ein, indem sie die in einer jeweiligen Gesprächsepisode erkennbare Aufgabenbewältigung mit einem vorab entwickelten Aufgabenmodell abgleicht. Damit macht sie sich unabhängig von individuellen Einschätzungen der Beteiligten und kann sich direkt auf ihr faktisches Kommunikationsverhalten und ihre sonstigen Verhaltensweisen stützen (Greenberg, 2007, S. 25), um bedeutsame Momente im Coaching zu identifizieren und zu analysieren. Bezogen auf die Change Process 
Research ordnet Wegener (2019, S. 82) dieses Vorgehen den am Significant-Events-Design orientierten Studien zu, deren primäres Ziel das Erforschen von bedeutsamen Veränderungsmomenten ist.

Die Task Analyse erinnert in Teilen an die qualitative Einzelfallstudie, die nach Döring \& Bortz (2016, S. 215) und Thoma \& Kächele (2006, S. 8) geeignet ist, durch genaues Erforschen und Beschreiben Fragestellungen über individuelle Prozesse und Verläufe zu beantworten und daraus auch allgemeine Aussagen, Ideen und Hypothesen zum Untersuchungsfeld abzuleiten. Stiles (2007) beschreibt dazu ein abduktives Vorgehen, das anhand einer gezielten Untersuchung des Einzelfalls der Weiterentwicklung eines Theoriemodells dienen soll. Im Unterschied dazu gilt das Interesse der Task Analyse allerdings nicht einer umfassenden Falluntersuchung und einer daraus abzuleitenden generellen Theoriebildung, sondern vielmehr gezielt der Bearbeitung und Erledigung der für die Forschungsfrage relevanten psychologischen Aufgabe im Sinn einer In-Session-Veränderung.

Auch zur Grounded Theory (Strauss \& Corbin, 1990) finden sich Parallelen, nicht nur hinsichtlich des Sampling, sondern auch bei der Durchführung der empirischen Analyse, bei der die Daten systematisch analysiert und mit theoretischen Vorannahmen abgeglichen werden. Die Besonderheit der Task Analyse besteht im Unterschied zur Grounded Theory darin, dass sie neben den bewussten Aussagen und Verhaltensweisen der Beteiligten auch solche Aspekte berücksichtigt, die zwar vom Forscher beobachtbar sind, derer sich die Beteiligten im Moment des Geschehens aber möglicherweise nicht bewusst sind (Pascual-Leone et al, 2009, S. $530)$.

Der spezifische Nutzen der Task Analyse ist darin zu sehen, dass sie detaillierte Beschreibungen von Veränderungsschritten und einen Abgleich zwischen einem theoretisch entwickelten Modell und dem tatsächlichen Klientenverhalten im Kontext der Coach-Coachee-Interaktion ermöglicht (Rice \& Saperia, 1984, S. 30, Rice \& Greenberg, 1984, S. 13). Um diese Veränderungsmomente auch wirklich beschreiben und verstehen zu können, fordern die Autoren von der Forschung, sich stärker auf die klientenseitigen Veränderungsmechanismen zu konzentrierten als auf das Therapeuten- oder Coachverhalten. Als Veränderungsmechanismen gelten zum einen der beobachtbare Prozess im Sinn des tatsächlichen Klientenverhaltens während der Sitzung, und zum anderen der mentale und Information verarbeitende Prozess des Klienten. Dies erfordert, ausgehend vom beobachtbaren Prozess, auch Rückschlüsse auf die internalen Operationen des Klienten zu ziehen. Während andere Forschungsmethoden eher technische oder interaktionelle Aspekte des generischen Prozessmodells (Orlinski, 2013) in den Blick nehmen, versucht die Task Analyse vor allem, intrapersonale Prozesse bzw. die im Modell als klinischer Aspekt bezeichneten In-Session-Impacts ins Zentrum der Untersuchung zu rücken. Dies setzt zum einen eine „klinische Informiertheit“ des Forschers voraus (Rice \& Greenberg, 1984, S. 20), und zum anderen eine ,theoretisch-empirische“ Herangehensweise (ebd., S. 19), da sich intrapersonale Prozesse nur als Abgleich zwischen theoretischen Überlegungen und dem in der Episode beobachteten Verhalten beschreiben lassen. 
In der Forschungspraxis geht die Task Analyse in der Regel zunächst von einer generellen Theorie aus - in diesem spezifischen Fall von einer Veränderungstheorie und daraus abzuleitenden Prozessen, um dann durch differenzierte Untersuchungen des empirischen Materials zur weiteren Theoriebildung zu gelangen. So empfehlen Rice \& Saperia (1984, S. 31):

„The investigator may begin with an explicit general theory of therapy and personality development, as well as a kind of implicit clinician's cognitive map concerning the important interactions of therapy. In other words, one approaches the study of therapeutic events with a great deal of clinical wisdom based on theory and observation."

Das Gesamtkonzept der Task Analyse sieht ein zweiphasiges Vorgehen vor: In der ersten, qualitativ orientierten Phase steht das Interesse, Neues zu entdecken im Vordergrund, während die zweite, quantitativ orientierte Phase dem Testen und der Validierung der erstellten Hypothesen dient (Greenberg, 2007, S. 15). Dadurch wird die Task Analyse insgesamt zu einem aufwändigen multimethodischen Verfahren. Die in der ersten Phase gewonnenen Erkenntnisse können erst dann als bestätigt gelten, wenn sie durch eine quantitative Untersuchung anhand einer neuen Datenbasis abgesichert wurden. Gleichwohl weisen verschiedene Autoren (Pascual-Leone et al, 2009, S. 530, Elliot, 2012a, S. 73) darauf hin, dass je nach Zielsetzung und Fragestellung auch eine Beschränkung auf eine der beiden Phasen der Task Analyse möglich ist. Insbesondere Elliot (ebd.) betont den Nutzen der qualitativen Analyse für die Entwicklung oder Weiterentwicklung eines theoretischen Modells. Dazu beschreibt er folgende Elemente der Task Analyse (ebd., S. 74):

- Beschreiben eines theoretischen Modells (rational model), das den Veränderungsprozess und die damit in Verbindung stehenden Aufgaben beschreibt

- Identifikation von gelungenen Veränderungsprozessen bzw. von Episoden, in denen die Aufgabe erfolgreich bewältigt wurde

- Ausführliche qualitative Beschreibung und Analyse der betreffenden Episoden auf Basis des empirischen Materials

- Abgleich der qualitativen Analyse mit dem theoretischen Modell und Weiterentwickeln des theoretischen Modells vor dem Hintergrund der im Rahmen der Analyse gewonnenen Erkenntnisse

- Weitere Untersuchung des revidierten Aufgabenmodells anhand weiterer Change Events.

Ziel dieses dialektischen Abgleichens zwischen Theorie und empirischer Beobachtung ist die Entwicklung oder Weiterentwicklung eines rational-empirischen Prozessmodells. Dies deckt sich mit der Intention dieser Studie, den Prozess der Veränderung im Coaching bzw. im Kontext des Experiencing besser zu verstehen.

Mit Blick auf die spezielle Fragestellung dieser Arbeit wird bei der Umsetzung der Task Analyse darauf zu achten sein, dass hier nicht primär die Theorieentwicklung zum Experiencing 
im Vordergrund steht, sondern die Frage nach coachingspezifischen Besonderheiten. Im Kontext der Therapieforschung sind Experiencingprozesse umfangreich beforscht, so dass die Erwartung, die Experiencing-Theorie generell weiterzuentwickeln, nicht angebracht scheint. Im Kontext von Coaching sind Experiencingprozesse allerdings noch wenig untersucht, so dass sich hier folgende Fragestellungen von Interesse sind:

- Wie lässt sich ein im Rahmen von Coaching stattfindender Experiencingprozess beschreiben - auch dann, wenn das Coaching nicht explizit nach der FocusingMethode durchgeführt wird?

- Was trägt zu einem gelingenden Subprozess bei?

- Wo sind „Bruchstellen“ erkennbar, also Muster, die zu einem Abbruch und damit zu einer weniger erfolgreichen Aufgabenbewältigung führen?

Diesen Fragen lässt sich idealerweise im Rahmen der qualitativen, entdeckenden Phase der Task Analyse nachgehen. Entsprechend soll sich die Task Analyse hier auf die qualitative Phase begrenzen.

Greenberg (1996; 2007, S. 16) und Pascual-Leone et al (2009) konkretisieren die methodische Umsetzung der Task Analyse anhand mehrerer aufeinander aufbauender Schritte, die im Folgenden dargestellt werden sollen. Dabei ist allerdings zu berücksichtigen, dass die lineare Abfolge der einzelnen Schritte in der Praxis nicht immer einzuhalten ist, so dass im Forschungsprozess auch ein rekursives Vorgehen indiziert sein kann, bei dem vorhergehende Analyseschritte nochmals anhand der Erkenntnisse nachfolgender Analyseschritte überprüft und nachbereitet werden (Gollino, 2016, S. 86). Üblich ist auch eine Vorab-Analyse im Sinn einer Sichtung der vorhandenen Gesprächsprozesse und der Skizzierung eines provisorischen Modells, um die Task Marker auch angemessen beschreiben zu können (vgl. Gollino, 2016, S. 88, Safran, Muran \& Samstag, 1994, S. 227).

Wenn im Rahmen der Task Analyse vom theoretischen Modell die Rede ist, ist noch auf eine in der deutschen Sprache auftauchende begriffliche Unschärfe hinzuweisen: Gemeint ist damit nicht die zugrundeliegende psychologisch-theoretische Konzeption, sondern ein aus dem theoretischen Vorwissen abgeleitetes Ablaufmodell des Veränderungsprozesses. Im englischen Original wird hierfür der Begriff ,rational model“" benutzt, während das aus der empirischen Analyse heraus entwickelte Ablaufmodell als „empirical model“ bezeichnet wird.

\subsubsection{Spezifizieren der Aufgabe}

Veränderungsprozesse werden in der Change Events Forschung als solche Prozesse betrachtet, bei denen der Klient eine psychologische Aufgabe bewältigt, die zur Lösung eines von ihm erlebten ,affektiv-kognitiven Problems“ (Wegener, 2019, S. 83) bzw. zu einer Veränderung bezüglich des thematisierten Problems führt. Im ersten Schritt der Task Analyse ist daher die Aufgabe, die für die Fragestellung des Forschungsprojekts relevant ist und die untersucht werden soll, zu spezifizieren. In weiteren Schritten können dann die Komponenten der Veränderung und schließlich die Wirkung der Veränderung (Lösung) näher beschrieben werden. 
Um eine angemessene Task Analyse durchführen zu können, soll die Aufgabe konkret formuliert werden und solche Verhaltensaspekte beinhalten, die anhand der Audiographien bzw. Transkripte beobachtbar sind. Sie soll folgende Kriterien erfüllen:

- Die Aufgabe soll eine gewisse Bedeutung für den Coachingprozess haben

- Wenn die Aufgabe erfolgreich bewältigt ist, soll ein Aspekt der Veränderung erkennbar sein

- Der Klient soll, um die Aufgabe zu erledigen, für den Forscher beobachtbare Handlungen vollziehen

- Der Klient soll die Aufgabe innerhalb eines definierten Kontextes, hier als Aufgabenumgebung bezeichnet, bearbeiten

- Der Beginn und das Ende der Aufgabe soll für den Forscher identifizierbar sein. Daher sollen Marker für den Beginn der Aufgabe und für die erfolgreiche Aufgabenbewältigung beschrieben werden. Die Marker müssen allerdings nicht vom Klienten oder dem Coach als solche intendiert oder erkannt sein.

Das schrittweise Vorgehen sieht vor, zunächst das zu Verfügung stehende Material zu sichten und im Material (Audiographien und Trankskripte) drei Beispiele einer im Coaching beobachtbaren Verhaltensweise des Klienten auszuwählen, die aus der Sicht des therapeutisch erfahrenen Forschers typische Beispiele der relevanten Aufgabe darstellen. Ergänzend werden als Kontrast drei weitere Beispiele ausgewählt, bei denen der Klient sich nicht in diesem Aufgabenbereich befindet. Die Beispiele dienen als Grundlage, um Erkennungsmerkmale und Merkmale zur Abgrenzung der Aufgabe zu beschreiben. Nachdem auf diese Weise ein TaskMarker initial beschrieben ist, werden im Material weitere Beispiele für die Aufgabe identifiziert und beschrieben, bis eine gewisse Sättigung eingetreten ist, bis also weitere Beispiele keine neuen Informationen zur Spezifizierung der Aufgabe mehr hervorbringen würden.

\subsubsection{Erklären der kognitiven Karte}

Nach Hein \& Sewz (2005, S. 10) ist die arbeitspsychologische Forschung immer auch vor dem Hintergrund epistemologischer Grundlagen zu betrachten. Auch Greenberg (2007) fordert vom Forschenden, die eigene kognitive Landkarte, anhand derer er sich im Forschungsfeld bewegt, möglichst klar zu benennen. Der von Greenberg (ebd.) verwendete englischsprachige Terminus der „cognitive map“ (vgl. Tolman, 1948) beschreibt die mental vereinfachte Repräsentation komplexer Zusammenhänge und Realitäten und wird oft mit „Mentales Modell" in die deutsche Sprache übertragen, wobei die Termini je nach Autor und Kontext auch unterschiedliche Verwendung finden (Jones \& Bouncken, 2008, S. 736).

Bezogen auf die Task Analyse, geht es in diesem Schritt darum, anzuerkennen, dass Mentale Modelle im Sinn kognitiver Landkarten Einfluss auf die Wahrnehmung des Forschenden und damit auch auf die theoretische und empirische Erarbeitung eines Themas haben. Mit der Spezifizierung der eigenen kognitiven Karte wird diese expliziert, um so einer Verzerrung 
der Forschung durch sonst nur implizit vorhandene Vorstellungen entgegenzuwirken und damit die Objektivität der Forschung zu wahren. So sollen in diesem Forschungsschritt konkret die Vorannahmen, Sichtweisen und Thesen, mit denen der Forscher dem Material begegnet und die sowohl seine Herangehensweise als auch die weitere theoretische und empirische Erarbeitung des Themas beeinflussen, zusammengefasst werden.

Auch hier zeigen sich Parallelen zur Grounded Theory bzw. dem der Grounded Theory Methodoloy zugrunde liegenden hermeneutischen Verständnis:

„Im Konzept des hermeneutischen Verstehens wird davon ausgegangen, dass man (...) bei der Konfrontation mit einem Phänomen (...) bereits mit einem gewissen Vorverständnis daher kommt (...). Eine solche apriorische Annahme leitet unser Verstehen / Deuten des Phänomens zu einem bestimmten Zeitpunkt (...). Beim Verstehensakt, bei der Verarbeitung von Wahrnehmung und Deutung, wird dieses (Vor)Verständnis angereichert - und es stellt dann einen erweiterten Annahmehintergrund für den nächsten Verstehensakt dar.“ (Breuer, Dieris \& Lettau, 2009, S. 48)

Die Task Analyse folgt diesem spiralförmigen Verständnis von Erkenntnisgewinn im Sinn eines hermeneutischen Zirkels, nach dem die wechselseitige Einflussnahme von Theorie-Verständnis und Ereignis-Deutung zum vertieften Verstehen führen.

\subsubsection{Spezifizieren der Rahmenbedingungen}

Hier gilt es, die Aufgabenumgebung bzw. den Kontext zu definieren, der für die Erledigung der Aufgabe gelten soll. Damit wird neben dem Task Marker ein weiteres Kriterium für das Sampling eingeführt: nur dann, wenn dieser Kontext gegeben ist, soll die entsprechende Sequenz zur weiteren Untersuchung herangezogen werden.

Zum einen ermöglicht dieser Schritt dem Forscher, den zu erforschenden aufgabenbezogenen Prozess einschließlich des Prozess-Endes näher einzugrenzen. Zum anderen kann der Forscher so einen Rahmen schaffen, auf dessen Grundlage er sich dann auch tatsächlich auf die Verhaltensvariationen des Klienten beim Lösen seines Problems bzw. seiner Aufgabe konzentrieren kann, ohne in jedem Einzelfall den Einfluss individueller Rahmenbedingungen berücksichtigen zu müssen. Zudem können die Kontextbedingungen aufgrund des Vorverständnisses zum Forschungsthema so festgelegt werden, dass ein Rahmen für eine möglichst gelingende Aufgabenbewältigung gegeben ist (vgl. Rice \& Saperia, 1984, S. 31, Greenberg, 1984, S. 71).

Häufig wird der für die jeweilige Task Erledigung bzw. Task Analyse vorausgesetzte Kontext in Form eines kurzen Manuals dargestellt, das die Interventionen des Therapeuten oder Coachs beschreibt, die dem Klienten bei der Aufgabenbewältigung helfen sollen. Ein solches Manual ist vor allem dann hilfreich, wenn die Aufgabenerledigung vor dem Hintergrund einer bestimmten methodischen Vorgabe für den Coach untersucht werden soll und wenn die jeweilige Aufgabe für den Coach explizit erkennbar ist. In Situationen allerdings, in denen die 
Aufgabe von den Beteiligten nicht immer klar als solche erkannt werden kann, sondern vielmehr implizit vorhanden ist, empfehlen insbesondere Pascual-Leone et al (2009, S. 532), die Aufgabenumgebung eher weit gefasst zu beschreiben und anstelle der Interventionen des Coachs stärker auf diejenigen Kontextmerkmale oder Marker abzuheben, die den Beginn der Aufgabe bzw. ihrer Bearbeitung und das Ende der Aufgabenerledigung kennzeichnen.

\subsubsection{Bilden eines theoretischen Aufgabenmodells}

Nachdem das Problem und die Aufgabenumgebung beschrieben sind, werden in diesem Schritt auf der Basis bisheriger Erkenntnisse und theoretischer Vorannahmen Hypothesen zur Aufgabenbewältigung gebildet. Der Forscher beschreibt seine Thesen, wie das Problem in dieser Aufgabenumgebung gelöst werden könnte, im Rahmen eines theoretischen Modells, das später am Fallmaterial überprüft werden kann. Das Modell wird aus klinischer bzw. wissenschaftlicher Sicht gebildet, die Leitfrage dabei ist: Wie lösen Klienten nach Meinung des Forschers diese spezielle Aufgabe? Die Antwort darauf leitet sich ab aus der Theorie und dem Problemlöseverständnis, das der Forscher aufgrund seiner dargelegten kognitiven Landkarte hat. Es präzisiert die Annahmen des Forschers zur Aufgabenbewältigung und beschreibt den vermutlichen Prozess, wie er im Idealfall stattfinden könnte.

Praktisch wird das theoretische Aufgabenmodell in Form eines Ablaufdiagramms erstellt, das später als „Baseline“ dient und einen Abgleich mit den empirischen Entdeckungen ermöglicht.

\subsubsection{Durchführen der empirischen Analyse}

Der Forscher untersucht in diesem Schritt, der als das Herzstück der entdeckungsorientierten Phase der Task Analyse gilt, den aktuellen In-Session-Veränderungsprozess des Klienten. Er versucht dabei, sein eigenes Vorwissen so gut es geht beiseite zu stellen, ebenso seine Annahmen, Erwartungen etc., die er im theoretischen Modell expliziert hat. Primäre Aufgabe ist, die tatsächliche Performanz so weit als möglich zu erfassen.

Ziel der empirischen Analyse ist ein mittels einer qualitativen Untersuchung erarbeitetes empirisches Modell. Durch Beobachtung sollen die zentralen Komponenten der Aufgabenerledigung bzw. des Veränderungsprozesses, ausgehend vom Task Marker bis hin zur Erledigung bzw. Lösung der Task, erfasst werden. Im Gegensatz zur theoretischen Modellbildung nimmt der Forscher in der empirischen Analyse die Haltung des Skeptikers ein und sucht bewusst nach Differenzen zwischen dem Theoriemodell und dem empirischen Befund, um so theoretische Annahmen zu widerlegen und dadurch das Theoriemodell weiterzuentwickeln (Pascual-Leone et al, 2009, S. 534). Das Vorgehen entspricht dem der Abduktion: Ausgangspunkt ist eine a priori formulierte Theorie, anhand derer der Veränderungsprozess beobachtet wird (Greisel, 2015, S. 173). Die abduktive Forschungsfrage ist nun die, ob es sich beim beobachteten Prozess tatsächlich um einen Anwendungsfall des Theoriemodells handelt bzw. inwieweit das theoretische Modell aufgrund des empirischen Befunds angepasst werden muss.

Die Analyse soll in zwei Etappen erfolgen. In jeder der beiden Etappen wird das empirische 
Material zunächst auf der Grundlage des theoretischen Modells codiert und kategorisiert, und in einem zweiten Schritt wird nach weiteren Kategorien im Material gesucht, die dann ebenfalls codiert werden. Ergänzend wird überprüft, ob die theoriebasierten Codes auch wirklich relevante Merkmale und Unterschiede abbilden, und bei Bedarf werden dieses Codes an den empirischen Befund angepasst. So entsteht ein mehrschrittiger Prozess der Kategorienbildung, der zwei Zwecke hat: Zum einen wird das Denken des Forschers geschärft, und zwar darüber, wie der empirische Befund das theoretische Modell entweder bestätigt oder verändert. Zweitens kann das Vorgehen neue Kategorien hervorbringen, die jeweils in der untersuchten Task bzw. im beforschten Change Event eine spezifische Bedeutung haben können. Konkret empfehlen Rice \& Saperia (1984, S. 40), in der ersten Etappe zunächst drei „ideale“ Subprozesse zu analysieren und das theoretische Modell auf Grundlage der empirischen Erkenntnisse aus diesen drei Prozessen weiterzuentwickeln. In einer zweiten Etappe können dann drei weitere Prozesse ausgewählt werden, anhand derer das weiterentwickelte Modell wiederum überprüft und modifiziert wird. Das entsprechende Vorgehen kann in folgenden Schritten dargestellt werden:

1. Gezieltes Sampling: aus der Grundgesamtheit der zur Verfügung stehenden Coachingprozesse werden diejenigen Subprozesse für die weitere Untersuchung ausgewählt, bei der die Bearbeitung relevanter Teilaufgaben als ideal identifiziert werden kann. Für die Stichprobe wird analog zu Rice \& Saperia (1984) N = 6 angestrebt, aufgeteilt in zwei Halbgruppen für die beiden Etappen

2. Etappe 1: Codierung des vorliegenden Materials der ersten Sample-Halbgruppe auf Grundlage des theoretischen Modells

3. Suche nach weiteren Codes und Überprüfung der vorhandenen Codes. Gegebenenfalls Bildung weiterer Codes und Anpassung der bisherigen Codes

4. Etappe 2: Die Schritte 2 und 3 werden nochmals durchgeführt, Basis hierfür ist die zweite Sample-Halbgruppe

5. Rekonstruktion eines ,client operating model“, das die Veränderungsmechanismen und das Experiencing des Klienten möglichst präzise beschreibt (Rice \& Saperia, 1984, S. 58).

Während Rice \& Saperia (ebd.) auf die Kontrastierung der Fälle verzichten, wird an anderer Stelle bei einer Stichprobe von $\mathrm{N}=6$ auch empfohlen, die Untersuchung zunächst mit drei erfolgreichen Sequenzen zu beginnen, die sowohl den Task Marker beinhalten als auch ein gelungenes Ende der Aufgabenerledigung, und die zweite Etappe der Untersuchung dann für eine Kontrastierung zu nutzen, also als Vergleich drei weniger erfolgreiche Sequenzen der Aufgabenerledigung heranzuziehen (Pascual-Leone, 2009, S. 534, vgl. Schütze, Fiedler \& Krüger, 2016, S. 31). Letzteres Vorgehen ermöglicht, die tatsächlich optimalen Prozesse der Veränderung gegen weniger optimale Prozesse abzugrenzen. Gerade wenn es darum geht, auch Abbruchmuster im Prozess zu identifizieren, scheint es geradezu geboten, die Fälle zu kontrastieren. 
Beim Codieren geht es darum, beobachtete Phänomene in Bezug zu bringen zu Begriffen, Ideen und Konzepten, auf die sie verweisen oder mit denen sie in einem gewissen Zusammenhang stehen (Breuer et al, 2009, S. 51). So können Konturen des Prozesses herausgearbeitet werden, die dann in die weitere Modellbildung zur Forschungsfrage einfließen. Das Codieren im Rahmen der Task Analyse erfolgt in mehreren Durchgängen und soll verschiedene Aspekte erfassen (Rice \& Saperia, 1984, S. 58, Greenberg, 2007, S. 20):

- Zunächst werden aus dem theoretischen Modell Codes abgeleitet und auf den zu untersuchenden Subprozess angewendet

- Für die Suche nach weiteren Codes werden dann zwei oder drei Makrokategorien gebildet, die ermöglichen sollen, verschiedene Stränge des Veränderungsprozesses abzubilden und anschließend miteinander zu vergleichen

- Die weitere Codierung verläuft entlang dieser Makrokategorien: hier werden zunächst „Lower-Level-Kategorien“ gebildet, also solche Codes, die sich nah am Kommunikationsgeschehen orientieren. In einem weiteren Schritt werden die „Lower-Level-Kategorien“ nochmals überprüft und gegebenenfalls „Higher-Level-Kategorien“" notiert, um ein größeres Abstrahierungsniveau zu erreichen.

Ergänzend sollen bei der empirischen Analyse auch standardisierte Beobachtungsinstrumente wie beispielsweise die EXP-Skala zum Einsatz kommen (Greenberg, 2007, S. 22). Diese Beobachtungsinstrumente sind zum einen für eine spätere quantitative Validierung von Bedeutung. Aber auch wenn diese nicht vorgesehen ist, dient ein Abgleich der Codes mit den Maßen der Beobachtungsskala auch in der entdeckenden Phase der Task Analyse dem Erkenntnisgewinn.

Im Anschluss an die Codierung erfolgt eine Verdichtung des Materials: Passagen und Aspekte, die nicht direkt Einfluss auf den taskbezogenen Veränderungsprozess nehmen, werden beiseite gelegt, so dass die zentralen Veränderungsschritte deutlicher erkennbar werden. 


\subsubsection{Erstellen eines theoretisch-empirischen Modells}

Hier geht es darum, aus dem primären theoretischen Modell und den empirischen Befunden eine Synthese zu erstellen und daraus ein modifiziertes theoretisches Modell abzuleiten. Ziel ist, die tatsächlichen Veränderungsprozesse der Klienten möglichst genau wiederzugeben, so dass eine theoretisch-empirische Skizze des Lösungsprozesses entsteht.

Dazu wird zunächst aus den empirischen Befunden jedes einzelnen Fallbeispiels - sowohl der idealtypischen Beispiele als auch der kontrastierenden Beispiele - ein Modell in Form eines Diagramms abgeleitet. Die einzelnen empirischen Modelle werden dann jeweils untereinander und mit dem theoretischen Modell verglichen und in ein gemeinsames theoretisch-empirisches Modell überführt, das das „,cumulative understanding of these comparisons“ (Aspland, 2008, S. 706) des Forschenden abbildet.

Die Codierung des Materials und das Erstellen der einzelnen empirischen Modelle sind in der Praxis der Forschung zwei eng miteinander verwobene Schritte, deren Herausforderung darin besteht,

„to sort and to find invariant patterns (i.e., patterns recurrent across types of situation) in the essential task performances that contribute task completion. In the end, the qualitative approach that is necessary here is to identify and elaborate essential aspects of the phenomena's teleological structure until such a model reaches stability. Thus, as the data are empirically modeled, they inform the rational model such that a synthesis of rational and empirical models (...) is created. This is done by adding, modifying, or deleting rationally based model components in accordance with empirical observations and by similarly accommodating empirically observed steps within the model." (Pascual-Leone et al, 2009, S. 534)

\subsubsection{Theoriebasiertes Erklären des modizifierten Modells}

Während das theoretisch-empirische Modell zunächst die Handlungen bei der Aufgabenbewältigung bzw. im Veränderungsprozess des Klienten beschreibt, geht es hier darum, die Handlungen auch zu erklären und theoretisch zu begründen.

\section{$5.4 \quad$ Theoriegeleitetes Sampling}

Analog zum Vorgehen der Grounded Theory (Strauss \& Corbin, 1990) wird im Rahmen der Task Analyse bei der Auswahl der zu untersuchenden Fälle in der Regel ein gezieltes theoriegeleitetes Sampling vorgenommen, um einen möglichst hohen Erkenntnisgewinn bezüglich der zugrundeliegenden theoretischen Überlegungen zu erhalten.

„Sampling bedeutet in qualitativer Forschung die Ziehung derjenigen Subjekte, die sich als inhaltlich adäquat im Hinblick auf die Forschungsfrage erweisen und die reichhaltige Informationen zu dieser zu liefern versprechen.“(Misoch, 2015, S. 186)

So wählten beispielsweise Rice \& Saperia (1984) in ihrer auf der klientenzentrierten Theorie 
basierenden Untersuchung Fälle aus dem Bereich der klientenzentrierten Therapie, und Greenberg (Greenberg, 1984a) untersuchte in seiner Task Analyse zur intrapersonalen Konfliktlösung gezielt gestalttherapeutisch ausgerichtete Prozesse. Auch andere Auswahlkriterien für die Sample-Bildung sind denkbar, solange sie im Zusammenhang mit der Forschungsfrage stehen und zu erwarten ist, dass Fälle oder Daten, die diesen Kriterien entsprechen auch einen erweiternden oder ausdifferenzierenden Beitrag zur Beantwortung der Forschungsfrage leisten könnten. Nach Misoch (2015, S. 194, zitiert nach Patton, 1990) sollen aus der Grundgesamtheit der für die Analyse zur Verfügung stehenden Prozesse diejenigen Episoden auswählt werden, die dem Auswahlkriterium am meisten entsprechen. Die in anderen Designs relevanten Kriterien der Repräsentativität oder der Zufallslogik spielen hier eine untergeordnete Rolle.

Im Unterschied zum schrittweisen theoretischen Sampling, wie es häufig im Rahmen der Grounded Theory Methode zu Einsatz kommt (Breuer et al, 2009, S. 41), findet die Samplebildung im Rahmen der Task Analyse allerdings nicht Zug um Zug statt, vielmehr ermöglichen die theoretischen Vorüberlegungen dem Forscher, die zur Analyse kommenden Fallbeispiele in einem Durchgang auf Grundlage der Task Marker und der spezifizierten Rahmenbedingungen auszuwählen.

Theoriegeleitetes Sampling im Rahmen der Task Analyse bedeutet also, diejenigen Episoden auszuwählen, die aufgrund des Vorverständnisses möglichst geeignet erscheinen, Aufschluss über die Art und Weise der Taskbearbeitung zu geben.

\subsection{Gütekriterien}

„Während in der Quantitativen Forschung die zentralen Gütekriterien für methodische Strenge sowie für die anderen Qualitätskriterien der Wissenschaft (...) relativ konsensfähig und in vielen Bereichen detailliert ausformuliert sind, wird die Debatte um geeignete Qualitätskriterien in der qualitativen Forschung sehr viel kontroverser geführt.“ (Döring \& Bortz, 2016, S.106)

Unstrittig ist, dass auch qualitative Forschung sich an den allgemeinen Kriterien der Reliabilität und der Validität orientieren (Mayring, 2007, S. 109) und vertrauenswürdig, übertragbar, zuverlässig und bestätigbar sein sollte (Döring \& Bortz, 2016, S. 108).

Wie die Umsetzung der Gütekriterien konkret ausgestaltet sein kann, ist Gegenstand vielfältiger Diskussionen und auch abhängig vom jeweiligen methodischen Vorgehen. Für die Task Analyse sind keine spezifisch formulierten Gütekriterien bekannt, so dass hier vor dem Hintergrund, dass das Vorgehen der Task Analyse Parallelen zur Grounded Theory aufweist, auf die Gütekriterien der Grounded Theory-Methodik zurückgegriffen werden soll. Unter Bezugnahme auf Steinke (2007) formulieren Breuer et al (2009) dazu folgende Kernpunkte:

- „Intersubjektive Nachvollziehbarkeit: Dokumentation des Forschungsprozesses, Interpretationen in Gruppen, Anwendung kodifizierter Verfahren 
- Indikation des Forschungsprozesses: Geeignetheit des qualitativen Vorgehens, Angemessenheit der Methodenwahl, der Transkriptionsregeln, der Samplingstrategie, der Einzelentscheidungen im Gesamtkontext, der Bewertungskriterien

- Empirische Verankerung: Verwendung kodifizierter Methoden, Aufweis von Textbelegen, Verwendung Analytischer Induktion, Ableitung prüfbarer Prognosen, kommunikative Validierung

- Limitation: Austesten bzw. Angabe der Grenzen des beanspruchten Geltungsbereichs durch Fallkontrastierung und Suche nach abweichenden Fällen

- Kohärenz: Konsistenz des Aussagesystems, Widerspruchsfreiheit

- Relevanz: praktischer Nutzen

- Reflektierte Subjektivität: Selbstbeobachtung des Forschers, Reflexion der Forschungsbeziehung." (ebd., S. 109)

$\mathrm{Zu}$ berücksichtigen ist insgesamt, dass qualitative Forschung nicht den Anspruch der Repräsentativität erhebt und auch nicht auf die zahlenmäßige Verteilung bestimmter Merkmale abzielt (Lamnek, 2005, S. 183). Insofern ist nicht der Anspruch zu erheben, mit einer Studie alle im Feld vorkommenden Muster zu erfassen. Andererseits soll es Ziel der Erhebung sein, „der Vielfältigkeit der Erfahrungen (...) Raum zu geben“ (Langer, 2000, S. 38), so dass möglichst unterschiedliche Fälle in die Untersuchung einbezogen werden sollen.

Wenn eine Studie auf Theorieentwicklung abzielt, verweisen Breuer et al (2009, S. 110) auf das Kriterium der theoretischen Sättigung: das zu entwickelnde Theoriemodell wird so lange mit Fällen und Daten abgeglichen, bis ein weiteres Hinzuziehen von Fällen oder Daten keinen weiteren Beitrag mehr zur Erweiterung oder Veränderung des Modells leisten würde. Die daraus resultierende „theoretische Sättigung“ (ebd., S. 58) ist allerdings als Idealpostulat zu verstehen, in der Praxis der Forschung ist eine diesermaßen umfassende Sampleerstellung selten umsetzbar.

Im Rahmen der Task Analyse fordert Greenberg als weiteres Kriterium (2007, S. 16):

"Because identification of key change events is of particular importance in conducting a task analysis, theoretical understanding and clinical experience in the therapeutic approach to be studied are generally required. Task analysis thus is best done by clinician-scientists who understand the therapeutic process they wish to study rather than by naïve observers or nonclinician researchers."

Auch Wegener (2019, S. 82) greift diese Forderung nach therapeutisch geschulten Wissenschaftlern auf, und es kann als ein Aspekt der Glaubwürdigkeit verstanden werden, wenn sowohl Theoriewissen als auch klinische Erfahrung in den Auswertungsprozess einfließen. Gleichzeitig könnte gerade der Einfluss von Vorwissen und Erfahrung als eine Verletzung der Neutralität gewertet werden. Hier weist Langer (2000, S. 66) darauf hin, dass generell die in der Forschung oft geforderte Objektivität und Neutralität bei qualitativen Methoden nur eingeschränkt gegeben sein kann. Die Beobachtungen des Forschers beruhen immer auf seinen Sinneseindrücken und seinen verstandesmäßigen Beurteilungen des Phänomens, die auch 
von seinen Vorerfahrungen und Fachkenntnissen geprägt sind. Sie können nicht ausgeblendet, wohl aber durch eine gründliche Explikation kontrolliert werden.

Ergänzend sollte ein besonderes Augenmerk auf die Codierung und das Rating von Daten gelegt werden. Codes und Ratings sollten reliabel, also stabil und replizierbar sein. Um dies zu überprüfen, wird eine Wiederholung der Analyse ohne Kenntnis der ersten Codes oder Ratings und mit einem zeitlichen Abstand empfohlen (Mayring, 2007, S. 112). 


\section{Durchführung der Studie}

In dieser Studie soll der intrapersonale experienzielle Prozess untersucht werden, der im Coaching zu einer Veränderung führt oder mit dieser einhergeht. Dabei stellt sich die Frage, inwieweit tatsächlich coachingspezifische Aspekte eines experienziellen Prozesses zu erkennen sind, die zu einer Weiterentwicklung des theoretischen Modells führen könnten, und ebenso stellt sich die Frage nach den Implikationen für die Coachingpraxis, also beispielsweise, wie ein experienzieller Prozess im Coaching gefördert werden kann, oder auch, an welchen Stellen es möglicherweise zu Prozessabbrüchen kommt.

Bei der Arbeit mit dem Material gab es immer wieder Augenfälligkeiten und Überlegungen des Autors, die dieser festhalten wollte. So wurde ein Forschungstagebuch angelegt, in dem entsprechende Memos, sofern sie nicht direkt im jeweiligen Forschungsschritt notiert oder verwertet werden konnten, festgehalten wurden (sh. Anlage 2: Forschungstagebuch).

\subsection{Vorbereitung}

Wie bereits in Kapitel 1.2 dargestellt, greift diese Arbeit zurück auf die Daten der Kasseler Coaching-Studie. Informationen zur Kasseler Coaching-Studie und ihrem Zweck können dem vom Forschungsteam der Universität Kassel erstellten Projektflyer entnommen werden (sh. Anlage 3: Projektflyer zur Kasseler Coachingstudie).

Um Coachs und ihre Klientinnen für die Teilnahme an der Studie zu gewinnen, nutzte das Team der Kasseler Coaching-Studie die Vernetzung mit den Berufsverbänden Deutscher Bundesverband Coaching e.V. (DBVC), Deutsche Gesellschaft für Supervision und Coaching e.V. (DGSv) und Österreichische Vereinigung für Supervision und Coaching (ÖVS), um erfahrene Coachs und ihre Coachees durch Mailings und Hinweise in Publikationen anzusprechen. Als Nutzen für die Coachs wurde argumentiert:

- Rückmeldung zum Vorgehen und der Wirksamkeit als Coach im Vergleich zum Durchschnitt der Coachs, unter Wahrung der Anonymität der Coachees

- Bei Audioaufzeichnungen: individuelle telefonische Rückmeldung zur Coachee auf der Grundlage der Operationalisierten Psychodynamischen Diagnostik (OPD) nach der ersten Coaching-Sitzung

- Teilnahme an der Kasseler Coachingforschungs-Community, mit Workshops zu den Grundlagen psychodynamischer Diagnostik, und mit Informationen über Workshops, Projekte und relevante Veröffentlichungen zur Coachingforschung.

Trotz intensiver Bemühungen des Forscherinnenteams konnte über die Laufzeit der Studie von 2017 bis 2020 die ursprünglich angestrebte Stichprobengröße von N = 100 für die fragebogenbasierte Erhebung und von $n=30$ für die zusätzlich audiographierte Teilstichprobe 
nicht erreicht werden. Es zeigte sich, dass die angesprochenen Coachs große Zurückhaltung übten, ihre Coachees um die entsprechende Zeitinvestition für das Ausfüllen der Fragebogen zu bitten, und dass sie bei einer Audioaufzeichnung eine potenzielle Störung des vertraulichen Rahmens befürchteten. So erfolgte bis März 2020 insgesamt ein Rücklauf von 19 audiographierten Coachingprozessen, die die Grundgesamtheit für diese Studie bilden. Als Vorteil der Grundgesamtheit kann festgehalten werden, dass diese die Forderung von Wegener (2019, S. 37) nach ,hochwertiger“ Coachingforschung insofern erfüllt, als dass sie nicht nur, wie häufig zu beobachten, auf Coachingprozesse bei Studierenden zurückgreift, sondern auf professionell durchgeführte Coachings unter Beteiligung umfassend qualifizierter Coachs, bei denen anspruchsvolle Anliegen aus einem komplexen Arbeitskontext bearbeitet werden.

Aus der Grundgesamtheit der audiographierten Coachingprozesse wurde zunächst eine Übersicht erstellt, die die Kennnummer des Prozesses und eine Kurzinformation zum Coachingprozess einschließlich der Anzahl der Coachingsitzungen enthält. Dabei wurde durch Sichten der Fragebogendaten (sh. Anlage 4: Kasseler Coachingstudie, Fragebogendaten), soweit sie vorlagen, überprüft, inwieweit die Prozesse der für diese Arbeit zugrunde gelegten Definition von Coaching als dyadischer arbeitsweltbezogener Beratungsdienstleistung entsprachen. Dort, wo keine, unvollständige oder wenig aussagekräftige Fragebogendaten vorlagen, wurde die Überprüfung durch stichprobenartiges Hören der Audiographien vorgenommen. Dabei zeigte sich, dass zwei Prozesse keine arbeitsweltbezogenen Themen, sondern andere Aspekte wie Flugangst und Höhenangst bearbeiteten, so dass diese für die weitere Bearbeitung nicht mehr berücksichtigt wurden. Ein weiterer Prozess bezog sich zwar in Teilen auf arbeitsweltliche Situationen, überwiegend arbeitete die Klientin allerdings biographische Ereignisse und pathogene Beziehungsmuster auf, so dass dieser Prozess auch im Bereich der Psychotherapie angesiedelt sein könnte. Um nicht in eine Abgrenzungsproblematik zwischen Psychotherapie und Coaching zu geraten, wurde auch dieser Prozess bei der weiteren Bearbeitung nicht mehr berücksichtigt. Daraus ergab sich eine Gesamtstichprobe von 16 Coachingprozessen bzw. 79 Coachingsitzungen (sh. Anlage 5: Gesamtstichprobe - Übersicht; sh. Anlagen 6-21: Audiographien der Coachingprozesse). Die Coachingprozesse wurden von 7 unterschiedlichen Coachs (2 Frauen, 5 Männer) durchgeführt, die jeweils über mehrjährige Erfahrung als professionelle Coachs verfügen und das Coaching als externe, freiberuflich agierende Beraterinnen gegen Bezahlung durchgeführt hatten.

Für den weiteren Überblick wurden alle in der Gesamtstichprobe zur Verfügung stehenden Audiographien vollständig angehört, und in diesem Rahmen wurde ein erstes grobes Raster über die in den Gesprächen zu beobachtenden Experiencing-Stufen erstellt. Die Einschätzung zu den Stufen erfolgte auf der Grundlage des Forschungs- und Trainingsmanuals zur deutschen Fassung der Experiencing-Skala EXP (Dahlhoff \& Bommert, 1978), siehe Kapitel 4.6). Verschiedene Autoren (ebd., S. 88) empfehlen, das Rating zum Experiencing anhand von vier-Minuten-Stichproben aus den Gesprächen vorzunehmen und zum Vergleich unterschiedlicher Therapie- bzw. Coachingsitzungen jeweils die ersten vier Minuten aus der zweiten 
Hälfte eines Kontakts zu wählen. Die Autoren weisen auf den nicht linearen Verlauf der EXPWerte hin, so dass es wichtig ist, nach Möglichkeit die EXP-Werte aller wesentlichen Phasen des Prozesses zu erfassen. Für die Task-Analyse sequentieller Prozesse spielt der Verlauf der EXP-Werte über mehrere Coaching-Sitzungen hinweg keine Rolle, und gleichzeitig ist damit zu rechnen, dass es auch in der ersten Hälfte oder in der Schlussphase einer Coachingsitzung zu relevanten Experiencing-Erfahrungen kommen kann. Aus diesen Überlegungen heraus wurde der EXP-Wert (Modus-Wert) für jedes Gespräch jeweils im Abstand von 10 Minuten eingeschätzt. Für die Einschätzung wurde jeweils eine vier-Minuten Sequenz herangezogen. So wurden für den Wert nach 10 Minuten die Minuten 8 bis 12 berücksichtigt, für den Wert nach 20 Minuten die Minuten 18 bis 22 etc. Im Zweifelsfall, wenn beim ersten Hören die EXP-Stufe nicht eindeutig zu bestimmen war, wurde die höhere EXP-Stufe eingetragen. Vor der Durchführung des Rating wurde ergänzend zu den in Kapitel 4.6 vorgestellten Dimensionen der Skala eine Rating-Instruktion erstellt, die Stufenbeispiele und eine genaue Anleitung zum Rating enthält (sh. Anlage 1: Experiencing-Skala: Ratinginstruktionen und Stufenbeispiele).

Ergänzend wurden in diesem ersten Durchgang bereits Memos notiert über Aussagen, die für die spätere Auswertung hinsichtlich eventueller in der jeweiligen Sequenz auftauchender und im Zusammenhang mit Experiencing stehender psychologischer Aufgaben relevant erschienen. Mögliche Task-Marker, Non-Task-Marker oder andere besondere Auffälligkeiten wurden beim Schreiben der Memos zusätzlich mit grünem Textmarker hervorgehoben. Wenn an einer Stelle der Gespräche ein besonderer EXP-Spitzenwert erkennbar war, wurde auch dieser notiert. So entstand ergänzend zur Gesamtübersicht ein erstes Raster zu den einzelnen Prozessen (sh. Anlage 5: Die Coachingprozesse im Detail).

Ziel dieses Schrittes war neben der Erstellung einer Übersicht weniger die exakte Bestimmung des jeweiligen EXP-Wertes als vielmehr die Identifikation derjenigen Coachinggespräche, bei denen Experiencing eine Rolle spielen könnte und derjenigen Gespräche, bei denen Experiencing keine erkennbare Rolle spielt und die deshalb nicht in die weitere Auswertung einzubeziehen waren. Ein Beispiel hierfür sind solche Gesprächseinheiten, bei denen die kognitive Auseinandersetzung mit Fragen der Arbeitsgestaltung oder der Führung im Vordergrund stand und bei denen die Coach weniger als Reflexionshelferin und mehr als fachliche Inputgeberin in Anspruch genommen wurde.

\subsection{Eingrenzung der Stichprobe}

Nach der Erstellung des Überblicks über die Gesamtstichprobe erfolgte die Auswahl der Fälle, die bei der weiteren Untersuchung berücksichtigt werden sollten, in zwei Schritten.

Im ersten Schritt wurde zunächst die Gesamtstichprobe weiter eingegrenzt auf einzelne Coachingsitzungen, die möglicherweise Informationen zur Forschungsfrage enthalten könnten. Im zweiten Schritt erfolgte dann das Sampling im engeren Sinn, also die Identifikation 
derjenigen Gesprächsepisoden, die im Rahmen der Task Analyse näher untersucht werden sollten.

Für den ersten Schritt, die Eingrenzung der Stichprobe, wurde festgelegt, dass von den Coachingprozessen insgesamt nur solche Gesprächseinheiten für die Forschungsfrage relevant sind, die entweder einen möglichen Hinweis auf einen Task Marker beinhalten oder bei denen ein Felt Sense bzw. ein Experiencing-Prozess beobachtbar ist. Als Beobachtungsinstrument für das Experiencing diente die Experiencing-Skala (EXP-Skala) (Dahlhoff \& Bommert, 1978), deren Stufen 4 bis 7 einen Experiencing-Prozess im Sinn einer Wahrnehmung des unmittelbaren Fühlens und Erlebens von einem innen liegenden Standpunkt heraus abbilden. Dem entsprechend wurden die dem Auswahlkriterium EXP Stufe 4 oder höher entsprechenden Coachingsitzungen in die Auswahl aufgenommen. Allein ein Spitzenwert von EXP Stufe 4 bei einer ansonsten durchgehend niedrigeren EXP-Stufe wurde dabei nicht als ausreichend aussagekräftig eingeschätzt, so dass für die Auswahl der Modus-Wert berücksichtigt wurde. Da die Kriterien für den Task Marker zu diesem Zeitpunkt noch nicht festgelegt waren, wurden in diesem ersten Schritt keine weiteren Auswahlkriterien zugrunde gelegt, und es ergab sich eine Stichprobengröße von N = 31 Coachingsitzungen. Um diese Auswahl von der Gesamtstichprobe abzugrenzen, wird sie im weiteren Verlauf als „,relevante Stichprobe“ bezeichnet (sh. Anlage 22: Relevante Stichprobe).

Aufgrund des großen Material- und Datenumfangs der relevanten Stichprobe wurde aus Gründen der Ökonomie von einer vollumfänglichen Transkription aller zur relevanten Stichprobe zählenden Coachingsitzungen abgesehen und entschieden, diese Auswahl anhand der Audiographien zu treffen und die jeweiligen Coachinggespräche Zug um Zug im Rahmen der weiteren Untersuchungsschritte zu transkribieren.

\subsection{Transkription}

Nach Gläser \& Laudel (1999, S. 5) dienen Transkripte als Hilfsmittel, um zu Aussagen über das eigentliche Untersuchungsobjekt zu gelangen. So wurden zur genaueren Analyse der zu untersuchenden Gesprächseinheiten Transkripte erstellt, anhand derer dann die Task Analyse erfolgen konnte. Als Orientierung für die Transkription dienten die Regeln von Kuckartz, Dresing, Rädiker \& Stefer (2007, S. 27ff) und von Dresing \& Pehl (2018) zur inhaltlichsemantischen Transkription, die sich auch im Transkriptions-Leitfaden zur Kasseler Coachingstudie (sh. Anlage 23: Leitfaden zur Erstellung von Transkripten) wiederfinden. Die Transkripte wurden vollständig anonymisiert, zur Nachvollziehbarkeit der Anonymisierung wurden Legenden gebildet, die separat dokumentiert wurden (sh. Anlagen 24-32: Legenden zu den einzelnen Prozessen).

Pausen und Besonderheiten in der Transkription wurden analog zum vorliegenden Transkriptions-Leitfaden gekennzeichnet. Wo es bei den Markierungen geringe Abweichungen zu den Vorgaben des Leitfadens gab, wurden dies ergänzend in den Legenden vermerkt. Individuelle 
Unterschiede im Umgang mit den Vorgaben des Leitfadens kamen auch zustande, weil die Transkriptionen teilweise von Studierenden oder Mitarbeitenden der Universität Kassel, und teilweise vom Autor dieser Studie bzw. einer Mitarbeiterin des Autors erstellt wurden. Da diese Unterschiede sich aber lediglich auf die äußere Form wie z.B. die Markierung von Anonymisierungen bezogen, konnte davon ausgegangen werden, dass sie keinen Einfluss auf die Task Analyse haben würden.

Die jeweiligen Transkript-Dokumente wurden analog zur Kennung des Coachingprozesses benannt und in der Übersicht zur relevanten Stichprobe verlinkt (sh. Anlagen 33-52: Transkripte zu den einzelnen Coachingsitzungen; sh. Anlage 6: Relevante Stichprobe).

\subsection{Aufgabe: Umgehen mit dem Unklaren}

Im Zentrum dieser Arbeit steht die Frage nach intraindividueller Veränderung im Kontext experienzieller Prozesse. Ziel der Veränderung ist eine Erkenntnis oder eine affektive Umgestaltung, beispielsweise hinsichtlich der eigenen Einstellungen oder Motivation, die zur Erarbeitung einer neuen oder veränderten Handlungsstrategie führt. Nach dem experienziellen Veränderungskonzept geht intraindividuelle Veränderung und das Lösen von Problemen im Allgemeinen mit tief im Inneren eines Menschen gefühlten emotionalen Ereignissen einher (Gendlin, 1978, S. 8). Diese Ereignisse werden dabei nicht nur als Begleiterscheinung, sondern in gewisser Weise als grundlegend für die Lösung erachtet.

Experiencing wird dann als besonders hilfreich erachtet, wenn bei der Bearbeitung eines Themas der Eindruck entsteht, dass da noch mehr sei, als im Augenblick erfasst werden kann, oder dass es um Aspekte geht, die momentan sprachlich nur begrenzt formuliert werden können. Damit lässt sich Experiencing nicht nur als Prozess verstehen, sondern gleichzeitig als psychologische Aufgabe: Neben dem Thema, das bearbeitet werden soll, richtet sich die Aufmerksamkeit der Klientin auf das aktuelle Erleben, auf das Unklare, Implizite, und darauf, wie dieses Erleben zur Problemlösung genutzt werden kann. Das Unklare kann sich dabei zunächst als Problem darstellen, das die Klientin bei ihrem Lösungsprozess behindert, gleichzeitig kann es im weiteren Prozess auch als Ressource zur Lösung genutzt werden.

Hinweise, um welche Situationen es dabei gehen kann, können aus den von Greif (2008, sh. Kapitel 2.2.2) beschriebenen Wirkfaktoren im Coaching abgeleitet werden. Unklar können Situationen sein, wenn

- intensive Gefühlsregungen auftauchen, die von der Coachee zunächst nicht eingeordnet werden können, wenn

- Problemsituationen sich einem tieferen Verständnis verschließen und Ziele sich nicht zufriedenstellend klären lassen, oder wenn

- zunächst keine internen oder externen Ressourcen gefunden werden können, die der Klientin für die Lösung des Problems zur Verfügung stehen, oder wenn

- die Klientin sich zwar intensiv mit einer Situation oder mit sich selbst beschäftigt, 
daraus aber kein auf eine Lösung ausgerichteter Prozess entsteht.

Es ist nicht davon auszugehen, dass das Unklare dabei immer als solches benannt werden kann, oder dass die Klientin einen expliziten Klärungsauftrag im Coaching formuliert. Vielmehr ist zu vermuten, dass das Unklare sich im Coachinggespräch eher diskret artikuliert in Form von undeutlichen, zögerlichen, stockenden, vielleicht auch in sich widersprüchlichen Aussagen.

So soll die in dieser Studie zu untersuchende Aufgabe umschrieben werden als „Umgehen mit dem Unklaren“ im Sinn einer Selbstklärung. Mit dem Unklaren ist dabei gemeint, dass die Klientin spürt, dass da noch etwas nicht Spezifizierbares ist, was sie aktuell auch auf einer affektiven Ebene berührt oder irritiert und zunächst noch nicht zu einer Lösung durchdringen lässt. Dazu kann auch gehören, dass die Klientin verwundert ist über sich selbst oder ihre eigene Reaktion in einer bestimmten Situation. Die Aufgabe der Klientin ist in diesen Fällen, bei dem Unklaren zu verweilen, bisher implizit vorhandene Aspekte darin zu erkennen und diese Aspekte zur Lösungsfindung zu nutzen.

Entsprechend dieser Vorüberlegungen wurden aus der relevanten Stichprobe $(\mathrm{N}=31) \mathrm{zu}-$ nächst drei Gesprächssequenzen ausgewählt, die aus der Sicht des Forschers idealtypisch für die beschriebene Task sind. Diese Sequenzen wurden auf solche Klientinnenäußerungen reduziert, die die Task möglichst deutlich kennzeichnen. Aussagen, die sich nicht direkt auf die Task beziehen und Aussagen der Coach wurden nicht berücksichtigt, und entsprechende Stellen wurden mit doppelten Auslassungspunkten (.....) gekennzeichnet. Folgende Sequenzen kamen dabei zur Auswahl:

Task Marker - Beispiel 1:

„Dieses "Du denkst nur an Dich". Ja? Also die haben letztendlich genau die Argumente rausgeholt, und haben mich so damit treffen können. Ich war wirklich zum großen Teil also, ja fast handlungsunfähig. Ich konnte darauf kaum reagieren. Und als ich das jetzt gelesen hab, da hab ich "Oh das gibt's doch gar nicht. Das ist ja so komplett absurd. Das ist so völlig schräg, was die mir vorgeworfen haben". Da hätte ich aufplatzen können vor Wut, ja? ...... Aber ich merk gerade, wie das in mir pocht, ja? ...... Dass ich immer noch unglaublich wütend bin. Und aber auch, auch eigentlich fassungslos. Also ja, dass ich merk "Boah, ich hab mir das alles bieten lassen. Hallo! Geht's noch?"““ (Klientinnenaussage, Coachingsitzung 2017_1805_01\#1, Zeitstempel \#00:56:09-2\# bis \#00:57:31-5\#)

Begründung des Task Markers: Die Klientin spricht über eine Situation aus der Vergangenheit, mit der sie sich immer noch auseinandersetzen muss, die sie wütend macht. Das Unklare zeigt sich darin, dass eine intensive Gefühlsregung auftaucht, die sie auch körperlich spürt und zunächst nicht einordnen kann. Dabei verweilt sie zunächst für einen Moment. Das Entsetztsein darüber, dass sie sich so viel hat bieten lassen, kann als Hinweis gedeutet werden, dass sie daran interessiert ist, künftig anders mit vergleichbaren Situationen umzugehen (Veränderungswunsch), aber bisher noch keinen Lösungsansatz gefunden hat. 
Task Marker - Beispiel 2:

„Obwohl es etwas ist, was mir sehr bewusst ist, dass ich das machen muss, bald, ja, so ein Gespräch führen. Weil ich will, dass dieser Bereich bald funktioniert. ...... Ich kann das, zum Beispiel, es gibt so gewisse Menschen, wo mir das wahnsinnig schwerfällt, ja? ...... Ahm (...), das sind zum Beispiel Menschen, von denen ich (...) ich komme, ich komme mir so quasi emotional total unter Druck gesetzt vor von ihnen. Weil ich weiß, also da steht ein Schicksal dahinter, und, (...) ...... Und es gibt da für mich so irgendwie so, so eine unsichtbare, oder, ich weiß auch nicht wie man sagen soll, so Grenze. Wenn man denkt, ich weiß nicht, die bringen so quasi, irgendein Schutz oder, ich kann es nicht, ich kann es nicht wirklich ausdrücken. ..... Ja, so (...) oder ich weiß nicht, das zurückgesetzt, vielleicht können wir da mal irgendwie das anders formulieren, ahm, ja, im Prinzip, es geht schon um das, ich weiß nicht, das zurückgesetzt, das Wort ist vielleicht nicht so ganz." (Klientinnenaussage, Coachingsitzung 2016_1119a_01\#1, Zeitstempel \#0:34:45-3\# bis \#0:41:11-9\#)

Begründung des Task Markers: Die Klientin beschreibt eine unklare Problemsituation, bei der sie ihr eigenes Agieren, bzw. ihr Nicht-Agieren gerne tiefer verstehen würde. Sie spricht zögerlich, unterbricht sich selbst oft, macht Pausen, sucht nach passenden Worten, verweilt damit zunächst bei dem Unklaren, ohne es letztlich ausdrücken zu können. Dabei kommt sie zunehmend auf ihre Gefühle und ihre Motivation zu sprechen. Der Veränderungsimpuls besteht insofern, als dass sie weiß, dass ihre berufliche Rolle von ihr ein anderes Agieren als bisher erfordert und sie sich diesem Erfordernis stellen möchte.

Task Marker - Beispiel 3:

„Wenn ich mir die Fragen versuche zu beantworten, warum hadere ich denn überhaupt, ne, mit dem, mit dem Bleiben. ...... Ja. Dann ist das nicht so die inhaltliche Geschichte, sondern es ist, das habe ich auch im ersten Gespräch schon gesagt zu Anfang, es ist diese persönliche Geschichte. Ich habe so eine Unsicherheit, ob da, ähm, in Bezug auf das Vertrauen. Ne, also gibt es da ...... Also, was würde da der nächste Schritt sein. Also, wenn ich mich jetzt auf einen Kompromiss einlasse, haben wir dann eine Basis, auf der wir langfristig gut zusammenarbeiten können, oder ist das der erste Schritt, äh, um sozusagen den nächsten Schritt zu gehen, der dann aber einfach vielleicht kostenmäßig nicht so zu Buche schlägt, um mich noch eine Weile zu haben. ...... Und wenn das nicht wäre, ne, das habe ich mir auch versucht zu beantworten. Wenn das nicht wäre, diese Unsicherheit in Bezug auf das Vertrauen, dann, ahm, hätte ich auch dann, dann hätte ich gar keine Bedenken, da bleiben zu wollen, weil da, wie gesagt, so viele Dinge, inhaltlich, auch von den, und auch von den Rahmenbedingungen dafür sprechen.“ (Klientinnenaussage, Coachingsitzung 2017_0118_01\#2, Zeitstempel \#0:11:08-2\# bis \#0:12:41-4\#)

Begründung des Task Markers: Die Klientin äußert Unbehagen und Unsicherheit als Ursache ihres Haderns mit dem Bleiben. Sie geht nicht schnell darüber hinweg, sondern betont die 
Relevanz ihrer Unsicherheit. Sie reflektiert die Situation, kommt aber auch im Rahmen der Reflexion nicht zu einer Lösung. Das Unsicherheitsgefühl bleibt für sie spürbar und verbunden mit der Unklarheit, auf welche internen oder externen Ressourcen sie zur Lösung zurückgreifen könnte. Ein Veränderungswunsch wird nicht expliziert, besteht aber indirekt, weil sie eine Antwort auf das Hadern mit dem Bleiben sucht. Im weiteren Verlauf wird aus der Aufgabenumgebung dieses Task Markers zwar erkennbar, dass in diesem Fall kein vorwärtstragender Prozess im Sinne des Experiencing entsteht. Trotzdem wird hier zunächst die Aufgabe „Umgehen mit dem Unklaren“ markiert, auch wenn die Aufgabenerledigung dann möglicherweise keinen idealtypischen Verlauf nimmt.

Abgegrenzt werden kann die Aufgabe von Elementen, die eher den Charakter einer Fachberatung oder eines Fachdiskurses haben. In den Coachinggesprächen tauchen immer wieder Episoden auf, innerhalb derer die Klientin die Coach als Sparringspartnerin für fachliche Diskurse, z.B. über Führungsmodelle oder Organisationsmodelle, anfragt. Wenn im Diskurs dann Unklares thematisiert wird, dabei aber überwiegend kognitive Überlegungen im Vordergrund stehen und eine Beteiligung affektiver Ebenen nicht erkennbar ist, würde diese Task eher als „fachliche Selbstvergewisserung“ bezeichnet werden und damit nicht in die Kategorie „Umgehen mit dem Unklaren“ im Sinn einer Selbstklärung fallen.

Auch wenn beim Experiencing das Körpergefühl eine Rolle spielt, ist ein benanntes Körpergefühl nicht per se schon ein Hinweis, dass es hier um den erlebensorientierten Umgang mit Unklarem geht, so dass auch dahingehend eine Abgrenzung vorzunehmen ist:

Non-Task-Marker - Beispiel 1:

„Körpergefühl (unv.). Vielleicht mehr Ruhe einfach. ..... Ich bin ruhiger.“ (Klientenaussage, Coachingsitzung 2015_0111_01\#2a, Zeitstempel \#00:08:06-4\#)

Kein Task Marker: Im Vorfeld dieser Klientenaussage fragt der Coach nach einem Körpergefühl in einer bestimmten Situation. Der Klient beschreibt dieses dann wie aus einer externen Beobachtungsposition heraus. Er benennt zwar ein gewisses Körpergefühl, aber ohne erkennbare innere Beteiligung, und das Gefühl wird dann auch nicht zum Ausgangspunkt einer weiteren Exploration. Es ist keine Unklarheit zu erkennen, an der der Klient arbeitet, und das Erleben wird nicht weiterbearbeitet.

Non-Task-Marker - Beispiel 2:

„Also letztendlich müsste ich jetzt vor den Ferien nochmal ein Gespräch mit ihr führen, weil sich die Situation auch noch zuspitzt und ihr Sohn jetzt im Sommer auch noch in die Schule kommt (lacht) und ich mich frage, wie wird das dann?“" (Klientinnenaussage, Coachingsitzung 2017_1805_01\#3, Zeitstempel \#01:11:16-9\#)

Kein Task Marker: Die Klientin arbeitet an einem Problem in der Kommunikation mit einer anderen Person und erkennt während des Bearbeitungsprozesses die Notwendigkeit zu einem Handlungsschritt (Gespräch führen). Es ergibt sich dabei aber keine weitere Unklarheit für sie, sondern sie erarbeitet auf der Grundlage einer sachlichen Situationsanalyse die Notwendigkeit eines Handlungsschritts, und es ist weder an den Inhalten der verbalen Äußerungen 
noch an der Art und Weise des Sprechens erkennbar, dass die Klientin im Blick auf das Thema noch etwas Implizites erlebt, was expliziert werden sollte.

Non-Task-Marker - Beispiel 3:

„Es hat einen Einfluss auf die Organisationsstruktur: Es hat einen Einfluss darauf, also diesen nicht-informationellen Einfluss auf das „Wie stelle ich mich gegenüber meiner Kollegin“. Hat einen Einfluss auf dieses ganze Leitungsteam. Hat einen Einfluss auf das ganze Heim. Operativ betrachtet macht das gerade keinen Unterschied, weil, ich betone das nochmal, ich habe da Narrenfreiheit gerade. Das ist sehr (...). Es ist unangenehm, tatsächlich unangenehm. Unnatürlich. Weil selber fehlt einem die Leitung. Da fragt man sich natürlich, da kann ich auch gleich selber alles machen. ..... Ja. Also ich denke es wird auch zukünftig nicht so sein, dass es eine Geschäftsführung für beide Häuser gibt. Es wird immer noch eine Verantwortungsebene geben müssen, die vor Ort die Hauptverantwortung trägt.“ (Klientenaussage, Coachingsitzung 2017_2119_01\#1, Zeitstempel \#00:05:56-0\# bis \#00:06:12-2\#)

Kein Task Marker: Es liegt zwar ein Bezug zum eigenen Erleben vor („Es ist unangenehm“), aus dem ein Veränderungswunsch abgeleitet werden könnte. Allerdings liegt keine Indikation im Sinn einer Unklarheit vor, an der gearbeitet werden sollte. Es könnte allenfalls ein Fachdiskurs entstehen, bei dem die Organisationsstruktur weiter thematisiert wird.

Bei der weiteren Durchsicht des Materials wurde nach ergänzenden Beispielen gesucht, anhand derer ergänzende Einsichten zur Spezifizierung des Task Markers gewonnen werden könnten. So konnten zwei weitere Beispiele herangezogen werden:

Task Marker - weiteres Beispiel 1:

„Es ist tatsächlich nicht so, dass das einen Riesendruck auf mich aufbaut und, dass ich mich da sehr, sehr unwohl fühle in der Situation. Ich akzeptiere die Situation, wie sie ist, weil ich weiß, dass sie so ist. Meine Kollegin schätzt die ganze Lage etwas anders ein, nämlich die hat tatsächlich dieses idealistische Bild, was sie jetzt glaube ich einfach langsam begraben kann. Aber ich weiß nicht, irgendetwas an der Gesamtlage stört mich so massiv, dass ich nicht weiß, wo das eigentlich hinführt. Unterm Strich. Jetzt erstmal Tatsache ist dieses Gespräch morgen für mich, in dem ich sehr klar sein möchte und welches für mich auch zielführend sein soll beziehungsweise wegweisend...... Auch da bin ich von mir selber in dem Moment einfach ein bisschen irritiert.“ (Klientenaussage, Coachingsitzung 2017_2119_01\#1, Zeitstempel \#00:12:43-2\#)

Task Marker: Der Klient gibt einerseits an, nicht belastet zu sein. Gleichzeitig gibt er an, sich in der benannten Situation unwohl zu fühlen. Er ist von sich selbst irritiert, kann nicht einordnen, woher diese ,innere Störung“ kommt. Zwar sucht er die Antwort im weiteren Verlauf im Verhalten seiner Vorgesetzten, aber die Hinweise „,nicht belastet“ und gleichzeitig „unwohl“" weisen auf eine implizite affektive Komponente hin, die mit einer Unklarheit im Sinn 
des Nicht-Einordnen-Könnens und eines widersprüchlichen Erlebens der eigenen Gefühlsregungen einhergeht. Der Wunsch, mehr Klarheit zu erlangen, wird anhand des Ausblicks auf ein bevorstehendes Gespräch deutlich.

Task Marker - weiteres Beispiel 2:

„Ich bestimme wie es mir geht. ..... Ahm, ja, was heißt, wer anders? Es sind dann eher Gefühle oder Gedanken oder Zustände (okay), die, die mich, äh, die, die ich jetzt in dem Moment auch nicht unbedingt brauche. ...... Es gibt so, so was weiß ich, ob das, ahm, (...). Ja, genau. So stressige Momente sind oder leichte Anflüge von Angstzuständen, also jetzt nicht, aber doch, irgendwie schon. ...... Und die Angst ist dann kurz, ist dann da, und dann fühle ich mich unwohl, da habe ich immer das Gefühl, das ist jetzt irgendwie alles fremd. Das bin ja nicht ich. Das kommt von irgendwoher. Das ist jetzt nicht eine Sache, die, ah / ..... Das sind Versagensängste. Oder auch Ängste, dass, äh, (unv.) Versagen. In letzter Zeit ich weiß auch nicht, da hatte ich so gedacht, ob das jetzt mit dem, ich werde jetzt nächstes Jahr vierzig. Geht das mit dem Metabolismus los? Körper stellt sich um? Kriege ich jetzt den Bauch, den mein Vater hat? Geht das jetzt los, dass ich / deswegen, also ich habe das Gefühl, ich muss jetzt tatsächlich auch, aufs Coaching bezogen, also überhaupt auf diese, nicht nur Coach, auch Fitnessstudio, egal was, ich MUSS jetzt was machen." (Klientenaussage, Coachingsitzung 2015_0111_02\#1, Zeitstempel \#00:27:03-4\# bis \#00:29:14-1\#)

Task Marker: „Ich bestimme wie es mir geht“ ist vom Klienten im ersten Teil der Coachingsitzung als Ziel für das Coaching benannt worden, zunächst eher unspezifisch im Sinn einer gewissen Selbststeuerung. Hier greift er das Thema auf, nachdem der Coach zuvor gefragt hatte, woran sie heute arbeiten sollten. Die Episode wird damit direkt mit einem Veränderungswunsch bzw. Task Marker eröffnet, auch wenn dieser noch nicht weiter konkretisiert ist. Auffällig ist, dass der Klient, der ansonsten elaboriert und flüssig spricht, nach passenden Formulierungen sucht, viele Pausen macht, nicht weiß, wie er in Worte fassen soll, was ihn beschäftigt. Er kann seine Versagensängste zwar beschreiben, aber nicht einordnen. Und er weiß noch nicht, ob die Antwort auf sein Thema tatsächlich im Coaching zu finden ist, oder ob er anderweitig, z.B. im Sport, danach suchen sollte. Gleichzeitig erlebt der Klient einen starken inneren Drang, etwas - was auch immer - zu tun, um das unklare Problem zu lösen. Zusammenfassend lassen sich aus diesen Beispielen folgende Kernmerkmale des Task Markers „Umgehen mit dem Unklaren“ herausarbeiten:

- Die Klientin beschreibt eine Unklarheit, eine Unsicherheit oder Irritation, die sie in sich selbst entdeckt und die ihre Gefühle oder Fragen der Haltung oder Motivation tangiert

- Mit dem Unklaren, dem Innehalten oder der Irritation geht ein Klärungsanliegen einher, bei dem die Klientin eine gewisse Problematik beschreibt, die sie verändert 
wissen möchte. Der Veränderungswunsch muss dabei allerdings nicht direkt formuliert sein, als Merkmal für den Task Marker genügt es, wenn er sich indirekt aus den Äußerungen der Klientin ableiten lässt

- Oft entsteht über die verbalen Äußerungen hinaus auch ein Moment des Innehaltens bzw. Zögerns. Die Klientin verlangsamt oder verlagert den Gesprächsprozess, weil ihr etwas bei sich selbst auffällt, was sie nicht ganz einordnen kann. Es ergeben sich Pausen im Gespräch, oder zögerliche oder unvollendete Formulierungen, die erkennen lassen, dass es der Klientin schwerfällt, das in Worte zu fassen, was in ihrem Inneren vorgeht.

Die derart gekennzeichnete Aufgabe des Umgehens mit dem Unklaren kann bei erfolgreicher Bewältigung eine Bedeutung für das Gelingen des Coachingprozesses insgesamt haben, und es kann davon ausgegangen werden, dass die erfolgreiche Bewältigung zu einem signifikanten Veränderungsmoment bei der Klientin führt.

Nicht zur Aufgabe „Umgehen mit dem Unklaren“ gehören Aspekte wie:

- die Beschreibung eines Körpergefühls aus der Vergangenheit, das nicht aktuell erlebt oder weiterverarbeitet wird

- Äußerungen zum eigenen Erleben, die nicht im Kontext einer Problemindikation stehen und bei denen die Klientin keinen Hinweis gibt, dass sie daran arbeiten möchte

- Sachfragen, deren Erörterung nicht erkennen lässt, dass die Klientin sich mit etwas Unklarem bei sich selbst beschäftigt, das sie momentan nicht einordnen kann.

Die beschriebenen Merkmale der Aufgabe zeigen sich bei der ex post Betrachtung des Materials, die Festlegung des Task Markers erfolgt also nicht aus der Coach- oder Klientinnenperspektive, sondern aus der Perspektive der Forschenden, die versucht, das Erleben der Klientin nachzuvollziehen. Ziel der Aufgabenbearbeitung ist eine In-Session-Veränderung, also ein Prozess, der sich innerhalb einer bestimmten Gesprächssequenz vollzieht und der auch als Veränderung erkennbar ist, zum Beispiel in Form eines Aha-Erlebnisses, eines Felt Shift oder einer sonstigen Klärung.

\subsection{Kognitive Karte: bottom up}

Die kognitive Landkarte des Autors, an der er sich bei der weiteren Ausarbeitung orientiert, ergibt sich in weiten Teilen aus den Kapiteln zum Coaching (Kapitel 2) und zum Experiencing einschließlich des Diskurses zum Experiencing (Kapitel 3 und Kapitel 4). Wie in Kapitel 2 dargelegt, geht es im Coaching darum, dass sich „Neues“ ereignen soll, das der Klientin alternative Handlungsmöglichkeiten oder Sichtweisen eröffnet. Der Schwerpunkt der im Coaching selbst stattfindenden Veränderung liegt dabei im Wesentlichen im Bereich der intrapersonalen Veränderung, also auf der Lernebene hinsichtlich der Einsichten und Einstellungen, des Wissens oder der Motivation. 
Aufbauend auf das Menschenbild der Humanistischen Psychologie geht der Autor davon aus, dass Veränderung nicht nur „top-down“ im Sinn rationalen Explorierens von Situationen von statten geht, sondern zum großen Teil auch „,bottom-up“, also durch die Auseinandersetzung mit dem eigenen emotionalen Erleben und den daraus entstehenden schrittweisen Verhaltensoder Konzeptänderungen. Impulse zur Veränderung müssen damit nicht primär von außen gesetzt werden, sondern entwachsen der Neigung des Menschen, sich konstruktiv in Richtung Selbstverwirklichung und Unabhängigkeit zu entwickeln.

Im Zentrum dieser Studie stehen Fragen nach dem Experiencing der Klientin und nach dem Zusammenwirken von Experiencing und Veränderung. Der Fokus liegt dabei auf dem klientinnenseitigen Geschehen und besonders der Phase, in der ein Bewusstsein für Veränderung entsteht, er liegt weniger auf der Interaktion oder dem Verhalten der Coach, das zwar sicherlich relevant, aber für die Exploration der intrapersonalen Veränderung nicht von vordergründiger Bedeutung ist. Wenn von Experiencing die Rede ist, kann dies nach dem Verständnis des Autors sowohl eine körperlich gefühlte Komponente im Sinn des Felt Sense einschließen als auch ein im Augenblick stattfindendes emotionales Erleben mit einer gefühlten, unmittelbaren, nicht immer vollständig explizierbaren persönlichen Bedeutung.

Weiterhin geht der Autor davon aus, dass das Ergebnis der In-Session-Veränderung im Coaching mit einer gefühlten Erleichterung bei der Klientin einhergeht. Dazu finden sich Analogien zwischen Gendlins Felt-Shift-Konzept und den Ansätzen der Critical-Moments (de Haan \& Nieß, 2012, 2018), der Bedeutsamen Momente (Wegener, 2019) oder der Aha-Erlebnisse (Greif \& Riemenschneider-Greif, 2018) (sh. Kapitel 2.3). Diese Erleichterung, wie überhaupt die innerpsychischen Prozesse der Veränderung, bilden sich in der Regel in verbalen Äußerungen oder direkt beobachtbaren Handlungen der Klientinnen ab und werden dadurch erkennbar und beschreibbar.

\subsection{Kontext: Experiencing}

Der Umgang mit Unklarem kann im Coaching auf verschiedene Arten erfolgen. Vor dem Hintergrund der Fragestellung dieser Arbeit und der Forschungslandkarte des Autors sollen hier solche Aufgabenerledigungen untersucht werden, bei denen sich ein Experiencing beobachten lässt, so dass das aktuelle Selbsterleben Teil eines vorwärts tragenden Prozesses wird. Aus der Therapieforschung ist bekannt, dass ein höheres Experiencingniveau bei der Bearbeitung problematischer Kernpunkte regelmäßig einhergeht mit einem signifikant positiven Ergebnis (vgl. Kapitel 3.4; Watson et al, 2010). Erkennbar ist das Experiencingniveau am Ausmaß des Selbsterlebens, wie es mittels der Experiencing-Skala erfasst werden kann. Das Selbsterleben ist zu unterscheiden von der Selbstreflexion, die im Coaching ebenfalls eine zentrale Rolle einnimmt (vgl. Kapitel 2), hier aber als Kontext für die Untersuchung nicht als ausreichend erachtet werden würde, solange nicht gleichzeitig ein Selbsterleben in der aktuellen Situation beobachtet werden kann. 
Als Mindestmaß der prozesshaften Bezugnahme auf das Selbsterleben kann ein Übergang von Experiencing Stufe 3 (EXP 3) zu einer höheren Stufe gelten. An dieser Schwelle verändert sich der Standpunkt der Sprechenden, der sich bisher quasi außerhalb des eigenen unmittelbaren Erlebens befand, hin zu einem inneren, im unmittelbaren Fühlen und Erleben liegenden Standpunkt (Dahlhoff \& Bommert, 1978, S. 75; Watson et al, 2010, S. 150). Ab Stufe 5 der EXP-Skala (EXP 5) verarbeitet die Klientin ihre inneren Erlebnisse weiter, so dass anzunehmen ist, dass das Experiencing spätestens ab dieser Stufe mit einem Veränderungsprozess einhergeht. Insgesamt kann davon ausgegangen werden, dass Experiencing dann im Prozess wirksam wird, wenn nicht nur ein kurzzeitiger Spitzenwert der Stufe 4 oder höher erreicht wird, sondern wenn es gelingt, dass die Klientin für eine gewisse Sequenz bei ihrem Selbsterleben verweilen kann.

Als gelöst kann die Aufgabe betrachtet werden, wenn ein Aha-Effekt oder ein Felt Shift eintritt, die Klientin also auf irgendeine Art von einer emotionalen Erleichterung oder einer neu gewonnenen Erkenntnis berichtet, die ihre Selbststeuerung verbessert und sie darin bestärket, sich in ihrem Umfeld als souveräne Gestalterin zu bewegen.

Hinweisgeber auf die Lösung können neben einem direkt geäußerten Felt Shift auch die Merkmale signifikanter Veränderungsmomente sein, wie De Haan \& Nieß (2012, S. 202) sie beschreiben:

- Momente des Lernens, bei denen eine neue Einsicht entsteht, oder neue Verbindungen oder Perspektiven aufgegriffen werden

- positive oder negative Veränderungen in Beziehungen

- direkte Handlungen seitens der Klientin, die in diesem Moment eine hohe Bedeutung hat

- besonders intensive Emotionen auf Seiten der Klientin

- aufkommende Zweifel, die eine hohe Bedeutung haben.

So entstehen besondere Momente, in denen sich für die Klientin etwas zum Positiven wendet und bei denen sie Veränderungsprozesse im Sinn der Ausbildung funktionaler Deutungs- oder Handlungsmuster durchläuft. Als direkte Beobachtungsmerkmale für den Felt Shift können herangezogen werden:

- neue Gefühle, die in dem Moment Erleichterung verschaffen

- produktive, konstruktive, die Autonomie fördernde Bedeutungen, die im Zusammenhang mit dem Selbsterleben geäußert werden

- $\quad$ eine positive Selbstbewertung

- ein Handlungsplan, der mit einer neu gewonnenen Zuversicht einhergeht.

In der weiteren Erarbeitung werden daher solche Prozesse der Aufgabenerledigung als gelungen definiert, die im Kontext von Experiencing, messbar an EXP Stufe 4 oder höher (ModusWert EXP $\geq 4$ ), erfolgen, und bei denen ein Felt Shift bzw. ein anderweitiger signifikanter Veränderungsmoment im Sinn der Ausbildung alternativer funktionaler Deutungs- und Handlungsmuster zu beobachten ist. 
Wie bereits erwähnt, wird ein spezifisch focusingorientiertes oder personzentriertes Vorgehen der Coach nicht als Rahmenbedingung für die Untersuchung vorausgesetzt.

\subsection{Theoretisches Modell: vom Unklaren zum Felt Shift}

Dem in dieser Arbeit zu untersuchenden bzw. mit den empirischen Befunden abzugleichenden theoretischen Modell liegen neben den aktuellen Erkenntnissen zu Coachingprozessen (sh. Kapitel 2) in erster Linie experienzielle Konzepte zugrunde. In Anlehnung an Rice \& Saperia (1984, S. 31) und das in Kapitel 3 vorgestellte Konzept des Experiencing sollen folgende Vorannahmen gelten:

- Die Aktualisierungstendenz als die von Natur aus dem Menschen innewohnende Neigung, sich konstruktiv in Richtung Wachstum, Selbstverwirklichung und Unabhängigkeit zu entwickeln, ist ein wesentlicher und im Coaching zu unterstützender Faktor beim Lösen von Problemen und beim Gestalten von Veränderung

- Wenn die entsprechenden Bedingungen gegeben sind, können Klientinnen ihre inneren Erfahrungen wahrnehmen und diese zur Weiterentwicklung nutzen. Eine Deutung oder Bewertung der inneren Erfahrungen durch Dritte ist hierzu nicht notwendig und in der Regel kontraindiziert

- Menschliches Verhalten entwickelt sich auf der Grundlage der jeweiligen Wahrnehmungen und Wirklichkeitskonstruktionen. Ändert sich die Wahrnehmung, verändern sich auch die innere Reaktion und das äußere Verhalten

- Das bewusste Wahrnehmen und Gewahrwerden des inneren Erlebens führt zu einer Reorganisation des Selbstkonzepts, die einher geht mit einer höheren Kongruenz und Stimmigkeit. Experiencing umfasst dabei alle durch Sprache und Bilder symbolisierbaren, also bewusstseinsfähigen Prozesse, die im Individuum vor sich gehen.

Ein wesentliches Element dieses „bottom-up“-orientierten bzw. von innen (inneres Erleben) nach außen (äußere Handlungen) gerichteten Veränderungskonzepts ist die Aufmerksamkeitsfokussierung auf das Selbsterleben (,,referring inward“), und in einem weiteren Schritt die Bezugnahme auf dieses Selbsterleben (,felt referent“). Durch das Verweilen und Bezugnehmen auf das innere Erleben entsteht ein Felt Sense als innerer Bezugspunkt für den weiteren Prozess. Generelles Ziel des Prozesses bzw. der Veränderung ist die Verbesserung der Selbststeuerung und die Entwicklung von Deutungs- und Handlungsalternativen, die das Individuum in seiner Souveränität bestärken.

Im Coachingprozess kann die aktuell von der Klientin zu bearbeitende Task identisch sein mit einer explizit formulierten Fragestellung für das Coaching, sie kann sich von dieser aber auch unterscheiden oder sie konkretisieren. So kann ein erster Schritt der Aufgabenerledigung sein, zunächst die Situation zu beschreiben, auf die die Klientin sich bezieht, und dort insbesondere das Unklare herauszuarbeiten. Darauf aufbauend kann die Klientin ihre komplexen 
inneren Reaktionen und Konstruktionen, die im Zusammenhang mit der Problembeschreibung auftreten, explorieren und nach den jeweiligen Bedeutungen suchen.

Im Zuge einer Klärung bzw. des Findens eines Lösungsansatzes für das Unklare stellt sich dann bei der Klientin irgendeine Art von Erleichterung ein (Rice \& Saperia, 1984, S. 39). Diese gilt es wahrzunehmen als Marker für eine gefundene Antwort.

Das theoretische Modell lässt sie wie folgt darstellen:

\section{Theoretisches Modell}
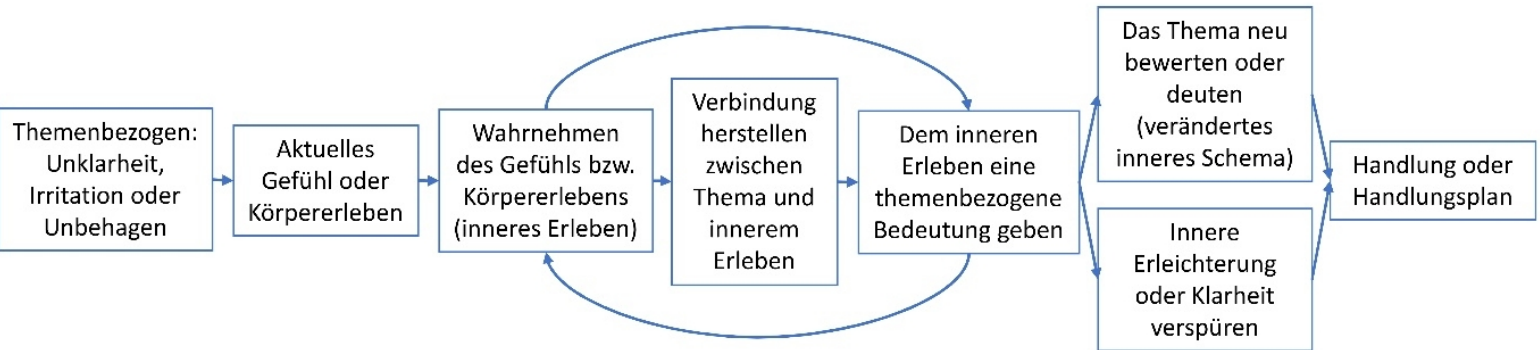

Abbildung 1: Theoretisches Aufgabenmodell im Veränderungsprozess

Am Anfang des Prozesses steht eine themenbezogene oder situationsbezogene Unklarheit, Irritation, oder ein Unbehagen. Beim Sprechen über die Unklarheit entsteht ein aktuelles Gefühl oder Körpererleben in dem Sinn, dass sich das bisherige Gefühl oder Körpererleben auf irgendeine Art und Weise verändert. Die Klientin wird sich dessen gewahr und stellt - implizit oder explizit - eine Verbindung zwischen dem Thema bzw. der Situation und ihrem inneren Erleben her (felt referent, felt sense), verweilt also beim gefühlten Erleben und kann diesem eine erste Bedeutung für das bearbeitete Thema geben. Je nach Situation erfolgt dann die Wahrnehmung des inneren Erlebens und das Explizieren der Bedeutung des Erlebens in mehreren Schleifen, bis die Klientin zu einem veränderten inneren Schema gelangt, das eine Neubewertung des Themas ermöglicht und mit einer inneren Erleichterung oder neu erlangten Klarheit einhergeht (felt shift). Dies ermöglicht eine spezifische, auf das Thema bezogene Handlung oder einen auf das Thema bezogenen Handlungsplan, den die Klientin als adäquat im Sinn einer Übereinstimmung mit ihrem inneren Erleben empfindet.

\subsection{Empirische Task Analyse}

Die empirische Task-Analyse erfolgte auf Basis der Empfehlungen von Rice \& Saperia (1984), wobei die Entscheidung bei der Samplebildung zugunsten einer Kontrastierung (Pascual-Leone, 2009) getroffen wurde, um nicht nur Erkenntnisse über ein Idealmodell, sondern gegebenenfalls auch über Prozessabbrüche zu gewinnen. 


\subsubsection{Sampling}

Ziel des Sampling war, aus dem Material zunächst 3 idealtypische Sequenzen und anschließend 3 weniger erfolgreich verlaufende Sequenzen auszuwählen. Die Kriterien für die idealtypischen Sequenzen wurden aus den bereits beschriebenen Merkmalen des Task Markers und den ebenfalls beschriebenen Kontextbedingungen abgeleitet:

- Vorhandensein des Task Markers als Beginn eines Veränderungsprozesses: von der Klientin artikulierte Unklarheit, Unsicherheit oder Irritation in Verbindung mit Gefühlen oder Fragen der Haltung oder Motivation; Klärungsanliegen bzw. direkt oder indirekt geäußerter Veränderungswunsch

- Moment der Verlangsamung oder der Verlagerung des Gesprächsprozesses, Suche nach passenden Worten

- Experiencing: Erkennbar anhand der Experiencing-Scala: EXP Stufe 4 oder mehr

- Felt Shift oder anderweitiger signifikanter Veränderungsmoment im Sinn der Ausbildung alternativer funktionaler Deutungs- und Handlungsmuster als Zeichen für den erfolgreichen Abschluss des Prozesses.

Als Kontrast dienen solche Prozesse, bei denen der Task Marker und das Experiencing zwar erkennbar sind, sich aber kein Felt Shift oder anderweitiger signifikanter Veränderungsmoment als Marker für den erfolgreichen Abschluss des Prozesses finden lässt.

Konkret erfolgte das Sampling wie folgt:

- Durchsehen der Memos mit der Fragestellung, welche Coachingsitzungen möglicherweise idealtypische oder kontrastierende Episoden im Sinn der Fragestellung enthalten könnten

- Wenn sich aus den Memos Hinweise auf das Vorhandensein idealtypischer oder kontrastierender Prozesse ableiten ließen: Lesen der Transkripte der entsprechenden Coachingsitzungen und auszugsweise nochmaliges Hören der Audiographien; gegebenenfalls Markieren von Task Marker und Task Finish

- Abbilden der für die Analyse in Frage kommenden Episoden in Form wortgetreuer Transkriptauszüge und Markieren und Begründen der Task Marker, Rating der Experiencing-Stufen in den Transkriptauszügen, Markieren und Begründen des Endes der Aufgabenerledigung in den Transkriptauszügen. Falls die Aufgabe nicht erfolgreich bearbeitet wurde, wurde als Ende der Aufgabenerledigung der Zeitpunkt festgelegt, zu dem sich Coach und Coachee einem neuen Gesprächsthema zuwenden

- Rice \& Saperia (1984, S. 30) und Rice \& Greenberg, (1983, S. 13) führen aus, dass die Task Analyse sich primär auf das Klientinnenverhalten fokussieren sollte, so dass zu überlegen war, inwieweit die Coachaussagen überhaupt in den Transkriptauszügen zu berücksichtigen waren. Ebenfalls war zu überlegen, inwieweit an dieser Stelle dem Beispiel von Wegener (2019, S. 113) zu folgen war, der die 
Transkriptpassagen reduzierte auf das unmittelbar für die Analyse relevante Kommunikationsgeschehen. An dieser Stelle entschied der Autor, die Transkriptauszüge zunächst vollständig abzubilden, und auch den Kontext der relevanten Passagen zu erfassen. So wurden bei der Abbildung der einzelnen Episoden auch jeweils 10 Minuten vor Beginn des Task Markers und nach dem Ende der Aufgabenerledigung in den Transkriptauszug aufgenommen, und das Kommunikationsverhalten von Coach und Coachee wurde vollständig wiedergegeben. Grund für diese Entscheidung war, dass in diesem frühen Stadium der Analyse eine Selektion augenscheinlich relevanter Passagen noch nicht möglich war und eine entsprechende Kürzung möglicherweise zu einer unnötigen Limitierung der Erkenntnisse geführt hätte. Um den Passagen eine gewisse Gliederung und Übersichtlichkeit zu geben, wurden die Task Marker und die Marker für den Abschluss der Aufgabenerledigung ebenso hervorgehoben wie diejenigen Klientinnenaussagen, die für das Rating des Experiencing von besonderer Bedeutung waren. Sonstige Hervorhebungen des Original-Transkripts wie markierte Anonymisierungen und Markierungen unverständlicher Aussagen wurden in den Transkriptauszügen entfernt, um keine Verwirrungen entstehen zu lassen

- Finale Auswahl der zu untersuchenden Episoden. Hierbei wurde als weiteres Auswahlkriterium die Unterschiedlichkeit der Fälle berücksichtigt. So wurde darauf geachtet, dass jeweils die drei als idealtypisch erachteten Episoden und die drei kontrastierenden Episoden auf Gesprächen unterschiedlicher Coachs und unterschiedlicher Coachees basierten, um die Erkenntnisse nicht vom Verarbeitungsschema einer einzelnen Coachee oder dem methodischen Vorgehen einer einzelnen Coach abhängig zu machen.

Die einzelnen Schritte des Sampling erfolgten iterativ anhand von weiteren Memos zu den einzelnen Coachingsitzungen, bis letzten Endes eine Priorisierung für drei idealtypische und drei kontrastierende Sitzungen vorgenommen wurde. Die Memos und Überlegungen zur Priorisierung wurden in einer eigenen Übersicht festgehalten (sh. Anlage 53: Sample-Bildung). So entstand folgende Auswahl:

Erste idealtypische Episode (2017_1805_01\#1):

- Dauer der Episode: 9 Minuten (Zeitstempel \#00:55:32-6\# bis \#01:00:04-5\#)

- Die Klientin ist wütend auf bestimmte Personen, sie erlebt im Gespräch eine intensive Gefühlsregung, die sie auch körperlich spürt. Sie ist entsetzt, dass sie sich so viel hat bieten lassen und wünscht, dass das zukünftig nicht mehr vorkommen möge. Im weiteren Verarbeiten konzentriert sich die Klientin auf ihren inneren Bezugsrahmen und erarbeitet eine Verlagerung der gefühlten Bedeutung. Die gefundene veränderte Bedeutung verschafft ihr ein Aha-Erlebnis in Form einer Überraschung über sich selbst. Auf dieser Grundlage entstehen neue Motivlagen und Handlungsoptionen. Das Experiencing-Niveau erreicht zeitweise EXP Stufe 
6

- Differenzierte Begründung des Task Markers, Task Erledigung und Rating zum Experiencing siehe Anlage 54: Task Analyse Prozess 2017_1805_01\#1.

Zweite idealtypische Episode (2016_1119a_01\#8):

- $\quad$ Dauer der Episode: 36 Minuten (Zeitstempel \#00:18:44-8\# bis \#00:54:54-5\#)

- Die Klientin beschreibt eine Ungeduld und ein allgemeines Unwohlsein, bezogen auf ihren Arbeitsplatz. Sie kann das zunächst nicht näher einordnen, hat aber ein starkes Bedürfnis nach Veränderung. Im weiteren Beschreiben der Situation erarbeitet sie zunächst ein inneres Bild (,Eingesperrtsein“) und weiter das körperliche Empfinden von Gelähmtsein. Das Experiencing erreicht einen Spitzenwert von EXP Stufe 5, und im Verlauf des Prozesses erarbeitet die Klientin eine Lösung, die ihr eine gewisse Wahlfreiheit ermöglicht und mit einer Erleichterung einhergeht

- Differenzierte Begründung des Task Markers, Task Erledigung und Rating zum Experiencing: siehe Anlage 55: Task Analyse Prozess_2016_1119a_01\#8.

Dritte idealtypische Episode (2017_2119_03\#3):

- $\quad$ Dauer der Episode: 20 Minuten (Zeitstempel \#00:51:45\# bis \#01:21:43\#)

- Die Klientin erlebt gegensätzliche Anforderungen, nicht nur von Seiten ihres Umfelds, auch in sich selbst. Hierfür wünscht sie sich eine Lösung. In der Bearbeitung des Themas bezieht sie berufliche und private Lebensbereiche ein und exploriert ihr inneres Erleben unter Einbeziehung ihres Körperempfindens. Im Experiencing ist ein Spitzenwert EXP 7 zu beobachten. Der Felt Shift entfaltet sich Schritt für Schritt, bis sie am Ende über die vor ihr liegende Perspektive sagen kann: „es gefällt mir“, auch wenn noch Unsicherheitsfaktoren bestehen bleiben, die mit einer gewisse Schwere einhergehen

- Differenzierte Begründung des Task Markers, Task Erledigung und Rating zum Experiencing siehe Anlage 56: Task Analyse Prozess_2017_2119_03\#3.

Erste kontrastierende Episode (2016_1119a_01\#1):

- $\quad$ Dauer der Episode: 17 Minuten (Zeitstempel \#00:34:31-1\# bis \#00:51:31-7\#)

- Die Klientin benennt eine Schwierigkeit im Umgang mit Mitarbeitenden. Sie erarbeitet im Rahmen ihres inneren Erlebens eine Metapher (,gefangen in einem kleinen Raum“) und setzt diese in Bezug zu ihrem beruflichen Handeln. Im Experiencing ist ein Spitzenwert EXP 5 zu beobachten, es entsteht ein ganz kurzer Moment der Energetisierung, ein Felt Shift deutet sich an, aber dann kommt es nicht zu einem vorwärts tragenden Prozess. Nach einigen Minuten kommt es zu einer Themenverlagerung, deren Bezug zur ursprünglichen Task nicht mehr erkennbar ist, so dass diese Themenverlagerung als Task Finish im Sinn einer nicht erfolgreich bewältigten Aufgabenbearbeitung gedeutet wird

- Differenzierte Begründung des Task Markers, Task Finish bzw. Themenwechsel 
und Rating zum Experiencing siehe Anlage 59: Task Analyse Prozess_2016_1119a_01\#1.

Zweite kontrastierende Episode (2015_2007_02\#1):

- Dauer der Episode: 28 Minuten (Zeitstempel \#00:48:11-7\# bis \#01:16:23-2\#

- Die Klientin benennt als Anliegen ein für sie schwieriges Thema, sie komme mit ihrem Leben nicht zurecht. Sie bezieht sich dabei zunächst auf private Situationen, später auch auf berufliche Situationen. Es bleibt für sie unklar, wie sie das einordnen und damit umgehen kann. Beim Erarbeiten des Themas erreicht sie die Stufe 4 auf der Experiencing-Skala und betont immer wieder, dass sie sich nicht richtig fühle. Ein Felt Shift ist nicht erkennbar, wohl aber eine Erleichterung nach dem Vorschlag des Coach, für die tiefere Bearbeitung eine Therapeutin aufzusuchen. Direkt im Anschluss an die geäußerte Erleichterung findet ein Themenwechsel statt, der als Task Finish betrachtet werden kann, ohne dass ein Veränderungsprozess stattgefunden hätte

- Differenzierte Begründung des Task Markers, Task Finish bzw. Themenwechsel und Rating zum Experiencing siehe Anlage 57: Task Analyse_2015_2007_02\#1.

Dritte kontrastierende Episode (2017_0118_01\#2):

- Dauer der Episode: 3 Minuten (Zeitstempel \#00:10:32-00\# bis \#00:13:48-0\#

- Die Klientin formuliert eine Unklarheit, verbunden mit ihrer Haltung und einem Unsicherheitserleben. Im Anschluss an den Task Marker erfolgt allerdings keine experienzielle Erarbeitung. Stattdessen gelangt die Klientin auf anderem Weg nach wenigen Minuten zu einer Handlungsoption und erarbeitet hierzu verschiedene Aspekte. Eine weitere Verbindung dieser Handlungsoption zur ursprünglichen Task wird im Gesprächsverlauf nicht mehr hergestellt und ist auch implizit nicht zu erkennen. Experiencing im Sinn einer erlebensmäßigen Verarbeitung ab EXP Stufe 4 ist in der Episode und auch im Anschluss an die Episode nicht zu erkennen, so dass diese Episode nicht die für diese Task Analyse bzw. eine gelungene Aufgabenbewältigung notwendige Kontextbedingung erfüllt. Im Sinn einer Kontrastierung könnte sie trotzdem zum Erkenntnisgewinn beitragen und soll daher hier ebenfalls Berücksichtigung finden

- Differenzierte Begründung des Task Markers, Task Finish bzw. Themen-wechsel und Rating zum Experiencing siehe Anlage 58: Task Analyse_2017_0118_01\#2.

\subsubsection{Codierung der Episoden}

Bei der Codierung wurde darauf geachtet, die Codes primär anhand des klientinnenseitigen Kommunikationsverhaltens zuzuweisen. Coachäußerungen wurden nicht als Kategorie berücksichtigt, sondern lediglich als Kontext, um Klientinnenäußerungen verstehen und einordnen zu können. Scheinbar nicht unmittelbar aufgabenbezogene Kommunikationselemente der 
Coachee wurden bei der Codierung ebenfalls berücksichtigt, um einem möglichen Informationsverlust oder gar einer Präjudiz und unangemessenen Selektion von Kommunikationsbeiträgen entgegen zu wirken.

In der ersten Etappe wurden zunächst die drei idealtypischen Prozesse untersucht. Dazu wurde in einem ersten Schritt eine Codierung der Gesprächsepisoden auf der Grundlage des theoretischen Modells vorgenommen. Folgende Codes wurden aus dem theoretischen Modell abgeleitet:

\begin{tabular}{|l|l|}
\hline $\begin{array}{l}\text { Hauptkategorie } \\
\text { auf Basis des theoretischen Modells }\end{array}$ & Unterkategorie \\
\hline Thema / Situation & Situationsbeschreibung \\
\hline & Irritation / Unbehagen \\
\hline & Herausarbeiten der Unklarheit \\
\hline Aktuelles Gefühl / Körpererleben & Fragestellung \\
\hline Wahrnehmung inneren Erlebens (Selbsterleben / \\
referring inward)
\end{tabular}

Tabelle 5: Codes auf Grundlage des theoretischen Modells 
Nach einer ersten Codierung auf Basis des theoretischen Modells wurden weitere Codes gebildet, um die modellbasierte Codierung zu überprüfen bzw. zu ergänzen. Hierfür wurden entsprechend der Empfehlung von Greenberg (2007) zunächst Makrokategorien gebildet, um verschiedene Stränge innerhalb des Veränderungsprozesses abzubilden. Da es beim Thema dieser Arbeit einerseits um das Selbsterleben und andererseits um die Wahrnehmung des arbeitsbezogenen Kontexts (Personen, Situationen) geht, erfolgte die Codierung anhand dieser beiden Makrokategorien:

- Selbsterleben im Sinn des Experiencing, der erlebten Emotionen, des körperlich spürbaren Erlebens und der Selbstwahrnehmung

- Kontextbezug: Bezugnahme auf Szenen, Situationen und Personen.

Bei dieser weiteren Codierung wurde zunächst ein beschreibendes Codierungsniveau gewählt, das sich nah am Kommunikationsgeschehen orientierte. Diese Kategorien wurden dann in einem dritten Durchgang ergänzt um Kategorien, die Greenberg (ebd.) als „higherlevel-categories" bezeichnet, solche Codes also, die das Geschehen auf einem höheren Abstrahierungsniveau beschreiben. Ziel dieser weiteren Codierungsdurchgänge war die Erweiterung / Ergänzung oder auch Differenzierung der anhand des theoretischen Modells entwickelten Codes.

So entstand für jede der Episoden eine Übersicht, bestehend aus Transkript, Marker für den Task-Beginn und das Task-Ende, EXP-Stufe und den jeweiligen Codierungen als Grundlage für die Erstellung der empirischen Modelle zu den einzelnen Prozessen (sh. Anlagen 54-56: Task Analysen zu den Prozessen 2017_1805_01\#1, 2016_1119a_01\#8 und 2017_2119_03\#3).

Ergänzend wurden die Higher-Level-Codierungen der Episoden in einer gemeinsamen Tabelle notiert, um einen späteren Vergleich zu ermöglichen (sh. Anlage 60: Empirische Analyse, Higher-Level-Codierungen, Abschnitt 1.1).

In der zweiten Etappe wurden die drei kontrastierenden Prozesse untersucht, das Procedere war dabei identisch mit dem Vorgehen der ersten Etappe: Zunächst erfolgte eine Codierung auf Basis des theoretischen Modells anhand der bereits oben beschriebenen Codierungen. Anschließend wurden weitere Codes gebildet, analog zur Codierung der idealtypischen Beispiele entlang der beiden Makrokategorien Selbsterleben und Kontextbezug und sowohl auf einem beschreibenden Codierungsniveau als auch als abstrahierende Higher-Level-Kategorien (sh. Anlagen 57-59: Task Analysen zu den Prozessen 2015_2007_02\#1, 2016_1119a_01\#1 und 2017_0118_01\#2). Die Codierung der drei kontrastierenden Episoden erfolgte unabhängig und auch in einem gewissen zeitlichen Abstand zu der Codierung der drei idealtypischen Episoden, um dem Material möglichst unvoreingenommen begegnen zu können. Analog zum bereits bei den idealtypischen Episoden umgesetzten Vorgehen wurden auch die Higher-Level-Codes der kontrastierenden Episoden in einer gemeinsamen Übersicht zusammengefasst, um sie für einen späteren Vergleich verfügbar zu haben (sh. Anlage 60: Empirische Analyse, Higher-Level-Codierungen, Abschnitt 1.2). 


\subsubsection{Empirische Modellbildung}

Um die Codes in ein empirisches Modell überführen zu können, wurde im nächsten Schritt eine Verdichtung vorgenommen, indem das Transkript auf diejenigen Passagen reduziert wurde, die für das Rating oder die Codierung von Bedeutung waren. Als bedeutsam wurden all diejenigen Passagen erachtet, denen Einschätzungen oder Kommentare zur Task, zum Experiencing oder zur Codierung zugeordnet waren (sh. jeweils Abschnitt 2 der Task Analysen zu den Prozessen 2017_1805_01\#1; 2016_1119a_01\#8; 2017_2119_03\#3; 2015_2007_02\#1; 2016_-1119a_01\#1; 2017_0118_01\#2). Auf Basis dieser Verdichtung wurde in der Zusammenschau der einzelnen Stränge (EXP-Stufen, Codes auf Basis des theoretischen Modells und der beiden Makrokategorien Selbsterleben und Kontextbezug) ein Ablaufmodell in Textform beschrieben und gleichzeitig ein Diagramm entworfen, das die wichtigsten Prozesskomponenten darstellen sollte. Dabei wurde besonders geachtet auf:

- Abweichungen zwischen der Codierung auf Basis des theoretischen Modells und der weiteren Codierung anhand der beiden Makrokategorien

- Vergleich der beiden Veränderungsstränge bzw. Makrokategorien Selbsterleben und Kontextbezug

- Auffälligkeiten im Vergleich zwischen dem Rating der EXP-Stufen und den Kategorien.

Für die sechs untersuchten Episoden konnten so die im Folgenden dargestellten Modelle bzw. Prozessdiagramme (Abbildungen 2-7) erarbeitet werden. Dort, wo in den Diagrammen zwischen einzelnen Prozesselementen ein spezifischer Zusammenhang verdeutlicht werden sollte, wurde dies anhand von Pfeilen dargestellt.

\subsubsection{Erste idealtypische Episode (2017_1805_01\#1) - empirisches Modell}

Die Klientin beschreibt eine Situation, die sie beschäftigt. Sie reflektiert dabei einerseits äußere Einflussfaktoren und andererseits, welche Nähe bzw. Distanz sie zu ihrem Umfeld einnimmt, oder allgemeiner gesagt, in welcher Beziehung sie zu ihrem Umfeld steht. Die Reflexion der bestimmenden Umfeldfaktoren bezieht sich sowohl auf gegenwärtige als auch zurückliegende Einflüsse, die aus dem Umfeld kommen. Das eigene Fühlen und Erleben wird in dieser Schilderung zwar einbezogen, es wird aber eher anhand von Verhaltensweisen oder in enger Verknüpfung mit Verhaltensweisen dargestellt (EXP 3).

Im weiteren Verlauf vertieft die Klientin die Situation und arbeitet die Differenzen zwischen dem Kontext einerseits, in diesem Fall problematische Interaktionen von Menschen in ihrem Umfeld, und dem Selbsterleben andererseits noch deutlicher heraus (EXP 4). Im Selbsterleben tauchen dabei zwei Stränge auf: zum einen ein Verantwortungsgefühl als Handlungsmotiv und zum anderen ein unangenehmes Körpererleben. Im weiteren Vertiefen der Situation in Verbindung mit dem Handlungsmotiv und dem Körpererleben wird eine Emotion wahrgenommen und aktuell erlebt (Wut). Gleichzeitig findet eine Bewertung oder Einordnung des Interaktionsverhaltens des Umfelds statt. Das Experiencing erreicht einen Spitzenwert EXP 
5. In dieser Sequenz zeigt sich auch der Task Marker, der zu Beginn der Situationsbeschreibung noch nicht klar erkennbar war. Die Unklarheit wird als Entscheidungsdilemma in Verbindung mit dem Selbsterleben beschrieben. Auf der Erlebensebene erarbeitet die Klientin eine weitere Differenzierung, hier in Form einer Metapher (,,aufplatzen vor Wut"), mit der sie die Auswirkung des inneren Erlebens (inneres Dilemma) auf das Handeln beschreibt. Das Verhalten des Umfelds ordnet sie weiter ein.

Es kommt zu einem weiteren Körpererleben. Dieses unterscheidet sich vom zuerst genannten Körpererleben dadurch, dass es neu und spontan auftritt, und dass die Klientin es in der aktuellen Situation sehr deutlich spürt. Sie nimmt diese Veränderung wahr und widmet ihr Aufmerksamkeit. Die Klientin erarbeitet die Bedeutung dieses Körpererlebens im Sinn eines Felt Sense, stellt also eine Verbindung zwischen der Situation und dem Erleben her (EXP 6). Sie ist überrascht von dieser Bedeutung und in gewisser Weise erleichtert, die Bedeutung erkannt zu haben (Felt Shift). Die weitere Auseinandersetzung findet quasi spiralförmig statt: Im Hinund Her-Pendeln zwischen Felt Sense, Felt Shift und der Wahrnehmung der Veränderung im inneren Erleben entsteht eine Neubewertung, in diesem Fall das Erleben von Souveränität, verbunden mit einer Energetisierung, Aktivierung eigener Ressourcen und einer neuen Zielformulierung. Insgesamt entsteht also Selbstwirksamkeit, und daraus entwickelt die Klientin einen inneren Handlungsplan und vertieft diesen, indem sie die Vorteile benennt.

Während in der vorherigen Sequenz die Aufmerksamkeit der Klientin primär beim Selbsterleben war, kommt nun wieder das Umfeld in den Blick: sie beschreibt die aus ihrer Sicht positive Bedeutung ihres veränderten Handlungsplans für ihr Umfeld. Damit ist die Task bearbeitet, das Experiencing verändert sich zu EXP 3, und es kommt zu einem Themenwechsel. Auffallend im Prozess ist, dass es eine Phase der Erarbeitung gibt, in der die Klientin beim Selbsterleben verweilt und die Wahrnehmung des Umfelds in den Hintergrund tritt. Erst am Ende des Veränderungsprozesses findet wieder eine verstärkte Bezugnahme auf das Umfeld statt. 


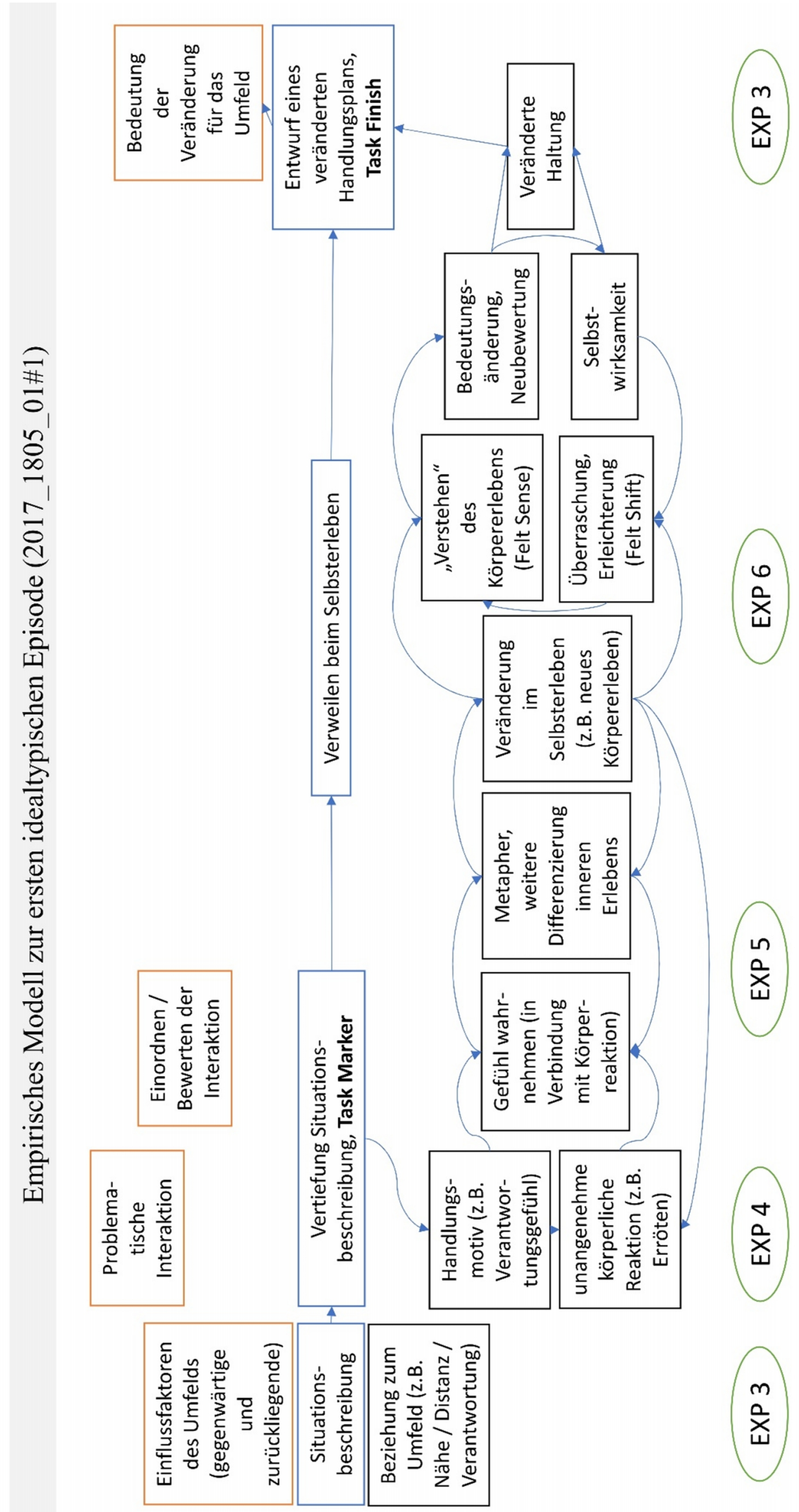

Abbildung 2: Empirisches Modell zur ersten idealtypischen Episode 
Im Blick auf den Strang des Selbsterlebens wurde bei der Erarbeitung des Modells nicht explizit differenziert zwischen dem Beschreiben eines zurückliegenden Selbsterlebens bzw. einer zurückliegenden Körperreaktion und dem aktuellen Erleben. Grund hierfür war, dass diese Differenzierung auch aus dem Transkript in Verbindung mit der Audiographie nicht eindeutig erkennbar war, weil auch Schilderungen, die sich eigentlich auf die Vergangenheit bezogen, sehr präsent vorgetragen wurden, so dass die zeitliche Distanz in den Hintergrund rückte.

Ergänzend schien es so, dass die erlebensmäßige Verarbeitung in einer Art Kreisform von statten ging, so dass mehrere Aspekte der Körper- oder Gefühlswahrnehmung in gegenseitiger Wechselwirkung verarbeitet wurden. Im Diagramm wurde diese Kreisform durch geschwungene Pfeile dargestellt, die sowohl von rechts nach links zeigen, als auch von links nach rechts, von oben nach unten und umgekehrt. Damit sollen die Wechselwirkungen dargestellt werden, so dass der Prozess an dieser Stelle nicht linear als von rechts nach links ablaufend verstanden werden soll, sondern eher als sich zirkulär entfaltend.

\subsubsection{Zweite idealtypische Episode (2016_1119a_01\#8) - empirisches Modell}

Die Klientin beschreibt detailliert eine berufliche Situation, die sie persönlich sachlich und emotional involviert. Sie erlebt ein aus ihrer Sicht problematisches Interaktionsverhalten ihres Umfelds, und bei sich selbst beschreibt sie Unbehagen und ein Gefühl von Wut gegenüber dem Umfeld. Damit einher gehend erlebt sie ein kognitiv-affektives Dilemma, nämlich einerseits den Wunsch, sich in ihrem Verhalten zu beherrschen, und andererseits die Realität, dass eine rationale Verhaltenssteuerung nicht gelingt. Fühlen und Erleben wird in enger Verknüpfung mit Verhaltensweisen beschrieben (EXP 3).

Im weiteren Verlauf vertieft die Klientin die Situationsbeschreibung in Verbindung mit der Wahrnehmung ihres inneren Erlebens und ihres Unbehagens. Sie beschreibt ein weiteres Gefühl (Frustration) und wiederholt die Wahrnehmung, dass ihr eigenes Verhalten derzeit stärker von Affekten gesteuert ist als von rationalen Überlegungen. Das erlebt sie auch körperlich, eigene Grenzen sind für sie deutlich spürbar. Das Verhalten des Umfelds bewertet sie negativ, von den Anforderungen bzw. Erwartungen des Umfelds distanziert sie sich. Die Klientin formuliert dabei einen impliziten Veränderungswunsch, den sie später noch einmal aufgreift. Das kann als Vorläufer zum eigentlichen Task Marker gewertet werden (im Modell als PreTask-Marker bezeichnet).

Die Klientin vertieft ihr Selbsterleben und verbindet dieses mit dem Thema bzw. der Situation. Die Experiencing-Stufe verändert sich von EXP 3 hin zu EXP 4, innere Vorgänge werden differenziert, und die Klientin expliziert zunehmend die Bedeutung des Erlebens. Sie sieht sich selbst fremdbestimmt und reflektiert dazu ihr eigenes Engagement bzw. ihre Motivation. Im weiteren Verlauf zeigt sie sich irritiert über sich selbst und artikuliert das Bedürfnis nach einer Veränderung (Task Marker). Das Experiencing bleibt bei EXP 4, und gleichzeitig sucht sie im Verhalten des Umfelds nach Erklärungen für das eigene Erleben. Beim Selbsterleben 
dominieren Orientierungslosigkeit und Ratlosigkeit, die Wahrnehmung verändert sich im Sinn einer zunehmenden emotionalen Belastung und zu einem unangenehmen Körpererleben. Die Schilderung bezieht sich auf etwas, was kurze Zeit zurück liegt, aber gleichzeitig sehr präsent wirkt und quasi aktuell erlebt wird.

Die Klientin verweilt weiter bei ihrem Erleben. Das Umfeld ist dabei nicht vollständig ausgeblendet, sondern wird punktuell zum Impulsgeber für das eigene Erleben. Ihre inneren Vorgänge erhalten eine zunehmende Bedeutung (Felt Sense), sie verarbeitet diese weiter, differenziert sie, formuliert klar ihr Körpergefühl (Gelähmtsein) und findet eine Metapher (Eingesperrtsein) für ihr Erleben (EXP 5). Wiederholt spürt sie ihre Begrenzung (Kraftlosigkeit, Ratlosigkeit).

Eine Veränderung im Erleben liegt darin, dass sie die emotionale Belastung noch deutlicher expliziert, und dass sie noch weniger positive Zukunftsbilder und Ressourcen erkennen kann. Das Verweilen geschieht zirkulär, so dass es mehrere Momente des Körpererlebens, des Erlebens von Orientierungs- und Ratlosigkeit und des Eindrucks von Fremdbestimmung und Unsicherheit gibt. Die veränderte, verdüsterte Wahrnehmung mündet in ein starkes Gefühl (Wut), und dieses wiederum führt dazu, dass die Klientin ihre eigenen Bedürfnisse und Ressourcen erarbeitet. Sie verweilt bei der Beschreibung dessen, wie sie sich selbst bei der Arbeit erlebt und was sie dort benötigt. Dies führt zu einem fürsorglichen Blick auf sich selbst (Selbstfürsorge), und sie bezieht sich dabei auf ihren inneren Bezugsrahmen (EXP 4). Nun kann sie ihre Ressourcen aktivieren. An die Stelle des Gefühls der Fremdbestimmung tritt die Wahrnehmung, selbstbestimmt etwas aussuchen zu können. Hoffnung keimt auf, die Stimmung verändert sich positiv, eine Erleichterung im Sinn eines Felt Shift stellt sich ein. Gleichzeitig erreicht die Klientin den Zeitpunkt, an dem die Aufgabe erfolgreich bewältigt ist (Task Finish): sie hat ihre Handlungsfähigkeit, die vorher ,gelähmt“" und ,eingesperrt“ war, wiederhergestellt. In den folgenden Minuten vertieft und erweitert sie die Möglichkeit ihrer Handlungsspielräume unter Bezugnahme auf ihr Umfeld, nimmt einen Abgleich mit den Rahmenbedingungen des Umfelds wahr, und bei sich selbst erlebt sie jetzt Stimmigkeit. 


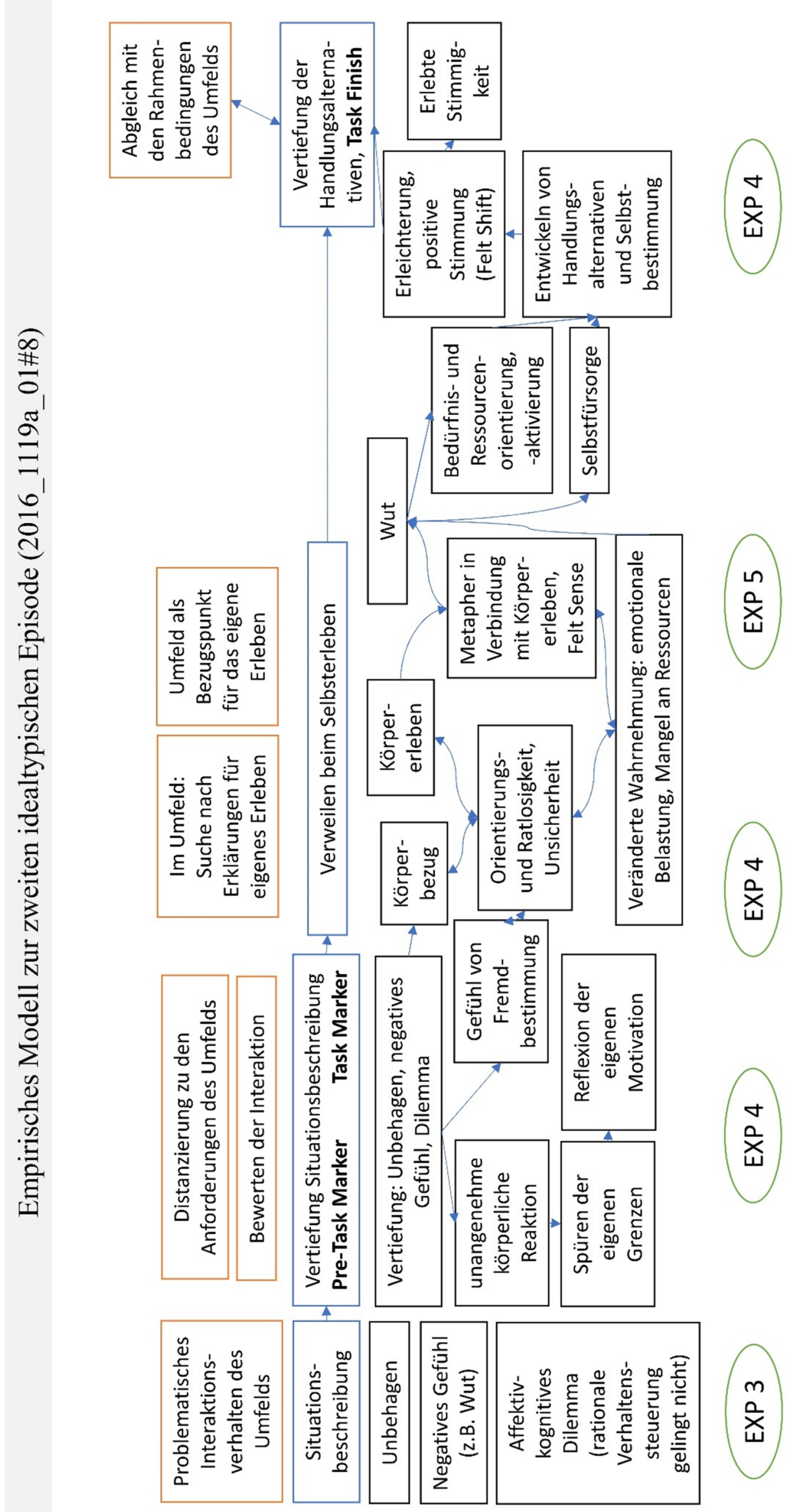

Abbildung 3: Empirisches Modell zur zweiten idealtypischen Episode 


\subsubsection{Dritte idealtypische Episode (2017_2119_03\#3)-empirisches Modell}

Die Klientin beschreibt zusammenfassend, wie sie ihre Gesamtsituation erlebt: sie hat Sorge um sich selbst und ist unsicher, wie sie die Situation einordnen soll. Ihr Umfeld erlebt sie als anspruchsvoll. Sie ist unzufrieden mit dem Verhalten des Umfelds und wünscht sich eine Veränderung in der Interaktion zwischen dem Umfeld und sich selbst. Ihre persönliche Rolle wird dabei deutlich, wobei persönliche Gefühle und Reaktionen eher abstrakt geschildert werden, so dass das Experiencing auf Stufe 2 einzuschätzen ist (EXP 2). Im weiteren Verlauf konkretisiert sie ihre Unzufriedenheit mit dem Verhalten des Umfelds und ordnet dieses als problematisch ein.

Der Veränderungswunsch steht in einem Zusammenhang mit dem inneren Erleben: die Klientin stellt eine Verbindung her zwischen ihrem Selbsterleben und der Situation bzw. ihrem Thema und beschreibt gegensätzliche Anforderungen, die von anderen und von ihr selbst an sie gestellt werden. Diese führen zu einem inneren Dilemma, weil sie sich nicht zwischen den unterschiedlichen Anforderungen entscheiden möchte oder kann, und insgesamt zu einem Erleben von Belastetsein. Sie reflektiert auch ihre eigene Haltung dabei und erlebt sich selbst als ungeduldig.

Dann reflektiert die Klientin ihre eigene berufsbiographische Entwicklung. Sie sucht insgesamt Orientierung, wie sie die Situation einordnen kann und hält Ausschau nach etwas, was ihr helfen, also eine Veränderung bringen würde (Task Marker). Das Zuwenden zum inneren Erleben und das Herstellen einer Verbindung zwischen Situation und Erleben deutet auf Experiencing-Stufe 4 (EXP 4) hin.

Im weiteren Verlauf zirkuliert die Klientin in mehreren Wiederholungsschleifen zwischen ihrem inneren Dilemma, ihrem Belastetsein, der Ungeduld und der Suche nach Lösungen bzw. Orientierung. Parallel dazu erfolgt eine weitere Bezugnahme auf das Umfeld, sowohl in Form der Bewertung des Verhaltens des Umfelds als auch in der Form, dass sie sich von den Anforderungen des Umfelds distanziert. Auch diese Bezugnahme geschieht in mehreren Durchläufen in einer Art Zirkel, in dem sie mehrfach ihre eigene Positionierung zum Umfeld klärt und dessen Verhalten einordnet.

In einer nächsten Sequenz bringt die Klientin weitere biographische Elemente ein und erforscht in diesem Zusammenhang ihre eigenen Bedürfnisse und auch ihre eigenen Ressourcen im Sinn guter Erfahrungen, auf die sie zurückgreifen kann. Die Klientin kann dann in ihrem Inneren aktuell vorhandene emotionale Aspekte wahrnehmen und auch differenzieren (EXP 5). Die Differenzierung besteht darin, dass sie einerseits angesichts der Komplexität des Themas ein Gefühl von Überfordertsein beschreibt, und andererseits auch zunehmend eigene Ressourcen erkennt und gleichzeitig ihre eigene Motivation und ihre Werte reflektiert, indem sie sich fragt, welchen bzw. wessen Anforderungen sie Folge leisten möchte. Sie spürt dem nach, was ihr gut tun würde und exploriert damit weiter ihre eigenen Bedürfnisse.

Die Erwartungen des Umfelds stehen in ihrer Wahrnehmung im Kontrast zur eigenen Lebensorientierung und werden sowohl in der biographischen Reflexion als auch in der aktuellen 
Situation als fordernd wahrgenommen. Innerlich geht die Klientin auf Distanz zu diesen Forderungen.

Sie verweilt weiter bei der Verbindung zwischen ihrem Selbsterleben und ihrem Thema. Im wiederholten Nachspüren dessen, was ihr guttun würde, und im Rückgriff auf ihre eigenen Ressourcen entsteht ein Körpererleben, und damit einhergehend eine Verlagerung weg vom Überforderungserleben hin zum differenzierten Wahrnehmen von stärkenden und förderlichen Aspekten. Sie erarbeitet die Bedeutung des Körpererlebens im Sinn eines Felt Sense und nimmt das Körpererleben als vertrauenswürdige Quelle wahr (EXP 7). Daraus ergibt sich für die Klientin eine klarere Erkenntnis, sie beginnt, ihr Thema neu zu bewerten.

Im Rahmen der Neubewertung findet sie ein Bild als Metapher für einen Lösungsschritt, der sich für sie abzeichnet. Sie gleicht ihre Bedürfnisse und ihre Lösungsidee nochmals mit dem Verhalten des Umfelds ab und stellt fest, dass die Unsicherheit noch nicht komplett gewichen ist.

In weiteren Schritten benennt sie vertiefend ihre jetzt veränderte und geklärte Motivation im Zusammenhang mit der Neubewertung des Themas. Auch wenn die Unsicherheit sie weiter begleitet, formuliert sie erneut eigene Bedürfnisse, nimmt eine aktive Rolle ein und entwickelt Handlungsoptionen für nächste Schritte. Sie spürt dabei eine zunehmende Stimmigkeit und beschreibt, dass die anfänglich formulierte Sorge bzw. Angst nicht mehr vorhanden ist und der positive Ausblick ihre Stimmung auch positiv verändert (Felt Shift, EXP 6), so dass die gestellte Aufgabe des Umgangs mit der Sorge und dem erlebten Dilemma bewältigt ist (Task Finish). Vor diesem Hintergrund arbeitet sie weiter an ihrem Handlungsplan, vertieft die Überlegungen dazu und erarbeitet, wie sie mit der verbleibenden Restunsicherheit umgehen möchte. 


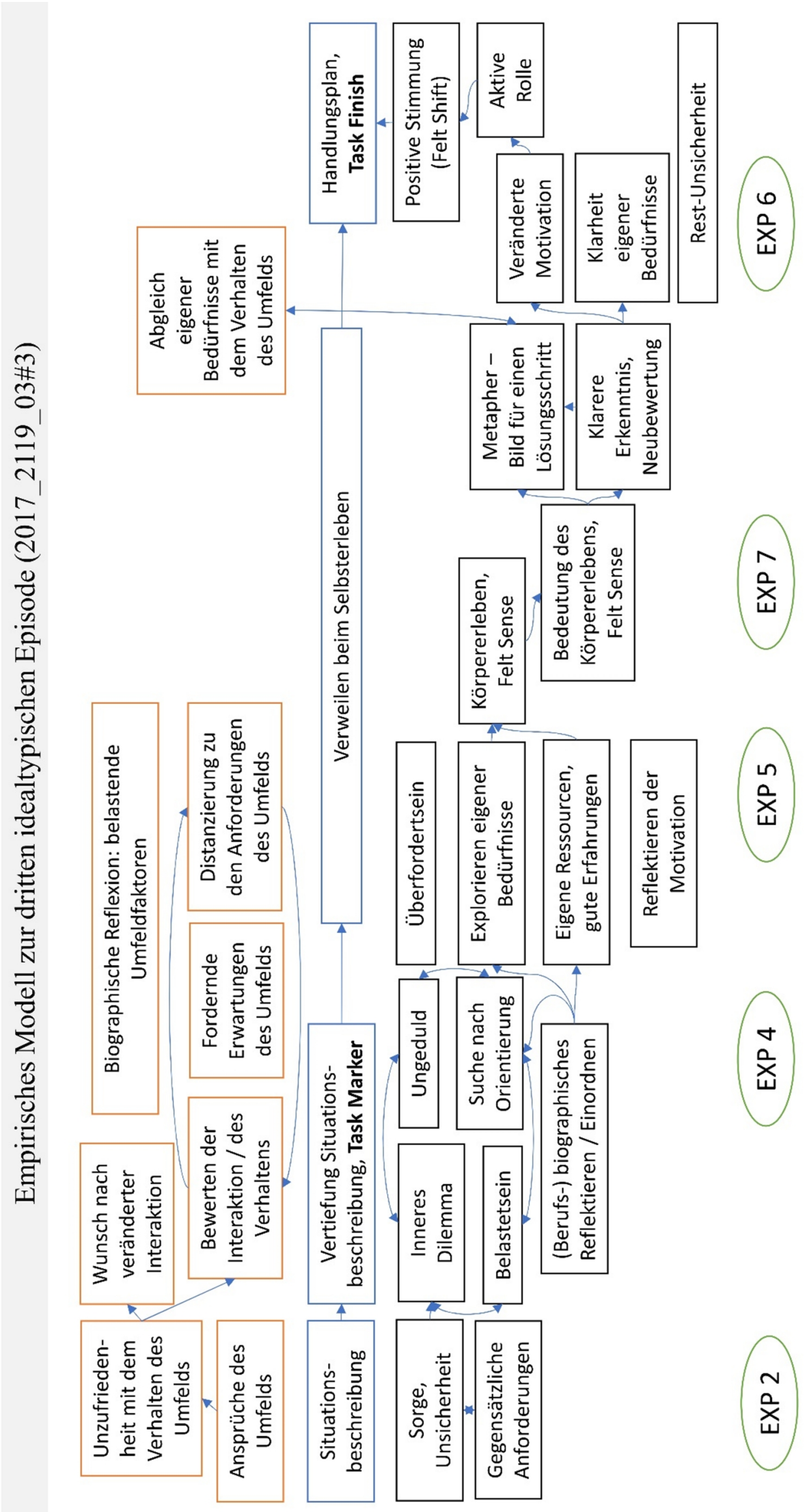

Abbildung 4: Empirisches Modell zur dritten idealtypischen Episode 


\subsubsection{Erste kontrastierende Episode (2016 1119a 01\#1) - empirisches Modell}

Die Klientin beschreibt eine Situation in ihrem Arbeitsfeld, die ihr Mühe bereitet. Ihre persönliche Rolle ist dabei erkennbar, der Bericht bezieht sich zunächst weniger auf das eigene Fühlen als mehr auf die Darstellung des Sachverhalts und die Deutung des Verhaltens der anderen an der Situation beteiligten Personen (EXP 2). Mit dem Interaktionsverhalten dieser Personen beschäftigt sich die Klientin intensiv, sie beschreibt es als problematisch und konflikthaft. Für sich selbst empfindet sie, dass das Verhalten des Umfelds Druck entstehen lässt. Über ihre eigene Reaktion auf das Verhalten des Umfelds ist die Klientin irritiert, sie kann dazu wenig sagen und beschreibt auf Nachfrage eine Unklarheit bezüglich ihrer inneren Vorgänge.

Im weiteren Verlauf beschäftigt sie sich wieder intensiv mit dem Interaktionsverhalten des Umfelds und beschreibt dieses detailliert. Sie sieht sich selbst dabei in der Verantwortung für das Umfeld, auch in dem Sinn, dass sie gewisse Handlungen vornehmen sollte. Es entsteht eine gewisse Handlungserwartung gegenüber sich selbst.

Die Klientin schildert weiter beispielhaft eine Situation, bei der sie ihr Verhalten änderte, ohne aber eine anschließende Verbesserung der Situation und ihres Belastungserlebens zu erkennen. Sie fühlt eine hohe emotionale Belastung, beschreibt diese als Schwere und als große innere Hürde. In dieser Phase, in der sie sich auf ihr Inneres konzentriert und Fragen ihrer Motivation reflektiert, bringt sie ihr Fühlen und ihr Verhalten in Bezug zueinander, der innere Bezugsrahmen wird zu Grundlage der Mitteilung (EXP 4). Bezogen auf die beschriebene innere Hürde und die damit einhergehenden Fragen würde sie gerne mehr Klarheit erhalten (Task Marker). Bezogen auf ihr Umfeld beschreibt sie Anforderungen, in diesem Fall ein emotional unter Druck setzendes Verhalten des Umfelds, und für sich selbst formuliert sie gleichzeitig Mitgefühl mit dem Umfeld.

Eine gewisse innere Unklarheit bleibt weiter bestehen, und zwar in dem Sinn, dass das innere Erleben sich nicht weiter eröffnet und die Klientin keine Bedeutung ihres Selbsterlebens explizieren kann. Einerseits nimmt sie ihr Selbsterleben zwar deutlich wahr (EXP 4), formuliert die Erlebensinhalte aber nur begrenzt, beschäftigt sich parallel mit dem Umfeld und beschreibt dieses als Ursache für das eigene Unwohlbefinden.

Es bleibt dabei, dass die Bedeutung des inneren Erlebens sich nicht erschließt. Die Klientin setzt sich weiter mit der Situation des Umfelds auseinander, sie identifiziert sich mit dem Erleben des Umfelds. Die Exploration des Erlebens des Umfelds überlagert die Exploration des eigenen Erlebens, so dass das Experiencing-Niveau zwischenzeitlich auf Stufe 2 sinkt (EXP 2). Sie nimmt Rücksicht auf die Befindlichkeit des Umfelds, erlebt sich selbst emotional belastet und erkennt einen Mangel an eigener Handlungsfähigkeit. Dann konzentriert sie sich wieder stärker auf ihren inneren Bezugsrahmen (EXP 4), erarbeitet die Bedeutung des inneren Erlebens bis hin zu einer Differenzierung (Spitzenwert EXP 5), und es entsteht ein Veränderungswunsch, der kurzzeitig Energie und Ressourcen aktiviert. Hierbei verweilt sie für einen Augenblick, denkt über eine Erfahrung mit einer Veränderung ihres Handelns nach, und es 
entsteht ein zweiter kurzer Moment der Energetisierung und inneren Veränderung im Sinn einer Erleichterung, die dann aber nicht weiter voranträgt.

Beim Herstellen der Verbindung zwischen Thema und Selbsterleben beschreibt die Klientin sich zwar als in ihrer Rolle funktionierend und agierend, es bleibt aber bei einem Belastungserleben in Verbindung mit einer fehlenden Problemlösung. Wiederholt kehrt sie zurück zu der erlebten Handlungsunfähigkeit, und im Selbsterleben dominiert die emotionale Belastung. In den weiteren Überlegungen wendet sie sich wieder dem Umfeld zu und beschreibt dort weitere problematische Aspekte. Dabei tauchen neue, bisher nicht berichtete Facetten der Unklarheit auf, und es kommt zu einer Themenverlagerung. Da die Klientin später nicht mehr zu der anfänglich benannten Unklarheit bzw. Task zurückkehrt, wird diese Themenverlagerung als Task Finish im Sinn nicht erfolgreicher Aufgabenerledigung gewertet. Im weiteren Verlauf entwickelt sich das Experiencing dann hin zu Stufe 2 (EXP 2). 


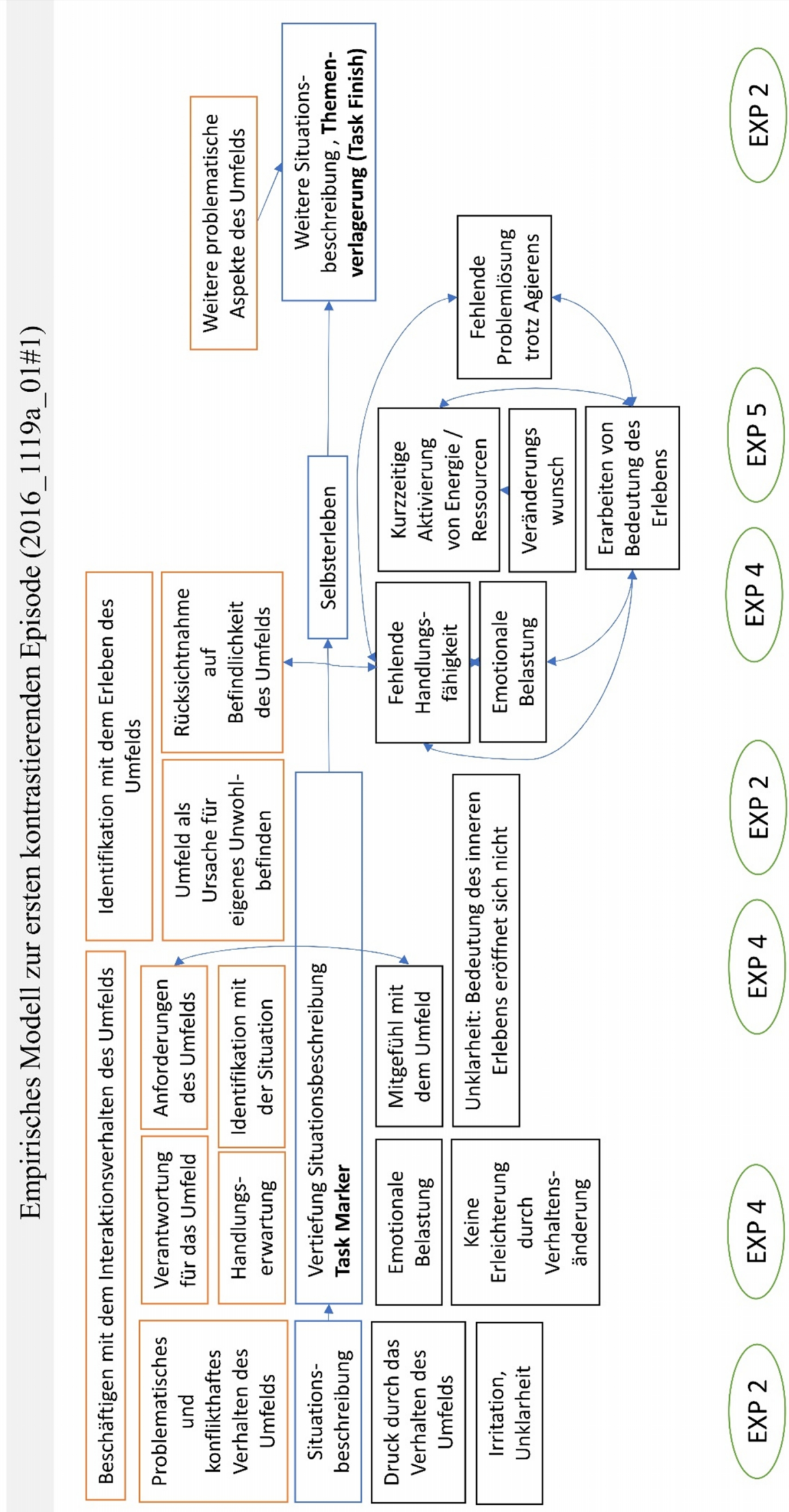

Abbildung 5: Empirisches Modell zur ersten kontrastierenden Episode 


\subsubsection{Zweite kontrastierende Episode (2015_2007_02\#1) - empirisches Modell}

Die Klientin thematisiert ein Problem, das sie als sehr schwierig erlebt und das nicht nur arbeitsbezogene, sondern in weiten Teilen auch außerberufliche Lebensbereiche tangiert. Sie beschreibt die Situation und verbindet damit eine hohe emotionale Belastung, ein großes inneres Unbehagen und den Wunsch nach Veränderung (Task Marker). Das Unklare besteht darin, dass die Klientin mit der Situation nicht gut zurechtkommt und keinen Ansatzpunkt zu einem Umgang damit oder zu einer Veränderung findet.

In der weiteren Vertiefung der Situationsbeschreibung benennt die Klientin belastende Aspekte ihres Umfelds. Sie stellt eine Verbindung zum Selbsterleben her. Dabei schildert sie ihre Gefühle und emotionale Belastung in einer eher allgemeinen Art, ohne dass ein momentanes Erleben und Beteiligtsein erkennbar wäre und ohne dass sie ihr Selbsterleben in diesem Moment weiter bearbeiten würde (EXP 3).

Die Klientin verweilt dann beim Explorieren ihrer emotionalen Belastung, und im Bemühen, ihren inneren Bezugsrahmen zu erforschen, erreicht sie ein zwischenzeitliches Experiencingniveau der Stufe 4 (EXP 4). Sie nimmt persönliche Defizite bei sich wahr und beschreibt diese. Anschließend verändert sich das Experiencing wieder dahingehend, dass das Fühlen und Erleben zwar beschrieben wird, aber keine weitere Erarbeitung oder Differenzierung stattfindet (EXP 3).

In der weiteren Beschreibung thematisiert die Klientin problematisches Verhalten des Umfelds, und bei sich nimmt sie eine zunehmende Kraftlosigkeit wahr. Sie schätzt sich als hilfsbedürftig ein und wiederholt ihren Veränderungswunsch. Das Umfeld nimmt sie dabei nicht als Ressource wahr.

Im nächsten Schritt versucht sie, sich vom belastenden emotionalen Selbsterleben zu distanzieren. Sie bezieht gefühlte Bedeutungen eher begrenzt in ihre Schilderungen ein und berichtet über das innere Erleben wie von einem äußeren Bezugspunkt aus (EXP 2).

Anschließend greift die Klientin einen Vorschlag des Coachs auf, der ihr eine Handlungsalternative im Sinn eines anderen Orts zur Bearbeitung ihrer emotionalen Belastung empfiehlt. Daraus entwickelt sie einen Plan für einen nächsten Handlungsschritt und erlebt eine Erleichterung. Diese Erleichterung ist allerdings nicht als Task Finish im Sinn einer erfolgreich bewältigten Aufgabenerledigung zu werten, denn die Erleichterung entsteht zum einen nicht aus einem experienziellen Prozess heraus, und zum anderen hat die Klientin ihre Task hier nicht vertiefend bearbeitet, sondern die Bearbeitung an einen anderen Ort verlagert.

Im Anschluss kommt es bis zum Ende der Coachingsitzung zu einem Themenwechsel, der als Task Finish gewertet wird. Das Experiencingniveau der Klientin bleibt weiter auf Stufe 2 (EXP 2). 


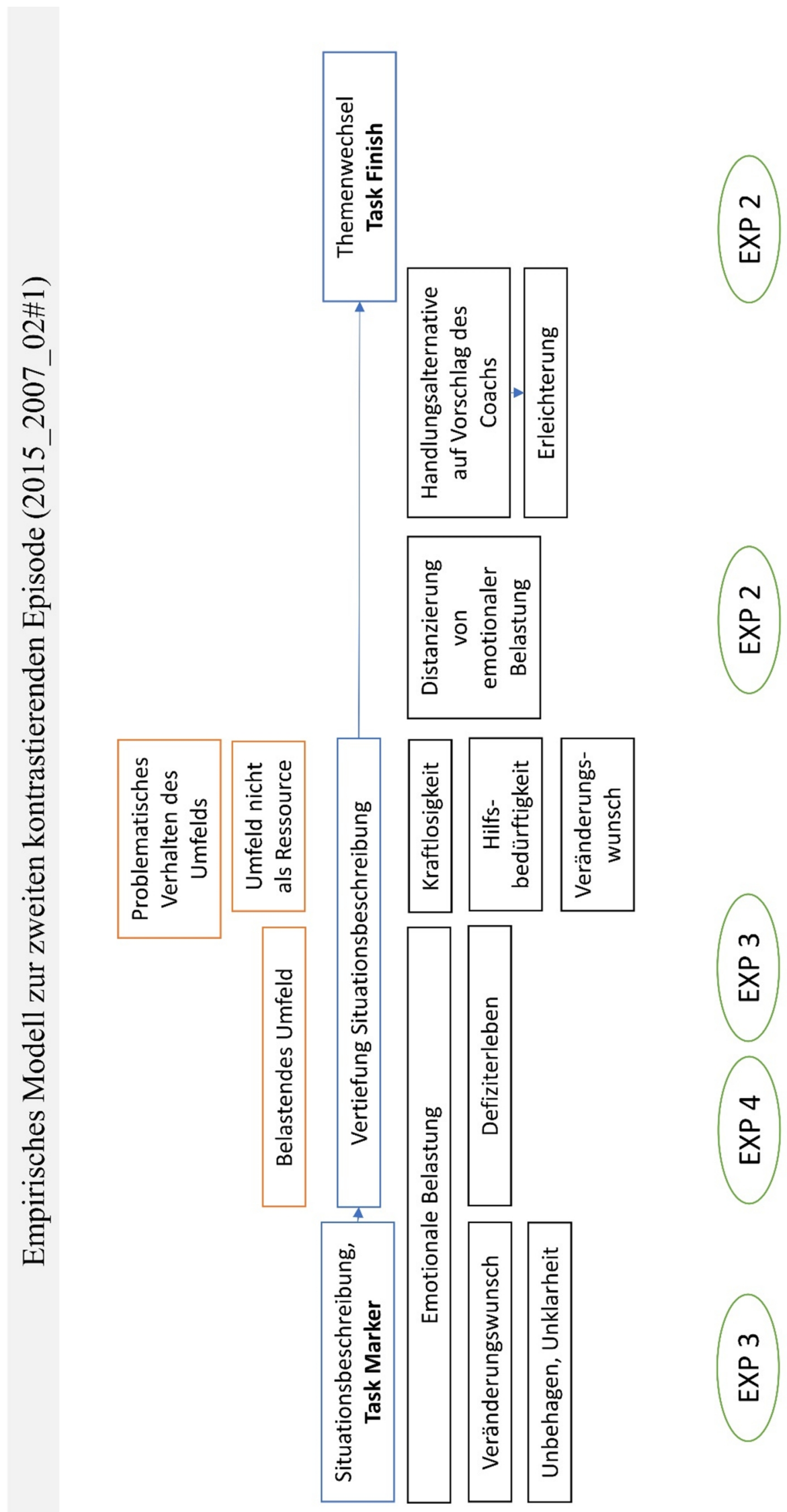

Abbildung 6: Empirisches Modell zur zweiten kontrastierenden Episode 


\subsubsection{Dritte kontrastierende Episode (2017_0118_01\#2) - empirisches Modell}

Die Klientin beschreibt eine Situation, bei der sie sich in einem Entscheidungsdilemma befindet. Es ist ihr unklar, wie sie sich ihrem Umfeld gegenüber positionieren soll. In gewisser Weise ist sie irritiert und unsicher, welche Folgen ihr eigenes Verhalten haben wird. Ihr eigenes Interesse am Thema ist erkennbar, auf ihr Fühlen und Erleben nimmt sie dabei indi-

$$
\text { rekt Bezug (EXP 2). }
$$

Hinsichtlich des Umfelds gibt es nicht nur die Unklarheit der eigenen Positionierung, die Klientin weiß auch nicht, wie sich das Umfeld zu ihr selbst positioniert. Die Klientin wendet sich ihrem Selbsterleben insofern zu, als dass sie eine Verbindung zwischen der Situation und ihrer Haltung herstellt und daraus eine Fragestellung ableitet (Spitzenwert EXP 3). Die Fragestellung bezieht sich auf ihr Dilemma und markiert gleichzeitig die Task. Die Unklarheit wird weiter herausgearbeitet und mit der eigenen Unsicherheit und einem Veränderungswunsch in Verbindung gebracht. Bezogen auf das Umfeld bleibt es bei der bereits beschriebenen Unsicherheit darüber, wie sich dieses zur eigenen Person der Klientin positioniert.

Bei der Suche nach einer Lösung entwickelt die Klientin schnell die Idee, die eigene Position als Referenzpunkt zu nutzen und dem Umfeld gegenüber eigene Vorstellungen zu formulieren. Daraus erarbeitet sie einen Lösungsansatz im Sinn einer Handlungsoption. Diese konkretisiert sie dann mit Hilfe der Coach, das Experiencing ist zu diesem Zeitpunkt auf Stufe 2 einzuschätzen (EXP 2). Die anfänglich angesprochene Unsicherheit und Unklarheit ist nicht mehr direkt Thema, es wird im Gesprächsverlauf aber auch nicht erkennbar, dass die Unklarheit wirklich aufgelöst ist.

Da die Klientin eine Themenverlagerung vornimmt und die anfänglich thematisierte Unklarheit nicht mehr direkt aufgreift, wird dieser Zeitpunkt als Task Finish im Sinn eines Themenwechsels und einer nicht finalisierten Aufgabenbearbeitung gewertet. 

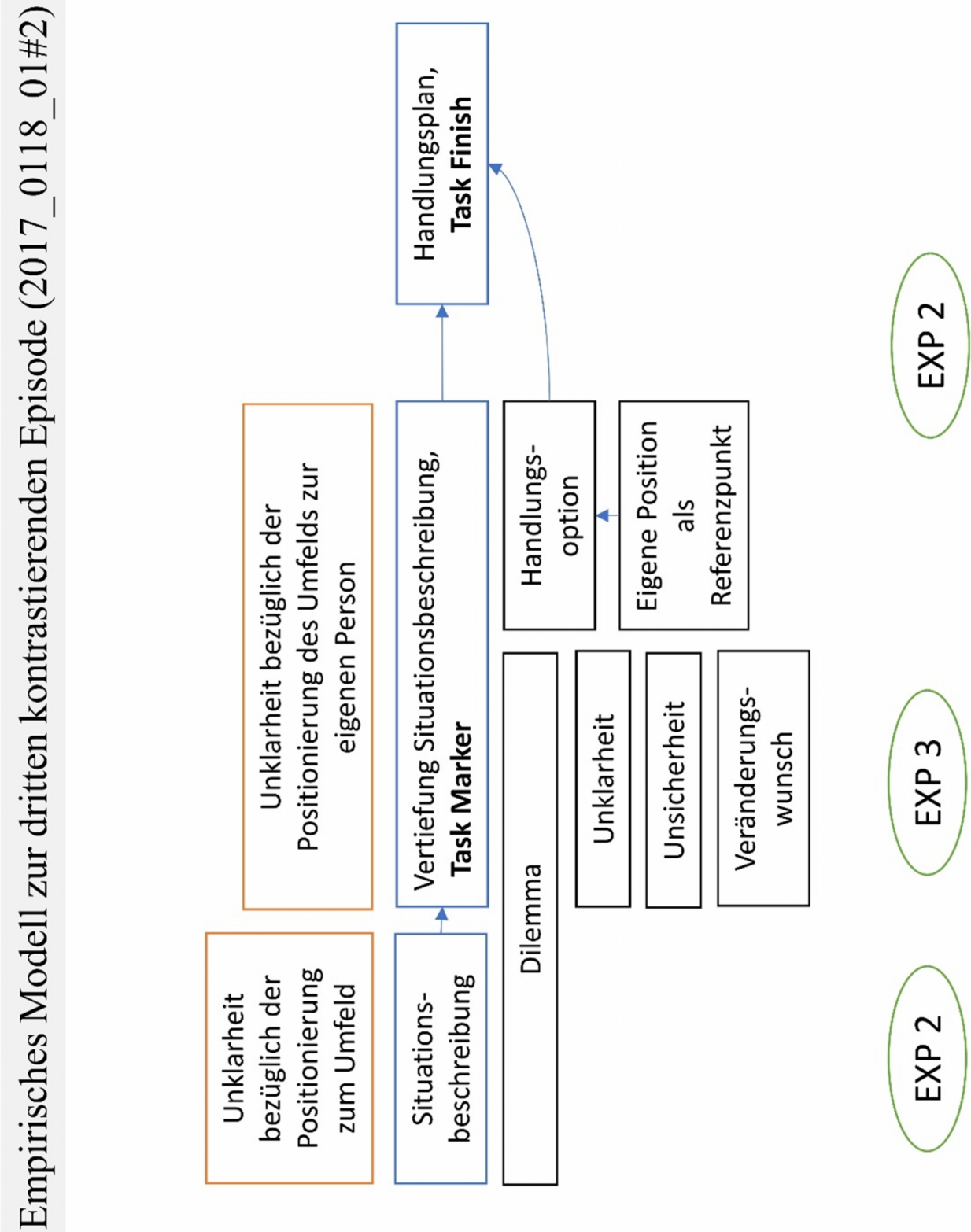

Abbildung 7: Empirisches Modell zur dritten kontrastierenden Episode

\subsubsection{Rekonstruktion des empirisch-theoretischen Modells}

Ziel dieses Schritts ist, als Synthese zwischen dem theoretischen Modell und den empirischen Befunden ein „client operating model“ zu rekonstruieren, das die Veränderungsmechanismen und das Experiencing der Klientin möglichst präzise beschreibt (Rice \& Saperia, 1984, S. 58).

\subsubsection{Kernelemente des empirischen Befunds}

Für einen Abgleich der empirischen Erkenntnisse mit dem theoretischen Modell wurden zunächst in den Higher-Level-Codes der idealtypischen Episoden übereinstimmende Merkmale herausgearbeitet, um nach Möglichkeit Kernelemente des Prozesses zu identifizieren. Dazu 
wurde zum einen im Strang Selbsterleben und zum anderen im Strang Kontextbezug nach inhaltlich übereinstimmenden Kategorien gesucht, und die im Rahmen der Codierung erstellte Übersicht der Higher-Level-Codes (sh. Anlage 60: Empirische Analyse, Higher-Level-Codierungen) wurde verdichtet auf diese übereinstimmenden Kategorien und jeweils für die Makrokategorie Selbsterleben und die Makrokategorie Kontextbezug in eine Tabelle übertragen. Doppelnennungen wurden zur Verdeutlichung beibehalten. In einer ergänzenden Spalte wurde dann eine Beschreibung für das jeweilige übereinstimmende Merkmal formuliert:

\begin{tabular}{|c|c|c|c|}
\hline \multicolumn{3}{|c|}{ Makrokategorie Selbsterleben } & \multirow{2}{*}{$\begin{array}{l}\text { Übereinstimmendes } \\
\text { Merkmal des Prozesses }\end{array}$} \\
\hline $\begin{array}{l}\text { Episode } \\
2017 \_1805 \_01 \# 1\end{array}$ & $\begin{array}{l}\text { Episode } \\
\text { 2016_1119a_01\#8 }\end{array}$ & $\begin{array}{l}\text { Episode } \\
2017 \_2119 \_03 \# 3\end{array}$ & \\
\hline \multirow[t]{2}{*}{ Inneres Dilemma } & $\begin{array}{l}\text { kognitiv-affektives } \\
\text { Dilemma }\end{array}$ & Inneres Dilemma & \multirow{3}{*}{$\begin{array}{l}\text { Spannungserleben: } \\
\text { Erleben einer gewissen } \\
\text { Spannung, die sowohl af- } \\
\text { fektiv als auch kognitiv } \\
\text { wahrgenommen wird und } \\
\text { sich auf ein Missverhält- } \\
\text { nis zwischen der eigenen } \\
\text { Person und dem Umfeld } \\
\text { oder ein Missverhältnis } \\
\text { unterschiedlicher Motiva- } \\
\text { tionsstränge innerhalb der } \\
\text { eigenen Person bezieht }\end{array}$} \\
\hline & Inneres Dilemma & Inneres Dilemma & \\
\hline $\begin{array}{l}\text { Nähe-Distanz- } \\
\text { Einordnung, be- } \\
\text { zogen auf das } \\
\text { Umfeld }\end{array}$ & & $\begin{array}{l}\text { Erleben von An- } \\
\text { derssein }\end{array}$ & \\
\hline $\begin{array}{l}\text { Erleben eines star- } \\
\text { ken Gefühls }\end{array}$ & $\begin{array}{l}\text { Erleben eines star- } \\
\text { ken Gefühls }\end{array}$ & Ungeduld & \multirow{7}{*}{$\begin{array}{l}\text { Emotionale Belastung: } \\
\text { Wahrnehmen eines star- } \\
\text { ken Gefühls, das mit dem } \\
\text { Erleben von Beeinträch- } \\
\text { tigtsein einher geht und } \\
\text { nicht ignoriert werden } \\
\text { kann }\end{array}$} \\
\hline $\begin{array}{l}\text { Vertiefte Be- } \\
\text { schreibung des } \\
\text { Gefühlserlebens }\end{array}$ & $\begin{array}{l}\text { Wahrnehmung: Af- } \\
\text { fekt bestimmt das } \\
\text { eigene Verhalten }\end{array}$ & $\begin{array}{l}\text { Unsicherheitserle- } \\
\text { ben }\end{array}$ & \\
\hline \multirow[t]{5}{*}{$\begin{array}{l}\text { Erleben von Be- } \\
\text { grenzung }\end{array}$} & $\begin{array}{ll}\text { Spüren eigener } \\
\text { Grenzen }\end{array}$ & $\begin{array}{l}\text { Wahrnehmen emo- } \\
\text { tionaler Belastung }\end{array}$ & \\
\hline & $\begin{array}{l}\text { Erleben von Fremd- } \\
\text { bestimmung }\end{array}$ & $\begin{array}{l}\text { Gefühl von Über- } \\
\text { forderung }\end{array}$ & \\
\hline & Ratlosigkeit & & \\
\hline & Ratlosigkeit & & \\
\hline & Kraftlosigkeit & & \\
\hline
\end{tabular}




\begin{tabular}{|c|c|c|c|}
\hline Körperreaktion & Körpererleben & $\begin{array}{l}\text { Körpererleben und } \\
\text { dessen Bedeutung } \\
\text { wahrnehmen }\end{array}$ & \multirow{6}{*}{$\begin{array}{l}\text { Körpererleben: } \\
\text { Spüren einer körperlichen } \\
\text { Reaktion, die als bedeut- } \\
\text { sam für die Situation ver- } \\
\text { standen wird }\end{array}$} \\
\hline $\begin{array}{l}\text { In-Beziehung- } \\
\text { Setzen von Kör- } \\
\text { perreaktion und } \\
\text { Gefühl }\end{array}$ & $\begin{array}{l}\text { Körperbezug ohne } \\
\text { direktes Körperer- } \\
\text { leben }\end{array}$ & & \\
\hline $\begin{array}{l}\text { Aktuelles Körper- } \\
\text { erleben }\end{array}$ & $\begin{array}{l}\text { körperliches Be- } \\
\text { dürfnis }\end{array}$ & & \\
\hline $\begin{array}{l}\text { In-Beziehung- } \\
\text { Setzen von Kör- } \\
\text { perreaktion und } \\
\text { Gefühl }\end{array}$ & $\begin{array}{l}\text { körperliches Be- } \\
\text { dürfnis }\end{array}$ & & \\
\hline $\begin{array}{l}\text { Erkennen der Be- } \\
\text { deutung von Kör- } \\
\text { pererleben und } \\
\text { Gefühl }\end{array}$ & Körpererleben & & \\
\hline $\begin{array}{l}\text { Bedeutungsände- } \\
\text { rung (im Zusam- } \\
\text { menhang mit Kör- } \\
\text { pererleben) }\end{array}$ & Körpererleben & & \\
\hline $\begin{array}{l}\text { Metapher für Ge- } \\
\text { fühlserleben }\end{array}$ & & $\begin{array}{l}\text { Metapher für das } \\
\text { aktuelle Erleben }\end{array}$ & $\begin{array}{l}\text { Metapher: } \\
\text { Ein Bild oder ein Symbol } \\
\text { kann als ergänzende Ex- } \\
\text { plikation des Selbsterle- } \\
\text { bens dienen }\end{array}$ \\
\hline \multirow[t]{3}{*}{ Handlungsmotiv } & $\begin{array}{l}\text { Reflexion des eige- } \\
\text { nen Engagements } \\
\text { bzw. der eigenen } \\
\text { Motivation }\end{array}$ & $\begin{array}{l}\text { Reflexion der eige- } \\
\text { nen Motivation }\end{array}$ & \multirow{3}{*}{$\begin{array}{l}\text { Reflexion der Motivation: } \\
\text { Die Motivation, die das } \\
\text { eigene Handeln leitet, } \\
\text { wird reflektiert. Dazu } \\
\text { können auch biographi- } \\
\text { sche Einflüsse gehören, } \\
\text { die das aktuelle Erleben } \\
\text { und Verhalten prägen }\end{array}$} \\
\hline & $\begin{array}{l}\text { Biographische Re- } \\
\text { flexion des aktuel- } \\
\text { len Erlebens }\end{array}$ & $\begin{array}{l}\text { Biographische Re- } \\
\text { flexion des aktuel- } \\
\text { len Erlebens }\end{array}$ & \\
\hline & & $\begin{array}{l}\text { Biographische Re- } \\
\text { flexion des aktuel- } \\
\text { len Erlebens }\end{array}$ & \\
\hline
\end{tabular}




\begin{tabular}{|c|c|c|c|}
\hline \multirow[t]{6}{*}{$\begin{array}{l}\text { Aktivierung eige- } \\
\text { ner Ressourcen }\end{array}$} & Selbstfürsorge & $\begin{array}{l}\text { Beschreiben eige- } \\
\text { ner Werte }\end{array}$ & \multirow{6}{*}{$\begin{array}{l}\text { Bedürfnisorientierte Res- } \\
\text { sourcenaktivierung: } \\
\text { Entdecken eigener und in } \\
\text { Verbindung mit eigenen } \\
\text { Bedürfnissen stehender } \\
\text { Ressourcen }\end{array}$} \\
\hline & $\begin{array}{l}\text { Veränderte Wahr- } \\
\text { nehmung der eige- } \\
\text { nen Ressourcen }\end{array}$ & $\begin{array}{l}\text { Explorieren eigener } \\
\text { Bedürfnisse }\end{array}$ & \\
\hline & $\begin{array}{l}\text { Erlebensorientierte } \\
\text { Reflexion eigener } \\
\text { Bedürfnisse und } \\
\text { Ressourcen }\end{array}$ & $\begin{array}{l}\text { Nachspüren: was } \\
\text { würde mir guttun? }\end{array}$ & \\
\hline & $\begin{array}{l}\text { Weitere erlebens- } \\
\text { orientierte Refle- } \\
\text { xion eigener Be- } \\
\text { dürfnisse und Res- } \\
\text { sourcen }\end{array}$ & $\begin{array}{l}\text { Formulierung eige- } \\
\text { ner Bedürfnisse }\end{array}$ & \\
\hline & & $\begin{array}{l}\text { Entdecken } \\
\text { eigener Ressourcen }\end{array}$ & \\
\hline & & $\begin{array}{l}\text { eigene Ressourcen } \\
\text { entdecken }\end{array}$ & \\
\hline $\begin{array}{l}\text { Zusammenfassen } \\
\text { des Aha-Erlebnis- } \\
\text { ses }\end{array}$ & $\begin{array}{l}\text { Erleben eines star- } \\
\text { ken (positiven) Ge- } \\
\text { fühls }\end{array}$ & Aktive Rolle & $\begin{array}{l}\text { Aktivierung von Energie: } \\
\text { Spürbare Zunahme des } \\
\text { Energieniveaus und der }\end{array}$ \\
\hline \multirow[t]{3}{*}{ Energetisierung } & $\begin{array}{l}\text { positive Verände- } \\
\text { rung der Stimmung }\end{array}$ & $\begin{array}{l}\text { positive Verände- } \\
\text { rung der Stimmung }\end{array}$ & \multirow[t]{3}{*}{ Zuversicht } \\
\hline & $\begin{array}{l}\text { positive Verände- } \\
\text { rung der Stimmung }\end{array}$ & $\begin{array}{l}\text { positiv veränderte } \\
\text { Stimmung }\end{array}$ & \\
\hline & & Positiver Ausblick & \\
\hline Zielformulierung & $\begin{array}{l}\text { Erarbeiten einer } \\
\text { Handlungsoption }\end{array}$ & $\begin{array}{l}\text { Handlungsoptionen } \\
\text { entwickeln }\end{array}$ & \multirow{3}{*}{$\begin{array}{l}\text { Entwickeln einer Hand- } \\
\text { lungsoption: } \\
\text { Benennen von Hand- } \\
\text { lungsschritten, gegebe- } \\
\text { nenfalls auch in Verbin- } \\
\text { dung mit einer veränder- } \\
\text { ten eigenen Haltung }\end{array}$} \\
\hline $\begin{array}{l}\text { „Inneren Hand- } \\
\text { lungsplan“ } \quad \text { ent- } \\
\text { werfen }\end{array}$ & & & \\
\hline $\begin{array}{ll}\text { inneren } & \text { Hand- } \\
\text { lungsplan } & \text { vertie- } \\
\text { fen } & \end{array}$ & & & \\
\hline
\end{tabular}

Tabelle 6: Übereinstimmende Higher-Level-Kategorien der Makrokategorie Selbsterleben in den idealtypischen Episoden 


\begin{tabular}{|c|c|c|c|}
\hline \multicolumn{3}{|c|}{ Makrokategorie Kontextbezug } & \multirow{2}{*}{$\begin{array}{l}\text { Übereinstimmendes } \\
\text { Merkmal des Prozesses }\end{array}$} \\
\hline $\begin{array}{l}\text { Episode } \\
2017 \_1805 \_01 \# 1\end{array}$ & $\begin{array}{l}\text { Episode } \\
\text { 2016_1119a_01\#8 }\end{array}$ & $\begin{array}{l}\text { Episode } \\
2017 \_2119 \_03 \# 3\end{array}$ & \\
\hline $\begin{array}{l}\text { Reflexion äußerer } \\
\text { Einflussfaktoren }\end{array}$ & $\begin{array}{l}\text { problematisches In- } \\
\text { teraktionsverhalten } \\
\text { des Umfelds }\end{array}$ & $\begin{array}{l}\text { Ansprüche des } \\
\text { Umfelds }\end{array}$ & \multirow{3}{*}{$\begin{array}{l}\text { Wahrnehmung problema- } \\
\text { tischer Interaktion des } \\
\text { Umfelds: } \\
\text { Das eigene Erleben wird } \\
\text { negativ beeinflusst durch } \\
\text { das Verhalten des Um- } \\
\text { felds }\end{array}$} \\
\hline $\begin{array}{l}\text { Wahrnehmung } \\
\text { pro-blematischen } \\
\text { Kommunikations- } \\
\text { verhaltens }\end{array}$ & & $\begin{array}{l}\text { Unzufriedenheit mit } \\
\text { dem Verhalten des } \\
\text { Umfelds }\end{array}$ & \\
\hline $\begin{array}{l}\text { Beschreibung des } \\
\text { Verhaltens der an- } \\
\text { deren }\end{array}$ & & $\begin{array}{l}\text { problematisches In- } \\
\text { teraktionsverhalten } \\
\text { des Umfelds }\end{array}$ & \\
\hline \multirow[t]{4}{*}{$\begin{array}{l}\text { Beurteilung des } \\
\text { Verhaltens der an- } \\
\text { deren }\end{array}$} & $\begin{array}{l}\text { Bewerten des Ver- } \\
\text { haltens der anderen }\end{array}$ & $\begin{array}{l}\text { Distanzierung zu } \\
\text { den Anforderungen } \\
\text { des Umfelds }\end{array}$ & \multirow{4}{*}{$\begin{array}{l}\text { Distanzierung zum Um- } \\
\text { feld: } \\
\text { Herausarbeiten der eige- } \\
\text { nen Positionierung im } \\
\text { Kontrast zum Umfeld }\end{array}$} \\
\hline & $\begin{array}{l}\text { Distanzierung zu } \\
\text { den Anforderungen } \\
\text { des Umfelds }\end{array}$ & $\begin{array}{l}\text { Bewertung des Ver- } \\
\text { haltens der anderen }\end{array}$ & \\
\hline & $\begin{array}{l}\text { Bewertung des } \\
\text { Verhaltens der an- } \\
\text { deren }\end{array}$ & $\begin{array}{l}\text { Distanzierung zu } \\
\text { den Anforderungen } \\
\text { des Umfelds }\end{array}$ & \\
\hline & $\begin{array}{l}\text { Distanzierung zu } \\
\text { den Anforderungen } \\
\text { des Umfelds }\end{array}$ & $\begin{array}{l}\text { Erleben von An- } \\
\text { derssein }\end{array}$ & \\
\hline \multirow[t]{2}{*}{$\begin{array}{l}\text { Anwendung des } \\
\text { veränderten } \\
\text { Handlungsplans } \\
\text { auf das Umfeld }\end{array}$} & $\begin{array}{l}\text { Abgleich des Hand- } \\
\text { lungsplans mit den } \\
\text { Rahmenbedingun- } \\
\text { gen des Umfelds }\end{array}$ & $\begin{array}{l}\text { Abgleich eigener } \\
\text { Bedürfnisse mit } \\
\text { dem Verhalten des } \\
\text { Umfelds }\end{array}$ & \multirow{2}{*}{$\begin{array}{l}\text { Anwendung der Verände- } \\
\text { rung auf das Umfeld: } \\
\text { Nach dem Erarbeiten ei- } \\
\text { ner Veränderung erfolgt } \\
\text { eine Hinwendung zum } \\
\text { Umfeld, um das Erarbei- } \\
\text { tete dort zur Anwendung } \\
\text { zu bringen }\end{array}$} \\
\hline & $\begin{array}{l}\text { Entdecken weiterer } \\
\text { Handlungsspiel- } \\
\text { räume }\end{array}$ & & \\
\hline
\end{tabular}

Tabelle 7: Übereinstimmende Higher-Level-Kategorien der Makrokategorie Kontextbezug in den idealtypischen Episoden 
Bei den kontrastierenden Episoden wurde ebenso nach übereinstimmenden Kategorien gesucht, wobei zunächst offen war, inwieweit hier übereinstimmende Codes als Kernmerkmale für weniger gelingende Prozesse gewertet werden können, oder inwieweit sie Aspekte darstellen, die bei der Bearbeitung von Unklarheit generell auftreten können, unabhängig davon, ob der Prozessverlauf als erfolgreich oder als weniger erfolgreich gewertet werden kann.

Folgende Merkmale konnten bei den kontrastierenden Episoden als übereinstimmend identifiziert werden:

\begin{tabular}{|c|c|c|c|}
\hline \multicolumn{3}{|c|}{ Makrokategorie Selbsterleben } & \multirow{2}{*}{$\begin{array}{l}\text { Übereinstimmendes } \\
\text { Merkmal des Prozesses }\end{array}$} \\
\hline $\begin{array}{l}\text { Episode } \\
\text { 2016_1119a_01\#1 }\end{array}$ & $\begin{array}{l}\text { Episode } \\
\text { 2015_2007_02\#1 }\end{array}$ & $\begin{array}{l}\text { Episode } \\
2017 \_0118 \_01 \# 2\end{array}$ & \\
\hline Druck & $\begin{array}{l}\text { Emotionale Belas- } \\
\text { tung }\end{array}$ & $\begin{array}{l}\text { Entscheidungs-Di- } \\
\text { lemma }\end{array}$ & \multirow{5}{*}{$\begin{array}{l}\text { Emotionales Belastungs- } \\
\text { erleben: } \\
\text { Ein Erleben von Belastet- } \\
\text { sein wird zum Ausdruck } \\
\text { gebracht, einhergehend } \\
\text { mit dem Wunsch nach ei- } \\
\text { ner Veränderung }\end{array}$} \\
\hline $\begin{array}{l}\text { Hohe emotionale } \\
\text { Belastung }\end{array}$ & Defiziterleben & Unsicherheit & \\
\hline \multirow[t]{3}{*}{$\begin{array}{l}\text { Emotionale Belas- } \\
\text { tung }\end{array}$} & Kraftlosigkeit & $\begin{array}{l}\text { Entscheidungsdi- } \\
\text { lemma }\end{array}$ & \\
\hline & $\begin{array}{l}\text { Erlebte Hilfsbe- } \\
\text { dürftigkeit }\end{array}$ & & \\
\hline & $\begin{array}{l}\text { Veränderungs- } \\
\text { wunsch }\end{array}$ & & \\
\hline
\end{tabular}

Tabelle 8: Übereinstimmende Higher-Level-Kategorien der Makrokategorie Selbsterleben in den kontrastierenden Episoden

\begin{tabular}{|c|c|c|c|}
\hline \multicolumn{3}{|c|}{ Makrokategorie Kontextbezug } & \multirow{2}{*}{$\begin{array}{l}\text { Übereinstimmendes } \\
\text { Merkmal des Prozesses }\end{array}$} \\
\hline $\begin{array}{l}\text { Episode } \\
\text { 2016_1119a_01\#1 }\end{array}$ & $\begin{array}{l}\text { Episode } \\
\text { 2015_2007_02\#1 }\end{array}$ & $\begin{array}{l}\text { Episode } \\
2017 \_0118 \_01 \# 2\end{array}$ & \\
\hline $\begin{array}{l}\text { Problematisches } \\
\text { Verhalten des } \\
\text { Umfelds }\end{array}$ & $\begin{array}{l}\text { Belastendes Um- } \\
\text { feld }\end{array}$ & $\begin{array}{l}\text { Unklarheit bezüg- } \\
\text { lich der Positionie- } \\
\text { rung zum Umfeld }\end{array}$ & $\begin{array}{l}\text { Wahrnehmung problema- } \\
\text { tischer Interaktion des } \\
\text { Umfelds: }\end{array}$ \\
\hline $\begin{array}{l}\text { Anforderungen } \\
\text { des Umfelds }\end{array}$ & $\begin{array}{l}\text { Problematisches } \\
\text { Verhalten des Um- } \\
\text { felds }\end{array}$ & $\begin{array}{l}\text { Unklarheit bezüg- } \\
\text { lich der Positionie- } \\
\text { rung des Umfelds } \\
\text { zur eigenen Person }\end{array}$ & $\begin{array}{l}\text { Das eigene Erleben wird } \\
\text { negativ beeinflusst durch } \\
\text { das Verhalten des Um- } \\
\text { felds }\end{array}$ \\
\hline
\end{tabular}




\begin{tabular}{|l|l|l|l|}
\hline $\begin{array}{l}\text { Umfeld als Ursa- } \\
\text { che für eigenes } \\
\text { Unwohlbefinden }\end{array}$ & Ressource & & \\
\hline $\begin{array}{l}\text { Weitere proble- } \\
\text { matische Aspekte } \\
\text { des Umfelds }\end{array}$ & & & \\
\hline
\end{tabular}

Tabelle 9: Übereinstimmende Higher-Level-Kategorien der Makrokategorie Kontextbezug in den kontrastierenden Episoden

Zusammenfassend konnten als übereinstimmende Merkmale der drei idealtypischen Episoden bezogen auf Veränderungsprozesse im Kontext von Experiencing folgende Aspekte festgehalten werden, wobei die Abfolge der einzelnen Prozessaspekte interindividuell unterschiedlich sein kann:

\begin{tabular}{|l|l|}
\hline Bezogen auf das Selbsterleben & Kontextbezogen \\
\hline $\begin{array}{l}\text { Spannungserleben: Erleben einer gewissen } \\
\text { Spannung, die sowohl affektiv als auch kogni- } \\
\text { tiv wahrgenommen wird und sich auf ein Miss- } \\
\text { verhältnis zwischen der eigenen Person und } \\
\text { dem Umfeld oder ein Missverhältnis unter- } \\
\text { schiedlicher Motivationsstränge innerhalb der } \\
\text { tird negativ beeinflusst durch das Verhal- } \\
\text { eigenen Person bezieht }\end{array}$ & \\
\hline $\begin{array}{l}\text { Emotionale Belastung: Wahrnehmen eines } \\
\text { starken Gefühls, das mit dem Erleben von Be- } \\
\text { einträchtigtsein einher geht und nicht ignoriert } \\
\text { werden kann }\end{array}$ & $\begin{array}{l}\text { Distanzierung zum Umfeld: Herausarbei- } \\
\text { ten der eigenen Positionierung im Kon- } \\
\text { trast zum Umfeld }\end{array}$ \\
\hline $\begin{array}{l}\text { Körpererleben: Spüren einer körperlichen Re- } \\
\text { aktion, die als bedeutsam für die Situation ver- } \\
\text { standen wird }\end{array}$ & \\
\hline $\begin{array}{l}\text { Metapher: Ein Bild oder ein Symbol kann als } \\
\text { ergänzende Explikation des Selbsterlebens } \\
\text { dienen }\end{array}$ & \\
\hline $\begin{array}{l}\text { Reflexion der Motivation: Die Motivation, die } \\
\text { das eigene Handeln leitet, wird reflektiert. } \\
\text { Dazu können auch biographische Einflüsse ge- } \\
\text { hören, die das aktuelle Erleben und Verhalten }\end{array}$ & \\
\hline
\end{tabular}




\begin{tabular}{|l|l|}
\hline $\begin{array}{l}\text { Bedürfnisorientierte Ressourcenaktivierung: } \\
\text { Entdecken eigener und in Verbindung mit ei- } \\
\text { genen Bedürfnissen stehender Ressourcen }\end{array}$ & \\
\hline $\begin{array}{l}\text { Aktivierung von Energie: Spürbare Zunahme } \\
\text { des Energieniveaus und der Zuversicht }\end{array}$ & \\
\hline $\begin{array}{l}\text { Entwickeln einer Handlungsoption: Benennen } \\
\text { von Handlungsschritten, gegebenenfalls auch } \\
\text { in Verbindung mit einer veränderten eigenen }\end{array}$ & $\begin{array}{l}\text { Anwendung der Veränderung auf das Um- } \\
\text { feld: Nach dem Erarbeiten einer Verände- } \\
\text { rung erfolgt eine Hinwendung zum Um- } \\
\text { feld, um das Erarbeitete dort zur Anwen- } \\
\text { dung zu bringen }\end{array}$ \\
\hline
\end{tabular}

Tabelle 10: Übersicht der Kernmerkmale der idealtypischen Episoden

Bei den kontrastierenden Episoden sind als übereinstimmende Merkmale erkennbar:

\begin{tabular}{|l|l|}
\hline Bezogen auf das Selbsterleben & Kontextbezogen \\
\hline $\begin{array}{l}\text { Emotionales Belastungserleben: Ein Erleben } \\
\text { von Belastetsein wird zum Ausdruck gebracht, } \\
\text { einhergehend mit dem Wunsch nach einer Ver- } \\
\text { änderung. }\end{array}$ & $\begin{array}{l}\text { Wahrnehmung problematischer Interak- } \\
\text { tion des Umfelds: Das eigene Erleben } \\
\text { wird negativ beeinflusst durch das Verhal- } \\
\text { ten des Umfelds. }\end{array}$ \\
\hline
\end{tabular}

Tabelle 11: Übersicht der übereinstimmenden Merkmale der kontrastierenden Episoden

Beide bei den kontrastierenden Episoden genannten Aspekte finden sich auch in den idealtypischen Prozessen, so dass davon auszugehen ist, dass sie nicht charakteristisch für die kontrastierenden Episoden sind, sondern zunächst eher allgemein im Kontext von Unklarheit auftauchen. Aus ihrem Vorhandensein allein kann insofern keine Prognose für den weiteren Verlauf der Verarbeitung abgeleitet werden.

Spezifische übereinstimmende Kernmerkmale weniger gelingender Prozesse ließen sich aus diesem Prozessschritt der Untersuchung nicht ableiten. Vor diesem Hintergrund wurde entschieden, zunächst die empirischen Modelle der idealtypischen Prozesse zu einem gemeinsamen empirischen Modell zu synthetisieren und die kontrastierenden Modelle anschließend zur Abgrenzung hinzuzuziehen. So wurden im nächsten Schritt die Diagramme der empirischen Modelle der idealtypischen Prozesse miteinander verglichen, und auch hier wurde nach übereinstimmenden Merkmalen gesucht. Der Vergleich wurde als Gegenüberstellung der Modelle in einem separaten Dokument vorgenommen, und Gemeinsamkeiten wurden dort handschriftlich markiert (sh. Anlage 61: Vergleich der empirischen Modelle). Daraus ließ sich unter Zuhilfenahme der oben beschriebenen übereinstimmenden Prozessmerkmale das folgende gemeinsame empirische Modell beschreiben. 


\subsubsection{Gemeinsames empirisches Modell}

Der Veränderungsprozess wird ausgelöst durch eine Situationsbeschreibung, die die Wahrnehmung problematischen Verhaltens im Umfeld beinhaltet, das in Bezug zur eigenen Person steht und das eigene Erleben negativ beeinflusst. Gleichzeitig wird in der Situation auf der affektiven und der kognitiven Ebene eine Spannung erlebt, die ausgelöst wird von einem Missverhältnis zwischen der eigenen Person und dem Umfeld oder von einem Missverhältnis unterschiedlicher Motivationsstränge in der eigenen Person.

In der folgenden Prozessphase kommt es zu einer Vertiefung der Situationsbeschreibung und zum Benennen des Unklaren im Sinn des Task Markers. Auf der einen Seite zeichnet sich diese Phase durch einen weiteren Kontextbezug in Sinn einer Bewertung bzw. Distanzierung vom Umfeld aus, und auf der anderen Seite erfolgt zu eine vertiefte Bezugnahme auf das innere Erleben, gekennzeichnet durch ein Experiencing der Stufe 4 oder höher (EXP $\geq 4)$. Die Bezugnahme auf das innere Erleben kann mit einem explizit beschriebenen unangenehmen bzw. auffälligen Körpererleben einhergehen, oder mit einem generellen Darstellen von emotionalem Belastetsein.

Es folgt eine Phase des Verweilens beim Selbsterleben. In dieser Phase findet keine oder nur eine begrenzte Bezugnahme zum Kontext statt, die Klientin beschäftigt sich primär mit inneren Vorgängen. Das Selbsterleben intensiviert sich, gekennzeichnet durch Experiencing der Stufe 5 oder höher (EXP $\geq 5$ ). In dieser Phase ist kein linearer Prozess erkennbar, sondern eher ein zirkuläres bzw. zwischen verschiedenen Facetten hin- und her-pendelndes Auseinandersetzen mit dem inneren Erleben. Dazu gehört, dass belastende Gefühle, belastendes Körpererleben oder auch die Problematik verdeutlichende Metaphern expliziert und weiter vertieft werden, so dass die Schwere der Belastung auch aktuell spürbar und in ihrer Bedeutung für die Situation erkennbar ist (felt sense). Dieses Spüren setzt einen weiteren Prozess in Gang, der verschiedene Elemente einer inneren Bewegung enthalten kann. Solche Elemente können sein: Reflektieren der eigenen Motivation einschließlich deren biographischer Einflüsse, Reflektieren der eigenen Bedürfnisse und ein Aktivieren diesbezüglicher Ressourcen, oder auch insgesamt eine Aktivierung von Energie. Zwischen diesen Elementen und dem Spüren der Schwere der Belastung findet ein mehrfacher Abgleich statt, der dann zunehmend zu einer Art des Verstehens des inneren Erlebens führt. Es kommt zu einer gewissen emotionalen Erleichterung (Felt Shift), und parallel zeichnet sich auf der Haltungs- oder Handlungsebene eine Lösung ab. Daraus wird eine Handlungsalternative entwickelt, die Task kann als bearbeitet betrachtet werden (Task Finish). Das Experiencingniveau nimmt in dieser Phase wieder ab, es liegt unter dem im Rahmen des Verweilens beim Selbsterleben erreichten Spitzenwert.

Wie das gemeinsame empirische Modell als Diagramm abgebildet werden kann, zeigt Abbildung 8 . 


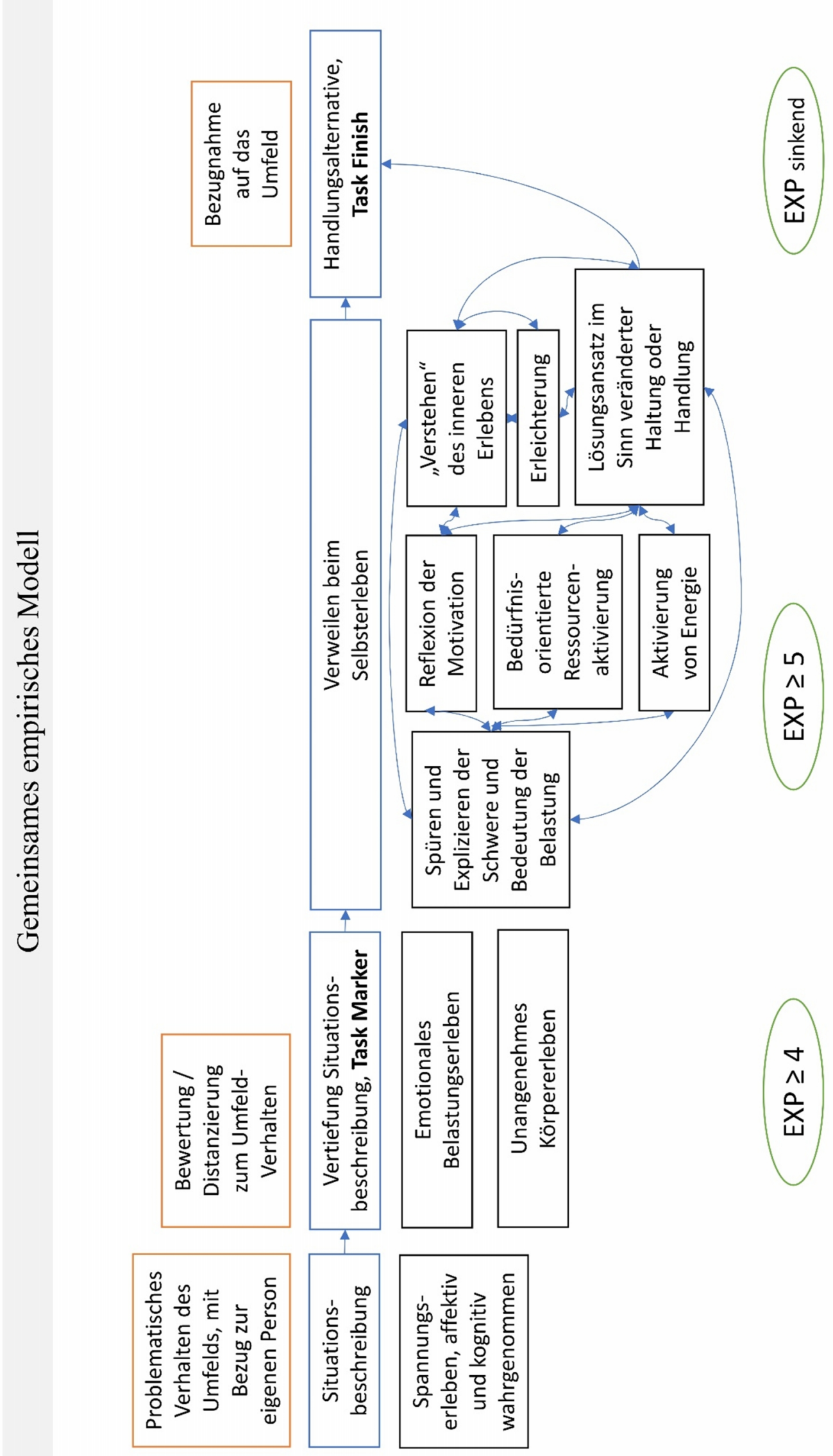

Abbildung 8: Gemeinsames empirisches Modell 


\subsubsection{Idealtypisches Modell und kontrastierende Modelle im Vergleich}

Um die idealtypischen Prozesse mit den kontrastierenden Prozessen zu vergleichen, interessierte zunächst, welche der gemeinsamen Kernmerkmale der idealtypischen Prozesse sich auch in den weniger gelingenden Prozessen wiederfinden. Dazu wurde eine Tabelle erstellt, in der dargestellt wurde, welche der Kernmerkmale idealtypischer Prozesse sich in den Higher-Level-Codierungen der kontrastierenden Prozesse (sh. Anlage 60: Empirische Analyse, Higher-Level-Codierungen, Abschnitt 1.2) wiederfinden:

\begin{tabular}{|c|c|c|c|}
\hline $\begin{array}{l}\text { Gemeinsame Kern- } \\
\text { merkmale idealtypi- } \\
\text { scher Prozesse, bezo- } \\
\text { gen auf... }\end{array}$ & \multicolumn{3}{|c|}{ Entsprechende Higher-Level-Codes in Episode... } \\
\hline ...das Selbsterleben & 2016_1119a_01\#1 & 2015_2007_02\#1 & 2017_0118_01\#2 \\
\hline Spannungserleben & & & $\begin{array}{l}\text { Entscheidungsdi- } \\
\text { lemma }\end{array}$ \\
\hline Emotionale Belastung & $\begin{array}{l}\text { Druck } \\
\text { Verhaltensänderung } \\
\text { bewirkt keine Ver- } \\
\text { besserung des Selbst- } \\
\text { erlebens } \\
\text { Hohe emotionale Be- } \\
\text { lastung } \\
\text { Emotionale Belas- } \\
\text { tung }\end{array}$ & $\begin{array}{l}\text { Emotionale Belas- } \\
\text { tung } \\
\text { Defiziterleben } \\
\text { Kraftlosigkeit }\end{array}$ & Unsicherheit \\
\hline Körpererleben & & & \\
\hline Metapher & & & \\
\hline $\begin{array}{l}\text { Reflexion der Motiva- } \\
\text { tion }\end{array}$ & $\begin{array}{l}\text { Veränderungs- } \\
\text { wunsch }\end{array}$ & $\begin{array}{l}\text { Veränderungs- } \\
\text { wunsch }\end{array}$ & \\
\hline $\begin{array}{l}\text { Bedürfnisorientierte } \\
\text { Ressourcenaktivie- } \\
\text { rung }\end{array}$ & & & $\begin{array}{l}\text { Eigene Position als } \\
\text { Referenzpunkt }\end{array}$ \\
\hline $\begin{array}{l}\text { Aktivierung von Ener- } \\
\text { gie }\end{array}$ & $\begin{array}{l}\text { Aktivieren von Ener- } \\
\text { gie }\end{array}$ & $\begin{array}{l}\text { Distanzierung von } \\
\text { emotionaler Belas- } \\
\text { tung } \\
\text { Erleichterung }\end{array}$ & \\
\hline
\end{tabular}




\begin{tabular}{|l|l|l|l|}
\hline $\begin{array}{l}\text { Entwickeln einer } \\
\text { Handlungsoption }\end{array}$ & Handlungsplan & Handlungsoption \\
\hline ..den Kontext & 2016_1119a_01\#1 & 2015_2007_02\#1 & 2017_0118_01\#2 \\
\hline $\begin{array}{l}\text { Wahrnehmung proble- } \\
\text { matischer Interaktion } \\
\text { des Umfelds }\end{array}$ & $\begin{array}{l}\text { Problematisches } \\
\text { Verhalten des Um- } \\
\text { felds } \\
\text { Umfeld als Ursache } \\
\text { für eigenes Unwohl- } \\
\text { befinden }\end{array}$ & $\begin{array}{l}\text { Belastendes Um- } \\
\text { feld } \\
\text { Problematisches } \\
\text { felds } \\
\text { Umfeld nicht als } \\
\text { Ressource }\end{array}$ & $\begin{array}{l}\text { Unklarheit bezüg- } \\
\text { lich der Positionie- } \\
\text { rung des Umfelds } \\
\text { zur eigenen Person }\end{array}$ \\
\hline $\begin{array}{l}\text { Distanzierung zum } \\
\text { Umfeld }\end{array}$ & $\begin{array}{l}\text { Anwenden der Verän- } \\
\text { derung auf das Umfeld }\end{array}$ & & \\
\hline
\end{tabular}

Tabelle 12: Kernmerkmale idealtypischer Prozesse und Codierungen der kontrastierenden Episoden im Vergleich

Darüber hinaus finden sich in den kontrastierenden Prozessen folgende Codierungen, die sich nicht den Kernmerkmalen gelingender Prozesse zuordnen lassen:

Makrokategorie Selbsterleben:

Episode 2016_1119a_01\#1:

- Die Kategorie „Mitgefühl mit dem Umfeld“ adressiert zwar die eigene Motivation, aber nicht im Sinn einer Reflexion, so dass sie nicht als Entsprechung für „Reflexion der Motivation gewertet wird.

- „Bedeutung des inneren Erlebens eröffnet sich nicht“.

- „Fehlende Handlungsfähigkeit“: weist darauf hin, dass das eigenständige Entwickeln einer Handlungsoption nicht gelingt. Im gleichen Kontext tauchen weitere Kategorien auf: „Agieren“, gleichzeitig „fehlende Problemlösung“, „Unzufriedenheit“, und nochmals „Fehlende Handlungsfähigkeit“.

- Am Ende der Episode: „Weitere, bisher nicht thematisierte Unklarheit““.

Episode 2015_2007_02\#1:

- „Erlebte Hilfsbedürftigkeit“: Könnte als emotionale Belastung gewertet werden, geht aber aufgrund der Auswirkung, der Hilfsbedürftigkeit, noch darüber hinaus.

Episode 2017_0118_01\#2:

- Keine zusätzlichen Codes, die sich nicht mit den Kernmerkmalen gelingender Prozesse decken würden. 
Makrokategorie Kontextbezug:

Episode 2016_1119a_01\#1:

- „Intensives Beschäftigen mit der Interaktion des Umfelds“: Geht über die Wahrnehmung problematischer Interaktion hinaus, entspricht aber auch nicht einer Distanzierung zum Umfeld und kann daher nicht den Kernmerkmalen idealtypischer Prozesse zugeordnet werden.

- „Verantwortung für das Umfeld“, „Handlungserwartung“, „Anforderungen des Umfelds“, „Identifikation mit dem Erleben des Umfelds“ (2x), „Rücksichtnahme auf die Befindlichkeit des Umfelds“: Dies sind weitere Aspekte, die im engen $\mathrm{Zu}$ sammenhang mit dem intensiven Beschäftigen des Umfelds stehen. Auch diese Kategorien gehen über die Wahrnehmung problematischer Interaktion hinaus und signalisieren, dass eine Distanzierung zum Umfeld nicht oder nur teilweise stattfindet.

Episode 2015_2007_02\#1:

- „Unklarheit bezüglich der Positionierung zum Umfeld“: unterscheidet sich von einer klaren Positionierung oder Distanzierung zum Umfeld und kann daher dort nicht zugeordnet werden.

Episode 2017_0118_01\#2:

- keine zusätzlichen Codes, die sich nicht mit den Kernmerkmalen gelingender Prozesse decken würden.

Im tabellarischen Vergleich der idealtypischen Kernmerkmale und der Codierungen der kontrastierenden Prozesse (Tabelle 12) fällt auf:

- Bei den kontrastierenden Episoden findet ebenso wie bei den idealtypischen Episoden eine Beschreibung von problematischem Interaktionsverhalten des Umfelds statt. Das gleichzeitige Spannungserleben wird bei den kontrastierenden Episoden allerdings nicht oder nur sehr rudimentär angesprochen.

- Eine Distanzierung zum Umfeld im Sinn einer Bewertung oder eigenen Positionierung findet bei den kontrastierenden Prozessen nicht statt.

- Die Kernmerkmale „Körpererleben“ und „Metapher“ sind bei den kontrastierenden Prozessen nicht zugeordnet. Dies geht einher damit, dass das ExperiencingNiveau bei den kontrastierenden Prozessen insgesamt niedriger ist als bei den idealtypischen Prozessen: Abgesehen von einem Spitzenwert EXP 5 in der ersten kontrastierenden Episode (2016_1119a_01\#1) bewegt es sich auch nach der Vertiefung der Situationsbeschreibung und dem Benennen der emotionalen Belastung zwischen EXP 2 und EXP 4 (sh. Abbildung 9: Experiencing-Stufen der untersuchten Episoden im Vergleich, als Prozessverlauf jeweils zu lesen von links nach rechts).

- Auch das Kernmerkmal „Reflexion der Motivation“ ist in den kontrastierenden 
Episoden unterrepräsentiert. Zwar wurde hier die Codierung „Veränderungswunsch“ zugeordnet, weil ein Veränderungswunsch auch Fragen der Motivation tangiert. Jedoch kann ein explizierter Veränderungswunsch nur als eine kleine, eher marginale Facette des Reflektierens der eigenen Handlungsmotivation und ihrer Einflussfaktoren gewertet werden. Ähnliches gilt für die beiden folgenden Kernmerkmale:

- „Bedürfnisorientierte Ressourcenaktivierung“: auch hier ist ein Code (,Eigene Position als Referenzpunkt") zugeordnet, der jedoch keinen Hinweis darauf gibt, dass die eigenen Bedürfnisse exploriert wurden, und der Code lässt auch offen, inwieweit letzten Endes eine Aktivierung der Ressourcen stattgefunden hat.

- „Aktivierung von Energie“: Explizit ist diese Codierung nur im Prozess repräsentiert, im zweiten Prozess gibt die Codierung indirekte Hinweise auf die Aktivierung von Energie, und im dritten Prozess findet sich keine entsprechende Zuordnung.

- Hinsichtlich des Kontextbezugs liegt bei den kontrastierenden Episoden zum Kernmerkmal „Anwendung der Veränderung auf das Umfeld“ keine Codierung vor.

Experiencing-Scale: EXP-Stufen im Vergleich

erste, zweite und dritte idealtypische Episode (2017_1805_01\#1,2016_1119a_01\#8, 2017_2119_03\#3)

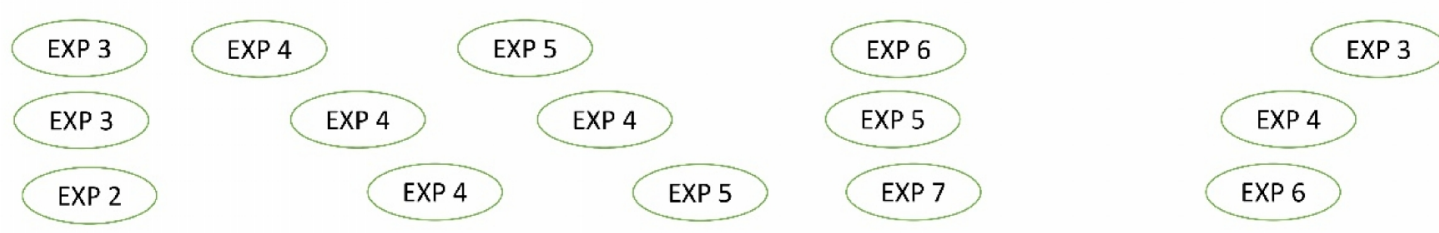

Gemeinsames empirisches Modell

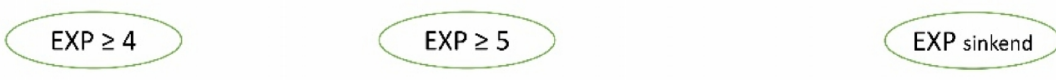

erste, zweite und dritte kontrastierende Episode (2016_1119a_01\#1,2015_2007_02\#1,2017_0118_01\#2)
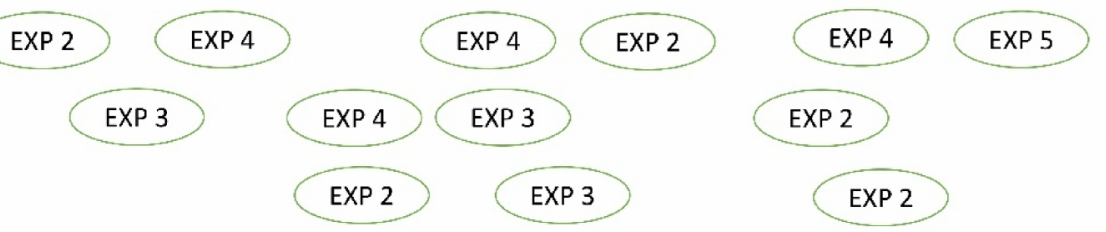

EXP 2

Abbildung 9: Experiencing-Stufen der untersuchten Episoden im Vergleich

In einem weiteren Vergleichsschritt wurde das Diagramm des gemeinsamen empirischen Modells idealtypischer Prozesse verglichen mit den Diagrammen der kontrastierenden Prozesse, und die auffallenden Unterschiede wurden in den Diagrammen zunächst handschriftlich markiert, um die Unterschiede der Prozesselemente herauszuarbeiten (sh. Anlage 61: Vergleich 
der empirischen Modelle). Die Unterschiede lassen sich wie folgt beschreiben.

Zum Modell der ersten kontrastierenden Episode (2016_1119a_01\#1):

- Hier wurde zum einen das Mitgefühl für das Umfeld in Verbindung mit der Verantwortung für das Umfeld markiert, und weiter die Identifikation mit dem Erleben des Umfelds und die Rücksichtnahme auf die Befindlichkeit des Umfelds in Unterscheidung zu dem Aspekt der Bewertung / Distanzierung zum Umfeldverhalten bei den gelingenden Prozessen. Dies legt nahe, dass die Bewertung / Distanzierung relevant sein könnte für das Gelingen des experienziellen Prozesses, während eine hohe Identifikation mit dem Umfeld diesen beeinträchtigen könnte.

- Auffallend ist auch, dass die Beschäftigung mit dem Umfeld in der kontrastierenden Episode das Selbsterleben überlagert, während im idealtypischen Prozess ein Verweilen beim Selbsterleben stattfindet, in dessen Verlauf eine Bezugnahme zum Umfeld nicht oder nur sehr eingeschränkt stattfindet.

- Inwieweit die beiden zuvor genannten Aspekte kausal zu sehen sind für die fehlende Problemlösung und das Nicht-Eintreten einer Erleichterung oder eines Lösungsansatzes, kann nicht beurteilt werden. Erkennbar ist aber, dass zwar ein Selbsterleben (EXP 4 und EXP 5) stattfindet, dieses aber nicht zu einer Lösung führt, sondern dass stattdessen im Umfeld weitere problematische Aspekte auftauchen, denen Aufmerksamkeit gewidmet wird. Im Vergleich mit den gelingenden Prozessen kann hier zwar auch von einem Selbsterleben gesprochen werden, nicht aber von einem Verweilen beim Selbsterleben.

Zum Modell der zweiten kontrastierenden Episode (2015_2007_02\#1):

- Hier wurde als Unterschied zum idealtypischen Modell markiert, dass im Rahmen der Situationsbeschreibung kein Kontextbezug stattfindet, also kein problematisches Verhalten des Umfelds geschildert wird. Stattdessen wird direkt die emotionale Belastung in Verbindung mit einem Veränderungswunsch, auch im Sinn eines Task Markers, thematisiert, während diese Prozessschritte im idealtypischen Modell erst im nächsten Prozessschritt, der Vertiefung der Situationsbeschreibung, enthalten sind. Es fehlt also ein gewisser Vorlauf für den weiteren Prozess im Sinn einer Bezugnahme auf den Kontext und einer Schilderung des Spannungserlebens.

- Es kommt schnell zu einem Experiencing der Stufe 4 (EXP 4), dieses nimmt dann aber wieder ab, und dort, wo im idealtypischen Modell ein Verweilen beim Selbsterleben (EXP $\geq 5$ ) beschrieben ist, findet im kontrastierenden Prozess kein Experiencing (EXP 2) statt, und auch kein zirkulärer Prozess im Sinn einer Verbindung zwischen dem Erleben und dem zu bearbeitenden Thema. In diesem Kontext bringt der Coach einen Vorschlag für eine Handlungsalternative ein, der von der Klientin aufgenommen wird. 
- Der Prozess endet mit dieser nicht aus dem Experiencing heraus entwickelten Handlungsalternative, gleichwohl bestätigt die Klientin, dass ihr diese auch Erleichterung verschafft.

Zum Modell der dritten kontrastierenden Episode (2017_0118_01\#2):

- Ähnlich wie die zweite kontrastierende Episode beginnt diese Episode nicht mit einem Vorlauf, bei dem problematisches Verhalten des Umfelds und eigenes Spannungserleben thematisiert wird. Stattdessen steht von Anfang an die Unklarheit bezüglich der Positionierung zum Umfeld im Vordergrund. Eine Distanzierung zum Umfeld gelingt nicht.

- Das Dilemma wird nicht als Spannungserleben mit affektiven Anteilen verarbeitet, sondern kognitiv-rationale Aspekte stehen im Vordergrund (EXP 2 und EXP 3). Aus diesen heraus erarbeitet die Klientin in kurzer Zeit eine Handlungsoption, die sie dann durch das Erstellen eines Handlungsplans konkretisiert.

- Es gibt im weiteren Verlauf keinen Hinweis darauf, dass das anfänglich beschriebene Dilemma nicht mehr als solches erlebt wird, das Selbsterleben wird nicht weiter thematisiert.

\subsubsection{Abgleich der theoriebasierten Codierungen mit dem empirischen Befund}

In einem weiteren Zwischenschritt wurden die drei idealtypischen Episoden hinsichtlich der aus dem theoretischen Modell abgeleiteten Kategorien untersucht. Ziel war hier, einen Überblick zu erhalten, welche der Kategorien sich in den Episoden wiederfinden. Dies erfolgte in Form einer einfachen Übersicht, indem die tabellarische Übersicht der Kategorien um die Vorkommen in den jeweiligen Episoden ergänzt wurde (1 steht für die erste idealtypische Episode, 2 steht für die zweite idealtypische Episode, 3 steht für die dritte idealtypische Episode; Mehrfachnennungen bedeuten, dass die jeweilige Kategorie in der Episode mehrfach zugeordnet wurde):

\begin{tabular}{|l|l|l|}
\hline $\begin{array}{l}\text { Hauptkategorie auf Basis } \\
\text { des theoretischen Modells }\end{array}$ & Unterkategorie & $\begin{array}{l}\text { Vorkommen } \\
\text { in den Episoden }\end{array}$ \\
\hline Thema / Situation & Situationsbeschreibung & $1,1,1,2,2,2,2,3,3$ \\
\hline & Irritation / Unbehagen & $1,2,2,2,3$ \\
\hline & Herausarbeiten der Unklarheit & \\
\hline & Fragestellung & 3 \\
\hline $\begin{array}{l}\text { Aktuelles Gefühl / Kör- } \\
\text { pererleben }\end{array}$ & $\begin{array}{l}\text { Weränderungswunsch } \\
\text { bens (Selbsterleben / referring } \\
\text { inward) }\end{array}$ & $1,2,3,3$ \\
\hline
\end{tabular}




\begin{tabular}{|c|c|c|}
\hline & $\begin{array}{l}\text { Wahrnehmung von Verände- } \\
\text { rung des inneren Erlebens }\end{array}$ & $\begin{array}{l}\text { 1, } 1 \text { (zu Beginn und zum } \\
\text { Ende der Task Bearbeitung), } \\
2,2\end{array}$ \\
\hline \multirow[t]{2}{*}{$\begin{array}{l}\text { Verbindung zwischen } \\
\text { Selbsterleben und Thema }\end{array}$} & $\begin{array}{l}\text { Bezugnahme auf das Selbster- } \\
\text { leben im Sinn einer Verbin- } \\
\text { dung zwischen Situation und } \\
\text { innerem Erleben (Felt Refe- } \\
\text { rent, Felt Sense) }\end{array}$ & $1,2,2,2,3,3,3,3$ \\
\hline & Verweilen beim Selbsterleben & $1,2,2,2,3$ \\
\hline \multirow{3}{*}{$\begin{array}{l}\text { Themenbezogene Bedeu- } \\
\text { tung }\end{array}$} & Symbolisierung anwenden & \\
\hline & Explizieren von Bedeutung & $1,3,3$ \\
\hline & $\begin{array}{l}\text { Deutungsalternativen / Neu- } \\
\text { bewertung des Themas }\end{array}$ & $1,3,3$ \\
\hline \multirow[t]{3}{*}{ Veränderungsmoment } & Felt Shift / Erleichterung & $1,2,2,3$ \\
\hline & Erlebte Stimmigkeit & 2,3 \\
\hline & Erleben von Souveränität & 1,1 \\
\hline \multirow[t]{2}{*}{$\begin{array}{l}\text { Handlung / Handlungs- } \\
\text { plan }\end{array}$} & $\begin{array}{l}\text { Entwickeln von Handlungsal- } \\
\text { ternativen }\end{array}$ & $1,2,2,2,3,3$ \\
\hline & Kongruenzerleben & \\
\hline
\end{tabular}

Tabelle 13: Kategorien des theoretischen Modells und ihre Vorkommen in den idealtypischen Episoden

Daraus lässt sich schließen, dass folgende Kategorien des theoretischen Modells in den untersuchten Episoden keine oder allenfalls eine untergeordnete Rolle spielen:

- Herausarbeiten der Unklarheit (Hauptkategorie Thema / Situation)

- Fragestellung (Hauptkategorie Thema / Situation)

- Anwenden der Symbolisierung

- Kongruenzerleben.

Dem steht gegenüber, dass die Situationsbeschreibung und die Irritation bzw. das Unbehagen vergleichsweise häufig codiert wurden. Daraus lässt sich ableiten, dass die theoriebasiert entwickelte Hauptkategorie Thema / Situation angepasst werden sollte an den empirischen Befund: Das Herausarbeiten der Unklarheit und die Fragestellung rücken in den Hintergrund, dafür kommen in dieser Hauptkategorie die empirisch entwickelten Kernelemente Spannungserleben, Emotionale Belastung, Wahrnehmung problematischer Interaktion des Umfelds und Distanzierung zum Umfeld stärker ins Blickfeld. 
Hinsichtlich der Hauptkategorie Themenbezogene Bedeutung, zu der die Unterkategorie Anwenden der Symbolisierung gehört, fällt zunächst auf, dass diese insgesamt eher wenig repräsentiert ist in den untersuchten Coachinggesprächen. Gleichzeitig ist erkennbar, dass die Verbindung zwischen Selbsterleben und Thema vergleichsweise häufig codiert wurde, und zwar sowohl im Blick auf die Bezugnahme zwischen innerem Erleben und Situation als auch im Blick auf das Verweilen beim Selbsterleben. Gerade die Episode 2, die in der Hauptkategorie Themenbezogene Bedeutung nicht codiert ist, ist hier mit einigen Codierungen repräsentiert, und dann auch wieder in der Hauptkategorie Veränderungsmoment. Auch bei den Episoden 1 und 3 ist die Verbindung zwischen Selbsterleben und Thema und daran anschließend ein Veränderungsmoment kategorisiert, so dass davon ausgegangen werden muss, dass dem inneren Erleben zwar eine Bedeutung gegeben wurde, dass dieses aber auf eine Art geschah, die sich mit den Kategorien des theoretischen Modells nicht ideal erfassen lassen. Auffallend ist dabei, dass das Verweilen beim Selbsterleben in den untersuchten Episoden nicht in erster Linie als Verweilen beim Körpererleben und dessen gefühlter Bedeutung stattfindet. Das Verweilen beim Selbsterleben erscheint dort weit gefasst, indem zum Beispiel eine Metapher entsteht, die zwar nicht als „Griff“ im Sinn des Focusing verstanden werden kann, wohl aber als Explikation des Selbsterlebens unter Bezugnahme auf die Situation. Auch das Reflektieren der eigenen Motivation einschließlich biographischer Einflüsse oder das Entdecken eigener Bedürfnisse und Ressourcen kann hier als Teil des Verweilens beim Selbsterleben verstanden werden, der dazu beiträgt, eine themenbezogene Bedeutung des aktuellen Selbsterlebens bzw. Körpererlebens zu entwickeln.

Beim Betrachten der Unterkategorie Kongruenzerleben fällt auf, dass diese im theoretischen Aufgabenmodell nicht eindeutig von der Kategorie Erlebte Stimmigkeit abgegrenzt wurde. Die Abgrenzung der beiden Kategorien besteht im Wesentlichen darin, dass die erlebte Stimmigkeit der Hauptkategorie Veränderungsmoment zugeordnet ist, während das Kongruenzerleben sich bei der Hauptkategorie Handlung / Handlungsplan wiederfindet, so dass der Eindruck entsteht, dass im Zuge der Erarbeitung eines Handlungsplans nochmals eine Bezugnahme auf das Selbsterleben stattfindet, die die Übereinstimmung des Selbsterlebens bzw. der körperlich gefühlten Bedeutung mit dem nun entwickelten Handlungsplan bestätigt. Der empirische Befund legt aber eher nahe, dass die Bezugnahme auf das Selbsterleben zu diesem Zeitpunkt nicht mehr so ausgeprägt stattfindet, sondern dass hier stärker der Kontextbezug der Veränderung in den Mittelpunkt rückt.

Diese Aspekte werden im nächsten Schritt, bei dem das theoretische Modell und das gemeinsame empirische Modell synthetisiert werden sollen, zu berücksichtigen sein.

\subsubsection{Synthetisiertes theoretisch-empirisches Modell}

Das theoretische Modell geht von einer am Anfang des Prozesses stehenden themenbezogenen Unklarheit bzw. Irritation aus, in deren Folge sich ein aktuelles Gefühl oder Körpererleben einstellt. Diese Ausgangsbasis zum Einstieg in einen Veränderungsprozess wird vom empirischen Modell nicht widerlegt, aber doch in gewisser Weise differenziert. Hier kann eine 
Situationsbeschreibung als Prozessauslöser gesehen werden, die zum einen ein problematisches Verhalten des Umfelds in Bezug zur eigenen Person beinhaltet, und zum anderen ein Spannungserleben, das als unangenehm wahrgenommen wird. Die Differenzierung zum theoretischen Modell besteht also in der Anfangsphase darin, dass eine Situation beschrieben wird, die zwar abstrahierend als Irritation oder Unklarheit betrachtet werden kann, die sich im Moment des Geschehens aber als Person-Umfeld-Problem bei gleichzeitig affektiv-kognitivem Spannungserleben darstellt.

In einem nächsten Abschnitt des Veränderungsprozesses kommt es zu einer Vertiefung der Situationsbeschreibung. Vertiefung bedeutet in diesem Fall, dass es um mehr geht als um eine Wiederholung der Situationsbeschreibung, auch um mehr als das Benennen von weiteren Details, sondern dass sowohl bezüglich der Kontextwahrnehmung als auch bezüglich des Selbsterlebens ein vertiefender und gleichzeitig vorantragender Prozessschritt erfolgt. Hinsichtlich des Person-Umfeld-Problems findet eine gewisse Positionierung statt, und zwar in Form einer Distanzierung zum Umfeld. Diese Distanzierung kann unterschiedlich ausgeprägt sein, sie kann beispielsweise in Form einer Bewertung stattfinden oder sich auf einzelne Aspekte des Umfeldverhaltens beziehen.

Die Veränderung im Selbsterleben besteht in einer Art „,referring inward“, in Form einer Konzentration auf einen inneren Bezugsrahmen, in dem innere Erlebnisse weiterverarbeitet werden. Die im theoretischen Modell dargestellte Abfolge von „Aktuelles Gefühl oder Körpererleben“ und „Wahrnehmen des Gefühls bzw. Körpererlebens“ zeigt sich im empirischen Befund nicht als zeitlich versetzt stattfindend, sondern eher als ein gleichzeitiges Vorhandensein des Körpererlebens und der Wahrnehmung des Gefühls, als ein inneres „Etwas“, dem zunehmend Aufmerksamkeit gewidmet wird. Auf der Experiencing-Skala zeigt sich diese Zuwendung zum Selbsterleben in Form einer Experiencing-Zunahme (EXP $\geq 4)$. Beobachtbar wird sie dadurch, dass ein emotionales Belastungserleben beschrieben wird, das aktuell für die Klientin spürbar ist und sich im Verbalisieren von Gefühlen oder eines (unangenehmen) Körpererlebens ausdrückt. Für die Klientin geht das einher mit einer Aufmerksamkeitsverlagerung hin zum aktuellen Erleben, einer gewissen Irritation oder einem internalen Spannungserleben, und mit einem explizit oder implizit ausgedrückten Veränderungswunsch.

Die weitere im theoretischen Modell beschriebene Phase beinhaltet mehrere Schleifen, in denen eine Verbindung zwischen Thema und innerem Erleben bearbeitet und dem inneren Erleben eine themenbezogene Bedeutung gegeben wird. Im Wesentlichen bestätigen die empirischen Modelle diese Phase. Die Bedeutung des inneren Erlebens wird aber weniger fokussiert erarbeitet als das theoretische Modell dies vermuten lässt. Vielmehr scheint aufgrund der Vertiefung der Situationsbeschreibung und dem damit verbundenen Selbsterleben ein komplexer Explorationsprozess in Gang zu kommen, bei dem die Klientin einerseits die Schwere der Belastung spürt und den eigenen Bedürfnissen nachgeht, und andererseits eigene Ressourcen und Energie aktiviert. Es scheint also nicht nur eine Pendelbewegung zwischen Emotion 
bzw. Körpererleben und themenbezogener Bedeutung zu geben, sondern vor allem eine Pendelbewegung zwischen dem, was als schwierig und belastend erlebt wird, und dem, was Ressourcen und Energie freisetzt. Eine wichtige Rolle scheint dabei das „Verstehen“ des inneren Erlebens zu spielen, das zu einer veränderten Haltung, Erleichterung oder Klarheit führt oder mit dieser einher geht. Es ist eine Zunahme des Experiencing bis hin zu Stufe 5 oder höher $(\mathrm{EXP} \geq 5)$ zu beobachten, so dass die Klientin durch die Arbeit am bzw. mit dem unmittelbaren Erleben Gefühlsänderungen oder eine Verlagerung gefühlter Bedeutungen erfährt und diese zur Grundlage weiterer Überlegungen machen kann.

Um zu verdeutlichen, dass das Selbsterleben in dieser Phase im Zentrum des Geschehens steht, soll diese Phase im empirisch-theoretischen Modell analog zum gemeinsamen empirischen Modell als „Verweilen beim Selbsterleben“ gekennzeichnet werden. Weiter ist in den empirischen Modellen das Spüren der Schwere der emotionalen Belastung als ein erster wichtiger Aspekt des Verweilens beim Selbsterleben dargestellt. Vor dem Hintergrund des theoretischen Modells und der weit gefassten Definition des Task Markers ist allerdings zu hinterfragen, ob diese Beschreibung nicht zu eng gefasst ist. Als übergeordnete Kategorie könnte auch allgemeiner ein weiteres Zuwenden zum Selbsterleben beschrieben werden, in dem Sinn, dass dieses zunehmende Bedeutsamkeit erhält, die zu einer weiteren Selbstexploration führt. Es entsteht also eine Art innerer Bezugspunkt, der dann die weiteren Prozesse wie Reflexion der Motivation, Reflexion der Bedürfnisse, Aktivierung von Ressourcen und Aktivierung von Energie in Gang setzt und zu einem besseren Verstehen des eigenen gegenwärtigen Erlebens führt. Es kommt zu einer Veränderung hinsichtlich der Bewertung oder Deutung der Situation, die auch mit einer gewissen Erleichterung oder inneren Klarheit einher geht. An dieser Stelle deckt sich die empirische Analyse mit dem theoretischen Modell.

Die veränderte Bewertung oder Deutung führt zu einem Lösungsansatz, der entweder in einer veränderten Haltung besteht, die dann auch zu einem veränderten Umgang mit der Situation führt, oder zu einer Handlung oder einem Handlungsplan. Dessen Auswirkungen auf das Umfeld werden noch abschließend thematisiert. Das Experiencing-Niveau sinkt zu diesem Zeitpunkt wieder (EXP sinkend), der Blick ist in zunehmendem Maß auch wieder auf das Umfeld gerichtet und nicht primär auf das Selbsterleben.

Das aus diesen Beschreibungen abgeleitete Prozessdiagramm ist in Abbildung 10 dargestellt. 


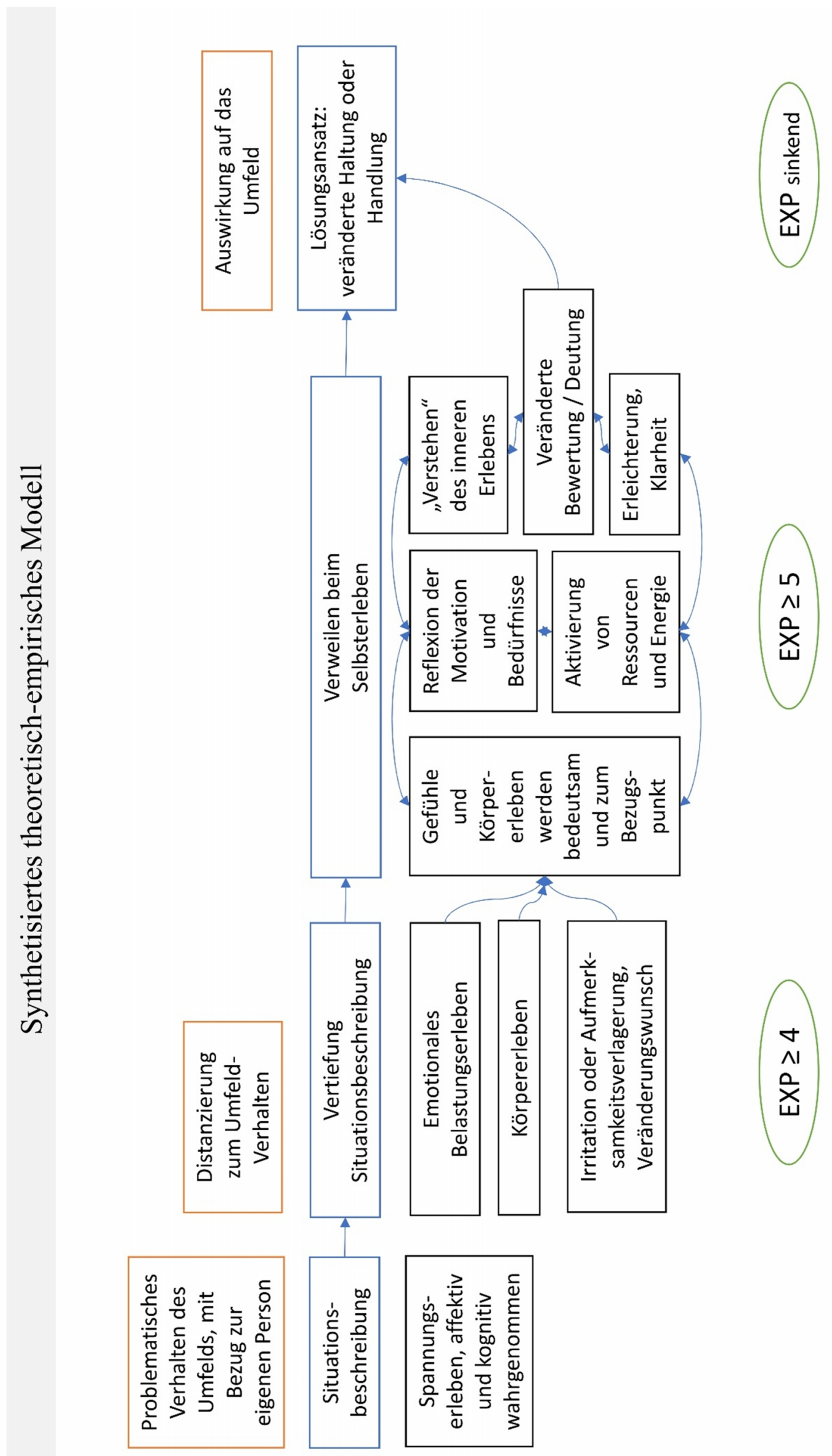

Abbildung 10: Synthetisiertes theoretisch-empirisches Modell 


\subsubsection{Theoretische Rückbindung: Der Felt Sense im Coaching}

Das aus den theoretischen Überlegungen und den empirischen Beobachtungen synthetisierte Modell kann als Weiterentwicklung bzw. Differenzierung des theoretischen Modells betrachtet werden, das die generelle Sicht auf Veränderung im Kontext von Experiencing bestätigt und gleichzeitig coachingspezifische Besonderheiten aufzeigt.

Das Modell beschreibt intrapersonale Veränderung als einen Prozess, bei dem sowohl Aspekte bewusster Kognition als auch affektive, emotionale und motivationale Elemente unter Einbeziehung körperbezogener Aspekte verarbeitet werden. Der Veränderungsprozess ist getragen davon, dass aus der Auseinandersetzung mit dem eigenen Erleben im Kontext des Umfelds bzw. der als problematisch beschriebenen Situation nicht nur ein Veränderungswunsch entsteht oder ein bereits zuvor bestehender Veränderungswunsch expliziert wird, sondern dass auch eigene Ressourcen und Energien aktiviert werden, die dann zu einer veränderten Haltung oder Handlung im Sinn einer persönlichen Weiterentwicklung führen. Aus personzentrierter bzw. experienzieller Sicht bestätigt sich damit zunächst die Sicht auf den Menschen als selbstbestimmtes und zur Weiterentwicklung hin orientiertes Wesen.

Festzustellen ist ebenso, dass hinsichtlich der experienziellen Theorien eine eindeutige $\mathrm{Zu}$ ordnung des Modells zum experienziellen Ansatz nach Gendlin (vgl. Kapitel 3.2), zur experienziellen Theorie nach Sachse (vgl. Kapitel 3.5) oder zur prozess-experienziellen emotionsfokussierten Theorie nach Greenberg (vgl. Kapitel 4.3.5) nicht möglich ist. Wenn die Klientin im ersten idealtypischen Beispiel feststellt, ,ich merk gerade, wie das in mir pocht“, dieses Pochen später als Wut identifiziert und mit einer problematischen Situation in Verbindung bringt, zeigt sich der Körper als Träger impliziten Wissens und komplexen Fühlens im Sinn der Konzeption Gendlins. Wenn die Klientin im dritten idealtypischen Prozess eine Sensibilität in einer bestimmten Region ihres Oberkörpers als Indikator für die Bewertung einer Situation beschreibt, wird nachvollziehbar, wie Sachse körperliche Reaktionen als Informationsträger affektiver Bedeutungen konzipiert. Und wenn in der zweiten idealtypischen Episode im Zusammenhang mit dem Erleben von Fremdbestimmung das Körperempfinden „Gelähmtsein“ und die Metapher „Eingesperrtsein“ beschrieben werden, lässt sich das nach Greenberg als ein körperlich und visuell repräsentiertes internes Verarbeitungsschema der Klientin einordnen.

Auch wenn eine eindeutige Zuordnung nicht möglich ist und es auch nicht das Ansinnen dieser Arbeit ist, die existierenden Konzeptionen zum Experiencing zu verifizieren oder zu falsifizieren, soll doch festgehalten werden, dass die Theorie-Konzeption Gendlins, in der der körperlich gespürte Felt Sense zum grundlegenden Prozess wird, sich nicht eindeutig bestätigen lässt. Einerseits legen die Ergebnisse der Task Analyse nahe, dass körperliche Reaktionen als Informationsträger affektiver Bedeutungen genutzt werden können. Andererseits lässt sich der körperlich gespürte Felt Sense nicht generell als Ausgangspunkt des Veränderungsprozesses bestätigen, vielmehr gelangen die Klienten oft erst im Rahmen des dialektischen Hin- 
und Her-Bewegens zwischen affektiven und kognitiven Verarbeitungsanteilen zu einer körperlich gespürten Komponente, die dann in der Regel für die Erarbeitung einer Lösung genutzt werden kann.

Hier unterscheidet sich der empirische Befund von einer rein focusingorientierten Sicht. Gemäß der Konzeption Gendlins zum Felt Sense nutzt die Focusing-Methode das Körpererleben gezielt als Ausgangspunkt für einen Veränderungs- oder Lösungsprozess. Die Befunde der Coachingprozesse legen nahe, dass zumindest dann, wenn ein Veränderungsprozess ohne explizite Focusing-Anleitung und trotzdem im Kontext von Experiencing stattfindet, eher eine Parallele zur Konzeption Sachses zu sehen ist, bei der im Rahmen affektiver Verarbeitung körperliche Reaktionen auftreten, die aber mehr sind als rein körperliche Reaktionen, sondern affektive Bedeutungen beinhalten, die der Klientin bis dahin noch nicht zugänglich waren und die nun expliziert werden können. Körpererleben muss also nicht Ausgangspunkt für einen Veränderungsprozess sein, sondern kann auch dann Teil des Lösungswegs sein, wenn es zum Beginn des Veränderungsprozesses noch nicht wahrgenommen wurde.

Im Vergleich des empirisch-theoretischen Modell mit der prozess-experienziellen emotionsfokusssierten Theorie Greenbergs lässt sich feststellen, dass die im Modell enthaltenen Aspekte des körperlichen Spürens in Greenbergs Konzeption zwar nicht ausgeschlossen werden, dort aber eine eher untergeordnete Rolle spielen. Im Kontext von Veränderung und Experiencing scheinen diese Aspekte aber doch eine wichtige Informationsquelle bzw. Ressource zu sein, die mehr als eine nur untergeordnete Rolle spielt.

Ein weiterer Aspekt, bei dem eine klare Zuordnung bzw. Stringenz schwerfiel, war die Differenzierung zwischen den Begriffen Gefühl, Emotion und Affekt. Auch die Termini Körpererleben, Körperspüren und Körpergefühl waren im Rahmen der Analyse nicht klar voneinander abzugrenzen. Dieser Erfahrung bei der Modellentwicklung ist sicher im Rahmen der Methodenkritik Rechnung zu tragen. Für die theoretische Rückbindung des Modells kann sie aber auch als Hinweis auf die Komplexität des Felt Sense aufgefasst werden, der im Modell auch als eine Art Metakategorie verstanden werden kann, die aus einem Zusammenwirken sowohl affektiver als auch kognitiver Verarbeitung entsteht und bei der Gefühle eine eigene Unterkategorie darstellen, die Aspekte affektiver, emotionaler und körperlicher Reaktionen einschließt (sh. auch Sachse \& Langens, 2014, S. 34).

Auch im Diagramm des theoretisch-empirischen Modells zeichnet sich eine gewisse Komplexität innerer Verarbeitung ab, bei der sowohl Kognitionen als auch Affekte und Emotionen eine Rolle spielen. Der Unterscheidung zwischen emotionaler und affektiver Verarbeitung (Sachse \& Langens, 2014, Benecke, 2014) folgend, wie sie in Kapitel 3.1 vorgestellt wurde, lassen sich jedoch zwei aufeinander aufbauende Phasen im Prozess des Selbsterlebens beschreiben: zum einen lässt sich die eher explizit und kognitiv orientierte emotionale Verarbeitung beobachten, insbesondere auch bei der Reflexion von Motivations- und Bedürfnisfragen, und auch bei der Aktivierung von Ressourcen und Handlungsbereitschaften. Davor aber 
findet sich eine Phase der affektiven Verarbeitung, die eher implizit und perzeptuell-sensumotorisch orientiert ist. Im empirisch-theoretischen Modell findet die affektive Verarbeitung parallel zur Vertiefung der Situationsbeschreibung statt. Hier wendet sich die Klientin mehr dem inneren Erleben zu, bewegt sich aber noch in gewisser Weise im Bereich des Spürens, dass da „Etwas“ ist, was ihre Aufmerksamkeit verdient. Dieses „Etwas“ ist genährt von zurückliegenden Erfahrungen und Erlebensweisen, die im Zusammenhang mit der Situation bzw. dem Umfeld stehen, die aber im Moment nicht nur als in der Vergangenheit liegend wahrgenommen werden, sondern auch aktuelle Gefühle und ,eine mehr oder weniger diffuse körperliche Reaktion“ (Sachse \& Langens, 2014, S. 34) auslösen und in gewisser Weise im Erleben präsent sind. Insofern findet nicht nur ein Reflektieren einer zurückliegenden Erfahrung statt, sondern auch eine Auseinandersetzung mit aktuellem Erleben. Dem entspricht auch die Experiencing Stufe 4 oder höher (EXP $\geq 4$ ), in der die Klientin nach Dahlhoff \& Bommert (1978, S. 73) Ereignisse, Situationen und das eigene Fühlen nicht mehr von außen betrachtet, sondern eine innerlich gefühlte Bedeutung entwickelt, auf die sie in ihren Mitteilungen Bezug nimmt. Im Rahmen des Verweilens bei diesem Erleben kommt es dann zu emotionalen Verarbeitungsprozessen, innerhalb derer auch lebens- oder berufsbiographische „Exkurse“ oder das Eruieren eigener Bedürfnisse und Ressourcen stattfinden können, die in die Zukunft gerichtete Handlungsimpulse und Wunschvorstellungen der Person aktivieren bzw. zu einer physiologischen Aktivierung und Veränderung des mentalen Zustands führen.

Beim Vergleich der Kategorien wurde bereits berichtet, dass die Hauptkategorie Themenbezogene Bedeutung, zu der die Unterkategorie Anwenden der Symbolisierung gehört, in den untersuchten Coachinggesprächen auffallend wenig repräsentiert ist. Dies überrascht zunächst, denn das Versehen einer gefühlten Bedeutung mit Wortsymbolen wird im Focusing als wichtiger Schritt betrachtet, ohne den die gefühlte Bedeutung unvollständig bleibt (vgl. Kapitel 3.2). Gleichzeitig ist die Verbindung zwischen Selbsterleben und Thema vergleichsweise häufig codiert, und zwar sowohl im Blick auf die Bezugnahme zwischen dem inneren Erleben und der Situation als auch im Blick auf das Verweilen beim Selbsterleben. Gerade die Episode 2, die in der Hauptkategorie Themenbezogene Bedeutung nicht codiert ist, ist hier mit einigen Codierungen repräsentiert, und dann auch wieder in der Hauptkategorie Veränderungsmoment. Auch bei den Episoden 1 und 3 ist die Verbindung zwischen Selbsterleben und Thema und daran anschließend ein Veränderungsmoment kategorisiert, so dass davon ausgegangen werden muss, dass dem inneren Erleben auch in den untersuchten Episoden eine Bedeutung gegeben wurde, dass dieses aber auf eine Art geschah, die sich von dem Prozess, wie er im Kontext des Focusing beschrieben wird (vgl. Kapitel 3.2) unterscheidet. Wenn der Aspekt der Symbolisierung bei Gendlin stark damit verbunden ist, ein Wort, eine Redewendung oder ein Bild, zu finden, das die Qualität des Felt Sense möglichst genau beschreibt, liegt nahe, dass die Symbolisierung zu einem Zeitpunkt entsteht, zu dem die ganze Aufmerksamkeit der Klientin dem Körpererleben und dem Explorieren der gefühlten Bedeutung gilt. Diese Aufmerksamkeits-Fokussierung ist im experientiellen Vorgehen erwünscht und wird 
durch die Focusing-Methode auch gezielt unterstützt. In Unterscheidung dazu findet bei den untersuchten Episoden auch ein Verweilen beim Selbsterleben statt, allerdings nicht primär im Sinn eines Verweilens beim Körpererleben und dessen gefühlter Bedeutung. Das Verweilen beim Selbsterleben erscheint dort weiter gefasst, indem zum Beispiel eine Metapher entsteht, die zwar nicht als „Griff“ im Sinn des Focusing verstanden werden kann, wohl aber als Explikation des Selbsterlebens unter Bezugnahme auf die Situation. Auch das Reflektieren der eigenen Motivation einschließlich biographischer Einflüsse oder das Entdecken eigener Bedürfnisse und Ressourcen kann hier als Teil des Verweilens beim Selbsterleben verstanden werden, der dazu beiträgt, eine themenbezogene Bedeutung des aktuellen Selbsterlebens einschließlich des Körpererlebens zu entwickeln.

Das legt nahe, dass der Begriff der Symbolisierung, wie er im Focusing-Konzept beschrieben ist, für das Coaching differenzierter eingeordnet werden muss. Wenn im Focusing-Konzept im Zusammenhang mit dem Felt Sense von der Symbolisierung erlebter Bedeutung am Rand des Gewahrwerdens (Sachse et al, 1992, S. 2) die Rede ist und diese als wesentliches Element im vorwärtstragenden Prozess persönlicher Veränderung bezeichnet wird (Feuerstein \& Müller, 1998, S. 42), ist dies eng damit verbunden, dass ein inneres Bezugsobjekt zum Bedeutungsträger und durch das Versehen mit Wortsymbolen beschreibbar wird. Im Coaching, wenn es nicht dezidiert nach der Focusing-Methode stattfindet, werden solche Wortsymbole nicht gezielt formuliert. Das Modell zeigt aber in zwei Facetten auf, wie die Symbolisierung erlebter Bedeutung im Coaching von statten gehen kann:

1. Zum einen wird das Fühlen oder Körpererleben zum Bezugspunkt, und es entsteht eine gewisse Ahnung der Bedeutsamkeit und Bedeutung dieses Fühlens oder Körpererlebens. Dann folgt eine Phase, in der das Experiencing sich weiter intensiviert bis hin zu Stufe EXP 5 oder höher (EXP $\geq 5$ ). Das bedeutet, dass die Klientin sich weiter auf bisher vage, nicht ausformulierbare aber bedeutsame Aspekte des unmittelbaren Erlebens bezieht und diese allmählich differenzieren kann. Daraus entsteht das, was im Diagramm als „Verstehen des inneren Erlebens“ bezeichnet ist. Symbolisierung geschieht hier also weniger im Finden des Wortsymbols als mehr in Form eines veränderten „Verstehens des inneren Erlebens“, und mit Blick auf die theoretischen Konzepte wäre das „Verstehen des inneren Erlebens“ möglicherweise leichter als Deutung im psychoanalytischen Sinn (vergleiche Kapitel 4.3.3) einzuordnen denn als Symbolisierung im experienziellen Sinn. Aus experienzieller Sicht kann der Vorgang aber ebenso als kognitive Repräsentation eines affektiven Schemas aufgefasst werden (Sachse \& Fasbender, 2011, S. 135), und der Felt Shift zeigt sich dann als unmittelbares Verstehen, das zum einen eine Aktivierung der Affekte mit sich bringt, und zum anderen zu einer veränderten Bewertung / Deutung führt. Aktivierung der Affekte bedeutet hier, dass nicht in jedem Fall eine Erleichterung gefühlt werden muss, es können auch, wie im Modell 
zur dritten idealtypischen Episode, noch weitere Affekte entstehen oder Teile „zurückbleiben“, die dann weiter bearbeitet werden können

2. Zum anderen ist im empirischen Modell die Pendelbewegung zwischen belastenden Elementen und Energie und Ressourcen freisetzenden Elementen des inneren Erlebens beschrieben. Diese Pendelbewegung ermöglicht es der Klientin, unterschiedliche Facetten des Selbsterlebens zu erfassen und so das anfängliche, auch körperlich spürbare Belastungserleben insofern zu transformieren, als dass sie motivationale und bedürfnisorientierte Informationen aus dem Körpererleben ableitet, die dazu beitragen, das Belastungserleben zu überwinden.

Die im empirisch-theoretischen Modell beschriebene Zirkularität des Selbsterlebens lässt sich vor dem Hintergrund von Gendlins Prozessmodell (vgl. Kapitel 3.5) plausibel nachvollziehen: Sie ist nicht im Sinn von weitgehend unveränderten Wiederholungsschleifen zu verstehen, sondern mehr im Sinn eines voran tragenden Prozesses in dessen Verlauf sich sowohl explizierte Bedeutungsinhalte als auch das innere Bezugsobjekt selbst verändern, so dass in der Gegenwart etwas Neues entsteht, das auch einen veränderten Blick auf die Vergangenheit ermöglicht (Gendlin, 1996, S. 31).

Hinsichtlich des Experiencing ist aus therapeutischen Prozessen bekannt, dass ein hohes Experiencingniveau (EXP 4 bis EXP 7) regelmäßig einhergeht mit einem signifikant positiven Therapie-Outcome, und dass bei erfolgreichen Therapieverläufen eine Zunahme des Experiencingniveaus bei der Bearbeitung problematischer Kernpunkte zu verzeichnen ist (Watson et al, 2010, S. 139; sh. Kapitel 3.4). Auch wenn sich aus der vorliegenden qualitativen Task Analyse keine statistisch valide Aussage ableiten lässt, kann hierzu festgehalten werden, dass die untersuchten Coachingprozesse dieser Aussage zum Verlauf des Experiencing nicht widersprechen: in allen drei Beispielen idealtypischer Prozesse nimmt das Experiencing im Verlauf des Verweilens beim Selbsterleben zu, bis hin zu den Stufen EXP 5, EXP 6, EXP 7. Im Gegensatz dazu ist das Experiencing bei den kontrastierenden Episoden niedriger und auch teilweise abnehmend (sh. Abbildung 9 in Kapitel 6.8.4: Experiencing-Stufen der untersuchten Episoden im Vergleich), und auch im Rahmen des Sampling-Prozesses fanden sich kaum Prozesse, bei denen in Folge des Task Markers zwar ein hohes Experiencing beschrieben werden konnte, sich aber kein Erfolg beim Task Finish einstellte (sh. Anlage 2: Forschungstagebuch). 


\section{Implikationen für das Coaching}

In der vorliegenden Untersuchung ging es darum, die Rolle des Felt Sense im Coaching näher zu erforschen und Erkenntnisse darüber zu gewinnen, was Coaching-Klienten über ihre inneren Prozesse im Coaching berichten und welche Besonderheiten intrapersonaler Veränderung sich für das Coaching beschreiben lassen. Ziel war, daraus Erkenntnisse über Experiencing und Veränderung im Rahmen von Coaching abzuleiten und diese für die Coachingpraxis zur Verfügung zu stellen. So sollen hier Rückschlüsse für die Coachingpraxis, die sich aufgrund des erarbeiteten empirisch-theoretischen Modells und der Vorüberlegungen dazu ergeben, dargestellt werden.

\subsection{Selbsterleben als Ressource}

„Aber es ist / es hat was ausgelöst, wo ich mir das gedacht habe, das geht überhaupt nicht und dann kann ich 10 mal wohin gehen und schauen wie ich mit dem Ganzen zurechtkomme, es passt nicht. Anscheinend hat er es bei allen gemacht, nur die machen es halt nochmal, ich aber für mich ist das jetzt / und vor allem, was ist / also ich kann mich wirklich / ich kann mich im Moment überhaupt nicht beherrschen.“ (Klientinnenaussage, Coachingsitzung 2016_1119a_01\#8, Zeitstempel \#00:06:57-8\#)

Dass der Umgang mit Unklarheit eines der zentralen Themen arbeitsweltlicher Beratung ist und Coaching als Klärungshilfe bei Problemen der Kommunikation und zwischenmenschlichen Beziehungsgestaltung, der Arbeitsbelastung und in Veränderungs- und Entwicklungsprozessen dienen kann, scheint unstrittig (vgl. Kapitel 2.1).

Unklarheit wurde im Rahmen der hier durchgeführten Task Analyse und der Festlegung des Task Markers (vgl. Kapitel 6.4) phänomenologisch anhand der im Coaching beobachtbaren Verhaltensweisen beschrieben:

- Unklarheit kann sich zeigen als Unsicherheit oder Irritation, die auch Gefühle oder Fragen der Haltung oder Motivation tangiert

- Es wird, mehr oder weniger explizit, ein Klärungsanliegen formuliert, verbunden mit dem Wunsch nach einer Veränderung

- Hinweise auf Unklarheit ergeben sich nicht nur aus den unmittelbaren verbalen Äußerungen, sondern oft auch aus Verhaltensweisen wie Innehalten, zögerlichem Formulieren oder Gesprächsverlagerungen.

Dass Unklarheit ein häufig auftretendes Phänomen im Coaching ist, lässt sich auch anhand der Komplexität der Probleme erklären, die in der Arbeitswelt auftauchen. Komplexität zeichnet sich aus durch 
„einen großen Umfang an beteiligten Bestimmungsstücken, deren Vernetztheit, einem eigendynamischen Geschehen, Intransparenz und Polytelie.“ (Starker \& Müller, 2018, S. 309)

Komplexität hat regelmäßig zu tun mit Multikausalitäten, Vernetzung vieler Zusammenhänge und Nebenwirkungen von Handlungen. Dabei lassen sich drei Bezugsebenen unterscheiden:

1. Sachbezug:

Im Kontext der oben zitierten Klientinnenaussage zeigt sich der Sachbezug darin, dass ihr Vorgesetzter, den die Klientin hier als „er“ bezeichnet, etwas von ihr forderte, was ihr Mühe bereitete. Komplex erscheint die Situation insofern, als dass sich ein Interessens- oder Strategiekonflikt zwischen dem Vorgesetzten und der Klientin vermuten lässt, und dass unterschiedliche Kollegen offensichtlich unterschiedlich mit den Anforderungen des Vorgesetzten umgehen. Es scheint also unterschiedliche Einschätzungen zu geben, wie die Sache zu handhaben ist. Starker \& Müller (ebd.) weisen darauf hin, dass hinsichtlich des Sachbezugs oft die Meinung vertreten wird, man müsse nur alle notwendigen Informationen besitzen, dann könne man Klarheit erlangen und sichere Entscheidungen treffen. Nach ihrer Einschätzung ist dies aber eine Illusion, denn Komplexität geht regelmäßig einher mit Uneindeutigkeit und einer unvollständigen Informationslage, die beim Menschen auf eine begrenzte Kapazität kognitiver Informationsverarbeitung und auf das Bedürfnis nach Konsistenz trifft, so dass die kognitiven Ressourcen des Individuums schnell überfordert sind.

2. Beziehungsebene:

Alle Coachinggespräche, die dieser Untersuchung zugrunde liegen, beinhalten auch Aspekte der Beziehungsgestaltung im Arbeitskontext. Im oben zitierten Beispiel geht es um die Arbeitsbeziehung zwischen der Klientin und ihrem Vorgesetzten, und am Rande auch um die Arbeitsbeziehung zwischen der Klientin und ihren Kollegen. Unklar ist dabei für die Klientin, wie sie die Interaktion ihres Vorgesetzten einschätzen soll, und demzufolge auch, wie sie die Beziehung zum Vorgesetzten gestalten soll. Wenn Scholl \& Kunert (2018, S. 265) die Interaktion als Grundlagenthema im Coaching bezeichnen, weisen sie damit genau auf diese Beziehungsebene hin, die oft ein eigendynamisches Geschehen entwickelt, das geprägt ist von Fragen des Vertrauens, der Kooperation, der Macht und Ohnmacht, der Nähe und Distanz, und auch der Sympathie und Antipathie. So entsteht aufgrund der Vielschichtigkeit der Interaktion bzw. Beziehungsgestaltung immer wieder ein gewisser Klärungsbedarf.

3. Emotionale Verarbeitung:

In der oben zitierten Klientinnenaussage wird bei dem Hinweis darauf, dass sie sich nicht mehr beherrschen könne, auch eine emotionale Ebene deutlich. Die Klientin sieht sich mit eigenen Emotionen konfrontiert, die ihr auf eine gewisse Art 
und Weise unangenehm sind. Starker \& Müller (2018, S. 310) weisen darauf hin, dass emotionale Aspekte wie Ärger, Angst, Freude oder Zufriedenheit mit jeweils typischen Denk- und Handlungscharakteristika einhergehen und daher besonders in komplexen oder unklaren Situationen Einfluss auf menschliches Entscheiden und Handeln nehmen. Betrachtet man die in Kapitel 3 und Kapitel 4 dargestellten psychologischen und neurowissenschaftlichen Grundlagen emotionaler Verarbeitung, lässt sich die Verarbeitung selbst ebenfalls als komplex bezeichnen, da sie unterschiedliche limbische Ebenen und sowohl vergangene als auch gegenwärtige Erfahrungen einbezieht. Daraus können Inkongruenzen zwischen den unterschiedlichen Verarbeitungsebenen entstehen, die als Unklarheit empfunden werden.

Vor dem Hintergrund der Komplexität wird deutlich, dass Coachinganliegen, wenn sie mit Unklarheit verbunden sind, nicht allein durch kognitive Anstrengungen im Sinn sachorientierter Überlegungen und auch nicht allein durch das Ausagieren affektiv-emotional gesteuerter Impulse bearbeitet werden können. Vielmehr bedarf es der Einbeziehung aller menschlichen Verarbeitungsebenen, aus neurowissenschaftlicher Sicht also der limbischen Ebenen ebenso wie der kognitiv-sprachlichen Ebenen, bzw. aus Sicht der humanistischen Psychologie der Einbeziehung sowohl aktueller affektiver und emotionaler als auch kognitiver Prozesse. Das Konzept des Experiencing und das im Rahmen der Task Analyse erarbeitete empirischtheoretische Modell beschreiben einen Prozess der Klärung, bei dem das Selbsterleben eine zentrale Ressource darstellt. Der Vorteil dieser Ressource kann darin gesehen werden, dass sie die unterschiedlichen Ebenen der Verarbeitung miteinander verbindet und eine Kongruenz zwischen kognitiven und affektiv-emotionalen Aspekten ermöglicht. Durch die Fokussierung auf das Selbsterleben entsteht bei einem gelingenden Prozess eine gewisse Distanzierung vom Umfeld, so dass auch die Beziehungsebene differenziert bearbeitet werden kann. Ergänzend kann die Arbeit mit dem Selbsterleben dem Klienten ermöglichen, gerade in komplexen Sachbezügen Aspekte der affektiv-emotionalen Informationsverarbeitung zu nutzen, um mehr Klarheit zu erhalten.

In Kapitel 4.2 wurde im Kontext der Neurowissenschaften bereits darauf hingewiesen, dass Selbsterleben nicht gleichzusetzen ist mit Selbstreflexion. Selbstreflexion spielt zwar im Coaching eine wichtige Rolle, indem sie den Klienten bei der Entwicklung und Veränderung seines Selbstkonzepts unterstützt (Greif \& Rauen, 2018, S. 523). Sie beruht ebenso wie das Selbsterleben auf der Selbstbeobachtung des Klienten, allerdings beobachtet der Klient sich im Rahmen der Selbstreflexion eher von einem äußeren Bezugspunkt aus. Wenn es dem Klienten dann gelingt, seine eigene Beobachterposition im Sinn einer Selbstexploration nach innen zu verlagern, kommt es zu Selbsterleben: Hier liegt der Bezugspunkt nicht außerhalb der eigenen Person, sondern quasi in der eigenen Person, es geht mehr um das (innere) Spüren als um das (äußere) Beschreiben, mehr um das aktuelle Erleben als um die Reflexion zurückliegender oder zukünftiger Gegebenheiten, mehr um einen sich selbst verändernden Prozess 
als um eine Zustandsbeschreibung. Selbstexploration und Selbsterleben stehen damit in einem engen Zusammenhang.

\subsection{Der Felt Sense als gefühlte Bedeutung}

„Aber ich merke gerade, wie das in mir pocht, ja? (Coach: Ja. Ja dann sag noch so zwei, drei Sätze dazu, was heißt das, das Pochen?) Dass ich immer noch unglaublich wütend bin. Und aber auch, auch eigentlich fassungslos. Also ja, dass ich merk "Boah, ich habe mir das alles bieten lassen. Hallo! Geht's noch?". Und mir ist das überhaupt nicht aufgefallen bis eben, dass die genau wieder dieses Schema bedient haben, auf dem ich so ansprechbar bin. Auf dem man mich so gut treffen kann.“ (Klientinnenaussage, Coachinggespräch 2017_1805_01\#1, Zeitstempel \#00:56:414\#)

Auf die Problematik der Konzeption Gendlins zum Felt Sense wurde bereits hingewiesen (vgl. Kapitel 3.5), und im Zusammenhang mit der theoretischen Rückbindung des hier erarbeiteten empirisch-theoretischen Veränderungsmodells wurde die Problematik der Symbolisierung, der im Zusammenhang mit dem Felt Sense eine wichtige Rolle zukommt, deutlich (vgl. Kapitel 6.8.5). Insofern kann der Felt Sense, wie er im Focusing-Konzept dargestellt ist, nicht als psychologisches Konstrukt bestätigt werden. Gleichzeitig lässt sich der im empirisch-theoretischen Modell abgebildete Experiencingprozess anhand der experienziellen Theorie (Sachse, 2011; vgl. Kapitel 3.5) im Sinn eines Übersetzungsprozesses affektiver Schemata nachvollziehen. Wenn es gelingt, die affektiven Schemata der Verarbeitung zu aktivieren und ihre Bedeutung auch kognitiv abzubilden, können sich daraus neue Erkenntnisse und aktivierende Momente für den Klienten ergeben. Dies zeigt auch beispielhaft die oben zitierte Klientinnenaussage und der sich daraus ergebende Veränderungs- und Lösungsprozess: Aus dem aktuellen Körperspüren heraus erhält die Klientin Zugang zu einer starken Emotion und deren Bedeutung, so dass sie daraus einen Lösungsweg erarbeiten kann. Insofern wäre es ein Verlust für die Praxis des Coaching, das Körperspüren, das aktuelle Experiencing und das Herausarbeiten der Bedeutung desselben nicht zu berücksichtigen.

Insgesamt lässt sich bezüglich des Felt Sense folgende Conclusio ableiten: Der Begriff Felt Sense kann als Umschreibung für die Aktivierung affektiver Schemata, das Explizieren dieser Schemata und das Herausarbeiten eines kognitiven Äquivalents zum Schema verstanden werden (vgl. Sachse et al, 1992, S. 49). Affektive Schemata können auch körperlich spürbar werden, so dass das Körperspüren und Körpererleben als Zugang zum Felt Sense genutzt werden kann. Von zentraler Bedeutung ist dann, nicht beim reinen Körperspüren zu verweilen, sondern dieses aus Ausgangpunkt für das Erarbeiten einer kognitiven Bedeutung zu nutzen. Geschieht dies nicht in ausreichendem Maße, besteht, wie in Kapitel 4.2 ausgeführt und wie auch im ersten kontrastierenden Beispiel (Episode 2016_1119a_01\#1) erkennbar, die Gefahr einer Verfestigung belastender Empfindungen. Dies korrespondiert auch mit einem Eintrag, den 
der Autor im Rahmen der Durchsicht der Transkripte und des Sampling in seinem Forschungstagebuch machte:

„Zu den nicht weiter berücksichtigten Prozessen: es gibt da einige mit EXP Rating 4: Langes Verweilen bei Stufe 4 scheint eher kontraproduktiv zu sein und zu einer Stagnation zu führen. Körperbezug und Gefühlsebene ohne das weitere Erarbeiten der Bedeutung bringt nicht voran.“

Wird der Begriff Felt Sense nun nicht im Sinn eines psychologischen Konstrukts genutzt, sondern mehr im Sinn einer illustrativen Umschreibung, weist die deutsche Übersetzung ,gefühlte Bedeutung“" auf zwei Aspekte hin, die Voraussetzung für einen gelingenden Experiencing-Prozess sind:

- Gefühle, vor allem die körperlich gespürten Gefühle, sind als Informationsträger nutzbar, als hochinferente, aber implizite, nicht vollständig explizierbare Wissensform (Sachse \& Langens, 2014, sh. Kapitel 3.1)

- Um zu einem vorantragenden Prozess beizutragen, müssen Gefühle mit einer Bedeutung im Sinn einer kognitiven Übersetzung des affektiven Schemas versehen werden (sh. Kapitel 3.5). Ein reines Erarbeiten des Gefühls würde noch keinen Prozess initiieren. Die Übersetzung des affektiven Schemas kann auf verschiedene Art und Weise geschehen: durch ein Symbol, eine Metapher oder eine sonstige Abbildung des gefühlten „Etwas“.

\subsection{Vom Felt Sense zum Felt Shift}

„Das müsste eigentlich relativ...leicht möglich sein. ......Es ist schon einmal erleichternder, der Gedanke daran..." (Klientinnenaussage, Coachingprozess 2016_1119a_01\#8, Zeitstempel \#00:54:03-2\#)

Die Bedeutung des Felt Shift als Ergebnis eines Veränderungsprozesses wird in der Coachingforschung von anderen Autoren bestätigt, wenn sie im Kontext intraindividueller Veränderung von Critical Moments (De Haan \& Nieß, 2012, 2018), Bedeutsamen Momenten (Wegener, 2019) oder Aha-Erlebnissen (Greif \& Riemenschneider-Greif, 2018) sprechen (sh. Kapitel 2.3). Im Experiencing-Konzept wird ein Felt Shift definiert als eine gefühlte Erleichterung bzw. als ein Moment des Verstehens oder der Klärung, in dessen Kontext eine körperlich fühlbare Empfindungsänderung, eintritt. Die idealtypischen Episoden 2017_2119_03\#3 und 2016_1119a_01\#8 geben anschauliche Beispiele, wie diese Art der Klärung auch eine direkte Replik auf zuvor formulierte Problem-Aspekte sein kann: während die Klientin zu Beginn der dritten idealtypischen Episode formuliert „,es macht mir Angst“, sagt sie im Rahmen der Task Finish „es macht mir keine Angst“. Ein ähnliches Beispiel findet sich in der zweiten idealtypischen Episode: hier ist anfangs vom Gefangensein (Zustand ohne Wahlfreiheit) die Rede, und die Replik im Rahmen der Task Finish ist: „ich kann es mir aussuchen“ (Zustand mit Wahlfreiheit). 
Aus dem empirisch-theoretischen Veränderungsmodell wird deutlich, dass der Felt Shift zum einen am Ende des intrapersonalen Veränderungsprozesses steht, und dass zum anderen sein Nutzen darin besteht, dass er zu einem weiteren Schritt überleitet, in dem eine Handlungsoption erarbeitet wird bzw. eine Bezugnahme auf das Umfeld stattfindet. Damit kann davon ausgegangen werden, dass die intrapersonale Veränderung nicht nur eine gewisse Erleichterung bewirkt, sondern vor allem auch eine für den Klienten stimmige Veränderung seines thematisierten Problems initiiert.

Aus den Modellen der kontrastierenden Prozesse wird erkennbar, dass der Felt Sense nicht automatisch einen Felt Shift nach sich zieht, sondern dass dazu ein entsprechender internaler Prozess beim Klienten notwendig ist. Kritisch für diesen internalen Prozess scheinen vor allem zwei Faktoren zu sein:

1. Distanzierung zum Umfeld:

Besonders das empirische Modell zur ersten kontrastierenden Episode, aber auch das Modell zur dritten kontrastierenden Episode zeigen einen deutlichen Unterschied zu den Modellen der idealtypischen Prozesse auf. Im Rahmen der idealtypischen Prozesse nimmt der Klient eine Positionierung zum Umfeld vor, die mit einer Distanzierung gegenüber den problematischen Verhaltensweisen des Umfelds einher geht. In den kontrastierenden Episoden ist eher eine intensive Beschäftigung mit dem Verhalten und dem Erleben des Umfelds zu beobachten, und damit zusammenhängend auch eine gewisse Identifizierung mit dem Umfeld. Aus dieser Beobachtung wird abgeleitet, dass die Distanzierung zum Umfeld eine wichtige Bedingung für einen gelingenden Experiencing-Prozess ist. Mit Distanzierung ist nicht gemeint, dass der Klient einen aktiven Konflikt mit dem Umfeld beschreiben muss oder eine allgemeine Distanz zum Umfeld einhalten sollte. Vielmehr geht es darum, dass er in der Lage ist, problematische Verhaltensweisen des Umfelds, die sich auf sein Thema oder sein Problem auswirken, auch als solche zu erkennen, diese dann aber nicht weiter intensiv zu explorieren, sondern zunächst als Problembeschreibung stehen zu lassen.

2. Verweilen beim Selbsterleben:

Während es bei der Distanzierung vom Umfeld eher um eine inhaltliche Positionierung geht, kennzeichnet das Verweilen beim Selbsterleben die Aufmerksamkeitslenkung im Coachingprozess selbst. Der Vergleich der idealtypischen und der kontrastierenden Prozesse legt nahe, dass das Verweilen beim Selbsterleben ein wesentliches Merkmal für gelingende Prozesse ist. Dort, wo Klienten ihre Aufmerksamkeit entweder nur kurz dem Selbsterleben zuwenden oder wo das Umfeld durchgehend eine zentrale Rolle einnimmt, kann das Selbsterleben weniger für eine Problemlösung genutzt werden.

Aus personzentrierter und experienzieller Sicht bestätigen diese Aspekte die Bedeutsamkeit 
der Selbstexploration. Diese ist entscheidend für das Selbsterleben und das Explizieren gefühlter Bedeutung. Selbstexploration wird, wie oben (Kapitel 7.1) beschrieben, verstanden als aktive Auseinandersetzung mit dem inneren Erleben und dessen Bedeutung und geht nach dem personzentrierten bzw. experienziellen Konzept regelmäßig einher mit einem Zugewinn emotionaler Selbstregulationsfähigkeit (vgl. Kapitel 3.1).

Wie sich ein hohes Maß an Selbstexploration im Vergleich zu einem niedrigen Maß an Selbstexploration auswirkt, lässt sich am Vergleich der ersten idealtypischen Episode mit der ersten kontrastierenden Episode nachvollziehen: Die Unterscheidung besteht nicht nur darin, dass die Klientin im idealtypischen Beispiel ein Experiencing der Stufe 6 (EXP 6) erreicht, während die Klientin im kontrastierenden Beispiel nur und nur für kurze Zeit die Stufe 5 (EXP 5) erreicht, sich aber ansonsten zwischen Stufe 2 und Stufe 4 (EXP 2 und EXP 4) bewegt. Kennzeichnend für den idealtypischen Prozess ist vor allem, dass die Klientin über längere Zeit ausschließlich über ihr inneres Erleben und nicht über ihr Umfeld spricht, und dass sie das innere Erleben differenziert beschreibt. Dies geht so weit, dass die Klientin nicht der Anregung des Coachs folgt, als dieser sie von der Selbstexploration wegführen möchte (Gespräch 2017_1805_01\#1, Zeitstempel \#00:57:54-2\#), sondern weiter beim Selbsterleben verweilt. Offensichtlich haben gerade das körperliche Spüren und die gefühlte Bedeutung ihr Interesse und ihre Energie aktiviert, so dass sie ihre ganze Aufmerksamkeit dem Explorieren des Erlebens widmet und infolgedessen dann auch zu einer Lösung kommt.

Im kontrastierenden Beispiel ist eine solche Phase nicht erkennbar, vielmehr nimmt die Klientin immer wieder Bezug zum Umfeld, und zwar nicht in Form einer Distanzierung, sondern in Form einer Auseinandersetzung mit der Befindlichkeit des Umfelds. Das führt zu einer Unterbrechung im Experiencingprozess, die sich darin zeigt, dass das Experiencing der Stufe 4 (EXP 4) zwischenzeitlich von der niedrigen Experiencing-Stufe 2 (EXP 2) abgelöst wird, die zwar durch ein sachliches Interesse, nicht aber durch eine Bezugnahme auf das eigene Fühlen gekennzeichnet ist. Als die Klientin dann wieder zu ihrem eigenen Erleben zurückkehrt (EXP 4 und EXP 5), gelingt es ihr kurzzeitig, eine Metapher für ihr Erleben zu erarbeiten (,gefangen“) und in diesem Zusammenhang auch eigene Ressourcen („Ausweg“) und neue Energien (,dann kann ich auch was machen“) zu aktivieren (Gespräch 2016_1119a_01\#1, Zeitstempel \#00:45:51-1\#). Sie kehrt nach einer kurzzeitigen Ressourcenaktivierung dann aber - auf Nachfrage der Coach - wieder zu einer noch differenzierteren Beschreibung ihres Belastungserlebens zurück, verliert dadurch die Ressourcen und die eigenen Handlungsoptionen aus dem Blick und kann diese später auch nicht mehr aktivieren. Selbstexploration findet hier nur partiell statt. Die Klientin beschäftigt sich zwar mit ihren Gefühlen bzw. ihrem Belastungserleben, es findet aber keine Differenzierung des Erlebens statt, und diese wäre notwendig, um über das Selbsterleben zum Felt Shift zu gelangen. 


\subsection{Trilemma-Situation als Ausgangspunkt}

Aus den bisherigen Überlegungen lassen sich Indikationen ableiten, in welchen Fällen der Unklarheit ein experiencingorientierter Prozess besonders hilfreich sein kann. Dazu soll die Anfangsphase der Modelle nochmals genauer betrachtet werden: Im Gegensatz zu den kontrastierenden Modellen weisen die idealtypischen Beispiele eine Gemeinsamkeit auf, die im gemeinsamen Modell der idealtypischen Beispiele dargestellt ist als eine Situationsbeschreibung, die einerseits ein problematisches Verhalten des Umfelds mit Bezug zur eigenen Person beinhaltet und andererseits ein persönliches Spannungserleben, das auf irgendeine Art kognitiv und affektiv wahrgenommen wird. Dieses Spannungserleben als Missverhältnis zwischen drei Komponenten lässt sich auch als Trilemma darstellen (Abbildung 11):

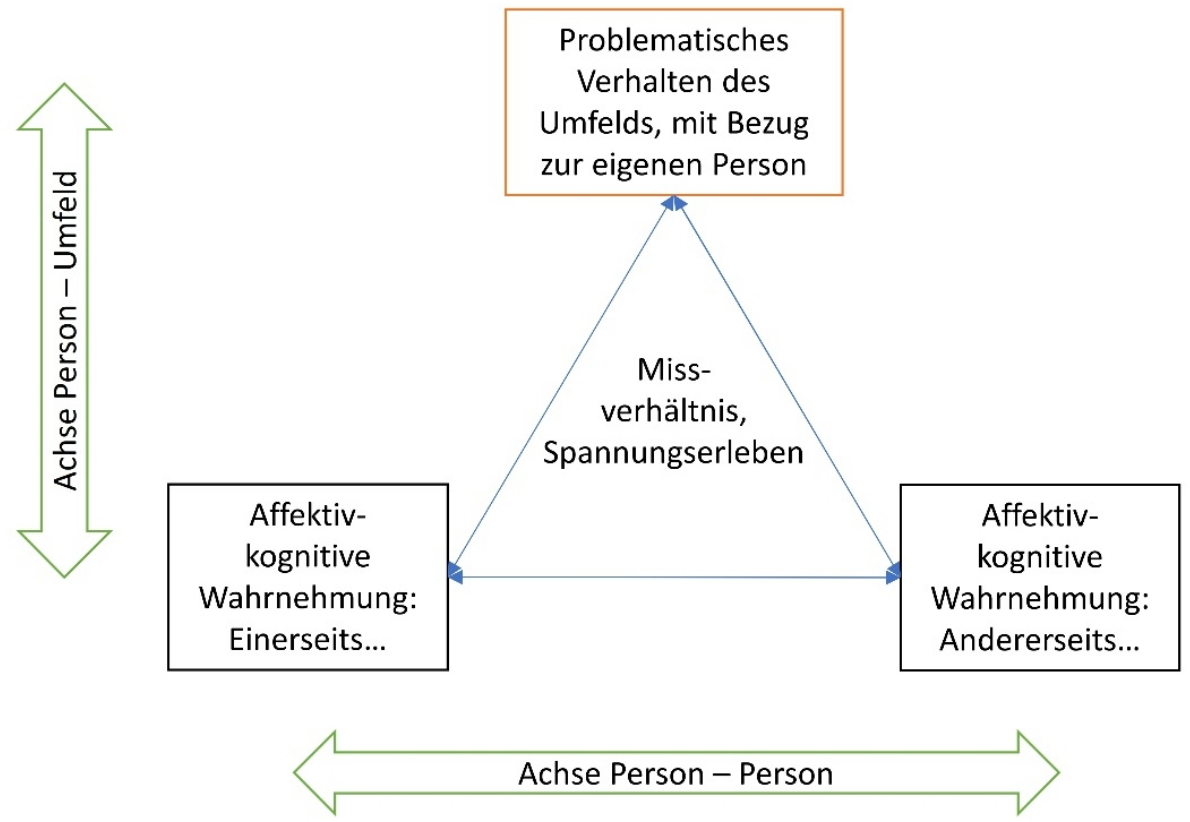

Abbildung 11: Trilemma

Wie sich dieses Missverhältnis konkret zeigen kann, verdeutlichen die Beispiele der drei idealtypischen Episoden. Es lassen sich folgende Trilemma-Situationen abbilden (Abbildungen 12-14): 


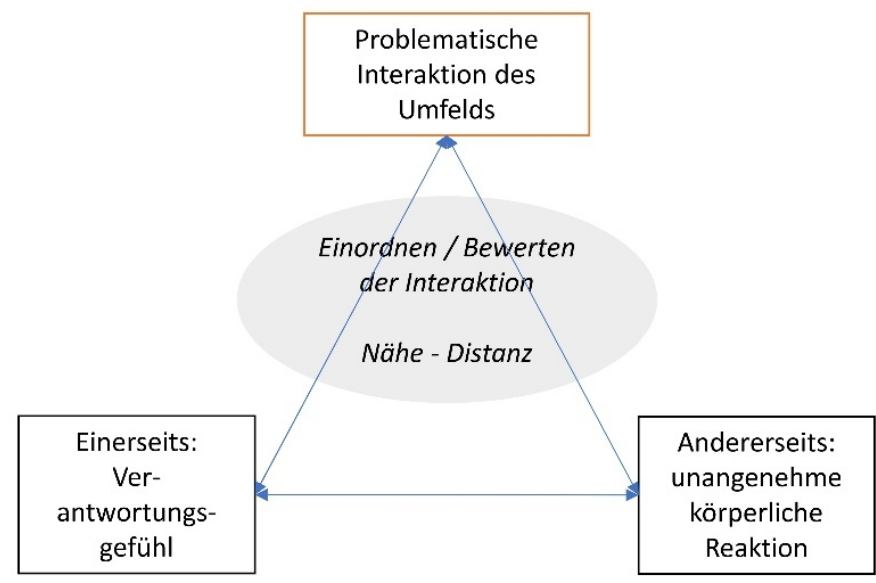

Abbildung 12: Trilemma-Beispiel aus Episode 2017_1805_01\#1

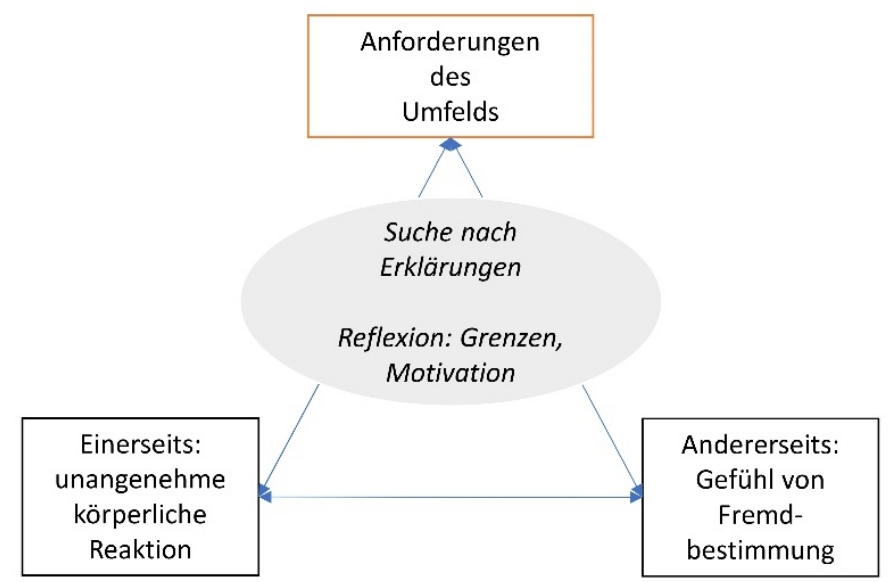

Abbildung 13: Trilemma-Beispiel aus Episode 2016_1119a_01\#8

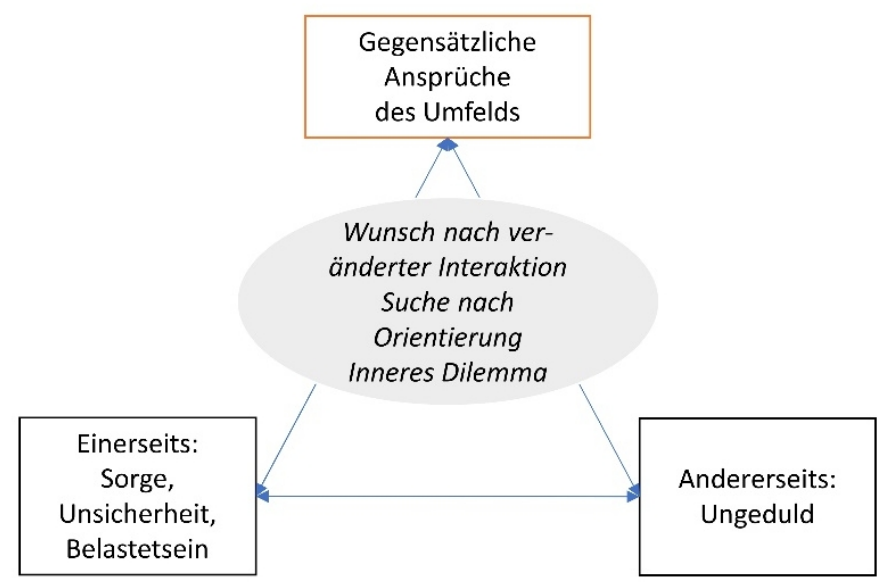

Abbildung 14: Trilemma-Beispiel aus Episode 2017_2119_03\#3 
Typisch für das Trilemma scheint, dass das Spannungserleben oder auch die Unklarheit zunächst alle drei Seiten des dargestellten Dreiecks betrifft und eine einfache Lösung im Sinn einer Kompromissfindung oder einer eindeutigen Positionierung nicht zu finden ist. Anhand der Trilemma-Beispiele ist erkennbar, dass unterschiedliche affektiv-kognitive Wahrnehmungen möglich sind, die sich nicht zwingend als Gegensätze gegenüberstehen müssen, sich aber hinsichtlich ihrer Beziehung zum Umfeld unterscheiden.

Wenn im Coaching solche Situationsbeschreibungen auftauchen, die sowohl eine Spannung zwischen der eigenen Person des Klienten und dem Umfeld (Achse Person-Umfeld) als auch eine Spannung im Erleben der Person selbst (Achse Person-Person) andeuten, kann das als Indikation für experiencingorientiertes Vorgehen genutzt werden.

Bei den kontrastierenden Modellen fällt auf, dass die beiden Spannungsachsen Person-Umfeld und Person-Person in der Situationsbeschreibung nicht deutlich zu beschreiben sind. Dies wird als Hinweis darauf verstanden, dass im experiencingorientierten Coaching bereits bei der Situationsbeschreibung darauf zu achten ist, inwieweit eine eventuelle Trilemma-Problematik auch dezidiert und differenziert herausgearbeitet werden kann.

\subsection{Indikationen und Kontraindikationen}

Es wurde bereits dargestellt, dass Trilemma-Situationen als Ausgangspunkt für experiencingorientiertes Coaching genutzt werden können. Aus den bisherigen Überlegungen ergeben sich weitere Hinweise, wann Experiencing im Coaching besonders hilfreich sein kann. So kann auf folgende Indikationen geachtet werden:

- Der Coachee beschreibt eine Unklarheit, die mit intensiven Gefühlsregungen einhergeht und / oder für die er bisher noch kein tieferes Verständnis entwickeln konnte, für die er sich aber eine Veränderung wünscht

- Die Unklarheit lässt sich als Trilemma-Problematik beschreiben anhand der Spannungsachsen Person-Umfeld und Person-Person, und die Beschreibung der Trilemma-Problematik bezieht sich sowohl auf kognitive als auch auf affektive Aspekte

- Zusätzlich zu den verbalen Inhalten kann auch das allgemeine Verhalten des Coachee Hinweisreize zum experiencingorientierten Vorgehen geben: Wenn der Klient sein Verhalten im Coaching ändert, häufiger schweigt, nach Worten sucht, um ein Gefühl zu beschreiben, um Metaphern ringt oder körperliche Veränderungen zeigt, sollte dies die Aufmerksamkeit des Coachs wecken, bei den verbalen Inhalten besonders auf Unklarheiten und Trilemma-Problematiken zu achten (vgl. Kapitel 3).

Ergänzend sollte der Coachee über eine gewisse Bereitschaft und eine gewisse Grundkompetenz zur Selbstexploration verfügen. Das schließt die Möglichkeit ein, sich vom Umfeld zu 
distanzieren und beim Selbsterleben zu verweilen. Besonders Sachse (1992, S. 22, vgl. Kapitel 3.4) weist darauf hin, dass die Fähigkeit zur Selbstexploration nicht bei allen Menschen gleich ausgeprägt ist, und dass ein niedriges Maß an Selbstexplorationsfähigkeit das Explizieren eines Felt Sense erschwert. Auch Abwehr, Widerstand oder die Angst gegenüber der Beschäftigung mit bestimmten intrapersonalen Aspekten kann die Bereitschaft zur Selbstexploration einschränken. Wie stark die Bereitschaft zur Selbstexploration ausgeprägt ist oder inwiefern sie beeinträchtigt ist, lässt sich allerdings nicht vorab diagnostizieren, vielmehr wird sich das erst im Lauf des Prozesses zeigen.

Experiencing kann nicht in allen Situationen als Lösungsweg genutzt werden. So können sich im Coaching Aspekte ergeben, die als Kontraindikationen für experiencingorientiertes Vorgehen zu werten sind:

- Bei solchen Situationsbeschreibungen, die keine Unklarheit im Sinn einer Trilemma-Problematik beinhalten, scheint experiencingorientiertes Vorgehen nicht besonders hilfreich im Sinn einer Problemlösung oder Zielerreichung zu sein

- Reine Beschreibungen von Körpergefühlen (vgl. Non-Task-Marker Beispiel 1, Kapitel 6.4) ergeben noch keine hinreichende Indikation für einen ExperiencingProzess, solange sie nicht in Verbindung mit einem persönlichen Involviertsein und einer Unklarheit stehen

- In Kapitel 3.4 wurde darauf hingewiesen, dass Menschen mit psychosomatischen Beschwerden in der Regel weniger von einem Focusingprozess profitieren können als andere. Auch dieser Aspekt sollte im Kontext des Körperspürens berücksichtigt werden: Beschreibungen von Körpererleben, die eher auf psychosomatische Beschwerden hinweisen, sollten nicht als Körpererleben im Sinn des Experiencing missverstanden werden

- Situationsbeschreibungen, die nicht mit einem auf die eigene Person bezogenen Veränderungswunsch einhergehen, lassen vermuten, dass kein Anreiz vorliegt, der den Klienten zu einer vertieften Selbstexploration motiviert

- Auch wenn Experiencing und speziell Focusing augenscheinlich eine gewisse Nähe zu therapeutischen Prozessen hat, sollte klar sein, dass ein therapeutischer Bedarf auch im experienziellen Coaching nicht abgedeckt werden kann. Deutlich wird das am Beispiel der zweiten kontrastierenden Episode (Coachinggespräch 2015_2007_02\#1). Die Klientin beschreibt dort im Rahmen ihrer Selbstexploration ein Defiziterleben, eine Kraftlosigkeit und Hilfsbedürftigkeit, die darauf schließen lassen, dass die Belastung im Selbsterleben aktuell so stark dominiert, dass ihre üblichen Möglichkeiten zur Selbstaktualisierung nicht greifen. Im Beispiel nimmt der Coach sowohl die Problemstellung als auch die Kontraindikation ernst, indem er der Klientin empfiehlt, sich in psychotherapeutische Behandlung zu begeben.

$\mathrm{Zu}$ beachten ist, dass die Indikationen und Kontraindikationen für experiencingorientiertes 
Coaching überwiegend nicht digital im Sinn von entweder/oder betrachtet werden können, sondern eher als Kontinuum von Faktoren zu sehen sind, die mehr oder weniger deutlich ausgeprägt sind. Dies gilt insbesondere für die Trilemma-Problematik, die Selbstexploration und die Distanzierung vom Umfeld. Hier wird auch maßgeblich sein, inwieweit und wie der Coach den Coachee in seinem Coachingprozess unterstützen kann.

\subsection{Vom Trilemma zur Dialektik innerer Erlebensanteile}

Der Prozess vom Felt Sense zum Felt Shift kann in Anlehnung an Greenbergs prozess-experienzielles emotionsfokussiertes Konzept auch als dialektischer Prozess innerer Erlebensanteile veranschaulicht werden. Ausgehend von der Trilemma-Situation, besteht die Besonderheit der Verarbeitung darin, dass es dem Klienten idealerweise gelingt, sich im Wesentlichen auf die Achse Person-Person (sh. Abbildung 11) zu konzentrieren und ein Experiencing-Niveau der Stufe 4 oder höher zu erreichen (EXP $\geq 4)$. Dabei bringt er seine inneren Erlebensanteile, seine unterschiedlichen affektiv-kognitiven Wahrnehmungen, sein Körpererleben, seine Gefühle, seine Bedürfnisse und seine Ressourcen in einen inneren Dialog miteinander. Dieser Dialog findet in einer Art dialektischer Auseinandersetzung statt, es kommen also gegebenenfalls durchaus gegensätzliche Elemente zur Sprache, die aber nicht polarisierend im Sinn einer Entweder-Oder-Entscheidung wirken, sondern sich eher gegenseitig ergänzen und im Idealfall zu einem neuen inneren Erleben oder zu einem besseren Verständnis führen. In Gendlins Terminologie könnte an dieser Stelle auch von „kreuzen“ gesprochen werden, im Sinn eines Dialogs unterschiedlicher Einsichten oder Empfindungen, aus dem Neues erwachsen kann.

Beispiele, worum es bei dieser Dialektik innerer Erlebensanteile gehen kann, ergeben sich aus den empirischen Modellen der idealtypischen Prozesse. Sie verdeutlichen, dass es dabei nicht in erster Linie um sich diametral gegenüber stehende Gegensätze geht, sondern um unterschiedliche Facetten inneren Erlebens, um unterschiedliche Motivationsstränge und Bedürfnisse, und im weiteren Verlauf der Verarbeitung auch um eine gewisse Pendelbewegung zwischen dem, was als schwierig und belastend erlebt wird und dem, was Ressourcen und Energie freisetzt:

\begin{tabular}{|l|l|}
\hline Einerseits... & Andererseits... \\
\hline Bedürfnis nach Nähe & Bedürfnis nach Distanz \\
\hline Verantwortungsübernahme für das Umfeld & Distanzierung gegenüber dem Umfeld \\
\hline affektiv gesteuerte Handlungsimpulse & $\begin{array}{l}\text { kognitiv gesteuerte, rationale Handlungsim- } \\
\text { pulse }\end{array}$ \\
\hline Fremdbestimmung & Orientierungslosigkeit \\
\hline Grenzen & Motivation \\
\hline
\end{tabular}




\begin{tabular}{|l|l|}
\hline Ressourcen & Überfordertsein \\
\hline Selbstfürsorge & Wut \\
\hline Gute Erfahrungen & Ungeduld \\
\hline
\end{tabular}

Tabelle 14: Dialektik innerer Erlebensanteile (Beispiele)

Für den nicht direkt beteiligten Beobachter erschließt sich aus den Einerseits-AndererseitsFacetten nicht immer eine Logik oder Systematik. Das scheint auch für den Prozess nicht notwendig zu sein, denn nicht alle Erlebensanteile des Klienten lassen sich kognitiv-rational erschließen. Wichtig scheint daher, dass der Coach dem Klienten den entsprechenden Freiraum und die notwendige Zeit für den inneren dialektischen Prozess zugesteht und die Aufmerksamkeit des Klienten nicht vorschnell auf andere Aspekte lenkt. Als Ergebnis der Dialektik innerer Erlebensanteile kommt der Klient dann im Idealfall zu einer veränderten Deutung oder Bewertung und kann auch unter Bezugnahme auf das Umfeld einen Lösungsansatz erarbeiten.

Auch dieser Prozess ist nicht als linear zu sehen, sondern es können mehrere Rückkoppelungsschleifen erforderlich sein, um zu einer erlebten Stimmigkeit zu gelangen. Kennzeichnend ist dabei eine Zunahme des Experiencing, wie das für die Stufen 5 und 6 der Experiencing-Skala (sh. Kapitel 4.6) beschrieben ist: Zunächst konzentriert sich der Klient auf den inneren Bezugsrahmen, erforscht innere Erlebnisse und kann dann einzelne Aspekte differenzieren. Infolgedessen können einzelne Erlebnisinhalte deutlicher werden, sich verändern und zur Grundlage für weitere Überlegungen werden.

\subsection{Ausstiegsmomente aus dem Experiencing}

Die untersuchten Beispiele zeigen, dass nicht jeder Experiencingprozess auch zu einem Felt Shift oder zu einer Lösung des bearbeiteten Problems führt. Die kontrastierenden Modelle geben Hinweise darauf, an welchen Stellen oder unter welchen Bedingungen ein Ausstieg aus dem Prozess besonders wahrscheinlich ist:

1. Der Ausstieg beginnt bereits vor dem Einstieg: Das empirische Modell zur dritten kontrastierenden Episode (2017_0118_01\#2) zeigt anschaulich, wie dieser „Ausstieg vor dem Einstieg“" sich darstellt. Zwar beschreibt die Klientin ein Dilemma und auch eine Unklarheit bezüglich des Umfelds, die Situationsbeschreibung beinhaltet aber keine Trilemma-Problematik im engeren Sinn. Hinzu kommt, dass bei einer Vertiefung der Situationsbeschreibung zwar eine Veränderung des Experiencing von Stufe 2 (EXP 2) hin zu Stufe 3 (EXP 3) stattfindet, dann aber keine weitere Vertiefung des Selbsterlebens erfolgt. Im Gegensatz dazu beinhaltet das theoretisch-empirische Modell eine Anfangssituation im Sinn der Trilemma-Prob- 
lematik und direkt im Anschluss im Rahmen der Vertiefung der Situationsbeschreibung ein Experiencing der Stufe 4 oder höher (EXP $\geq 4$ ). Wenn diese beiden Faktoren nicht gegeben sind, muss von einem Ausstieg bereits vor dem Einstieg in die Aufgabenbearbeitung ausgegangen werden, auch wenn das zu bearbeitende Anliegen an sich im Rahmen von Experiencing bearbeitbar wäre.

2. Ein weiterer Aspekt, der einem gelingenden Prozess entgegen steht, ist die fehlende Distanzierung vom Umfeld. Dies ist am empirischen Modell zur ersten kontrastierenden Episode (2016_1119a_01\#1) gut erkennbar: Statt einer Distanzierung findet in der Phase der vertieften Situationsbeschreibung eine Identifikation mit dem Erleben des Umfelds statt, und in der Folge kommt es nicht zu einem Verweilen beim Selbsterleben. Auch im empirischen Modell zur dritten kontrastierenden Episode (2017_0118_01\#2) beschäftigt sich die Klientin durchgehend mit dem Verhalten des Umfelds und kommt nicht zu der Phase des Selbsterlebens. Solange ein Klient also wenig Distanz zum Umfeld einnehmen kann oder sich aus anderen Gründen intensiv mit dem Umfeld beschäftigt, muss dies als Hindernis für einen weiteren konstruktiven Prozess betrachtet werden.

3. Wenn emotionale Belastungen so schwer wiegen, dass sie trotz gewisser Bemühungen nicht im Sinn eines Felt Sense wahrgenommen und in ihrer Bedeutung expliziert werden können, ist der vorantragende Prozess des Experiencing gefährdet. Dies wird am empirischen Modell zur zweiten kontrastierenden Episode (2015_2007_02\#1) erkennbar. Verweilt der Coachee dann trotzdem beim inneren Erleben, besteht die Gefahr der Verfestigung der Belastung, weil ihr keine konstruktive Bedeutung beigemessen werden kann. In diesem Fall scheint es nicht angebracht, weiter beim Selbsterleben zu verweilen, vielmehr scheint ein Themenwechsel sinnvoll, und vor allem die Klärung, wie und in welchem Rahmen die belastende Problematik bearbeitet werden kann.

\subsection{Coachverhalten und Methodengebrauch}

Es war nicht Gegenstand dieser Arbeit, das Verhalten und die Kommunikation von Coachs in experienziellen Prozessen zu untersuchen. Trotzdem ergeben sich aus dem empirisch-theoretischen Modell zur Veränderung und aus den daraus abgeleiteten Implikationen Hinweise zum Coachverhalten und zum Methodengebrauch, die hier zur Diskussion gestellt werden sollen.

Grundlegend für die hilfreiche Begleitung von intrapersonaler Veränderung scheint weniger der Methodeneinsatz zu sein, sondern mehr das eigene mentale Modell bzw. die kognitive Karte des Coachs. Folgt diese dem bottom-up-Modell (vgl. Kapitel 6.5), wird er seine Aufmerksamkeit eher auf Selbsterlebensprozesse richten. Um diese Prozesse auch für sich selbst zu strukturieren, kann der Coach die Konstrukte Felt Sense und Felt Shift nutzen: es gilt, zunächst die gefühlte Bedeutung des Körpererlebens und der Gefühle zu erarbeiten und dann 
so lange beim Selbsterleben zu verweilen, bis sich ein Felt Shift einstellt. Ohne Felt Shift ist der Prozess unvollständig.

\subsubsection{Personzentrierte Beziehungsvariablen}

Über die personzentrierten Beziehungsvariablen Kongruenz, Wertschätzung und Empathie existieren zahlreiche Veröffentlichungen (vgl. Kapitel 3.1), und es erscheint fast müßig, sie hier nochmals zu erwähnen. Im Kontext intrapersonaler Veränderung kommt ihnen insofern eine wichtige Bedeutung $\mathrm{zu}$, als dass ein gelingender Experiencingprozess weniger vom jeweiligen Methodeneinsatz abhängig ist als mehr von einem wohlwollenden Rahmen, der dem Klienten ermöglicht, in sich selbst Zusammenhänge und Ressourcen zu entdecken, ohne dass ihm dazu von außen Vorgaben gemacht oder Interpretationen angeboten werden. Es geht bei diesen Beziehungsvariablen also in erster Linie um Freiraum zur Selbstentfaltung, um ein Beziehungsangebot, das eine schrittweise Selbstöffnung und eine selbstgesteuerte und klärende Auseinandersetzung mit dem inneren Erleben ermöglicht und dadurch das autonome Entwickeln von Handlungs- und Lösungsstrategien fördert.

\subsubsection{Trilemma als Ausgangspunkt}

Auch wenn im experienziellen Vorgehen großer Wert auf die Prozessorientierung gelegt wird und eine gewisse Zirkularität als selbstverständlich erachtet werden muss, sollte dies nicht mit Strukturlosigkeit gleichgesetzt werden, auch nicht bei der Bearbeitung von Unklarheit. Die Struktur wird hier aber weniger vom Zielpunkt vorgegeben, sondern vom Ausgangspunkt. Auf Basis der bisherigen Überlegungen lässt sich dieser Ausgangspunkt definieren als eine Situation, in der der Coachee eine Trilemma-Problematik beschreibt.

Aufgabe des Coachs ist dabei, den Klienten darin zu unterstützen, die Eckpunkte und Spannungsverhältnisse des Trilemmas möglichst deutlich herauszuarbeiten. Werden diese Eckpunkte vom Klienten nicht deutlich erkannt, könnte die weitere experiencing-orientierte Bearbeitung ins Stocken geraten. In diesem Fall könnte eine Rückkehr zum Ausgangspunkt indiziert sein. Beobachtet der Coach also, dass im Rahmen der Vertiefung der Situationsbeschreibung die Person-Umfeld-Achse nicht klar definiert ist, weil beispielsweise keine Positionierung zum Umfeld stattgefunden hat, wird es wichtig sein, dass der Coach den Klienten zur nochmaligen Reflexion dieser Beziehungsachse einlädt, bevor es zur Phase des Verweilens beim Selbsterleben kommt. Ähnliches gilt für die Person-Person-Achse: hier geht es zunächst darum, dass der Klient bei sich selbst wahrnehmen kann, dass „Etwas“ in seinem Inneren nicht stimmig ist, dass er also eine Art von Spannung oder Irritation erlebt, die auf unterschiedliche innere Anteile zurückzuführen ist. Im Vordergrund steht in dieser Phase allerdings nicht die Bearbeitung dieses „Etwas“, sondern lediglich dessen Wahrnehmung, so dass ein Bewusstsein für die Relevanz der Person-Person-Achse bzw. der inneren Erlebensanteile entsteht.

Bereits in dieser Phase des Prozesses gilt, was für alle folgenden Phasen ebenso von Bedeu- 
tung ist: der Fokus der Aufmerksamkeit liegt nicht auf Ereignissen oder Erlebnissen der Vergangenheit, sondern in der Gegenwart. Wichtig scheint daher, dass der Coach sich selbst immer wieder auf den aktuellen Moment konzentriert. Auch wenn in den Schilderungen des Klienten Vergangenheit und Gegenwart oft verschwimmen, weil der Klient die Vergangenheit in die Gegenwart hineinträgt (vgl. Gendlins Prozessmodell, Kapitel 3.5), richtet der Coach sein Augenmerk auf das gegenwärtige Erleben des Klienten.

\subsubsection{Raum zum Verweilen}

Ein zentraler Aspekt des Experiencing ist das Verweilen beim Selbsterleben. Es wurde bereits darauf hingewiesen, dass es auch möglich ist, dass Coachees trotz anderweitiger Interventionen der Coachs beim Selbsterleben verweilen. Zugespitzt formuliert, könnte für das Coaching abgeleitet werden, dass Coachs den Klienten zumindest nicht vom Verweilen beim Selbsterleben abhalten sollten, indem sie in dieser Prozessphase beispielsweise Fragen zum Umfeld stellen, vorschnell auf die Suche nach Ressourcen gehen oder einen Themenwechsel vorschlagen.

Auch wenn es nicht Gegenstand der Untersuchung war, fiel beim Hören der Audiographien der Gesamtstichprobe auf, dass Coachs nicht selten nach dem Spüren im Körper fragten, dass sich daraus aber kein vorwärts tragender Prozess ergab (sh. Anlage 2: Forschungstagebuch). Aus den untersuchten Prozessen wird deutlich, dass experiencingorientiertes Vorgehen nicht darauf zu verkürzen ist, dass das Körperspüren eben noch kurz gestreift wird, sondern dass es um eine vertiefte Auseinandersetzung mit dem aktuellen Selbsterleben geht. Gleichzeitig sollte sich der Coach bewusst sein, dass Selbsterleben neben dem für den Felt Sense zentralen Körperspüren noch über andere Kanäle exploriert werden kann. Beispiele hierfür finden sich in den empirischen Modellen, wo das Selbsterleben auch über das Beschreiben von Emotionen oder das Verwenden von Metaphern ausgedrückt wird. Folgt man der Systematik von Kern (2015, S. 137) zu den Modalitäten und Kanälen des Selbsterlebens, ergeben sich weitere Hinweise, welche Aspekte ergänzend zum Körperbezug eine Rolle beim Erkunden und Explizieren des eigenen Erlebens spielen können:

- $\quad$ kinestätischer Aspekt: Körperempfindungen und Handlungsimpulse

- $\quad$ visueller Aspekt: Bilder, Imaginationen

- $\quad$ akustischer Aspekt: innere Sätze, Töne, Melodien

- $\quad$ affektiver Aspekt: Emotionen

- $\quad$ Bedeutungsbildung: Gedanken.

Jeder dieser Aspekte kann einen Beitrag dazu leisten, das Selbsterleben zu fördern, solange er sich auf das gegenwärtige Geschehen bezieht und der Klient dabei einen inneren Bezugspunkt einnimmt. Die Rolle des Coachs besteht darin, den Klienten hierbei zu unterstützen. Dies geschieht, indem er den Raum und die Zeit dafür zur Verfügung stellt und sein eigenes Kommunikationsverhalten auf das Erleben des Klienten ausrichtet. Eine Orientierung hierzu geben die von Gendlin (1968) formulierten Regeln erlebensbezogenen Antwortens, die für 
Coachingprozesse folgendermaßen übertragen werden können:

- Wir achten auf Körpererleben und Gefühle und geben dem Klienten Raum, diese zu beschreiben

- Wir versuchen, die ,gefühlte Bedeutung“ dieses Erlebens zu explizieren, damit neue Aspekte sich entfalten können

- Wir schlagen verschiedene Richtungen der „gefühlten Bedeutung“ vor, um die Selbstexploration zu fördern und um einen erlebensmäßigen Fortschritt zu ermöglichen

- Wir folgen der Erlebensspur des Klienten. Wir gehen ihr nicht voraus, und wir bestimmen auch nicht, wie diese Erlebensspur auszusehen hat

- Wir versuchen dafür zu sorgen, dass das Erleben sich fortsetzt und vertieft

- Wir achten auf die Verlagerung des Bezugspunkts. Je mehr dieser sich nach innen verlagert, desto intensiver ist der experienzielle Prozess.

In der Begleitung von Focusingprozessen werden hierzu die Termini „Listening“ und „Guiding“ verwendet (Renn, 2016, S. 116). Listening kennzeichnet das Zuhören im Sinn dessen, dass der Coach möglichst nah am Erleben des Klienten ist und ihm quasi Schritt für Schritt folgt. Guiding im Sinn von Führen oder Lenken meint nicht, deutend oder interpretierend in die Prozessinhalte einzugreifen, sondern bezieht sich auf das Hinführen zum Impliziten, also zum Spüren und Fühlen, und darauf aufbauend ebenso auf das Hinführen zum Expliziten, also zum Herausarbeiten der Bedeutung des Erlebens. Sowohl für das Listening als auch für das Guiding wurden von verschiedenen Autoren Techniken beschrieben, die sich auch im Coaching anwenden lassen (z.B. Renn, 2016; Gendlin, 1996; Le Coutre, 2016).

Um Körpererleben angemessen adressieren zu können, kann es hilfreich für den Coach sein, sich der verschiedenen Zugangswege zum Körpererleben bewusst zu sein und gegebenenfalls entsprechende methodische Impulse zu setzen. Kern (2015, S. 137) nennt dazu unter anderem folgende Aspekte, die je nach Situation auch im Coaching umsetzbar sind:

- Körperwahrnehmung: Sie kann beispielsweise gefördert werden, indem der Klient seine Aufmerksamkeit auf Körperempfindungen richtet („Wie fühlt sich das an?“, „Wo im Körper ist etwas zu spüren?“), einen guten Ort im Körper sucht, gedanklich durch seinen Körper wandert und diesen von innen heraus beobachtet, sich den kleinen physiologischen Regungen (Kribbeln, Ziehen, Wärme, Kälte etc.) freundlich zuwendet oder eine Körperstelle in der Wahrnehmung möglichst genau beschreibt

- Atmung: Veränderungen in der Atmung können als Körpersignal genutzt werden, der Atem kann mit einem Thema verbunden werden, und es können Atemübungen angeboten werden

- Körperhaltung: der Klient kann eingeladen werden, die aktuelle Körperhaltung innerlich zu erspüren („Wie fühlt sich das an, so dazustehen?"), eine bestimmte Haltung zu verstärken bzw. zu übertreiben und dadurch zu verdeutlichen, oder 
eine gegenteilige Haltung einzunehmen und ihr nachzuspüren

- Bewegung: der Coach kann eine unwillkürliche Geste oder Bewegung ansprechen, nach einem Bewegungsimpuls fragen oder Ausdrucksbewegungen unterstützen.

\subsubsection{Prozessorientierung und Zielorientierung}

Im Kontext des Trilemmas wurde erkennbar, dass der experienzielle Prozess vom Ausgangspunkt bestimmt wird und nicht vom Zielpunkt. Dies scheint zunächst im Widerspruch zu stehen zu einem zielorientierten Vorgehen im Coaching, wie es beispielsweise in Greifs Strukturmodell (Greif, 2008, vgl. Kapitel 2.2.2) dargestellt und gefordert ist.

Hierzu ist anzumerken, dass ein experienzielles Vorgehen in einer bestimmten Episode den Coach nicht davon entbindet, immer wieder für eine Rückkoppelung des jeweils zur Bearbeitung stehenden Themas mit dem Gesamtanliegen und den Zielen des Coaching zu sorgen. In der Episode selbst wird der Arbeitsauftrag dann in der Tat zunächst vom Ausgangspunkt bestimmt, und der Zielpunkt ist insofern spezifiziert, als dass er an einer neu gewonnen Klarheit und damit einhergehenden Erleichterung (Felt Shift) erkennbar ist, die dann auch zu einer neuen Handlungsoption führt.

\subsubsection{Umgang mit Zweifeln und Unsicherheit}

Nicht immer führt die im Rahmen des Experiencing neu gewonnene Klarheit zu einer vollumfänglichen Lösung oder einem umfassenden Handlungsplan. Es können auch Reste von Unklarheit zurückbleiben oder im Prozess neue Unklarheiten auftauchen, wie dies beispielsweise im empirischen Modell zur dritten idealtypischen Episode (2017_2119_03\#3) der Fall ist. Hier wird nachvollziehbar, warum im Focusing-Prozess das „Empfangen und Schützen“ (vgl. Kapitel 3.3) als wichtig erachtet wird. Auch im Coaching ist mit einem inneren Kritiker zu rechnen, der als sekundäre Reaktion das Erarbeitete in Frage stellt. Hier scheint regelmäßig empfehlenswert, am Ende des Prozesses auch eine ausreichende Würdigung des Erarbeiteten vorzunehmen. Zweifel und Restunsicherheiten müssen dabei nicht ausgeblendet werden. Wenn es aber gelingt, das Erarbeitete in konkrete Handlungsplanungen einfließen zu lassen, kann der Klient sein Ergebnis auch für weitere Schritte nutzen (Rice \& Saperia, 1984, S. 40).

\subsubsection{Zur Focusing-Methode}

Auf die Methode des Focusing wurde bereits in der Einführung dieser Arbeit ausführlich eingegangen (vgl. Kapitel 3.3), und im Diskurs (vgl. Kapitel 4.3) wurden körperorientierte, achtsamkeitsbasierte, psychoanalytische und hypnosystemische Konzepte besprochen. Ebenso wurden die möglichen Begrenzungen aufgezeigt, die sich beim Einsatz der Focusing-Methode im Coaching ergeben. Deutlich wurde auch, dass experiencingorientiertes Coaching nicht gleichzusetzen ist mit Focusing, und dass Experiencing auch im Kontext unterschiedlicher Methoden gefördert werden kann (Gendlin, 1996, S. 271). Für Coachs, die gezielt mit der Focusing-Methode arbeiten wollen, ergeben sich aus dieser Arbeit Überlegungen, die die 
bisherigen Erkenntnisse zum Einsatz vom Focusing ergänzen können.

Wenn im Coaching Unklarheit bearbeitet werden soll, ist es für Coachees wichtig, die eigene Aufmerksamkeit von der Beschäftigung mit dem Umfeld abzuwenden und dem inneren Erleben zuzuwenden und dort zu verweilen, so dass Neues entstehen kann. Roth (2019, S. 308) weist darauf hin, dass dies am besten in einem Zustand entspannter Aufmerksamkeit gelingt. Die Focusing-Methode bietet mit ihrem Schritt des Raum Schaffens hierfür einen guten Rahmen. Sicher lassen sich auch andere körperorientierte oder achtsamkeitsbasierte Methoden einsetzen, die dem Klienten helfen, in einen solchen Zustand der Entspannung und gleichzeitig der gegenwärtigen Aufmerksamkeit zu kommen. Es kann davon ausgegangen werden, dass ein solches Vorgehen den Klienten darin unterstützt, seinen Bezugspunkt quasi von außen nach innen zu verlagern und damit auch zu einem vertieften Experiencing zu gelangen. Dem Prozessschema folgend, wie es im theoretisch-empirischen Modell abgebildet ist, scheint der Zeitpunkt für diese Aufmerksamkeitsfokussierung auf den Körper und das gegenwärtige Erleben dann günstig zu sein, wenn nach einer ersten Situationsbeschreibung die Trilemma-Problematik erkennbar ist und es nun darum geht, diese weiter zu bearbeiten. Eine zu frühe Hinwendung zu diesem Schritt des Raum Schaffens würde möglicherweise dazu führen, dass der Trilemma-Ausgangspunkt und damit das eigentlich zu bearbeitende Thema zu undeutlich wäre.

Bei den untersuchten Episoden fällt auf, dass im Zusammenhang mit dem Selbsterleben auch Metaphern entwickelt werden, mit denen die Coachees dann weiterarbeiten. Die Methode des Focusing arbeitet hier mit dem „Griff“ für das Körpergefühl, um eine Symbolisierung entstehen zu lassen. Auch wenn der „Griff“ mehr beinhaltet als eine Metapher, bestätigen die empirischen Ergebnisse, dass es für den Klienten von Nutzen ist, wenn er ein Wort oder Symbol als kognitive Übersetzung des Körperempfindens findet. Wenn dieses Wort oder Symbol sich nicht automatisch beim Klienten einstellt, kann der Coach dem Klienten eine entsprechende Hilfestellung auf der Basis der jeweiligen Focusing-Schritte geben.

Abschließend sei darauf hingewiesen, dass auch im Coaching das gilt, was Benecke (2014, S. 643) als ,2-steps-forward, 1-step-back fashion“ beschreibt. Coachs sollten dem Klienten also auch im Focusing die Möglichkeit für Rückkoppelungs- oder Zirkularitätsschleifen einräumen, ohne dabei das Ziel des Felt Shift aus den Augen zu verlieren.

\subsubsection{Methodischer Impuls: Trilemma-Übung}

Es kann davon ausgegangen werden, dass für das experiencingorientierte Arbeiten im Coaching eine große methodische Breite in Betracht kommt. Hinweise dazu ergeben sich aus den in Kapitel 4.4 umrissenen Varianten des Experiencing. Um gezielt auf Trilemma-Situationen einzugehen, können Techniken wie beispielsweise die Arbeit mit dem Inneren Team (Schulz v. Thun, 2014), die Arbeit mit Selbstanteilen (Goldmann, 2014; Mearns, 1999) oder die Arbeit mit Stühlen (Perls, 2018) zum Einsatz kommen. Ergänzend dazu soll in diesem Kapitel ein methodisch-technischer Impuls ausgearbeitet werden, mithilfe dessen Trilemma- 
Situationen im Coaching expliziert und bearbeitet werden können. Der Autor geht dabei von folgenden Vorannahmen aus:

- Im Coaching ist es im Allgemeinen anschlussfähiger, nah am Thema zu arbeiten und eine bestimmte Arbeitseinheit mit einer Situationsbeschreibung einzuleiten statt mit einer Körper- oder Entspannungsübung

- Für die Aktivierung des Selbsterlebens und des Spürens unterschiedlicher Dimensionen kann es für Klienten hilfreich sein, nicht nur am Tisch sitzend zu arbeiten, sondern auch unterschiedliche körperliche Positionen einzunehmen

- Die Aufgabe „Umgehen mit dem Unklaren“ bringt mit sich, dass zu Beginn kein klares Anliegen und auch kein klares Ziel formuliert werden kann, sondern dass lediglich ein Ausgangspunkt, verbunden mit einem gewissen Veränderungswunsch, benannt werden kann. Ein solcher Ausgangspunkt kann sein: Unklarheit, Unbehagen, Belastungserleben, Spannungserleben

- Der erste Teilschritt der Aufgabenbearbeitung besteht im Herausarbeiten der Trilemma-Situation

- Die weitere Bearbeitung erfordert ein Verweilen beim Selbsterleben im Sinn eines dialektischen Umgangs mit unterschiedlichen Erlebensinhalten

- Vorausgesetzt wird eine Grundhaltung des Coachs im Sinn der personzentrierten Beziehungsvariablen.

Die Trilemma-Übung folgt nun den vier Phasen, die im theoretisch-empirischen Veränderungsmodell dargestellt sind: Situationsbeschreibung, Vertiefung der Situationsbeschreibung, Verweilen beim Selbsterleben, Lösungsansatz.

Zunächst ist es Aufgabe des Coach, dem Klienten bei seinen Schilderungen aufmerksam zuzuhören, auf innere Unklarheit oder innere Irritationen zu achten und mögliche Hinweisreize und Indikationen, wie sie in den Kapiteln 7.1, 7.4 und 7.5 dargestellt sind, wahrzunehmen und gemeinsam mit dem Klienten zu klären, ob die entsprechende Unklarheit bearbeitet werden soll. Ist dies gegeben, kann der Coach zu einer Vertiefung der Situationsbeschreibung einladen und dort besonders auf das Herausarbeiten der Trilemma-Situation achten. Dazu hört er dem Klienten zunächst weiter zu und achtet auf Schlüsselworte, die den Trilemma-Komponenten „Problematisches Verhalten des Umfelds, mit Bezug zur eigenen Person“, „Affektiv-kognitive Wahrnehmung: Einerseits...“ und „Affektiv-kognitive Wahrnehmung: Andererseits..." zugeordnet werden könnten. Diese Schlüsselworte notiert er auf Moderationskarten, wobei sich die Karten, die sich auf problematisches Verhalten des Umfelds beziehen, farblich von den anderen Karten unterscheiden sollten.

Im Lauf der Vertiefung der Situationsbeschreibung kann der Coach dann die beschrifteten Karten als Bodenanker auf dem Boden auslegen, und zwar als Dreieck analog zum TrilemmaSchaubild. Nun liegen an jeder Ecke des Dreiecks mehrere Karten, und der Klient kann mit Unterstützung des Coachs klären:

- Treffen die Worte, die auf den Karten stehen, den Sachverhalt? Wie könnte eine 
möglicherweise noch besser zutreffende Formulierung der Worte lauten? Welche Worte / Karten sind zu ergänzen oder auszutauschen?

- Lassen sich für die drei Ecken Zusammenfassungen / Überschriften finden?

- In welchem Abstand sollten sich die drei Ecken zueinander befinden, um möglichst gut abzubilden, was im Klienten vor sich geht?

Der Klient und der Coach arbeiten dann so lange an und mit den Karten und verändern die Stichworte und Positionen, bis der Klient sich möglichst gut wiederfindet in der Abbildung. Der Coach achtet darauf, dass es gelingt, alle drei Komponenten klar zu konturieren, er nimmt aber keinen Einfluss auf den konkreten Inhalt.

Ein weiterer Teilschritt im Übergang von der Vertiefung der Situationsbeschreibung zum Verweilen beim Selbsterleben ist das Herstellen einer gewissen Distanz zur Komponente des Umfeldverhaltens. Hier kann der Coach den Klienten gezielt auffordern, die entsprechenden Karten so im Raum zu platzieren, dass die Distanz zur eigenen Person seinem momentanen Empfinden entspricht. Coach und Klient können dann miteinander überlegen, ob diese Distanz ausreicht, damit der Klient sich den Karten auf der Person-Person-Achse zuwenden kann. Bei Bedarf können sie besprechen, wie die Karten zum Umfeldverhalten in einer größeren Distanz zu den beiden anderen Komponenten platziert werden können. Ist eine ausreichende Distanz hergestellt, kann der Coach den Klienten einladen, innerlich innezuhalten und zu entspannen - gegebenenfalls mit einer entsprechenden kurzen Anleitung. Dann begibt sich der Klient zuerst zu einer der beiden ,affektiv-kognitiven Komponenten“, stellt sich dort bewusst auf, liest die entsprechenden Stichworte und spürt in sich hinein: wo im Körper reagiert etwas? Welche Gefühle entstehen? Welche inneren Impulse entstehen? Nach einer Weile begibt sich der Klient zur anderen affektiv-kognitiven Komponente und wiederholt dort das „Insich-hinein-Spüren“. Dieser Vorgang kann, bezogen auf die beiden affektiv-kognitiven Komponenten des Trilemmas, mehrfach wiederholt werden, und der Klient bringt dabei die unterschiedlichen Stränge seines Selbsterlebens miteinander in Kontakt.

Der Coach achtet auf den Raum zum Verweilen und begleitet den Klienten in dieser Phase mit Hilfe der Gesprächsregeln, wie sie in Kapitel 7.8.3 beschrieben sind. Er vertraut darauf, dass sich aus dem Verweilen beim Selbsterleben quasi automatisch ein Prozess entwickeln wird, der dem Klienten ein besseres Verstehen seiner selbst ermöglicht, zu einer veränderten Deutung, Bewertung oder Haltung führt und mit einer gewissen Klarheit und Erleichterung einher geht. Möglicherweise zeigt sich die Veränderung im Rahmen der Übung auch darin, dass der Klient sich nicht nur zwischen den beiden Bodenankern hin und her bewegt, sondern noch eine dritte Position einnimmt. Solche und andere kreativen Ausgestaltungen sind ausdrücklich willkommen.

Zum Abschluss der Bearbeitung, wenn für den Klienten ein Lösungsansatz erkennbar ist und diese Lösung auch im Körper eine stimmige Resonanz ausgelöst hat, wenden sich Coach und Coachee wieder dem Bodenanker zu, der für das Umfeld steht. Der Coachee kann nun für sich klären, in welcher Nähe / Distanz er sich weiterhin zu dieser Komponente positionieren 
möchte und welche inneren Anteile dabei eine Rolle spielen sollen. Dies kann auch dadurch verdeutlicht werden, dass der Klient die Bodenanker noch abschließend so positioniert und beschriftet, dass sie nicht mehr das anfängliche Trilemma abbilden, sondern die neu gefundene Klarheit.

Nach dem Abschluss der Trilemma-Übung kann dann die veränderte Haltung im Gespräch vertieft werden, und der entsprechende Handlungsansatz kann konkretisiert werden. Im Rahmen der Prozessreflexion achtet der Coach darauf, den Prozess und das Ergebnis zu schützen, wie das auch aus der Focusing-Anleitung (Kapitel 3.3) bekannt ist. 


\section{Methodische Reflexion}

Mit der vorliegenden Studie wurde sowohl in der Forschung als auch für das Coaching ein bis dahin noch wenig erschlossenes Feld bearbeitet. Ziel war, bestehendes Wissen aufzuarbeiten, Vorannahmen kritisch zu reflektieren und auf Basis der empirischen Untersuchung Neues zu entdecken. Dazu wurden Coachingprozesse mit der Methode der qualitativen Task Analyse untersucht. Die Entscheidung für ein qualitatives Vorgehen wurde im Rahmen der Einordnung und der Ziele der Studie (Kapitel 5.1) begründet. Zur konkreten Durchführung der qualitativen Task Analyse fand der Autor nur wenig Literatur, an der er sich orientieren konnte. So soll hier eine methodische Reflexion erfolgen, die sich mit der Anwendung von Gütekriterien in dieser Task Analyse auseinandersetzt, und ebenso mit den Möglichkeiten und Grenzen der Methode. Bei den Gütekriterien orientierte sich der Autor an den in Kapitel 5.5 genannten Aspekten, und hier vor allem an den Forderungen von Breuer et al (2009). Im Interesse einer Gesamtreflexion der methodischen Vorgehensweise soll hier allerdings auf eine Darstellung analog zur dortigen Kriterienliste verzichtet werden, vielmehr sollen die entsprechenden Aspekte jeweils an geeigneter Stelle in den folgenden Abschnitten eingearbeitet werden.

\subsection{Indikationen zur Methodenwahl}

Die Ziele dieser Studie und die Einordnung im Rahmen der Change Process Research wurden in den Kapiteln 5.1 und 5.2 ausführlich beschrieben. Zentrale Aspekte dabei waren:

- Es sollten natürliche Coachingprozesse untersucht werden

- Das Hauptinteresse galt nicht dem Verhalten der Coach und auch nicht der Interaktion, sondern dem intrapersonalen Prozess der Klientin, soweit er im Rahmen des konkreten Coachingprozesses beobachtbar war

- Theoretischer Bezugspunkt für das Forschungsprojekt waren experienzielle Konzepte, wie sie insbesondere im Focusing zum Einsatz kommen

- Ziel war, ein detailliertes Verständnis für experienzielle intrapersonelle Veränderungsprozesse zu entwickeln. Es ging nicht darum, experienzielle Konzepte oder Focusing als Methode zu validieren.

Die Studie folgt damit dem Desiderat von Möller \& Kotte (2011, S. 453) nach Prozessforschung zu der Frage, was im Coaching überhaupt passiert. Allerdings muss konstatiert werden, dass die von den Autorinnen geforderte Vielschichtigkeit der Prozessforschung im Sinn der Einbeziehung von Coach- und Klientinnenperspektive, der Verwendung von Videoanalysen oder der Verwendung von Intersessionsskalen in der Forschungspraxis schnell an Grenzen stoßen. Zum einen lassen sich im Rahmen von Einzelstudien jeweils nur ausgewählte 
Aspekte untersuchen, und zum anderen wurde bereits im Zusammenhang mit der Vorstellung der Kasseler Coachingstudie auf die Zurückhaltung von Coachs gegenüber Forschungsprojekten hingewiesen.

Nachdem die Entscheidung für eine qualitative Vorgehensweise getroffen war, war zu klären, wie die oben genannten zentralen Anliegen am besten umgesetzt werden könnten. Ausgangspunkt dazu war der Anspruch, Erkenntnisse möglichst in Form von natürlichen Beobachtungen zu sammeln (Elliot, 1984, S. 251). Einer teilnehmenden Beobachtung (vgl. Lamnek, 2005, S. 329 und 552) stand dabei entgegen, dass Coaching in aller Regel unter vier Augen stattfindet und Coachs und Coachees zumeist diesen vertrauten Rahmen nicht preisgeben wollen. Auch eine anstelle der teilnehmenden Beobachtung denkbare Videoaufzeichnung von Coachinggesprächen wäre sehr wahrscheinlich sowohl bei Coachs als auch bei Coachees aufgrund der besonderen Intimität des Raums zwischen Coach und Coachee auf Akzeptanzprobleme gestoßen (Gessnitzer et al, 2018, S. 39; Kotte et al, 2015, S. 25). So diente als sinnvolle und praktikable Alternative für diese Untersuchung die Audioaufzeichnung und anschlieBende Transkription von Coachinggesprächen, um die verbalen Inhalte der Gespräche darzustellen und inhaltlich zu analysieren. Damit lehnte sich das Vorgehen an die Erfahrungen der psychologischen Prozessforschung an, wo die Audioaufzeichnung neben der fragebogenbasierten Herangehensweise als grundlegende Methode etabliert ist (vgl. Benecke, 2014, S. $627)$.

Bei der Überlegung, auf welchem Weg die Audiographien bzw. Transkripte der Coachinggespräche ausgewertet werden sollten, erwog der Autor zunächst eine Untersuchung im Rahmen der qualitativen Inhaltsanalyse (Mayring, 2007) oder der Reflexive Grounded Theory Methodologie (Breuer et al, 2009). Letzten Endes wurde dann die Entscheidung zugunsten der qualitativen Task Analyse getroffen, da sie Ähnlichkeiten mit diesen beiden Methoden aufweist, ihnen gegenüber aber Besonderheiten und Vorteile aufweist, die als entscheidend erachtet wurden und sich in der Rückschau auch bestätigen:

- Die Task Analyse ermöglicht, von Theorie auszugehen und ein vorhandenes theoretisches Modell mit dem empirischen Befund abzugleichen und damit weiterzuentwickeln. Das abduktive Vorgehen im Rahmen der Task Analyse entsprach dem Anliegen der Studie

- Der Fokus der Untersuchung sollte möglichst uneingeschränkt auf dem klientinnenseitigen Verhalten bzw. Veränderungsprozess liegen. Dies sollte keine Ignoranz gegenüber der Relevanz des Interaktionsverhaltens zwischen Coach und Coachee darstellen, sondern eine Konzentration auf den intrapersonalen Prozess der Coachee ermöglichen. Der in der Task Analyse vorgesehene Weg, diesen Prozess anhand der Rekonstruktion eines psychologischen Aufgabenmodells differenziert zu beschreiben, erwies sich als gut umsetzbar und gewinnbringend. Wenn der Fokus einer Untersuchung auf dem klientinnenseitigen Prozess liegen soll, kann die Task Analyse als geeignete Analysemethode bestätigt werden 
- Ein Problem der Change Process Research ist die Eingrenzung der Untersuchung auf diejenigen Episoden, die auch eine gewisse Ergebnisrelevanz haben (vgl. Kapitel 5.2). Die taskanalytische Vorgehensweise schlägt hierzu eine mehrschrittige Samplingstrategie vor, die sowohl die Bearbeitung einer psychologischen Aufgabe als auch das Ergebnis als unmittelbar zu beobachtende Veränderung berücksichtigt und damit gut geeignet scheint, auch tatsächlich relevante Prozesse zu identifizieren.

\subsection{Theorie- und Praxisorientierung in der Task Analyse}

Möller \& Kotte (2011, S. 425) erachten die Verschränkung von Expertinnenwissen und Wissenschaftssystem als sinnvolles Ziel der Coachingforschung. Gemeint ist damit eine enge $\mathrm{Zu}-$ sammenarbeit zwischen theoretisch arbeitenden Forscherinnen und praktizierenden Coachs, die im deutschsprachigen Kontext teilweise kontrovers diskutiert wird (ebd.). Die Task Analyse schlägt hier einen eigenen Weg der Verschränkung von Praxis und Wissenschaft ein, indem sie regelmäßig „clinical experience“ (Greenberg, 2007, S. 16) bei der Durchführung der empirischen Analyse voraussetzt.

Der Autor dieser Studie nimmt aufgrund seines personzentriert-experienziellen Ausbildungsstands und aufgrund seiner Praxis als freiberuflich tätiger Coach für sich in Anspruch, hinreichend beratungswissenschaftlich geschult zu sein, um sowohl spezifisches Theoriewissen als auch Praxiserfahrung in den Auswertungsprozess einfließen zu lassen.

Auf die in diesem Zusammenhang auftauchende Frage der Neutralität bzw. Objektivität der Forschung wurde bereits in Kapitel 5.5 eingegangen, und zur Absicherung der Neutralität wurde im Rahmen der Task Analyse die eigene kognitive Forschungslandkarte (vgl. Kapitel 6.5) offengelegt. Auch die ausführliche Erörterung der theoretischen Grundlagen zum Experiencing dient der Darlegung der Sichtweisen des Forschenden, um die Einflussnahme impliziter Sichtweisen und Interessen auszuschließen.

In Kapitel 6.8.5 wurde im Rahmen der theoretischen Rückbindung des empirischen Modells beschrieben, dass im Verlauf der Untersuchung eine stringente Differenzierung zwischen den Begriffen Gefühl, Emotion und Affekt und auch zwischen dem Körpererleben, Körperspüren und Körpergefühl schwer umzusetzen war. Dies wurde dort bereits theoretisch eingeordnet. Bei der methodischen Reflexion kann in der Rückschau festgestellt werden, dass dieser Problematik durch einen weniger praxisorientierten und mehr theorieorientierten Fokus bei der Bildung der Makrokategorien hätte Rechnung getragen werden können. Beispielsweise wäre es möglich gewesen, statt der an der Coachingpraxis orientierten Makrokategorien Selbsterleben und Kontextbezug eher theoriebezogene Makrokategorien wie Emotion, Affekt, Kognition oder auch Makrokategorien nach dem Vier-Ebenen-Modell der Persönlichkeit nach Roth \& Ryba (2016, vgl. Kapitel 4.2) zu bilden. Damit wäre aber der in der Fragestellung 
enthaltene Praxisbezug im Sinn des Anliegens, Zusammenhänge und Muster herauszuarbeiten, wann ein experiencingorientiertes Vorgehen im Coaching besonders hilfreich erscheint und welche Ausstiegsmomente aus den Veränderungsprozessen erkennbar werden, in den Hintergrund gerückt.

Hier zeigt sich ein Dilemma, das bei der Verschränkung von Forschung und Praxis auftreten kann: Grundlagenforschung und Praxisforschung verfolgen zwei Interessensstränge, die sich mitunter schwer in einer gemeinsamen Forschungsfrage oder einem eng umrissenen Forschungsprojekt vereinen lassen. Auch wenn die Task Analyse per se nicht auf eine Grundlagenorientierung oder Praxisorientierung festgelegt ist, war in diesem Fall spätestens bei der Bildung der Metakategorien eine Entscheidung zu treffen, ob eher psychologische Grundlagen von Interesse sind oder ob eher die Nähe zur Coachingpraxis im Vordergrund stehen soll. Die Entscheidung für die Nähe zur Coachingpraxis traf der Autor zu diesem Zeitpunkt eher intuitiv bzw. auf der Grundlage der Themenstellung. Rückblickend kann festgestellt werden, dass durch das Bilden der Makrokategorien eine gewisse Weichenstellung in Richtung Grundlagenorientierung oder Praxisorientierung vorgenommen wird, und dass es hilfreich für Forschende ist, sich dessen bewusst zu sein.

\section{3 Übertragbarkeit der Studie}

Der Autor geht davon aus, dass die Ergebnisse und Schlussfolgerungen dieser Studie auch auf Kontexte außerhalb der Studie übertragbar sind. Um den Leserinnen hierzu eine eigene Einschätzung zu ermöglichen, wurde bei der Durchführung der Studie eine genaue Beschreibung des Kontexts der Kasseler Coachingstudie gegeben. Die Art und Weise, wie für die Teilnahme an der Studie geworben wurde, stellte sicher, dass nur erfahrene und in den entsprechenden Berufsverbänden beheimatete Coachs an der Studie teilnahmen. Dadurch, dass die Coachingprozesse größtenteils vollständig audiographiert sind und die Audiographien vollständig angehört wurden, konnte eine weitere Überprüfung und Vorselektion stattfinden, um in die Studie tatsächlich nur solche Coachinggespräche einzubeziehen, die der hier zugrunde gelegten Definition von professionell durchgeführtem Coaching mit arbeitsweltlichem Bezug genügen.

Kritisiert werden könnte, dass die Coachs zwar die eigentlich Teilnehmenden an der Studie waren, dass der Autor aber darauf verzichtete, detaillierte Daten über sie zu erheben bzw. in die Studie einfließen zu lassen. Zwar wäre dies unter Hinzuziehung der entsprechende CoachFragebögen, die im Rahmen der Kasseler Coachingstudie erhoben wurden, möglich gewesen. Der Autor sah darin aber keinen Mehrwert hinsichtlich des Erkenntnisgewinns zur Fragestellung. Relevant aus Sicht des Autors schien lediglich, dass die coachseitigen Rahmenbedingungen für alle untersuchten Gespräche insofern identisch waren, als dass es sich in allen Prozessen um externe, freiberuflich tätige Coachs mit einer spezifischen Coachingausbildung und mit Berufserfahrung als Coachs handelte. Die Tatsache, dass nicht auf eine schulenspe- 
zifische Ausrichtung geachtet wurde und bezüglich der Coachs keine weiteren Auswahlkriterien hinzugezogen wurden, spricht aus der Sicht des Autors dafür, dass die Ergebnisse der Studie auf andere Coachingprozesse übertragen werden können.

Um die intersubjektive Nachvollziehbarkeit einer Studie zu gewährleisten, wird in der Grounded Theory Methodoloy in der Regel eine Diskussion und Interpretation der Ergebnisse in Gruppen gefordert. Der Autor zog diese Möglichkeit zunächst in Erwägung, distanzierte sich dann aber wieder davon. Im Rahmen der Task Analyse schien es wenig hilfreich, nur Codes oder Modelle zu diskutieren, solange die Diskussionsgruppe nicht in den gesamten Herleitungsprozess dieser Zwischenergebnisse einbezogen war. Der Autor ging davon aus, dass bei einer abduktiven Vorgehensweise, wie sie hier zum Einsatz kam, bereits die Phase der theorieorientierten Modellbildung relevant für die Erkenntnisse und Ergebnisse ist, so dass die Gruppendiskussion für jede einzelne Etappe des Forschungsprozesses erforderlich gewesen wäre. Dies aber hätte den Rahmen dieser Arbeit überfordert, es wäre nur im Rahmen einer von Beginn an in einem Forschendenteam gemeinsam durchgeführten Studie umsetzbar gewesen. Gleichwohl ist anzuerkennen, dass der Verzicht auf die Interpretation der Ergebnisse im Rahmen einer Diskussion mit außenstehenden Fachkolleginnen auch eine Limitation dieser Arbeit darstellt.

\subsection{Vertrauenswürdigkeit und Zuverlässigkeit der Studie}

Ein weiterer Aspekt der Nachvollziehbarkeit einer Studie, und damit ihrer Vertrauenswürdigkeit, ist die Plausibilität der Interpretationen. Um den Weg von der Datenbasis hin zu den Interpretationen möglichst gut nachvollziehbar zu machen, wurde das Anliegen der Studie ausführlich beschrieben (Kapitel 1.1 und Kapitel 5.1), und die Methode der Task Analyse als systematisches und regelgeleitetes Verfahren wurde vor der Durchführung in einem eigenen Kapitel (Kapitel 5.3) umfassend dargestellt. In einem mehrstufigen und vollständig dokumentierten Prozess wurde besonderer Wert auf die Auswahl der auszuwertenden Coachinggespräche gelegt. Die Transkriptionsregeln und die Sampling-Strategie wurden dargelegt. Die weitere Auswertung wurde Schritt für Schritt detailliert beschrieben. Es wurde Wert gelegt auf eine ausführliche Beschreibung der Daten und der Forschungsschritte und Erkenntnisse, um den Forschungsprozess jederzeit transparent und überprüfbar zu gestalten. Ergebnisse wurden unter Bezugnahme auf das Datenmaterial dargestellt und mit Original-Zitaten belegt.

Ergänzend wurde die Re-Rating-Reliabilität der Experiencing-Stufen anhand der Anleitung zur Experiencing-Skala überprüft. Die erste Einschätzung zum Experiencing erfolgte bei den idealtypischen Prozessen im März 2020 und bei den kontrastierenden Prozessen Anfang April 2020. Ende April 2020 wurde dann eine Wiederholung des Ratings vorgenommen, ohne Kenntnis der Details des ersten Ratings. Eingeschätzt wurde der Modus-Wert der zu analysierenden Gesprächseinheiten jeweils zur zehnten Minute (sh. Anlage 62: Re-Rating und Berechnung der Re-Rating-Reliabilität). 
Zwischen den beiden Rating-Zeitpunkten wurde der Korrelationswert Cohen's Kappa berechnet (vgl. Döring \& Bortz, 2016, S. 568), um die Übereinstimmung des Rating zwischen den beiden Zeitpunkten einordnen zu können. Es wurde ein Kappa von $\kappa=0.70$ berechnet, und für das gewichtete Kappa wurde ein Wert von $\kappa=0.82$ berechnet. Nach Landis \& Koch (1977) sind Kappa-Werte $a b \kappa=0,60$ als substanziell, und $a b \kappa=0.80$ als fast perfekt zu interpretieren. Eine vollständige Übereinstimmung der Werte ergäbe sich bei $\kappa=1$.

Bei der Durchführung der Studie hat der Autor auf eine methodische Triangulation, wie Lincoln \& Guba (1985) sie fordern, verzichtet. Dies geschah vor dem Hintergrund, dass aus konstruktivistischer Sicht nicht sichergestellt ist, dass unterschiedliche Methoden auch dieselben Phänomene untersuchen, so dass nicht automatisch davon ausgegangen werden kann, dass eine solche Triangulation tatsächlich der Validierung des Ergebnisses dient (Lamnek, 2005, S. 160). Anzumerken ist jedoch, dass die Task Analyse selbst mehrere und sich ergänzende methodische Schritte integriert, so dass hier bereits unterschiedliche Perspektiven einfließen (Experiencing-Rating, Codierung anhand des theoretischen Modells, empirische Codierung). Auch vor diesem Hintergrund wurde eine weitere Auswertungsmethode nicht in Erwägung gezogen.

\subsection{Methodische Limitierung}

Auch wenn beim Sampling auf Basis einer vergleichsweise großen Grundgesamtheit (in der Studie als relevante Stichprobe bezeichnet) Wert darauf gelegt wurde, der Vielfältigkeit der Erfahrungen Raum zu geben und möglichst unterschiedliche Fälle in die Untersuchung einzubeziehen, erhebt eine qualitative Studie wie diese nicht den Anspruch, alle im Feld vorkommenden Muster zu erfassen. Insofern ist nicht auszuschließen, dass durch das Hinzuziehen weiterer idealtypischer Beispiele eine weitere Ausdifferenzierung des empirisch-theoretischen Modells möglich gewesen wäre, und dass durch das Hinzuziehen weiterer kontrastierender Beispiele weitere Ausstiegsmomente aus dem Prozess sichtbar geworden wären. Gleichzeitig ist in der Rückschau festzustellen, dass die unterschiedlichen Prozesse auch unterschiedliche Spezifitäten aufzeigten, so dass der Autor davon ausgeht, dass bereits eine gewisse Vielfalt der Phänomene erfasst wurde. Hinzu kommt, dass sich im Samplingprozess zeigte, dass trotz einer anfänglich umfangreich wirkenden Gesamtstichprobe nur eine begrenzte Anzahl idealtypischer Prozesse identifiziert werden konnte (sh. Kapitel 6.8.1 bzw. Anlage 53: Sample-Bildung). Das kann einerseits so gedeutet werden, dass eine weitere Ausdifferenzierung anhand weiterer Beispiele nicht notwendig war. Andererseits bleibt offen, inwieweit in der Gesamtstichprobe weitere intrapersonale Veränderungsprozesse, die nicht im Kontext von Experiencing stehen, stattgefunden haben und wie genau diese vonstattengingen. Hier könnten weitere Forschungsvorhaben ansetzen.

Eine ebenso zu berücksichtigende Limitation der Studie ergibt sich aus der Entscheidung, bei der Fallauswahl auf eine Selektion hinsichtlich des schulen- oder methodenorientierten Vorgehens zu verzichten. Es zeigte sich, dass aus der großen Grundgesamtheit letzten Endes nur 
eine begrenzte Anzahl von für die Fragestellung relevanten (Sub-) Prozessen zur Verfügung stand, weil der Felt Sense bzw. das Experiencing in zahlreichen Prozessen keine erkennbare Rolle spielte. Hier wäre sicher auch ein Vergleich mit solchen Prozessen interessant gewesen, bei denen die Coachs explizit focusingorientiert oder experiencingorientiert vorgehen. So hätte die in der Focusing-Community gestellte Frage „What does Focusing add to Coaching?“ (vgl. Kapitel 1.1) sicher präziser beantwortet werden können. Allerdings wäre durch die Hinzunahme von methodenspezifischen Kriterien der Fokus von der Beschreibung des intrapersonalen Veränderungsprozesses abgerückt.

Die aus Sicht des Autors größte Limitierung der Arbeit besteht darin, dass eine umfassende Task Analyse nach der qualitativ orientierten Discovery-Phase noch eine quantitative Validation erfordert.

„Thus, the qualitative investigation is not consolidated until a validation study is done, usually in the form of a second, quantitative study. It stands to reason that two phases of investigation, each requiring a different data set, make for a lengthier inquiry than a single qualitative inquiry." (Pascual-Leone et al, 2009, S. 530)

So bedürfen die Erkenntnisse dieser Arbeit in einem zweiten Schritt und auf Grundlage einer weiteren Erhebung von Coachingprozessen einer weiteren Bestätigung. 


\section{Fazit und Ausblick}

\subsection{Veränderungskonzept}

Benecke (2014, S. 33, vgl. Kapitel 2.3) benennt vier Bestandteile von Veränderungskonzepten anhand derer hier ein Resümee zu den Erkenntnissen dieser Studie gezogen werden soll:

1. Annahmen über Wirkprozesse:

Das hier zugrunde gelegte Konzept der Veränderung basiert auf experienziellen Theorien und den dort beschriebenen affektiv-kognitiven Mechanismen. Diese sind insbesondere von Sachse et al (1992) schlüssig dargestellt. Gleichwohl ergibt sich hinsichtlich des Felt Sense, wie Gendlin (1996) ihn konzeptualisiert hat, eine gewisse theoriebezogene Problematik, die durch die Studie nicht ausgeräumt, sondern eher bestätigt wurde. Wird der Felt Sense nicht als eigenständiges psychologisches Konstrukt betrachtet, sondern eher im Sinne von Sachse et al (1992) als kognitive Repräsentation affektiver Bedeutungen, können die Annahmen des experienziellen Konzepts über Wirkprozesse der Veränderung im Wesentlichen auch im Coaching zugrunde gelegt werden. Im Rahmen der Modellerarbeitung konnte diese Studie die entsprechenden Prozesse im Coaching anschaulich darstellen.

2. Veränderungsstrategien:

Als Ergebnis der Task Analyse wurden sowohl intrapersonale Bedingungen der Veränderung beim Coachee beschrieben als auch äußere, auf die Interaktion zwischen Coach und Coachee bezogene Bedingungen, und es wurde beschrieben, welche Haltung und welche Verhaltensweisen aufseiten des Coach notwendig sind, um die Veränderung zu fördern. Mit dem empirisch-theoretischen Modell wurde ein spezifisch auf Coachingprozesse zurückzuführendes Ablaufmodell entwickelt, das unterschiedliche Phasen zum Veränderungsverlauf und die dort stattfindenden Mikroprozesse spezifiziert.

3. Methoden:

Beispiele für konkrete Interventionen und Techniken, mittels derer die Veränderung gefördert werden kann, wurden genannt und veranschaulicht. Dass dies nur exemplarisch geschah, könnte aus Sicht der Coachingpraktiker als Mangel betrachtet werden. Allerdings war es auch nicht Intention dieser Studie, ein Methodenkompendium zu erstellen, sondern vielmehr den Prozess der Veränderung an sich detailliert in den Blick zu nehmen.

4. Wirkungen:

Der Prozess von der Problemindikation hin zur Veränderung wurde beschrieben. 
Es wurde ein Weg aufgezeigt, wie Coachs und Klienten mit Unklarheit oder noch nicht klar beschreibbaren Problemsituationen umgehen können. Als Besonderheit wurde die Trilemma-Situation als Ausgangspunkt herausgearbeitet, und ebenso die Sichtweise, dass im Rahmen dieses Veränderungskonzepts der vorwärtstragende Prozess eine Richtung anzeigt, das konkrete Ziel aber nicht a priori festgelegt werden kann.

So kann als Fazit gesehen werden, dass das experienzielle Veränderungskonzept auf das Coaching angewendet und die dortigen Spezifika herausgearbeitet wurden. Dabei wurde ein Theorie-Praxis-Bezug hergestellt und eine Basis beschrieben, um Veränderungsprozesse im Rahmen von Coaching gezielt und unter Einbeziehung affektiver und kognitiver Aspekte und insbesondere unter Einbeziehung körperbezogenen Erlebens zu fördern.

\subsection{Prozessreflexion}

Ein Forschungsprozess wie dieser ähnelt in gewisser Weise dem Veränderungsprozess im Coaching, wie er im theoretisch-empirischen Modell dargestellt ist. Am Ausgangspunkt dieser Arbeit stand zunächst die diffuse Vorstellung, dass das Felt Sense Konzept im Coaching umfassender nutzbar sein könnte als das bisher sowohl in der Theorie als auch in der Praxis erkennbar war. Damit war für den Autor klar, dass er Coachingprozesse untersuchen wollte, und dass das Felt Sense Konzept dabei auch eine Rolle spielen sollte.

Bei den weiteren Überlegungen zur Zielsetzung und Ausgestaltung der Studie ergaben sich dann zwei Komponenten, die miteinander in Verbindung zu bringen waren. Zum einen wollte der Autor der Frage nachgehen, welche Rolle der Felt Sense im Coaching spielt. Das würde bedeuten, die Focusing-Methode oder zumindest experienzielle Theoriemodelle in die Untersuchung einzubeziehen. Zum anderen interessierte sich der Autor auch für Veränderungsgeschehen im Allgemeinen, und zwar unabhängig von einem eventuellen experiencingorientierten Vorgehen. Das würde bedeuten, sich bei der Auswahl der zu untersuchenden Prozesse nicht an einer bestimmten Schule oder einem bestimmten methodischen Vorgehen zu orientieren.

Zunächst war offen, wie diese beiden unterschiedlichen und in Teilen gegensätzlichen Interessen in einer Studie miteinander verbunden werden könnten. Hinzu kam eine gewisse Skepsis des Autors, ob zur Forschungsfrage überhaupt Neues zu entdecken wäre, weil zu den beiden Aspekten Experiencing und Veränderung im Coaching jeweils schon zahlreiche Ausarbeitungen existierten. Gleichzeitig fand der Autor nur wenige Veröffentlichungen, die Experiencingkonzepte und Veränderungsmomente im Coaching theoretisch fundiert miteinander verbanden.

So war im ersten Schritt eine vertiefende Auseinandersetzung mit Coaching, Veränderung, Experiencing, körperbezogenen Aspekten der Forschung und anderen nahestehenden Konzepten erforderlich, um das Themenfeld zu sondieren und anschließend die am Anfang zwar 
formulierte, aber noch nicht im Detail klare Forschungsfrage zu konkretisieren.

Hinzu kam, dass der Autor sich beim methodischen Vorgehen der Studie nicht an anderen gängigen Verfahren orientieren konnte und wollte, sondern einen spezifischen Weg suchte, um Experiencing und Veränderung miteinander zu verbinden. Die Suche nach diesem Weg war an einigen Stellen mühsam. Eine Lösung zeichnete sich ab, als der Autor sich vom vollständigen Prozess der Task Analyse verabschiedete und entschied, sich auf den entdeckenden, qualitativen Teil der Analyse zu beschränken. Diese Entscheidung fiel nicht leicht, weil dem Autor bewusst war, dass seine Studie damit ohne Ergebnisvalidation abgeschlossen werden würde, und weil er auch kaum auf methodische Vergleiche mit anderen qualitativen taskanalytischen Untersuchungen zurückgreifen konnte.

Als die Entscheidung für diesen Weg trotz der Bedenken getroffen war, eröffnete sich eine Perspektive, die es ermöglichte, Experiencing und Veränderung miteinander zu verbinden und daraus Prozessbeschreibungen abzuleiten, die neue Aspekte zum Veränderungsgeschehen hervorbrachten. Als besonders erhellend und hilfreich erwies sich dabei die Besonderheit der Task Analyse, den zu untersuchenden Prozess mit einer psychologischen Aufgabe zu verbinden und dann anhand der Aufgabenerledigung zu explorieren. Dieses Vorgehen ermöglichte, nicht nur Prozessvariablen zu beschreiben, sondern auch erfolgskritische Aspekte herauszuarbeiten und damit sowohl den Prozess als auch das Ziel in den Blick zu nehmen. Das war für den Autor nicht nur in wissenschaftlicher Hinsicht eine Horizonterweiterung, es wird ihn auch in seiner Coachingpraxis prägen, indem er sich mehr als zuvor die Frage nach der psychologischen Aufgabe stellen wird, die der Coachee in der jeweiligen Gesprächsepisode zu bewältigen hat.

Eine weitere Parallele zwischen diesem Forschungsprozess und einem experiencingorientierten Veränderungsprozess erlebte der Autor beim Umgang mit Komplexität. Die intensive Auseinandersetzung mit Konzepten und das detaillierte Studium der Task-Prozesse anhand des empirischen Materials führte zunächst zu einem immensen Zuwachs an Komplexität. Davon zeugen nicht zuletzt die zahlreichen und umfangreichen Anlagen, auch das empirischtheoretische Veränderungsmodell stellt sich im Vergleich zur anfänglichen Skizze des theoretischen Veränderungsmodells deutlich komplexer dar. Auch wenn in dieser Arbeit die einzelnen Forschungsschritte und Kapitel als aufeinander aufbauend dargestellt sind, bestand die Praxis des Forschens und Schreibens doch mehr in einem zwei-Schritte-vorwärts, ein-Schrittzurück-Prozess, mehr in einem sich spiralförmig oder zirkulär entwickelnden Fortschreiten als in einem linearen Erkenntniszuwachs.

Auch zum Erkenntnisziel war von Anfang an zwar die Richtung klar, nicht aber die spezifische Schwerpunktsetzung, die sich daraus ergeben würde. Dass am Ende die Trilemma-Situation den Ausgangspunkt für Veränderungsprozesse bilden würde, war so wenig absehbar wie überhaupt die Tatsache, dass der Ausgangspunkt des Prozesses eine solch zentrale Rolle spielen würde.

Überraschend und auch erleichternd erlebte der Autor dann gegen Ende seiner Studie eine 
gewisse Komplexitätsreduzierung. Ein Beispiel dafür ist die im Modell enthaltene Distanzierung zum Umfeld und das Verweilen beim Selbsterleben. Dass eine Distanzierung zum Umfeld sinnvoll ist, um sich dem Selbsterleben zuwenden zu können, und dass dann auch eine Zeit des Verweilens beim Selbsterleben notwendig ist, um im Prozess der Selbstklärung voranzukommen, klingt für den Autor am Ende dieser Arbeit wenig komplex, wenn nicht gar einfach und schlicht. Gleichzeitig ist es eine Erkenntnis, die er zum Beginn der Studie und ohne die zwischenzeitliche Erhöhung der Komplexität nicht in dieser Klarheit hätte formulieren können und die er vor allem auch nicht fundiert hätte begründen können.

Eine weitere Erfahrung des Forschungswegs ist die, dass das „Kreuzen“, wie Gendlin (2015, S. 130, vgl. Kapitel 3) es nennt, zu einem unerwarteten Erkenntnisgewinn beitragen kann. In diesem Fall sind sich mit der Neurowissenschaft, der Psychologie, der Beratungswissenschaft und der Philosophie auch unterschiedliche fachliche Disziplinen begegnet, und bei aller Begrenzung des gegenseitigen Verständnisses, die durch unterschiedlich epistemologische Rahmungen entstehen, konnte doch ein gegenseitiges Befruchten stattfinden.

Auch das abduktive Vorgehen war in gewisser Weise ein „Kreuzen“: es knüpfte an bestehende Theorie an und brachte die Theorie in Verbindung mit der Praxis, um so die Theorie weiterzuentwickeln und dann wiederum theoriebasierte Implikationen für die Praxis abzuleiten. So konnte ein empirisch-theoretisches Modell entwickelt werden, das eine Rekonstruktion des intraindividuellen Veränderungsprozess ermöglicht und Rückschlüsse auf experienzielle Prozesse im Coaching zulässt. Die daraus abgeleiteten Implikationen zur Förderung intrapersonaler Veränderungsprozesse und insbesondere die Konkretisierung der TrilemmaSituation im Sinn einer Inkongruenz bzw. Spannung zwischen Situationsbezug und kontradiktorischen Anteilen der affektiv-kognitiven Wahrnehmung bzw. des Selbsterlebens scheinen geeignet, auch über den Rahmen dieser Arbeit hinaus einen Beitrag zur Weiterentwicklung des Coaching in Theorie und Praxis zu leisten.

\subsection{Ausblick}

Ein persönlicher Ausblick wurde bereits im vorhergehenden Abschnitt deutlich: Die intensive Beschäftigung mit dem Experiencing- Konzept und die Task Analyse haben die Sicht des Autors auf Coachingprozesse verändert, so dass er in seiner eigenen Praxis dem Selbsterleben und auch der vom Coachee zu erledigenden Task mehr Aufmerksamkeit widmen wird.

Für die bereits angesprochene Weiterentwicklung des Coaching in Theorie und Praxis ergeben sich aus Sicht des Autors am Ende dieser Studie Aspekte, die zu vertiefen sich lohnen würde. Drei Facetten sollen besonders hervorgehoben werden:

1. Mit der vorliegenden Studie wurde der intrapersonale Veränderungsprozess vor dem Hintergrund von Experiencing untersucht. Interessant wäre, auch andere theoretische Konzeptionen zugrunde zu legen und aus diesen weitere theoretisch-empirische Veränderungsmodelle zu entwickeln. So ließen sich sicherlich Aspekte 
entdecken, die aufgrund des hier zugrunde gelegten Mentalen Modells nicht berücksichtigt wurden, aber doch ergänzende Erkenntnisse zu Veränderungsprozessen beitragen könnten. Der taskanalytische Zugang scheint gut geeignet zu sein für die Entwicklung solcher Modelle.

2. Für eine Validierung des hier erarbeiteten Modells wird es notwendig sein, den zweiten Teil der Task Analyse, eine quantitative Untersuchung auf Basis der hier erarbeiteten Aspekte durchzuführen. So könnten auch die Aussagen zum Körperbezug im Coaching konkretisiert werden, und es könnte geklärt werden, inwieweit Veränderung ohne Experiencing überhaupt stattfinden kann.

3. Die Ausführungen zum Coachverhalten und zum Methodengebrauch in Kapitel 7.8 sind zwar aus den Erkenntnissen der Task Analyse abgeleitet, beruhen aber in Teilen auch auf dem Erfahrungswissen des Autors. Hier wäre künftig eine noch bessere Verschränkung von Theorie und Praxis wünschenswert, so dass Coachingtechniken noch besser wissenschaftlich begründet werden können. 


\section{Literatur}

Ajdukovic, M., Cajvert, L., Judy, M., Knopf, W., Kuhn, H. \& Madai, K. (2014). ECVision. Ein Europäisches Glossar für Supervision und Coaching. Wien: Die Wiener Volkshochschulen

Antal, Z. (2012). Eine empirische Prozess-Outcome-Untersuchung zur Wirksamkeit und Wirkungsweise psychoanalytischer Langzeitpsychotherapien. München: Dissertation zur Erlangung des Doktorgrades der Philosophie an der Ludwig-Maximilians-Universität

Aspland, H. (2008). Alliance ruptures and rupture resolution in cognitive-behavior therapy: A preliminary task analysis. Psychotherapy Research 18 (6), 699-710. DOI: $10.1080 / 10503300802291463$

Athanasopoulou, A. \& Dopson, S. (2018). A systematic review of executive coaching outcomes: Is it the journey or the destination that matters the most? The Leadership Quarterly $29,70-88$

Auckenthaler, A. \& Helle, M. (2002). Gefühle in der Gesprächspsychotherapie. Psychotherapie im Dialog 3(2), 148-152

Auszra, L., Herrmann, I. \& Greenberg, L. (2017). Emotionsfokussierte Therapie. Ein Praxismanual. Göttingen: Hogrefe

Baer, U. \& Frick-Baer, G. (2001): Leibbewegungen: Methoden und Modelle der Tanz- und Bewegungstherapie. Neukirchen-Vluyn: Affenkönig-Verlag

Barnhofer, T. \& Williams, M. (2013). Exkurs: MBCT - aktuelle Befunde und Ausblick. In: T. Heidenreich \& J. Michalak (Hrsg.). Die „dritte Welle“ der Verhaltenstherapie. Grundlagen und Praxis (S. 139-149). Weinheim: Beltz

Bastine, R., Fiedler, P. \& Kommer, D. (1989). Was ist therapeutisch an der Psychotherapie? Versuch einer Bestandsaufnahme und Systematisierung der Psychotherapeutischen Prozessforschung. Zeitschrift für Klinische Psychologie, 18(1), 3-22

Beeman, M. \& Kounios, J. (2015). Das Aha-Erlebnis: wie plötzliche Einsichten entstehen und wie wir sie erfolgreich nutzen. München: Deutsche Verlagsanstalt

Benecke, C. (2014). Klinische Psychologie und Psychotherapie. Ein integratives Lehrbuch. Stuttgart: Kohlhammer

Biermann-Ratjen, E. \& Eckert, J. (2017): Gesprächspsychotherapie. Ursprung - Vorgehen - Wirksamkeit. Stuttgart: Kohlhammer

Böning, U. \& Kegel, C. (2015). Ergebnisse der Coaching-Forschung. Aktuelle Studien ausgewertet für die Coaching-Praxis. Berlin, Heidelberg: Springer 
Bowlby, J. (1988): A secure base. Clinical applications of attachment theory. London: Routledge

Boyesen, G. (1987). Über den Körper die Seele heilen: biodynamische Psychologie und Psychotherapie; eine Einführung. München: Kösel

Breuer, F., Dieris, B. \& Lettau, A. (2009). Reflexive Grounded Theory: eine Einführung für die Forschungspraxis. Wiesbaden: VS Verlag für Sozialwissenschaften

Caspar, F. \& Jacobi, F. (2004): Psychotherapieforschung. In W. Hiller \& E. Leibning (Hrsg.): Lehrbuch der Psychotherapie Band 1 (S. 395-410). München: CIP-Medien

Cooper, M., Watson, J. \& Hölldampf, D. (2010). Person-Centered and Experiential Therapies Work: A Review of the Research on Counseling, Psychotherapy, and Related Practices. Ross-on-Wye: PCCS Books

Creswell, J. \& Plano Clark V. (2011). Designing and Conducting Mixed Methods Research. $2^{\text {nd }}$ edition. Thousand Oaks: Sage Publications

Czogalik, D. (1999). Wege zu effektiven Psychotherapien. In: H. Petzold \& M. Märtens (Hrsg.): Psychotherapieforschung und Praxis. Band 1: Modelle, Konzepte, Settings. (S. 151174). Opladen: Leske \& Buchdrich

Czogalik, D. \& Enke, H. (1997). Allgemeine und spezielle Wirkfaktoren in der Psychotherapie. In: Heigl-Evers, A.; Heigl, F.; Ott, J.; Rüger, U. (Hrsg.): Lehrbuch der Psychotherapie (S. 511-522). Stuttgart: Gustav-Fischer Verlag

Dahlhoff, H. \& Bommert, H. (1978). Forschungs- und Trainingsmanual zur deutschen Fassung der Experiencing-Skala. In H. Bommert \& H. Dahlhoff (Hrsg.): Das Selbsterleben (Experiencing) in der Psychotherapie (S. 63-128). München: Urban und Schwarzenberg

Damasio, A. (1994). Descartes Irrtum. Fühlen, Denken und das menschliche Gehirn. München: List

De Haan, E. \& Nieß, C. (2012). Critical Moments in a coaching case study: illustration of a process research model. Consulting Psychology Journal: Practice and Research 64(3), 198224

De Haan, E. \& Nieß, C. (2018). Kritische Momente im Coaching. Wie Klienten und Klientinnen, Coaches und Interessensgruppen Veränderungen wahrnehmen. In R. Wegener, A. Fritze, M. Hänseler \& M. Loebbert (Hrsg.): Coaching-Prozessforschung. Forschung und Praxis im Dialog. (S. 56-66). Göttingen: Vandenhoeck \& Ruprecht

De Haan, E. \& Mannhardt, S. (2014). Wirkungsvolles Executive-Coaching. Relative Wirkfaktoren effektiven Executive-Coachings: Ergebnisse der "Greatest Ever Executive Coaching Outcome Study. Coaching-Magazin 4/2014. Online unter https://www.coaching-magazin.de/beruf-coach/wirkung-executive-coaching (10.01.2018)

Deloch, H. \& Feuerstein, H. (2013). Erlebensbezogenes Concept Coaching (ECC) - vielfältige Anwendungen in der Praxis. Die Entwicklung eigenständiger Konzepte und innovativer 
Zusammenarbeit gezielt unterstützen. Gesprächspsychotherapie und Personzentrierte Beratung $1 / 13,35-43$

Deloch, H. (2017). Erlebensbezogen Denken, Coachen und Moderieren: Der Coachingansatz ECC - Erlebensbezogenes Concept-Coaching. Person, 21/2, 120-131

De Meuse, K., Dai, G. \& Lee, R. (2009): Evaluating the effectiveness of executive coaching: Beyond ROI? Coaching: An Inernational Journal of Theory Research an Practice 2(29), 117 134

Dempster, M. \& Hanna, D. (2017): Forschungsmethoden der Psychologie und Sozialwissenschaften für Dummies. Weinheim: Wiley-VCH Verlag

Deplazes, S., Schwyter, S. \& Möller, H. (2016): Ein Blick auf Coachingprozesse - Die Interventionen des Coachs, dargestellt und analysiert mittels des KaSyCo-C. Coaching Theorie \& Praxis. Doi:10.1365/s40896-016-0010-4

Deppermann, A. (2008): Gespräche analysieren. Wiesbaden: VS Verlag für Sozialwissenschaften

Die Bibel (1999): Lutherbibel, revidierter Text 1984, durchgesehene Ausgabe. Stuttgart: Deutsche Bibelgesellschaft

Diener, M.; Hilsenroth, M. \& Weinberger, J. (2007): Therapist Affect Focus and Patient Outcomes in Psychodynamic Psychotherapy: a Meta-Analysis. Am J Psychiatry 164, 936941

Döring, N. \& Bortz, J. (2016). Forschungsmethoden und Evaluation in den Sozial- und Humanwissenschaften. 5. vollständig überarbeitete, aktualisierte und erweiterte Auflage. Berlin: Springer

Dresing, T. \& Pehl, T. (2018): Praxisbuch Interview, Transkription \& Analyse. Anleitungen und Regelsysteme für qualitativ Forschende. 8. Auflage. Marburg. Online unter: https://www.audiotranskription.de/praxisbuch (06.04.2020)

Eberwein, W. (2009). Humanistische Psychotherapie. Quellen, Theorien und Techniken. Stuttgart: Thieme

Ebner, K. (2016). Veränderungen durch Coaching: Wie lernt ein Coachee im Coaching-Prozess? In C. Triebel, J. Heller, B. Hauser \& A. Koch (Hrsg.): Qualität im Coaching. Denkanstöße und neue Ansätze: Wie Coaching mehr Wirkung und Klientenzufriedenheit bringt (S.81-91). Heidelberg: Springer

Elliot, R. (1984). A Discovery-Oriented Approach to Significant Change Events in Psychotherapy: Interpersonal Process Recall and Comprehensive Analysis. In L. Rice, \& L. Greenberg (Hrsg.): Patterns of change: Intensive analysis of psychotherapy process (S. 249-286). New York: Guilford Press

Elliot, R. (2010): Psychotherapy change process research: Realizing the promise. Psychotherapy Research, 20 (2), 123-135

Elliot, R. (2012a). Qualitative Methods für Studying Psychotherapy Change Processes. In A. 
Thompson \& D. Harper (Hrsg.): Qualitative research methods in mental health and psychotherapy: An introduction for students and practitioners (S. 69-81). Chichester: Wiley-Blackwells

Elliot, R. (2012b) Emotion-focused therapy. In: P. Sanders (Hrsg.): The tribes of the personcentred nation. An introduction to the schools of therapy related to the person-centred approach (S. 103-130). Ross-on-Wye: PCCS Books

Elliot, R. \& Freire, E. (2010). The effectiveness of person-centred and experiential therapies. A review of the meta-analyses. In M. Cooper, J. Watson \& D. Hölldampf (Hrsg.): Personcentered and experiential therapies work. A review of the research on counseling, psychotherapy and related practices (S. 1-15). Ross-on-Wye: PCCS Books

Elliot, R. \& Greenberg, L. (2007). The Essence of Process-Experiential / Emotion-Focused Therapy. American Jorunal of Psychotherapy 61/3, 241-254

Elliot, R., Greenberg, L. \& Lietaer, G. (2013). Forschung zu erfahrungsorientierten Psychotherapieansätzen. In M. Lambert (Hrsg.), M. Richard \& H. Vogel (Dt. Hrsg.): Bergin \& Garfields Handbuch der Psychotherapie und Verhaltensmodifikation (S. 791-866). Tübingen: dgvt-Verlag

Elliot, R., Greenberg, L. \& Lietaer, G. (2019). Promising Leads for Resarch. Online unter: http://www.experiential-researchers.org/leads.html (24.08.2019)

Fäh, M. \& Fischer, G. (1998): Sinn und Unsinn in der Psychotherapieforschung: eine kritische Auseinandersetzung mit Aussagen und Forschungsmethoden. Gießen: PsychosozialVerlag

Faltermaier, T., Mayring, P., Saup, W. \& Strehmel, P. (2002). Entwicklungspsychologie des Erwachsenenalters. 2. überarb. und erw. Auflage. Stuttgart: Kohlhammer

Feuerstein. H. \& Müller, D. (1998). Supervision als Prozess. Beiträge des Focusing-Ansatzes zu Theorie und Praxis Personzentrierter Supervision. In U. Straumann \& W. Schröder (Hrsg.): Verstehen und Gestalten: Beratung und Supervision im Gespräch (S. 37-52). Köln: GwG-Verlag

Feuerstein, H. \& Müller, D. (2000). Focusing und erlebensbezogene Methode - eine Einführung. Gesprächspsychotherapie und Personzentrierte Beratung, 2/00, 96-103

Feuerstein, H. \& Deloch, H. (2010). Erlebensbezogenes Denken: Neue Konzepte entwickeln in Beratungsprozessen. Gesprächspsychotherapie und Personzentrierte Beratung 4/2010, 1-7 (Sonderdruck)

Fietze, B. (2015): Coaching auf dem Weg zur Profession? Eine professionssoziologische Einordnung. In A. Schreyögg \& C. Schmidt-Lellek (Hrsg.): Die Professionalisierung von Coaching. Ein Lesebuch für den Coach (S. 3-21). Wiesbaden: Springer

Fischer-Epe, M. (2002). Coaching: Miteinander Ziele erreichen. Reinbek bei Hamburg, Rowohlt Taschenbuch Verlag

Fonagy, R., Gergely, G., Jurist, E. \& Target, M. (2002). Affect Regulation, Mentalization 
and the Development of the Self. New York: Other Press

Friesenhahn, J. (2017). Kommunikation als Basis wirkungsvollen FührungskräfteCoachings. Von der Dyade zur Triade im Setting mit Pferden. Wiesbaden, Springer

Fuchs, M. (1974). Funktionelle Entspannung: Theorie und Praxis einer organismischen Entspannung über den rhythmisierten Atem. Stuttgart: Hippokrates

Gendlin, E. (1968): The Experiential Response. In: E. Hammer (Hrsg.): Use of Interpretation in Treatment. Technique and Art (S. 208-227). New York: Grune \& Stratton

Gendlin, E. (1974). Client-centered and experiential psychotherapy. In. D. Wexeler \& L. Rice (Hrsg.): Innovations in client-centered therapy (S. 211-246). New York: Wiley

Gendlin, E. (1978). Eine Theorie der Persönlichkeitsveränderung. In H. Bommert \& H. Dahlhoff (Hrsg.): Das Selbsterleben (Experiencing) in der Psychotherapie (S. 1-62). München: Urban und Schwarzenberg

Gendlin, E. (1986). What comes after traditional psychotherapy research? American Psychologist 41 (2), 131-136. Online unter: https://www.focusing.org/gendlin/docs/gol_2058.html (20.08.2019)

Gendlin, E. (1996). Focusing-orientierte Psychotherapie. Ein Handbuch der erlebensbezogenen Methode. München: Pfeiffer

Gendlin, E. (1998). Focusing. Selbsthilfe bei der Lösung persönlicher Probleme. Hamburg: Rowohlt

Gendlin, E. (2002). The Experiential Response. Teil I: Regeln für das therapeutische Antworten. Focusing-Journal 8, 1-11

Gendlin, E. (2004). Einführung in Thinking at the Edge. The Folio: A Journal for Focusing and Experiential Therapy. 19(1), 1-8. Online unter: http://previous.focusing.org/de/TAE_Einfuhrung_Folio_de_HPM10.pdf (11.05.2020)

Gendlin, E. (2015): Ein Prozess-Modell: Körper. Sprache. Erleben. Freiburg: Karl Alber

Gendlin, E., \& Hendricks, M. (2004). Thinking at the Edge (TAE) Steps. The Folio: A Journal for Focusing and Experiential Therapy. 19(1), 12-24

Gendlin, E. \& Wiltschko, J. (2016). Focusing in der Praxis. Eine schulenübergreifende Methode für Psychotherapie und Alltag. 6. Auflage. Stuttgart: Klett-Cotta

Gessnitzer, S. \& Kauffeld, S. (2015). The Working Alliance in Coaching: Why Behavior Is the Key to Success. The Journal of Applied Behavioral Science, 51(2), 177-197

Gessnitzer, S \& Kauffeld, S. (2018). Coaching. Wissenschaftliche Grundlagen und praktische Anwendung. Stuttgart: Kohlhammer

Gessnitzer, S., Schulte, E. \& Kauffeld, S. (2018). Psychologische Coaching-Forschung zu Interaktionsprozessen, Coaching-Formaten und Diagnoseinstrumenten. Empirische Erkenntnisse als Anregungen für die Coaching-Praxis der Zukunft. In R. Wegener, A. Fritze, M. Hänseler \& M. Loebbert (Hrsg.): Coaching-Prozessforschung. Forschung und Praxis im Dialog. (S. 37-55). Göttingen: Vandenhoeck \& Ruprecht 
Geuter, U. (2015). Körperpsychotherapie. Grundriss einer Theorie für die klinische Praxis. Berlin: Springer

Gläser, J. \& Laudel, G. (1999): Theoriegeleitete Textanalyse? Das Potential einer variablenorientierten qualitativen Inhaltsanalyse. WZB Discussion Paper, No. P 99-401. Online unter: http://hdl.handle.net/10419/50917 (31.01.2019)

Goldmann, B. (2014). Selbstanteile - eine Herausforderung in Theorie und Praxis der Personzentrierten Psychotherapie. Person 18(2), 127-138

Gollino, L. (2016). A task analysis of how clients formulate reappraisals in cognitive-behavior therapy for depression. Thesis for the degree of Doctor of Philosophy, Ontario Institute for Studies in Education, University of Toronto

Gonçalves, M., Matos, M. \& Santos, A. (2009). Narrative therapy and the nature of ,innovative moments" in the construction of change. Journal of Constructivist Psychology, 22, 123

Gonçalves, M., Mendes, I., Ribeiro, A., Angus, L. \& Greenberg, L. (2010). Innovative moments and change in emotional focused therapy: The case of Lisa. Journal of Constructivist Psychology, 23, 1-28

Graf, E. (2015): Kommunikative Basistätigkeiten im Coaching-Gespräch: Ein linguistischer Beitrag zur Coaching-Prozessforschung. Coaching Theorie \& Praxis I:5-14. DOI $10.1365 / \mathrm{s} 40896-015-0001-\mathrm{x}$

Graf, E. (2018): Äußerung für Äußerung zum Coaching-Prozess. Das Potenzial einer gesprächsanalytisch fundierten Coaching-Prozessforschung. In R. Wegener, A. Fritze, M. Hänseler \& M. Loebbert (Hrsg.): Coaching-Prozessforschung. Forschung und Praxis im Dialog (S.137-135). Göttingen: Vandenhoeck \& Ruprecht

Grant, A. (2014): ROI is a Poor Measure of Coaching Success: Towards a More Holistic Approach Using a Well-being and Engagement Framework. In: B. Garvea (Hrsg.): Fundamentals of Coaching and Mentoring. Researching Coaching and Mentoring. Volume III. Los Angeles: SAGE Library in Business \& Management

Graßmann, C., Schölmerich, F. \& Schermuly, C. (2019). The relationship between working alliance and client out-comes in coaching: A meta-analysis. Human Relations Advance Online Publication. DOI 10.1177/0018726718819725. Online unter https://journals.sagepub.com/doi/10.1177/0018726718819725 (19.12.2019)

Greenberg, L. (1984a). A Task Analysis of Intrapersonal Conflict Resolution. In L. Rice, \& L. Greenberg (Hrsg.): Patterns of change: Intensive analysis of psychotherapy process (S. 67-123). New York: Guilford Press

Greenberg, L. (1984b). Task Analysis: The General Approach. In L. Rice, \& L. Greenberg (Hrsg.): Patterns of change: Intensive analysis of psychotherapy process (S. 67-123). New York: Guilford Press 
Greenberg, L. (1986): Change process research. Journal of Consulting \& Clinical Psychology, 54, 4-9

Geenberg. L. (2000). Von der Kognition zur Emotion in der Psychotherapie. In: S. Sulz \& G. Lenz (Hrsg.): Von der Kognition zur Emotion. Psychotherapie mit Gefühlen (S. 77-110). München: CIP-Medien

Greenberg, L. (2006). Emotionsfokussierte Therapie: Lernen, mit den eigenen Gefühlen umzugehen. Tübingen: dgvt-Verlag

Greenberg, L. (2007). A guide to conducting a task analysis of psychotherapeutic change. Psychotherapy Research, 17(1), 15-30

Greenberg, L., Rice, L. \& Elliot, R. (2003). Emotionale Veränderung fördern. Grund-lagen einer prozess- und erlebensorientierten Therapie. Paderborn: Junfermann

Greif, S. (2008). Coaching und ergebnisorientierte Selbstreflexion. Theorie, Forschung und Praxis des Einzel- und Gruppencoachings. Göttingen: Hogrefe

Greif, S., Möller, H. \& Scholl, W. (2018). Coachingdefinitionen und -konzepte. In: S. Greif, H. Möller \& W. Scholl (Hrsg.): Handbuch Schlüsselkonzepte im Coaching (S. 1-9). Berlin: Springer

Greif, S. \& Rauen, C. (2018): Selbstreflexion im Coaching. In: S. Greif, H. Möller \& W. Scholl (Hrsg.): Handbuch Schlüsselkonzepte im Coaching (S. 523-531). Berlin: Springer

Greif, S. \& Riemenschneider-Greif, F. (2018). Wie im Coaching neue Einsichten entstehen. Anregung für eine theoriegeleitete Erforschung innerpsychischer Prozesse. In R. Wegener, A. Fritze, M. Hänseler \& M. Loebbert (Hrsg.): Coaching-Prozessforschung. Forschung und Praxis im Dialog. (S. 112-136). Göttingen: Vandenhoeck \& Ruprecht

Greisel, M. (2015): Prozesstheorie am Einzelfall prüfen? Ein hermeneutisches Verfahren auf der Basis quantitativer Daten. Journal für Psychologie, 23, 2

Gugutzer, R. (2012): Verkörperung des Sozialen. Neophänomenologische Grundlagen und soziale Analysen. Bielefeld: transcript

Hauke, G. (2011): Kognitiv-affektive Schemata im Coaching. In: B. Birgmeier (Hrsg.): Coachingwissen. 2., aktualisierte und erweiterte Auflage (S. 227-242). Wiesbaden: VS Verlag für Sozialwissenschaften

Heidenreich, T. \& Michalak, J. (2013). Die »dritte Welle« der Verhaltenstherapie: Grundlagen und Praxis. Weinheim: Beltz

Heidenreich, T., Mundle, G. \& Michalak, J. (2013). Achtsamkeitsbasierte Rückfallprävention im Suchtbereich. In: T. Heidenreich \& J. Michalak (Hrsg.): Die „dritte Welle“ der Verhaltenstherapie. Grundlagen und Praxis (S. 150-164). Weinheim: Beltz

Hein, M. \& Sewz, G. (2005). Wissenschaftstheorie und Ethik (Studienbrief 77354). Hagen: Fernuniversität.

Hendricks, M. (2002). Focusing-oriented/experiential psychotherapy. In D. Cain (Hrsg.): Humanistic psychotherapies: Handbook of research and practice (S. 221-251). Washington: 
American Psychological Association. Online unter http://previous.focusing.org/research_basis.html (Zugriff 09.01.2020)

Hinz, A. \& Behr, M. (2002). Biografische Rekonstruktionen und Reflexionen. Zum 100. Geburtstag von Carl Rogers. Gesprächspsychotherapie und Personzentrierte Beratung, 3/2002, 197-210

Höher, F. \& Steenbuck, G. (2006). Personenbezogene Beratung und regionale Strukturpolitik - Ein integratives Beratungskonzept zur Work-Life-Balance. Gesprächspsychotherapie und Personzentrierte Beratung 2/06, 94-99

Hofmann, T. (2016). Experienzielle Kommunikation. Wie kann soziales Miteinander in komplexen Situationen gelingen? Coburg: ZKS-Verlag

Hüther, G. \& Dohne, K. (2011). Wer sich weiterentwickeln will, kann nicht so weitermachen wie bisher. In: W. Leeb, B. Trenke \& M. Weckenmann (Hrsg.): Der Realitätenkellner. Hypnosystemische Konzepte in Beratung, Coaching und Supervision (S. 36-52). Heidelberg: Carl Auer

Hummitzsch, H. (2001). Personzentrierte Organisationsentwicklung aus systemtheoretischer (Synergetischer) Perspektive unter besonderer Berücksichtigung des von Beatrix Terjung entwickelten Verfahrens. In B. Terjung \& T. Kempf (Hrsg.): Von der Klientenzentrierten Therapie zur Personzentrierten Organisationsentwicklung (S. 3 - 17). Köln: GWG-Verlag

Iberg, J. (1996). Using statistical experiments with post-session client questionnaires as a student-centred approach to teaching the effects of therapist activities in psychotherapy. In $\mathbf{R}$. Hutterer, P. Pawlowski, P. Schmid \& R. Stipsits (Hrsg.): Client-centered and experiential psychotherapy. A paradigm in motion (S. 255-271). Frankfurt: Peter Lang

Jaison, B. \& Lawlor, M. (1996). Focusing and research. The Folio: A Journal for Focusing and Experiential Therapy, 15(1), 1-84

Jones, G. \& Bouncken, R. (2008). Organisation. Theorie, Design und Wandel. 5. aktualisierte Auflage. München: Pearson

Jones, R., Woods, S. \& Guillaume, Y. (2015): The effectiveness of workplace coaching: A meta-analysis of learning and performance outcomes from coaching. Journal of Occupational and Organizational Psychology 89, 249-277. DOI:10.1111/joop.12119

Kabat-Zinn, J. (1990). Full catastrophe living: Using the wisdom of your body and mind to face stress, pain and illness. New York: Delacorte

Kauffeld, S. \& Gessnitzer, S. (2018): Coaching. Wissenschaftliche Grundlagen und praktische Anwendung. Stuttgart: Kohlhammer

Kern, E. (2015): Personzentrierte Körperpsychotherapie. Gesprächspsychotherapie und Personzentrierte Beratung 15(3), 134-140

Kilburg, R. (2001). Facilitating intervention adherence in executive coaching: A model and methods. Consulting Psychology Journal: Practice and Research, 53(4), 251-267

Kirkpatrick, D. (1967). Evaluation of training. In: R. Craig \& L. Bittel (Hrsg.): Training 
and Development Handbook (S. 87-112). New York: McGraw Hill

Klein, M., Mathieu, P., Gendlin, E. \& Kiesler, D. (1969): The Experiencing Scale: A research and training manual. Madison: University of Wisconsin, Extension Bureau of Audiovisual instruction

Koch, P. (2006). Qualitative Methoden in der Arbeits- und Organisationspsychologie. (Studienbrief 77368-8-01-S1). Hagen: Fernuniversität

Koch, S. (2011). Embodiment. Der Einfluss von Eigenbewegung auf Affekt, Einstellung und Kognition. Empirische Grundlagen und klinische Anwendungen. Berlin: Logos Verlag

Kotte, S., Hinn, D., Oellerich, K. \& Möller, H. (2016): Der Stand der Coachingforschung: Kernergebnisse der vorliegenden Metaanalysen. Organisationsberatung Supervision Coaching 1:5-23. DOI 10.1007/s11613-016-0444-6

Kotte, S., Hinn, D., Oellerich, K. \& Möller, H. (2018): Stand der Coachingforschung: Ergebnisse der vorliegenden Metaanalysen. In: S. Greif, H. Möller \& W. Scholl (Hrsg.): Handbuch Schlüsselkonzepte im Coaching (S. 553-562). Berlin: Springer

Kotte, S., Müller, A. \& Hennemann, L. (2019). Die Kasseler Coachingstudie. Informationsschreiben zur Kasseler Coachingstudie, Online unter: https://www.uni-kassel.de/fb01/fileadmin/datas/fb01/Institut_fuer_Psychologie/Dateien/Methoden/Kasseler_Coaching-Studie_Projektflyer_2019_08.pdf (21.11.2019)

Kotte, S., Oellerich, K., Schubert, D. \& Möller, H. (2015): Das ambivalente Verhältnis von Coachingforschung und -praxis: Dezentes Ignorieren, kritisches Beäugen oder kooperatives Miteinander? In A. Schreyögg \& C. Schmidt-Lellek (Hrsg.): Die Professionalisierung von Coaching. Ein Lesebuch für den Coach (S. 23-45). Wiesbaden: Springer.

Kottler, J. (2014). Change: What really leads to lasting personal transformation. New York: Oxford University Press

Kriz, J. (2010): Personzentrierte Systemtheorie. Person. Internationale Zeitschrift für Personzentrierte und Experienzielle Psychotherapie und Beratung. 14(2), 99-113

Kuckartz, U., Dresing, T., Rädiker, S. \& Stefer, C. (2007). Qualitative Evaluation. Der Einstieg in die Praxis. Wiesbaden: VS Verlag für Sozialwissenschaften

Künzli, H. (2013): Wirksamkeitsforschung im Führungskräftecoaching. In: Lippmann: Coaching. Angewandte Psychologie für die Beratungspraxis. 3. überarbeitete Auflage. Berlin, Heidelberg: Springer-Verlag

Künzli, H. \& Stulz, N. (2011): Individuumsorientierte Coachingforschung. In: B. Birgmeier (Hrsg.): Coachingwissen. 2., aktualisierte und erweiterte Auflage (S. 161-172). Wiesbaden: VS Verlag für Sozialwissenschaften

Kunze, D. (2016). Personzentriertes Coaching: Veränderung durch Beziehung. Die KlientCoach-Beziehung im Mittelpunkt. Coaching-Magazin 1/2016, 20-25

Kurtz, R. (1985). Körperzentrierte Psychotherapie: die Hakomi Methode. Essen: SynthesisVerlag 
Lamnek, S. (2005). Qualitative Sozialforschung. Lehrbuch. 4. überarbeitete Auflage. Weinheim: Beltz

Landis, J. \& Koch, G. (1977). The Measurement of Observer Agreement for Categorical Data. Biometrics, 33(1), 159. Doi:10.2307/2529310

Langer, I. (2000). Das Persönliche Gespräch als Weg in der psychologischen Forschung. Köln: GWG-Verlag

Lazarus, R. \& Launier, R. (1981). Stressbezogene Transaktionen zwischen Person und Umwelt. In J. Nitsch (Hrsg.): Stress: Theorien, Untersuchungen, Maßnahmen (S. 123-259). Bern: Huber

Le Coutre, C. (2016). Focusing zum Ausprobieren. Eine Einführung für psychosoziale Berufe. München: Ernst Reinhardt Verlag

Leuzinger-Bohleber, M., Benecke, C. \& Hau, S. (2015): Psychoanalytische Forschung. Methoden und Kontroversen in Zeiten wissenschaftlicher Pluralität. Stuttgart: Kohlhammer Lex, S. \& Bischkopf., J. (2012). Wie Gefühlsprozesse in der Therapie methodisch erfasst werden können. Gesprächspsychotherapie und Personzentrierte Beratung 4/12, 207-213

Limmer, A. \& Schütz, A. (2018): Resilienz - Modewelle oder Paradigmenwechsel in Prävention und Coaching? In: S. Greif, H. Möller \& W. Scholl (Hrsg.): Handbuch Schlüsselkonzepte im Coaching (S. 485-493). Berlin: Springer.

Lincoln, Y. \& Guba, E. (1985). Naturalistic Inquiry. Newbury Park: Sage

Lindart, M. (2016): Was Coaching wirksam macht. Wirkfaktoren von Coachingprozessen im Fokus. Wiesbaden: Springer

Loebbert, M., Wegener, R., Fritze, A. \& Hänseler, M. (2018). Einleitung der Herausgeberinnen und Herausgeber. In R. Wegener, A. Fritze, M. Hänseler \& M. Loebbert (Hrsg.): Coaching-Prozessforschung. Forschung und Praxis im Dialog. (S. 11-18). Göttingen: Vandenhoeck \& Ruprecht

Loos, Wolfgang (2006). Unter vier Augen: Coaching für Manager. Bergisch Gladbach: EHP - Verlag Andreas Kohlhage

Lowen, A. (2008). Bioenergetik: Therapie der Seele durch Arbeit mit dem Körper. Reinbek: Rowohlt

Lowry, R. (2020). VassarStats: Website for Statistical Computation. Online unter: http://vassarstats.net/ (25.05.2020)

Mäthner, E., Jansen, A \& Bachmann, T. (2005). Wirksamkeit und Wirkung von Coaching. In C. Rauen (Hrsg.), Handbuch Coaching, 3. Auflage (S. 55-76). Göttingen: Hogrefe

Mathieu-Coughlan, P. \& Klein, M. (1984). Experiential Psychotherapy: Key Events in Client-Therapist Interaction. In L. Rice, \& L. Greenberg (Hrsg.): Patterns of change: Intensive analysis of psychotherapy process (S. 213-248). New York: Guilford Press

Maurer, I. (2006). Verhaltenssteuerung durch emotionale Selbstregulation im Coaching. In: 
U. Straumann \& C. Zimmermann-Lotz (Hrsg.): Personzentriertes Coaching und Supervision - ein interdisziplinärer Balanceakt. Kröning: Asanger

Maurer, I. (2013). Personzentriertes Coaching. In: S. Gahleitner, I. Maurer, E. Ploil \& U. Straumann (Hrsg.): Personzentriert beraten: alles Rogers? Theoretische und praktische Weiterentwicklungen (S. 144-159). Weinheim: Beltz Juventa

Mayring, P. (2001). Kombination und Integration qualitativer und quantitativer Analyse. FQS Forum qualitative Sozialforschung 2(1), Art. 6. DOI: http://dx.doi.org/10.17169/fqs2.1.967

Mayring, P. (2007): Qualitative Inhaltsanalyse. Grundlagen und Techniken. Weinheim und Basel: Beltz Verlag

Mearns, D. (1999). Person-centred therapy with configurations of self. Counselling, 10(2), $125-130$

Meibert, P., Michalak, J. \& Heidenreich, T. (2013). Stressbewältigung durch Achtsamkeit: MBSR. In: T. Heidenreich \& J. Michalak (Hrsg.): Die „dritte Welle“ der Verhaltenstherapie. Grundlagen und Praxis (S. 165-179). Weinheim: Beltz

Meibert, P. (2016). Achtsamkeitsbasierte Therapie und Stressreduktion MBCT/MBSR. München: Ernst Reinhardt Verlag

Mendes, I., Ribeiro, A., Agnus, L., Greenberg, L., Sousa, I. \& Gonçalves, M. (2011). Narrative change in emotion-focused psychotherapy: A study on the evolution of reflection and protest innovative Moments. Psychotherapy Research 21/3, 304-315, DOI: $10.1080 / 10503307.2011 .565489$

Michalak, J. \& Heidenreich, T. (2013). Achtsamkeitsbasierte Kognitive Therapie (MBCT). In: T. Heidenreich \& J. Michalak (Hrsg.): Die „dritte Welle“ der Verhaltenstherapie. Grundlagen und Praxis (S. 121-138). Weinheim: Beltz

Migge, B. (2007). Handbuch Coaching und Beratung. 2. Auflage. Weinheim und Basel: Betz Verlag

Misoch, S. (2015): Qualitative Interviews. Berlin: De Gruyter Oldenbourg

Möller, H. \& Kotte, S. (2011): Die Zukunft der Coachingforschung. OSC Organisationsberatung Supervision Coaching, 18, 445-456

Orlinski, D., Ronnestad, M. \& Willutzki, R. (2013). 50 Jahre Prozess-Outcome-Forschung: Kontinuität und Wandel. In M. Lambert (Hrsg.), M. Richard \& H. Vogel (Dt. Hrsg.): Bergin \& Garfields Handbuch der Psychotherapie und Verhaltensmodifikation (S. 501-626). Tübingen: dgvt-Verlag

Pascual-Leone, A. \& Yeryomenko, N. (2017). The client "experiencing" scale as a predictor of treatment outcomes: A meta-analysis on psychotherapy process, Psychotherapy Research 27(6), 653-665, DOI: 10.1080/10503307.2016.1152409

Pascual-Leone, A., Greenberg, L. \& Pascual-Leone, J. (2009). Developments in task analysis: New methods to study change. Psychotherapy Research 19(4-5), 527-542 
Passmore, J. (2007). An integrative model for executive coaching. Consulting Psychology Journal: Practice and Research, 59(1), 68-78

Perler, D. (1996): War Aristoteles ein Funktionalist? Überlegungen zum Leib-Seele-Phänomen. Zeitschrift für philosohische Forschung 50, 341-363

Perls, F. (2018). Gestalt-Therapie in Aktion. 12. Auflage. Stuttgart: Klett-Cotta.

Petzold, H. (2006). Der ,informierte Leib“: „embodied and embedded“ - ein Metakonzept für die Leibtherapie. In G. Marlock \& H. Weiss (Hrsg.): Handbuch der Körperpsychotherapie (S. 100-118). Stuttgart: Schattauer

Piaget, J. (1976). Die Äquilibration der kognitiven Strukturen. Stuttgart: Klett

Purton, C. (2004). Person-centred therapy. The focusing-oriented approach. New York: Palgrave Macmillan

Renn, K. (2016). Magische Momente der Veränderung. Was Focusing bewirken kann. Eine Einführung. München: Kösel-Verlag

Reinecker, H. (1984). Prozesstheorien: Verhaltensorientierte Modelle. In: U. Baumann (Hrsg.): Psychotherapie: Makro-/Mikroperspektive (S. 159-176). Göttingen: Verlag für Psychologie

Rice, L., \& Greenberg, L. (1984). The New Research Paradigm. In: L. Rice \& L. Greenberg (Hrsg.): Patterns of change: Intensive analysis of psychotherapy process (S. 7-25). New York: Guilford Press.

Rice, L. \& Saperia, E. (1984). Task Analysis of the Resolution of Problematic Reactions. In: L. Rice \& L. Greenberg (Hrsg.): Patterns of change: Intensive analysis of psychotherapy process (S. 29-66). New York: Guilford Press.

Riva, G. (2015). Phenomology of Positive Change: Personal Growth. In: P. Inghilleri; G. Riva \& E. Riva (Hrsg.): Enabling Positive Change. Flow and Complexity in Daily Experience. Warschau, Berlin: De Gruyter Open

Rogers, C. (1972). Die nicht-direktive Beratung. München: Kindler

Rogers, C. (1991b). Eine Theorie der Psychotherapie, der Persönlichkeit und der zwischenmenschlichen Beziehungen (3. Auflage 1991, Nachdruck 2002). Köln: GWG-Verlag

Rogers, C. (2000). Therapeut und Klient (15. Auflage). Frankfurt: Fischer

Rogers, C. (2008). Eine Theorie der Psychotherapie, der Persönlichkeit und der zwischenmenschlichen Beziehungen. München: Reinhardt/GwG

Roth, G. \& Ryba, A. (2016). Coaching, Beratung und Gehirn. Neurobiologische Grundlagen wirksamer Veränderungskonzepte. Stuttgart: Klett-Kotta

Roth, G. \& Ryba, A. (2017). Coaching und Neurowissenschaften. Coaching-Newsletter Juni 2017. Online unter: https://www.coaching-newsletter.de/archiv/2017/coaching-newsletterjuni-2017.html\#c14650 (07.11.2019)

Roth, G. (2019). Integratives Coaching auf neurowissenschaftlicher Grundlage. OSC Organisationsberatung Supervision Coaching 26, 297-312 
Ryba, A. (2018). Die Rolle unbewusster und vorbewusst-intuitiver Prozesse im Coaching. Göttingen: Vandenhoeck \& Ruprecht

Ryba, A. (2019). Körperzentriertes Coaching und der Zugang zum Unbewussten. OSC Organisationsberatung Supervision Coaching 26, 313-329

Sachse, R., Atrops, A., Wilke, F. \& Maus, C. (1992): Focusing: ein emotionsorien-tiertes Psychotherapie-Verfahren. Bern: Verlag Hans Huber

Sachse, R. \& Fasbender, J. (2011). Focusing: Eine Therapietechnik zur Repräsentation affektiver Schemata. In: R. Sachse, J. Fasbender, J. Breil \& M. Sachse (Hrsg.): Perspektiven Klärungsorientierter Psychotherapie II (S. 131-155). Lengerich: Pabst

Sachse, R. \& Langens, T. (2014). Emotionen und Affekte in der Psychotherapie. Göttingen: Hogrefe

Safran, J., Muran, C. \& Samstag, L. (1994). Resolving therapeutic alliance ruptures: A task analytic investigation. In: A. Horvath \& L. Greenberg (Hrsg.): The working alliance: Theory, research and practice (S. 225-255). New York: Wiley

Sander, K. \& Ziebertz, T. (2010). Personzentrierte Beratung. Ein Lehrbuch für Ausbildung und Praxis. München: Juventa

Schindler, L. (1991): Die empirische Analyse der therapeutischen Beziehung. Beiträge zur Prozessforschung in der Verhaltenstherapie. Berlin Heidelberg: Springer-Verlag

Schmidt, G. (2011). Berater als „Realitätskellner“ und Beratung als koevolutionäres Konstruktionsritual für zieldienliche Netzwerkaktivierungen - Einige hynosystemische Implikationen. In: W. Leeb, B. Trenke \& M. Weckenmann (Hrsg.): Der Realitätenkellner. Hypnosystemische Konzepte in Beratung, Coaching und Supervision (S. 18-35). Heidelberg: Carl Auer

Schmidt, G. (2014). Einführung in die hypnosystemische Therapie und Beratung. Sechste Auflage. Heidelberg: Carl Auer

Scholl, W. \& Kunert, S. (2018). Interaktion als Grundlagenthema im Coaching. In: S. Greif, H. Möller \& W. Scholl (Hrsg.): Handbuch Schlüsselkonzepte im Coaching (S. 265-273). Berlin: Springer

Schütze, F., Fiedler, W. (Hrsg.) \& Krüger, H. (Hrsg.) (2016): Sozialwissenschaftliche Prozessanalyse: Grundlagen der qualitativen Sozialforschung. Leverkusen: Verlag Barbara Budrich

Schulz von Thun, F. (2014). Miteinander reden - 3: Das „Innere Team“ und situationsgerechte Kommunikation: Kommunikation, Person, Situation. Reinbek: Rowohlt

Schwarz, R. (2015): Applied Embodiement und das Konzept der Leiblichkeit in Beratung, Supervision und Coaching. Resonanzen. E-Journal für biopsychosoziale Dialoge in Psychotherapie, Supervision und Beratung. 3(1), 52-64. Online unter: http://www.resonanzen-journal.org (29.12.2019) 
Schwarz, R. (2017): Embodiment und leibliche Kommunikation in der Beratung. Supervision, 35(1), 66-71

Seewald, C. (2006). Personzentriertes Coaching fördert das "latent Gewusste" ans Tageslicht und löst Blockaden. Gesprächspsychotherapie und Personzentrierte Beratung 2/06, 69-70

Sonesh, S., Coultas, C., Lacerenza, C., Marlow, S., Benishek, L. \& Salas, E. (2015). The power of coaching: A meta-analytic investigation. Coaching: An International Journey of Theory, Research and Practice, 8, 73-95

Stanovich, K. \& West, R. (2000). Individual differences in reasoning: implications for the rationality debate? Behavioral and Brain Sciences, 23(5), 645-726

Starker, U. \& Müller, J. (2018). Komplexes Problemlösen im Coaching. In: S. Greif, H. Möller \& W. Scholl (Hrsg.): Handbuch Schlüsselkonzepte im Coaching (S. 307-314). Berlin: Springer

Steinhage, R. (2005): Personzentrierte Kommunikation im Coaching. Gesprächspsychotherapie und Personzentrierte Beratung, 3/2005, 175-177.

Steinke, I. (2007). Gütekriterien qualitativer Forschung. In: U. Flick, E. v. Kardorff \& I. Steinke (Hrsg.): Qualitative Forschung. Ein Handbuch (S. 319-331). Reinbek bei Hamburg: Rowohlt

Stiles, W. (2007). Theory-building case studies of counselling and psychotherapy. Counselling and Psychotherapy Research, 7(2), 122-127. DOI: 10.1080/14733140701356742

Stolze, H. \& Badura-MacLean, E. (1984). Die konzentrative Bewegungstherapie: KBT; Grundlagen und Erfahrungen. Berlin: Verlag Mensch u. Leben

Storch, M., Cantieni, B., Hüther, G. \& Tschacher, W. (2006). Embodiment. Die Wechselwirkung von Körper und Psyche verstehen und nutzen. Bern: Hans Huber

Strauss, A. \& Corbin, J. (1990). Basics of qualitative research: Grounded theory procedures and techniques. Thousand Oaks: Sage Publications

Taubner, S. \& Kotte, S. (2018): Mentalisierung im Coaching. In: S. Greif, H. Möller \& W. Scholl (Hrsg.): Handbuch Schlüsselkonzepte im Coaching (S. 353-362). Berlin: Springer

Tausch, R. \& Tausch, A. (1973). Erziehungspsychologie: psychologische Prozesse in Erziehung und Unterrichtung. Göttingen: Hogrefe

Theeboom, T., Beersma, B. \& van Vianen, A. (2014). Does coaching work? A meta-analysis on the effects of coaching on individual level outcomes in an organizational context. The Journal of Positive Psychology, 9, 1-18

The International Focusing Institute (2019). Focusing \& Coaching: Exploring the Possibilities of Focusing-Oriented Coaching. Part of the Focusing Roundtable Series. Online unter: https://focusing.org/civicrm-event/51 (14.08.2019)

Thoma, H. \& Kächele, H. (2006). Psychoanalytische Therapie. Forschung. Heidelberg. Springer Medizin Verlag 
Timulak, L. \& Creaner, M. (2010). Qualitative Meta-Analysis of outcomes of person.centred and experiential psychotherapies. In M. Cooper, J. Watson \& D. Hölldampf (Hrsg.): Person-centered and experiential therapies work. A review of the research on counseling, psychotherapy and related practices (S. 65-90). Ross-on-Wye: PCCS Books

Tolman, E. (1948). Cognitive maps in animals and man. Psychological Review, 55, 189-208 Truax, C.\& Carkhuff, R. (1967). Toward effective counseling and psychotherapy. Chicago: Aldine

Tschacher, W. (2006). Wie Embodiment zum Thema wurde. In: M. Storch, B. Cantieni, G. Hüther \& W. Tschacher (2006): Embodiment. Die Wechselwirkung von Körper und Psyche verstehen und nutzen (S. 11-34). Bern: Hans Huber

Waldrich, S. \& Schley, K. (2019). Experienzielle Verhaltenstherapie mit Kindern und Jugendlichen. Praktische Erfahrungen und theoretische Erläuterungen. PsychotherapeutenJournal 3/2019, 244-252

Wahl, A. (2018). Selbstorganisation fördern in der beruflichen Beratung. Die Synergetik als metatheoretisches Rahmenkonzept zur Fundierung eines integrativen Beratungsansatzes. Dissertationsschrift im Fach Erziehungswissenschaft an der Fakultät für Verhaltens- und Empirische Kulturwissenschaften der Ruprecht-Karls-Universität Heidelberg.

Watson, J., Greenberg, L. \& Lietaer, G. (2010). Relating Process to outcome in personcentred and experiential psychotherapies. The role of the relationship conditions and clients' experiencing. In M. Cooper, J. Watson \& D. Hölldampf (Hrsg.): Person-centered and experiential therapies work. A review of the research on counseling, psychotherapy and related practices (S. 132-163). Ross-on-Wye: PCCS Books

Wegener, R. (2018). Konturen einer prozessorientierten Coaching-Forschung. In R. Wegener, A. Fritze, M. Hänseler \& M. Loebbert (Hrsg.): Coaching-Prozessforschung. Forschung und Praxis im Dialog. (S. 19-36). Göttingen: Vandenhoeck \& Ruprecht

Wegener, R. (2019). Bedeutsame Momente im Coaching. Eine explorative Untersuchung zur Weiterentwicklung der Prozessforschung. Wiesbaden: Springer Open Access. DOI https://doi.org/10.1007/978-3-658-25687-6

Weinberger, S. (1994). Klientenzentrierte Gesprächsführung: eine Lern- und Praxisanleitung für helfende Berufe (6., überarbeitete und erweiterte Auflage). Weinheim: Beltz

Weiss, A. (2011). Psychoanalytische Einzelfall-Psychotherapieprozessforschung. Die Veränderung der Bindungsqualität und der Fähigkeit der Mentalisierung in Psychoanalysen. Dissertationsschrift an der Ludwig-Maximilians-Universität München

Weiss, H. (2013). Zum Studium des Unbewussten über den Körper. Wie die sorgfältige Anwendung der Achtsamkeit einen verfeinerten Arbeitsmodus für die Körperpsychotherapie bereitstellt. In: M. Thielen (Hrsg.): Körper - Gruppe - Gesellschaft. Neue Entwicklungen in der Körperpsychotherapie (S. 257-275). Gießen: Psychosozial-Verlag

Weiss, H., Johanson, G. \& Monda, L. (Hrsg.) (2019). Hakomi - Achtsamkeitszentrierte 
Körpertherapie. Theorie und Praxis. Stuttgart: Klett-Kotta

West-Leuer, B. (2015): Emotionen im Kontext von Coaching. In A. Schreyögg \& C. Schmidt-Lellek (Hrsg.): Die Professionalisierung von Coaching (S. 325-339). Ein Lesebuch für den Coach. Wiesbaden: Springer

Wild-Missong, A. (1996). Focusing Bibliothek. Mit Focusing im Leben. Focusing und Selbstentfaltung, Feminismus, Spiritualität, Schamanismus u.a. Würzburg: Dt. Ausbildungsinst. für Focusing-Therapie

Will, T., Gessnitzer, S. \& Kauffeld, S. (2016). You think you are an empathic coach? Maybe you should think again. The difference between perceptions of empathy vs. empathic behaviour after a person-centred coaching training, Coaching: An International Journal of Theory, Research and Practice, 9(1), 53-68, DOI: 10.1080/17521882.2016.1143023

Wilmes, C. \& Loebbert, M. (2013). Coaching als Beratung. In: M. Loebbert (Hrsg.): Professional Coaching. Konzepte, Instrumente, Anwendungsfelder (S. 17-48). Stuttgart: Schäffer-Poeschel

Wiltschko, J. (2011). Experiencing-Theorie und Focusing-Therapie. Konzepte und Methoden im Umgang mit strukturgebundenem Erleben. In J. Kriz \& T. Slunecko (Hrsg.): Gesprächspsychotherapie, die therapeutische Vielfalt des personzentrierten Ansatzes (S. 95108). Wien: Facultas

Wiltschko, J. (2017). Ist „,der Prozess“ zielorientiert? Essentials aus der focusingtherapeutischen Praxis. Gesprächspsychotherapie und Personzentrierte Beratung 3/17, 138-143

Yeryomenko, N. (2012): Does the depth of client experiencing predict good psychotherapy outcomes? A meta-analysis of treatment outcomes. A Thesis submitted to the Faculty of Graduate Studies through Department of Psychology at the University of Windsor. Electronic Theses and Dissertations 4847. Online unter: https://scholar.uwindsor.ca/etd/4847 (07.01.2020)

Zajonc, R. (1980). Social psychology: An experimental approach. Brooks: Cole

Zajonc, R. \& Hazel, M. (1982): Affective and Cognitive Factors in Preferences. Journal of Consumer Research, 9/2, 123-131. Online unter: https://www.jstor.org/stable/2489121 (21.03.2020) 


\section{Anhang}

\subsection{Verzeichnis der digitalen Anlagen}

Die Original-Audiographien und die Legenden zu den Transkriptionen werden hier zwar aufgeführt, aus Datenschutzgründen aber nicht veröffentlicht.

Die Nummerierung der Anlagen erfolgte chronologisch. Der Übersicht halber sind sie hier thematisch geordnet.

Die Anlagen sind online auf den Seiten der Kassel University Press abrufbar: https://kup.unikassel.de bzw. https://doi.org/doi:10.17170/kobra-202011182205.

\subsubsection{Anlagen zur Task-Analyse}

Anlage 1: Experiencing-Skala: Ratinginstruktionen und Stufenbeispiele:

DissUS_ExperiencingSkala.pdf

Anlage 2: Forschungstagebuch: DissUS_Forschungstagebuch.pdf

Anlage 5: Gesamtstichprobe - Übersicht: DissUS_GesamtstichprobeUebersicht.pdf

Anlage 5: Die Coachingprozesse im Detail: DissUS DieCoachingprozesseimDetail.pdf

Anlage 22: Relevante Stichprobe: DissUS_RelevanteStichprobe.pdf

Anlage 53: Sample Bildung: DissUS_SampleBildung.pdf

Anlage 54: Task Analyse Prozess 2017_1805_01\#1:

DissUS_TA_2016_1119a_01\#1.pdf

Anlage 55: Task Analyse Prozess 2016_1119a_01\#8:

DissUS_TA_2016_1119a_01\#8.pdf

Anlage 56: Task Analyse Prozess 2017_2119_03\#3: DissUS_TA_2017_2119_03\#3.pdf

Anlage 57: Task Analyse Prozess 2015_2007_02\#1: DissUS_TA_2015_2007_02\#1.pdf

Anlage 58: Task Analyse Prozess 2017_0118_01\#2: DissUS_TA_2017_0118_01\#2.pdf

Anlage 59: Task Analyse Prozess 2016_1119a_01\#1:

DissUS_TA_2016_1119a_01\#1.pdf

Anlage 60: Empirische Analyse, Higher-Level-Codierungen:

DissUS_HigherLevelCodierungen.pdf

Anlage 61: Vergleich der empirischen Modelle:

DissUS_VergleichderempirischenModelle.pdf

Anlage 62: Re-Rating und Berechnung der Re-Rating-Reliabilität:

DissUS_ReRatingReliability.pdf 


\subsubsection{Kasseler Coachingstudie}

Anlage 3: Projektflyer zur Kasseler Coachingstudie: ProjektflyerKCS.pdf

Anlage 4: Kasseler Coachingstudie, Fragebogendaten:

DissUS_KasselerCoachingstudieFragebogendaten.xlsx

Anlage 6: Audiographien zu Prozess 2015_011_01: Audios_2015_0111_01.zip

Anlage 7: Audiographien zu Prozess 2015_16_0111_02: Audios_2015_16_0111_02.zip

Anlage 8: Audiographien zu Prozess 2015_2007_01: Audios_2015_2007_01.zip

Anlage 9: Audiographien zu Prozess 2015_2007_02: Audios_2015_2007_02.zip

Anlage 10: Audiographien zu Prozess 2016_0319_01: Audios_2016_0319_01.zip

Anlage 11: Audiographien zu Prozess 2016_1119a_01: Audios_2016_1119a_01.zip

Anlage 12: Audiographien zu Prozess 2017_0118_01: Audios_2017_0118_01.zip

Anlage 13: Audiographien zu Prozess 2017_0118_02: Audios_2017_0118_02.zip

Anlage 14: Audiographien zu Prozess 2017_0118_03: Audios_2017_0118_03.zip

Anlage 15: Audiographien zu Prozess 2017_0118_04: Audios_2017_0118_04.zip

Anlage 16: Audiographien zu Prozess 2017_1805_01: Audios_2017_1805_01.zip

Anlage 17: Audiographien zu Prozess 2017_1805_02: Audios_2017_1805_02.zip

Anlage 18: Audiographien zu Prozess 2017_1805_03: Audios_2017_1805_03.zip

Anlage 19: Audiographien zu Prozess 2017_2119_01: Audios_2017_2119_01.zip

Anlage 20: Audiographien zu Prozess 2017_2119_02: Audios_2017_2119_02.zip

Anlage 21: Audiographien zu Prozess 2017_2119_03: Audios_2017_2119_03.zip

\subsubsection{Transkriptionen}

Anlage 23: Leitfaden zur Erstellung von Transkripten:

DissUS_KCSTranskriptionsleitfaden.pdf

Anlage 24: Legende zu Prozess 2015_0111_01: DissUS_Legende_2015_0111_01.pdf

Anlage 25: Legende zu Prozess 2015_0111_02: DissUS_Legende_2015_0111_02.pdf

Anlage 26: Legende zu Prozess 2015_2007_01: DissUS_Legende_2015_2007_01.pdf

Anlage 27: Legende zu Prozess 2015_2007_02: DissUS_Legende_2015_2007_02.pdf

Anlage 28: Legende zu Prozess 2016_1119a_01: DissUS_Legende_2016_1119a_01.pdf

Anlage 29: Legende zu Prozess 2017_0118_01: DissUS_Legende_2017_0118_01.pdf

Anlage 30: Legende zu Prozess 2017_1805_01: DissUS_Legende_2017_1805_01.pdf

Anlage 31: Legende zu Prozess 2017_2119_01: DissUS_Legende_2017_2119_01.pdf

Anlage 32: Legende zu Prozess 2017_2119_03: DissUS_Legende_2017_2119_03.pdf

Anlage 33: Transkript zu Coachingsitzung 2015_0111_01\#2a.:

DissUS_Transkript_2015_0111_01\#2a.pdf

Anlage 34: Transkript zu Coachingsitzung 2015_0111_01\#5:

DissUS_Transkript_2015_0111_01\#5.pdf 
Anlage 35: Transkript zu Coachingsitzung 2015_0111_02\#1:

DissUS_Transkript_2015_0111_02\#1.pdf

Anlage 36: Transkript zu Coachingsitzung 2015_0111_02\#1:

DissUS_Transkript_2015_0111_02\#1.pdf

Anlage 37: Transkript zu Coachingsitzung 2015_2007_02\#1:

DissUS_Transkript_2015_2007_02\#1.pdf

Anlage 38: Transkript zu Coachingsitzung 2016_1119a_01\#1:

DissUS_Transkript_2016_1119a_01\#1.pdf

Anlage 39: Transkript zu Coachingsitzung 2016_1119a_01\#4:

DissUS_Transkript_2016_1119a_01\#4.pdf

Anlage 40: Transkript zu Coachingsitzung 2016_1119a_01\#7:

DissUS_Transkript_2016_1119a_01\#7.pdf

Anlage 41: Transkript zu Coachingsitzung 2016_1119a_01\#8:

DissUS_Transkript_2016_1119a_01\#8.pdf

Anlage 42: Transkript zu Coachingsitzung 2017_0118_01\#1:

DissUS_Transkript_2017_0118_01\#1.pdf

Anlage 43: Transkript zu Coachingsitzung 2017_0118_01\#2:

DissUS_Transkript_2017_0118_01\#2.pdf

Anlage 44: Transkript zu Coachingsitzung 2017_1805_01\#1:

DissUS_Transkript_2017_1805_01\#1.pdf

Anlage 45: Transkript zu Coachingsitzung 2017_1805_01\#2:

DissUS_Transkript_2017_1805_01\#2.pdf

Anlage 46: Transkript zu Coachingsitzung 2017_1805_01\#3:

DissUS_Transkript_2017_1805_01\#3.pdf

Anlage 47: Transkript zu Coachingsitzung 2017_1805_01\#4:

DissUS_Transkript_2017_1805_01\#4.pdf

Anlage 48: Transkript zu Coachingsitzung 2017_1805_01\#5:

DissUS_Transkript_2017_1805_01\#5.pdf

Anlage 49: Transkript zu Coachingsitzung 2017_2119_01\#1:

DissUS_Transkript_2017_2119_01\#1.pdf

Anlage 50: Transkript zu Coachingsitzung 2017_2119_01\#2:

DissUS_Transkript_2017_2119_01\#2.pdf

Anlage 51: Transkript zu Coachingsitzung 2017_2119_01\#3:

DissUS_Transkript_2017_2119_01\#3.pdf

Anlage 52: Transkript zu Coachingsitzung 2017_2119_03\#3:

DissUS_Transkript_2017_2119_03\#3.pdf 


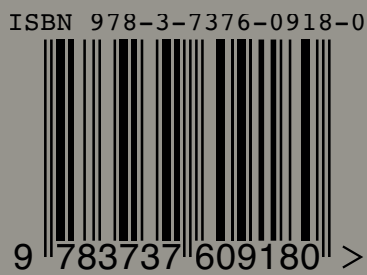

Im experienziellen Veränderungskonzept des Focusing spielt der Felt Sense eine besondere Rolle. Für das Coaching scheint das insofern relevant, als dass sich dort vor dem Hintergrund neurowissenschaftlicher Erkenntnisse zunehmend die Frage nach dem Einbezug affektiver und körperbezogener Aspekte stellt. Im Rahmen einer taskanalytischen explorativen Studie entwickelt der Autor ein auf Coaching bezogenes Modell intraindividueller Veränderung, an dessen Ausgangspunkt TrilemaSituationen im Sinn einer Inkongruenz oder Spannung zwischen Situationsbezug und kontradiktorischen Anteilen der affektiv-kognitiven Wahrnehmung stehen. Darauf aufbauend kann der Felt Sense bzw. das inm zugrunde liegende Experiencing als Ressource für einen themenbezogenen Klärungs- und Lösungsprozess genutzt werden. 